Espaços de funções e a propriedade de Lindelöf no produto

Renan Maneli Mezabarba 
SERVIÇO DE PÓS-GRADUAÇÃO DO ICMC-USP

Data de Depósito:

Assinatura:

\title{
Espaços de funções e a propriedade de Lindelöf no produto
}

\author{
Renan Maneli Mezabarba
}

Orientador: Prof. Dr. Leandro Fiorini Aurichi

Dissertação apresentada ao Instituto de Ciências Matemáticas e de Computação - ICMC-USP, como parte dos requisitos para obtenção do título de Mestre em Ciências - Matemática . VERSÃO REVISADA 
Ficha catalográfica elaborada pela Biblioteca Prof. Achille Bassi e Seção Técnica de Informática, ICMC/USP, com os dados fornecidos pelo(a) autor(a)

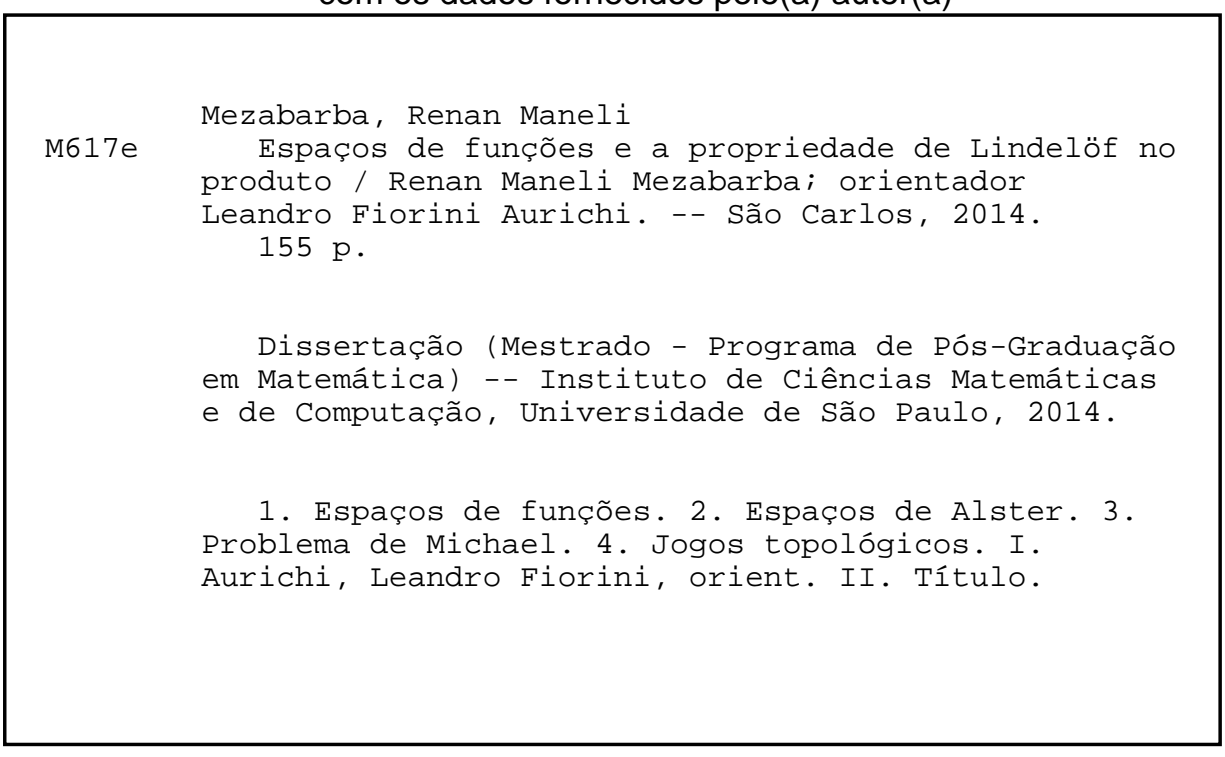


Para a minha família,

a coleção finita mais importante que conheço. 



\section{Agradecimentos}

Tentei ordenar os agradecimentos cronologicamente, na vã tentativa de evitar esquecimentos, bem como ser sucinto nas justificativas, para que tudo isso coubesse numa só página, o que se mostrou inútil uma vez que precisei aumentar o espaçamento entre as linhas...

Sendo assim, agradeço primeiramente a minha família - Sr. Antonio, D. Nadir e Tia Nilda :p -, não só por ela ser uma condição necessária para a minha existência (mas não unicidade, como bem provaram as disciplinas do mestrado), mas principalmente por todo o apoio, torcida e paciência que depositaram em mim. Em particular, agradeço à "PAIpesp" pelo auxílio financeiro nos primeiros meses do mestrado, como aluno especial.

Aos professores que conheci durante a graduação, que de uma forma ou de outra sempre me incentivaram a prosseguir na Matemática Pura; em particular, agradeço à professora Irene Craveiro e ao professor Elias Galante, bem como aos professores Marcelo Passos e Rodrigo Rock Days, pelo apoio e inspiração. Minha gratidão também pertence tanto aos meus amigos da graduação pela torcida (os quais não listo, por serem muitos), quanto às mariposas pelo "incentivo" indireto :P

Aos amigos que fiz aqui no ICMC, sem os quais eu certamente já teria voltado para casa. Destaco alguns: Ballbino e Lucas Galvão (cujos estudos nas salinhas da biblioteca renderam boas risadas e demonstrações), Jean Berni, (Aero, Pa, Peru)Lito e Érik; aos irmãos topológicos, Dione (e seus cadernos!), Guilherme, Henry, Hugo, Luan (e suas perguntas!) e ao Sandro também (embora este tenha debandado para a Filosofia). Aos amigos que fiz fora do ICMC, o que compreende essencialmente toda a galera da "República" Safu ao longo destes trinta meses, dos quais (por brevidade) cito apenas a D. Angela, uma segunda mãe para mim aqui 
em São Carlos. Agradeço ainda ao Thales, pelos açaís matemáticos - amigo desde a época da graduação -, e também à Lilian, pelas conversas, recomendações de séries e por me passar o modelo no qual eu digitei esta dissertação pela maior parte do tempo (no final das contas, usei uma fusão dos modelos da Lilian, do Dione e do Jean).

Agradeço às pessoas que constituem o ICMC, em particular: ao professor Sérgio Monari, pela ajuda durante a matrícula como aluno especial; aos professores Eduardo Tengan e Paulo Dattori, pelas excelentes aulas e pelo bom humor; aos diversos funcionários que integram a Pós-Graduação do ICMC, em especial ao Alexandre Reis, que muito pacientemente me ajudou com as burocracias da pré-Defesa.

Ao professor Angelo Bella, que melhorou consideravelmente um dos resultados obtidos neste trabalho (Teorema 2.3.22, grazie mille! Também agradeço à banca examinadora, pela leitura deste trabalho e pelas sugestões feitas na Defesa.

Com ênfase extra (e consideravelmente fora da ordem cronológica), registro aqui meus agradecimentos ao professor Leandro Aurichi, pela orientação, confiança e pelas excelentes sugestões de leitura, sem as quais nenhuma linha deste trabalho seria possível.

A todos que me ajudam a perseguir esta Torre Negra: muito obrigado por vocês existirem!

Por fim, agradeço o apoio financeiro da Capes durante o mestrado. 
"The man in black fled across the desert, and the gunslinger followed." Stephen King, The Dark Tower. 



\section{Resumo}

Neste trabalho, alguns espaços de funções que surgem naturalmente no contexto da topologia geral são estudados. Por meio da noção de bornologias, problemas de $C_{p}$-teoria e $C_{k}$-teoria são analisados simultaneamente, como a caracterização de certas funções cardinais no espaço das funções contínuas e propriedades relativas a jogos seletivos. A compactificação de StoneČech também é estudada, onde a existência de $P$-pontos no resíduo dos naturais é considerada sob a Hipótese do Contínuo. Com a adição de certas hipóteses sobre pequenos cardinais, alguns resultados obtidos ao longo do texto são utilizados em problemas relacionados com espaços de Michael e espaços de Alster.

Palavras-chave: espaços de funções, $C_{p}$-teoria, $C_{k}$-teoria, bornologias, funções cardinais, jogos topológicos, pequenos cardinais, espaços de Alster, Problema de Michael. 



\section{Abstract}

In this work, some function spaces that naturally arise in the general topology context are studied. By the notion of bornologies, $C_{p}$-theory and $C_{k}$-theory problems are simultaneously analysed, as the characterization of some cardinal functions in the space of continuous real functions and properties related to selective games. The Stone-Čech compactification is also studied, and the existence of $P$-points in the remainder of the set of the natural numbers is considered under the Continuum Hypothesis. With the addition of some hypotheses about small cardinals, some results obtained through the text are used in problems related to Michael spaces and Alster spaces.

Keywords: function spaces, $C_{p}$-theory, $C_{k}$-theory, bornologies, cardinal functions, topological games, small cardinals, Alster spaces, Michael's Problem. 



\section{Sumário}

\begin{tabular}{ll}
\hline Introdução & 15
\end{tabular}

\begin{tabular}{lcc}
\hline & Preliminares & 17
\end{tabular}

1.1 Teoria dos Conjuntos . . . . . . . . . . . . . . . . 17

1.2 Topologia . . . . . . . . . . . . . . . . . . . 21

1.3 Uniformidades e espaços uniformes $\ldots \ldots \ldots$. . . . . . . . . . 28

1.4 Jogos topológicos seletivos . . . . . . . . . . . . . . . . . 34

2 Tópicos de $C_{p}$-Teoria e $C_{k}$-Teoria

2.1 Bornologias . . . . . . . . . . . . . . . . . 41

$2.1 .1 \quad$ Bornologias e propriedades de recobrimento . . . . . . . . . . 44

2.1 .2 Topologia da convergência uniforme numa bornologia . . . . . . . . . 49

$2.2 \quad$ Propriedades de enumerabilidade em $C_{\mathcal{B}}(X) \ldots \ldots \ldots \ldots \ldots$

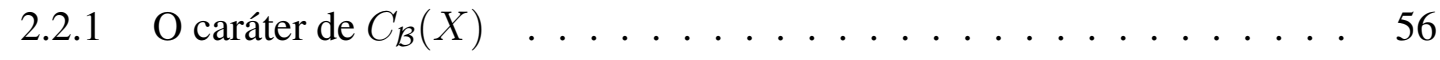

2.2 .2 O peso de $C_{\mathcal{B}}(X) \ldots \ldots \ldots \ldots \ldots \ldots$

2.2 .3 Densidade e celularidade de $C_{\mathcal{B}}(X) \ldots \ldots \ldots \ldots$

$2.3 \quad$ Variações em tightness de $C_{\mathcal{B}}(X) \ldots \ldots \ldots \ldots \ldots \ldots$

$2.3 .1 \quad \mathrm{O}$ tightness de $C_{\mathcal{B}}(X)$ e a propriedade de Fréchet $\ldots . . \ldots 73$

$2.3 .2 \quad$ Variações seletivas de tightness em $C_{\mathcal{B}}(X) \ldots \ldots$. . . . . . . 78

2.3 .3 O tightness de $C_{\mathcal{B}}(X)$ e a operação produto . . . . . . . . . . . . . 89

2.4 Apêndice do Capítulo . . . . . . . . . . . . . . . . . . . 93

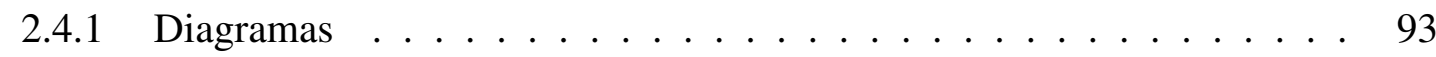


2.4 .2 Convergência uniforme forte - o caso métrico . . . . . . . . . 95

2.4 .3 Demonstração do Teorema 2.3 .20 (de Arhangel'skii) . . . . . . . . . . 99

\begin{tabular}{|lll}
3 & A Compactificação de Stone-Čech & 107
\end{tabular}

3.1 Existência da compactificação de Stone-Čech . . . . . . . . . . . . . . 107

$3.2 \quad P$-pontos em $\beta \omega \backslash \omega$ na presença de $\mathrm{CH} \ldots \ldots \ldots \ldots$. . . . . . . . 121

4 Aplicações: Espaços Produtivamente de Lindelöf 129

$4.1 \quad$ Espaços de Alster I: Aplicações de $C_{\mathcal{B}}$-teoria $\ldots \ldots \ldots \ldots$

4.2 Espaços de Alster II: Aplicações da compactificação de Stone- Čech . . . . . . 133

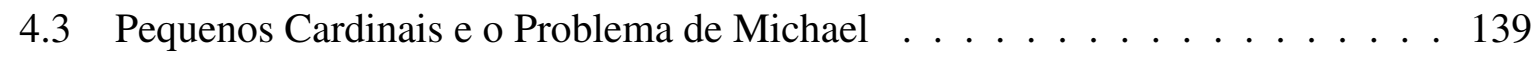

\begin{tabular}{ll}
\hline Referências Bibliográficas & 147 \\
\hline
\end{tabular}

Índice Remissivo 152 


\section{Introdução}

Dados dois espaços topológicos, é natural nos perguntarmos como as noções topológicas dos espaços dados influenciam a noção de proximidade entre duas funções consideradas entre esses espaços. De modo mais técnico: se $X$ e $Y$ são espaços topológicos, existe alguma topologia natural no espaço das funções de $X$ em $Y$ que esteja relacionada com as topologias de $X$ e $Y$ ? A resposta varia de acordo com o contexto no qual nos situamos mas, de modo geral, ela é afirmativa e não é necessariamente única.

Neste trabalho, apresentamos algumas das possíveis respostas, e também investigamos certos casos de espaços de funções munidos de tais topologias. Inicialmente, era objetivo estudarmos três situações de forma independente:

1. a topologia da convergência uniforme forte numa bornologia ( $c f$. Seção 2.4.2);

2. a compactificação de Stone-Čech de um espaço topológico, definida como um espaço de funções ( $c f$. Capítulo 3);

3. alguns pequenos cardinais, invariantes topológicos do espaço das funções de $\omega$ em $\omega$ ( $c f$. Seção 4.3.

A topologia referida no item (1) acima (e definida em [6]) se dá para espaços de funções entre espaços métricos, e nossa proposta era estender os resultados de [6] para contextos mais gerais. Por outro lado, os objetivos para os itens (2) e (3) acima consistiam em estudar seus fundamentos e aplicações, em particular, no que tange aos espaços produtivamente de Lindelöf (cf. Capítulo 4), como feito em [1]. Contudo, com o desenrolar do projeto, aplicações de (1) para o mesmo problema também surgiram e, desse modo, todo o trabalho "convergiu" para 
aplicações em espaços produtivamente de Lindelöf. Por tal motivo, estruturamos o texto da forma como descrevemos a seguir.

No primeiro capítulo, apresentamos os resultados básicos que serão utilizados ao longo do texto. Fazemos uma breve revisão sobre Teoria dos Conjuntos e Topologia, bem como sobre espaços uniformes. Em seguida, apresentamos uma breve sistematização sobre jogos seletivos e alguns exemplos de suas aplicações em topologia, na forma de jogos topológicos seletivos.

O segundo capítulo consiste dos desdobramentos do item (1) descrito acima, o que ocupa a maior parte deste trabalho. Uma possível generalização da topologia definida em [6] para espaços de Tychonoff se reduz as ditas topologias de convergência uniforme (já estudadas, por exemplo, por McCoy e Ntantu [31]), que são definidas em termos de uma estrutura no espaço base, as bornologias, o que fazemos na primeira seção deste capítulo. Nas duas seções seguintes, caracterizamos algumas funções cardinais do espaço das funções reais contínuas definidas num espaço de Tychonoff, de maneira semelhante a apresentada em [31], e analisamos algumas variações seletivas da função cardinal tightness, onde generalizamos alguns resultados já conhecidos. Na última seção, comentamos brevemente a topologia definida em [6], além de apresentarmos uma demonstração relativamente extensa de um resultado de Arhangel'skii utilizado na seção anterior.

No Capítulo 3, voltamo-nos para a compactificação de Stone-Čech. A primeira seção se foca nas questões de existência e unicidade (a menos de homeomorfismo) da compactificação de Stone-Čech de um espaço de Tychonoff, onde também examinamos algumas propriedades básicas e exemplos. Na seção seguinte, estreitamos nossa análise para a compactificação de Stone-Čech dos naturais, mais precisamente no que tange à existência dos chamados P-pontos, onde seguimos essencialmente a abordagem de Rudin em [37].

No quarto capítulo apresentamos algumas aplicações concernentes aos espaços produtivamente de Lindelöf. Na primeira e segunda seções, utilizamos respectivamente resultados do segundo e terceiro capítulos para obtermos informações sobre tal classe de espaços. A última seção se restringe a alguns pequenos cardinais $(\mathfrak{b}, \mathfrak{d}$ e $\operatorname{cov}(\mathcal{M}))$ e às suas aplicações no chamado Problema de Michael, o qual essencialmente consiste em saber se o espaço dos números irracionais com a topologia usual é um espaço produtivamente de Lindelöf. 


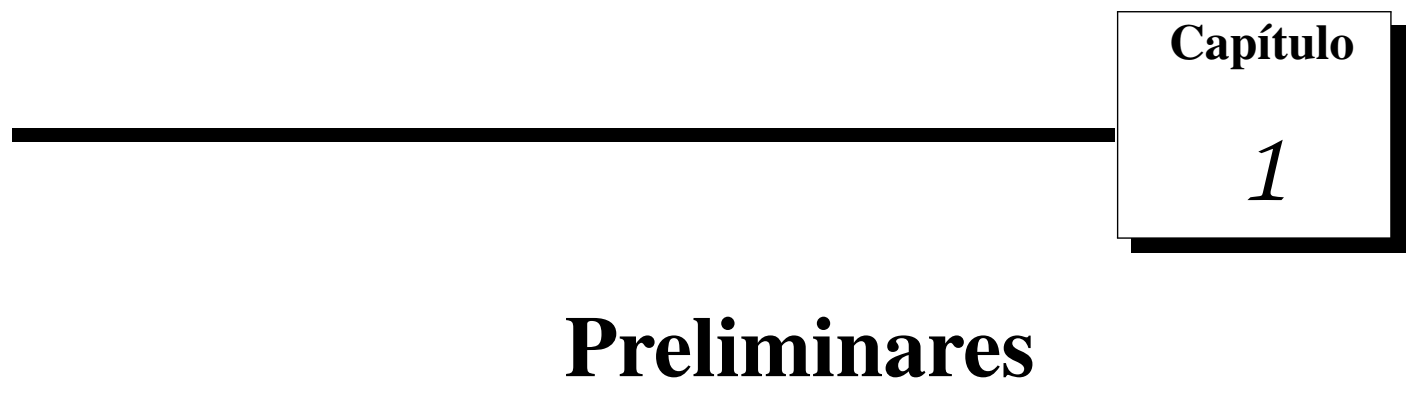

O objetivo deste capítulo é fornecer o ferramental básico para o desenvolvimento dos capítulos subsequentes. Definiremos os principais conceitos de Teoria dos Conjuntos e Topologia que serão utilizados, além de fixarmos as notações e relembrarmos alguns resultados importantes. Em cada seção, indicaremos as referências nas quais as demonstrações omitidas podem ser encontradas, bem como tópicos relacionados que possam interessar ao leitor.

\subsection{Teoria dos Conjuntos}

Nesta seção, fazemos uma introdução breve sobre Teoria dos Conjuntos, principalmente com o intuito de fixar as notações. Os livros de Jech [25] e Kunen [29] cobrem profundamente todos os tópicos que abordaremos sobre Teoria dos Conjuntos, embora um texto introdutório, como o de Hrbacek e Jech [23], seja suficiente para preencher a maioria (senão todas) das lacunas desta seção. Em todo o decorrer deste trabalho, assumimos o sistema axiomático Zermelo-Fraenkel acrescido do Axioma da Escolha (ZFC).

As operações usuais entre conjuntos (interseção, reunião, diferença, potência e produto) serão supostos conhecidos. A diferença entre dois conjuntos $X$ e $Y$ será denotada por $X \backslash Y$, e a coleção de todos os subconjuntos de $X$ será indicada por $\wp(X)$. Uma relação (binária) é um conjunto de pares ordenados (e, por conseguinte, relações de ordem, de equivalência e funções também). Em particular, se $X$ é um conjunto, então qualquer elemento de $\wp(X \times X)$ 
é uma relação e, desse modo, ficam bem definidas as operações de composição e inversão em $\wp(X \times X)$, mais precisamente: se $A, B \subseteq X \times X$, então $A^{-1}=\{(y, x):(x, y) \in A\}$ e $A \circ B=$ $\{(x, y): \exists z((x, z) \in B \wedge(z, y) \in A)\}$ também são subconjuntos de $X \times X$ (cf. Seção 1.3). Assumimos conhecidas as definições e notações usuais referentes a relações de equivalência, ordens parciais e funções, bem como resultados básicos envolvendo tais tópicos. Em particular, relembramos o leitor de que uma relação de ordem $\leq$ sobre $X$ é uma boa ordenação (ou boa ordem) se todo subconjunto não vazio de $X$ possui um elemento mínimo com respeito a $\leq$.

Dados conjuntos $X$ e $Y$, denotaremos por $Y^{X}$ o conjunto de todas as funções $f: X \rightarrow Y$, isto é, das funções com domínio $X$ e contradomínio $Y$. Se $A \subseteq X, f[A]:=\{f(x): x \in A\}$ é a imagem direta de $A$ por $f$ e, para $B \subseteq Y, f^{-1}[B]:=\{x \in X: f(x) \in B\}$ denota a préimagem (ou imagem inversa) de $B$ por $f$; também indicamos por $f \uparrow A$ a função de domínio $A$ e contradomínio $Y$ que associa a cada $a \in A$ o elemento $f(a) \in Y$, isto é, $f\lceil A$ é a restrição de $f$ ao subconjunto $A$. Quando $Y=X$, denotamos por $i d_{X}:=\Delta(X):=\{(x, x): x \in X\}$ a função identidade de $X$ - ou simplesmente por $i d$ e $\Delta$, quando o conjunto $X$ estiver claro pelo contexto. Dada uma família $\mathcal{F}=\left\{X_{i}: i \in I\right\}$ de conjuntos, a coleção de todas as funções $f: I \rightarrow \bigcup_{i \in I} X_{i}$ tais que $f(i) \in X_{i}$, o produto da família $\mathcal{F}$, será denotado por $\prod_{i \in I} X_{i}$. A reunião disjunta de $\mathcal{F}$, indicada por $\bigsqcup_{i \in I} X_{i}$, é por definição ${ }^{1}$ o conjunto $\bigcup_{i \in I}\left(X_{i} \times\{i\}\right)$.

Dizemos que $X$ é um conjunto transitivo se todo elemento de $X$ é também subconjunto de $X ; X$ é um número ordinal (ou simplesmente um ordinal) se $X$ for transitivo e $\left(X, \in_{X}\right)$ for uma boa ordem, onde $\epsilon_{X}=\{(x, y) \in X \times X: x \in y\}$, o que faz com que todos os elementos de um ordinal sejam ordinais. Geralmente, denotamos ordinais por letras gregas minúsculas $(\alpha, \beta, \gamma$, etc.) e, para dois ordinais $\alpha$ e $\beta$, escrevemos $\alpha<\beta$ para indicar que $\alpha \in \beta-$ em particular, vale a tricotomia para todos ${ }^{2}$ os ordinais, isto é, dados ordinais $\alpha$ e $\beta$, necessariamente um (e apenas um) dos seguintes casos ocorre: $\alpha<\beta$ ou $\beta<\alpha$ ou $\alpha=\beta$. Assim, para qualquer ordinal $\alpha$ vale a igualdade $\alpha=\{\beta: \beta<\alpha\}$. Todo conjunto $A$ de ordinais admite um mínimo e um supremo, a saber, $\min A=\bigcap A$ e $\sup A=\bigcup A$ (a posteriori, o mesmo vale para conjuntos de cardinais). Além disso, se $(A, R)$ é uma boa ordem, então

\footnotetext{
${ }^{1} \mathrm{~A}$ menos que os elementos de $\mathcal{F}$ sejam dois a dois disjuntos, caso em que não fazemos distinção entre os operadores $\bigsqcup$ e $\bigcup$.

${ }^{2}$ Formalmente, em ZFC não existe o conjunto de todos os ordinais.
} 
existe um único ordinal $\alpha$ tal que $(A, R)$ e $\left(\alpha, \in_{\alpha}\right)$ são isomorfos (no sentido de ordem); tal ordinal é chamado de tipo de ordem de $(A, R)$ (order type).

Dados um ordinal $\alpha$ e uma função $f: \beta \rightarrow \alpha$, diremos que $f$ é uma função cofinal em $\alpha$ se para todo $\gamma<\alpha$ existe $\delta<\beta$ tal que $\gamma \leq f(\delta)$; define-se então a cofinalidade de $\alpha$ como sendo o menor ordinal $\beta$ para o qual existe uma função $f: \beta \rightarrow \alpha$ cofinal em $\alpha$, denotado por $\operatorname{cof}(\alpha)$ - claramente $\operatorname{cof}(\alpha) \leq \alpha$. Definimos o sucessor de um ordinal $\alpha$ como sendo $\alpha+1:=\alpha \cup\{\alpha\}$; $\alpha$ é um ordinal sucessor se existir $\beta$ tal que $\alpha=\beta+1$, caso contrário, $\alpha$ é um ordinal limite - por exemplo, $0:=\emptyset$ é um ordinal limite por vacuidade, e $1:=0+1=\{0\}$ é um ordinal sucessor.

Um ordinal $\alpha$ é um número natural se para todo $\beta<\alpha, \beta$ for sucessor ou $\beta=0$. Usualmente simbolizamos números naturais por letras minúsculas do nosso alfabeto $(m, n, p$, etc.). Denotamos por $\omega\left(\text { ou } \aleph_{0} \text { ) a coleção de todos os números naturai }\right\}^{3}$ que é o menor ordinal limite diferente de 0 . Chamamos uma função $f: \omega \rightarrow X$ de sequência em $X$, e denotamos $f=\left(x_{n}\right)_{n \in \omega}$, onde $f(n)=x_{n}$ - em geral, qualquer função da forma $f: \alpha \rightarrow X$ será chamada de sequência em $X$, e escreveremos $f=\left(x_{\beta}\right)_{\beta<\alpha}$, onde $f(\beta)=x_{\beta}$.

Para conjuntos $X$ e $Y$ quaisquer, dizemos que $X$ e $Y$ são equipotentes se existir uma função bijetora $f: X \rightarrow Y$, o que simbolizamos por $|X|=|Y|$. Um conjunto $X$ é finito (infinito enumerável) caso $X$ seja equipotente a um número natural (ou equipotente a $\omega$, respectivamente), do contrário $X$ é não-enumerável. Um ordinal $\alpha$ é um número cardinal se não existir $\beta<\alpha$ tal que $|\beta|=|\alpha|$ - geralmente denotamos cardinais por letras gregas minúsculas ( $\kappa, \lambda$, $\mu$, etc.). Como todo conjunto bem ordenado é isomorfo a um único ordinal, para um conjunto bem ordenável $X$ fica bem definido o número cardinal $|X|:=\min \{\alpha:|\alpha|=|X|\}$, a cardinalidade de $X$. Logo, em ZFC, todo conjunto tem um número cardinal, haja vista que todo conjunto é bem ordenável pelo Axioma da Escolha.

Assumimos conhecidos os resultados básicos de aritmética cardinal e ordinal. Em particular, frisamos o Teorema de Cantor, que estabelece a desigualdade $|X|<|\wp(X)|$, da qual segue $\omega<|\wp(\omega)|$ e, consequentemente, $\omega_{1} \leq|\wp(\omega)|:=\mathfrak{c}$, onde $\omega_{1}$ (ou $\aleph_{1}$ ) é o menor ordinal (cardinal) não-enumerável e c é a cardinalidade do contínuo. A Hipótese do Contínuo é a asserção $\aleph_{1}=\mathfrak{c}$.

\footnotetext{
${ }^{3}$ Como tal conjunto satisfaz os Axiomas de Peano, o conjunto $\omega$ é para todos os efeitos (lê-se a menos de isomorfismo) o conjunto dos números naturais usualmente denotado por $\mathbb{N}$.
} 
Fixados um conjunto $X$ e um cardinal $\kappa$, definimos:

(a) $[X]^{<\kappa}:=\{Y \subseteq X:|Y|<\kappa\}$;

(b) $[X]^{\leq \kappa}:=\{Y \subseteq X:|Y| \leq \kappa\}$;

(c) $[X]^{\kappa}:=\{Y \subseteq X:|Y|=\kappa\}$;

(d) $X^{<\kappa}:=\bigcup_{\lambda<\kappa} X^{\lambda}$ e $X^{\leq \kappa}:=X^{<\kappa} \cup X^{\kappa}$.

Em particular, a igualdade $\omega=\left|[\omega]^{<\omega}\right|$ será de grande utilidade nos próximos capítulos. Essencialmente, para verificá-la basta notar que $[\omega]^{<\omega}=\bigcup_{n<\omega}[\omega]^{n}$, com $\left|[\omega]^{n}\right|=\omega$, e usar o fato de que a reunião enumerável de conjuntos enumeráveis é enumerável. Mais geralmente, $\left|[A]^{<\omega}\right|=|A|$ para qualquer conjunto infinito $A$. 


\subsection{Topologia}

Apresentaremos as definições e os resultados básicos de Topologia Geral que serão adotados ao longo do texto. De modo geral, utilizamos os termos e notações do livro de Engelking [15], embora não façamos as mesmas suposições acerca de axiomas de separação para certas definições. Também seguimos os textos de Tkachuk [47] e Willard [50], de modo que em tais referências as demonstrações omitidas nesta seção podem ser encontradas. Além disso, assumiremos que o leitor já tenha feito um curso de Topologia Geral e de Espaços Métricos, estando assim familiarizado com a terminologia usual da teoria (abertos, fechados, fecho ou fechamento, interior, funções contínuas, etc.).

Fixemos um espaço topológico $(X, \tau)$, um ponto $x \in X$ e um subconjunto $A \subseteq X$. Quando a topologia $\tau$ estiver clara pelo contexto, vamos nos referir a $(X, \tau)$ simplesmente como o "espaço topológico $X$ " ou mesmo como o "espaço $X$ ". Dizemos que $A$ é uma vizinhança de $x$ se existir um aberto $V \subseteq X$ tal que $x \in V \subseteq A$, sem que $A$ seja necessariamente aberto. Denotamos o fecho (closure) de $A$ por $\bar{A}$ e o interior de $A$ por $i n t(A)$.

Um conjunto parcialmente ordenado $(L, \leq)$ é dirigido se para quaisquer $x, y \in L$ existir $w \in L$ tal que $x \leq w$ e $y \leq w$. Se $(L, \leq)$ é dirigido, uma função $f: L \rightarrow X$ é chamada de net em $X$, e usualmente denotamos $f=\left(x_{l}\right)_{l \in L}$, onde $f(l)=x_{l}$. Em particular, $\omega$ é um conjunto dirigido (pela ordem usual dos naturais), e uma net $\left(x_{n}\right)_{n \in \omega}$ é uma sequência em $X$. Uma net $\left(x_{l}\right)_{l \in L}$ em $X$ converge para $x$ se, para toda vizinhança $A$ de $x$ existir $l_{A} \in L$ tal que $x_{l} \in A$ para todo $l \geq l_{A}$, o que indicamos por $x_{l} \rightarrow x$, ou $x_{l} \rightarrow_{\tau} x$ quando quisermos evidenciar a topologia de $X$.

Seja $(T, \leq)$ um conjunto totalmente ordenado com pelo menos dois pontos. Para quaisquer $x, y \in T$, definimos:

(a) $] x, y[:=\{a \in T: x<a<y\}$;

(b) $] x, y]:=] x, y[\cup\{y\}$;

(c) $[x, y[:=\{x\} \cup] x, y[$;

(d) $[x, y]:=[x, y[\cup\{y\}$. 
Para um elemento $t \in T$ qualquer, declaramos como aberto básico de $t$ qualquer conjunto da forma $] x, y[$ tal que $t \in] x, y\left[\right.$ caso $t$ não seja máximo nem mínimo de $T$, do contráriq ${ }^{4}$ seus abertos básicos são da forma $[t, y[$ (se $t$ for mínimo) ou da forma $] x, t]$ (caso $t$ seja máximo). É fácil ver que isto de fato define uma topologia em $T$, a chamada topologia da ordem, que é de Hausdorff (ver definição na página seguinte). Em particular, quando $T$ é um ordinal $\alpha$, a topologia da ordem em $\alpha$ é tal que $\beta \in \alpha$ é isolado se, e somente se, $\beta$ é sucessor (e, consequentemente, $\beta$ é ordinal limite se, e somente se, $\beta$ não é isolado). A menos de menção em contrário, ao considerarmos um ordinal como espaço topológico, assumiremos que sua topologia é a topologia da ordem.

Dada uma família $\left\{\left(X_{i}, \tau_{i}\right): i \in I\right\}$ de espaços topológicos e um subconjunto $V=\prod_{i \in I} V_{i}$, onde cada $V_{i} \subseteq X_{i}$, definimos o suporte de $V$ como sendo o conjunto $\operatorname{supp}(V):=\left\{i \in I: V_{i} \neq\right.$ $\left.X_{i}\right\}$. Ao longo deste trabalho, exceto quando expresso o contrário, consideraremos o produto $\prod_{i \in I} X_{i}$ munido da topologia produto, que é a topologia gerada como base pelos conjuntos da forma $V=\prod_{i \in I} V_{i}$ de suporte finito, onde cada $V_{i} \in \tau_{i}$. Quando os conjuntos $X_{i}$ são dois a dois disjuntos, denotamos por $\sum_{i \in I} X_{i}$ a soma dos espaços $X_{i}$, definido como o conjunto $\bigsqcup_{i \in I} X_{i}$, munido da topologia gerada como base pela família $\bigcup_{i \in I} \tau_{i}$.

Denotamos por $\mathbb{R}$ o conjunto dos números reais munido da topologia usual induzida por sua métrica; a menos de menção contrária, consideramos qualquer conjunto de números reais com a topologia de subespaço de $\mathbb{R}$. Também assumimos conhecidos conceitos basicos referentes à Medida de Lebesgue em $\mathbb{R}$. Os símbolos $\mathbb{Z}, \mathbb{Q}$ e $\mathbb{P}$ denotam, respectivamente, o conjunto dos números inteiros, dos números racionais e dos números irracionais.

Geralmente, hipóteses adicionais referentes à topologia do espaço devem ser feitas para obtermos resultados interessantes. Ao longo de nosso trabalho, definiremos e estudaremos várias propriedades topológicas. Assim, a fim de tornarmos a leitura dos próximos capítulos menos carregada com várias definições, listamos a seguir algumas condições básicas que ocasionalmente serão impostas posteriormente, bem como alguns resultados relativos a elas, os quais serão utilizados sem maior ênfase.

- Dizemos que $X$ é um espaço $\mathbf{T}_{0}$ se para quaisquer $x, y \in X$ distintos existir um aberto que contém exatamente um desses pontos.

\footnotetext{
${ }^{4}$ Um elemento mínimo (ou máximo) caso exista, é único.
} 
- Se $\{x\}$ é fechado para todo $x \in X$, dizemos que $X$ é um espaço $\mathbf{T}_{1}$. Note que se $X$ é um espaço $T_{1}$, então $X$ também é $T_{0}$.

- Um espaço $X$ é de Hausdorff (ou $\mathbf{T}_{2}$ ) se para todo par $x, y \in X$ de pontos distintos existirem abertos disjuntos $A, B \subseteq X$ tais que $x \in A$ e $y \in B$. Claramente, todo espaço de Hausdorff também é $T_{1}$.

- Dizemos que $X$ é um espaço $\mathbf{T}_{3}$ se para quaisquer $x \in X$ e $F \subseteq X$ fechado dados, com $x \notin F$, existirem abertos disjuntos $A, B \subseteq X$ tais que $x \in A$ e $F \subseteq B$. Um espaço é regular se for $T_{3}$ e $T_{1}$; note que espaços regulares são de Hausdorff.

- Um espaço $X$ é $\mathbf{T}_{3 \frac{1}{2}}$ se para quaisquer $x \in X$ e $F \subseteq X$ fechado com $x \notin F$ existir uma função contínua $f: X \rightarrow[0,1]$ tal que $f(x)=0$ e $f[F] \subseteq\{1\}$. Um espaço é dito de Tychonoff se for $T_{3 \frac{1}{2}}$ e $T_{1}$. Em particular, todo espaço de Tychonoff é regular.

- Se para quaisquer $F, G \subseteq X$ fechados disjuntos existirem abertos disjuntos $A, B \subseteq X$ satisfazendo $F \subseteq A$ e $G \subseteq B$, diremos que $X$ é um espaço $\mathbf{T}_{4}$. Um espaço topológico é normal se for $T_{4}$ e $T_{1}$. O Lema de Urysohn é a afirmação de que em espaços $T_{4}$ fechados disjuntos podem ser separados por funções contínuas, i.e., se $F, G \subseteq X$ são fechados disjuntos, então existe uma função contínua $f: X \rightarrow[0,1]$ tal que $f[F] \subseteq\{0\}$ e $f[G] \subseteq\{1\}$, o que acaba por também caracterizar espaços $T_{4}$. Desse modo, é evidente que espaços normais são de Tychonoff. Outra caracterização para espaços $T_{4}$ se dá pelo Teorema de Tietze: um espaço $X$ é $T_{4}$ se, e somente se, toda função contínua $f: A \rightarrow$ $[0,1]$ definida num fechado $A$ de $X$ admite uma extensão contínua com domínio $X$.

- Um espaço $X$ é compacto (respectivamente, de Lindelöf) se para toda coleção $\mathcal{U} \subseteq$ $\tau$ que recobre $X$, i.e., tal que $\bigcup \mathcal{U}=X$, existir $\mathcal{U}^{\prime} \in[\mathcal{U}]^{<\omega}$ (respectivamente, $\mathcal{U}^{\prime} \in$ $[\mathcal{U}]^{\leq \omega}$ ) tal que $X=\bigcup \mathcal{U}^{\prime}$. Um espaço é $\sigma$-compacto se for uma reunião enumerável de compactos. Diferente de [15], não incluímos as hipóteses de que espaços compactos sejam Hausdorff e que espaços de Lindelöf sejam regulares; se adicionadas, em ambos os casos elas garantem que tais espaços sejam normais. Claramente todo espaço compacto é $\sigma$-compacto e, por sua vez, espaços $\sigma$-compactos são de Lindelöf. O produto de espaços compactos é compacto (Teorema de Tychonoff), e o produto entre um espaço compacto 
e um espaço de Lindelöf é de Lindelöf ( $c f$. Capítulo 4), contudo existem espaços de Lindelöf cujo produto não é de Lindelöf.

- Espaços compactos "se comportam" como pontos 5 A sentença anterior não é um teorema formal, mas sim uma observação sobre o comportamento usual de espaços compactos. Por exemplo, se $X$ é de Hausdorff, $K, F \subseteq X$ são subespaços disjuntos e compactos de $X$, então existem abertos disjuntos $A$ e $B$ tais que $K \subseteq A$ e $F \subseteq B$; se $X$ é regular e somente $K$ é compacto, com $F$ fechado e $K \cap F=\emptyset$, então também podemos separar ambos os subconjuntos $K$ e $F$ por abertos disjuntos. A mesma "regra" se repete para espaços de Tychonoff: se num espaço de Tychonoff $X$ temos um compacto $K \subseteq X$ e um fechado $F \subseteq X$ tais que $K \cap F=\emptyset$, então existe uma função contínua $f: X \rightarrow[0,1]$ satisfazendo $f \uparrow K \equiv 0$ e $f \uparrow F \equiv 1$ - chamamos tal fato de Lema de Urysohn "Fraco".

- Outro importante fato sobre espaços compactos se dá no Teorema de Wallace: Seja $\left\{X_{s}\right\}_{s \in S}$ uma família de espaços topológicos não vazios; se $A_{s} \subseteq X_{s}$ é compacto para cada $s \in S$, então para todo $W$ aberto em $\prod_{s \in S} X_{s}$ tal que $\prod_{s \in S} A_{s} \subseteq W$, existem abertos $U_{s} \subseteq X_{s}$, com $U_{s} \neq X_{s}$ apenas para finitos elementos de $S$, satisfazendo

$$
\prod_{s \in S} A_{s} \subseteq \prod_{s \in S} U_{s} \subseteq W
$$

Demonstração. Vamos provar o teorema apenas para o caso em que $S=\{0,1\}$ - uma demonstração para o caso geral pode ser encontrada, por exemplo no Capítulo 3 de [15]. Primeiramente, fazemos a seguinte afirmação:

se $A$ é um subconjunto compacto não vazio de $X, y \in Y$ e $W \subseteq X \times Y$ é um aberto que contém $A \times\{y\}$, então existem $U \subseteq X, V \subseteq Y$ abertos tais que

$$
A \times\{y\} \subseteq U \times V \subseteq W .
$$

De fato, como $W$ é aberto, para cada $x \in A$ existem $U_{x} \subseteq X$ e $V_{x} \subseteq Y$ abertos tais que $(x, y) \in U_{x} \times V_{x} \subseteq W$. A compacidade de $A$ e as inclusões $A \times\{y\} \subseteq \bigcup_{x \in A} U_{x} \times V_{x} \subseteq W$ garantem a existência de uma família $\left\{x_{0}, \ldots, x_{k}\right\} \subseteq A$ tal que $A \times\{y\} \subseteq \bigcup_{i \leq k} U_{x_{i}} \times V_{x_{i}}$. Note que os abertos $U=\bigcup_{i \leq k} U_{i}$ e $V=\bigcap_{i \leq k} V_{i}$ têm a propriedade desejada.

\footnotetext{
${ }^{5} \mathrm{Ou}$ mais geralmente, compactos se comportam como conjuntos finitos.
} 
Agora, para provar o teorema, basta aplicar a afirmação anterior para cada compacto da forma $A_{0} \times\{y\}$, com $y \in A_{1}$, e obter os abertos $U_{0}$ e $U_{1}$ por meio da compacidade de $A_{1}$, de modo análogo ao que fizemos acima.

- Dizemos que $X$ satisfaz o primeiro axioma de enumerabilidade (ou que $X$ tem caráter enumerável, $c f$. Seção 2.2.1 se todo ponto de $X$ tem base local enumerável. O espaço $X$ satisfaz o segundo axioma de enumerabilidade (ou o peso de $X$ é enumerável, $c f$. Seção 2.2.2) se a topologia de $X$ admite uma base enumerável. Um espaço que contém um subespaço enumerável e denso é dito separável (ou de densidade enumerável, $c f$. Seção 2.2.3. Todo espaço métrico tem caráter enumerável. Além disso, um espaço topológico $X$ é regular e de peso enumerável se, e somente se, for metrizável e separável.

- Os termos caráter, peso e densidade destacados no item anterior são exemplos de funções cardinais. Uma função cardinal $\varphi$ é um invariante topológico (ou propriedade topológica), que a cada espaço topológico $(X, \tau)$ associa univocamente um número cardinal infinito $\varphi((X, \tau))$. Assim, apesar do nome, $\varphi$ não é uma função no sentido estrito do termo, pois $\varphi$ não define um conjunto em ZFC. Tais funções cardinais generalizam propriedades topológicas como as do item anterior para cardinais maiores do que $\aleph_{0}$.

Optamos por definir as funções cardinais ao longo do texto, conforme a ocasião torná-las necessárias. Em particular, como boa parte de nosso trabalho consiste no estudo de funções cardinais em espaços de funções, assumimos ao longo do texto que todos os espaços topológicos considerados são infinitos, a menos de menção contrária.

Fixemos espaços topológicos $(X, \tau)$ e $(Y, \rho)$. Lembremo-nos de que $Y^{X}=\prod_{x \in X} Y$, e assim podemos considerar o conjunto das funções de $X$ em $Y$ como um espaço topológico $\left(Y^{X}, \mathcal{T}_{p}\right)$, onde $\mathcal{T}_{p}$ é a topologia produto. Um ponto $f \in Y^{X}$ é uma função $f: X \rightarrow Y$ e, se $\left(f_{l}\right)_{l \in L}$ é uma net em $Y^{X}$, decorre diretamente da definição dos abertos básicos em $Y^{X}$ que $f_{l} \rightarrow_{\mathcal{T}_{p}} f$ se, e somente se, $f_{l}(x) \rightarrow_{\rho} f(x)$ para todo $x \in X$. Por isso, é comum chamar a topologia produto como a topologia da convergência pontual. Também é fácil ver que os abertos básicos de $\mathcal{T}_{p}$ são interseções finitas de conjuntos da forma

$$
[F, V]:=\left\{f \in Y^{X}: f[F] \subseteq V\right\}
$$


$\operatorname{com} F \in[X]^{<\omega}$ e $V \in \rho$. Denotamos por $C_{p}(X, Y)$ o conjunto $C(X, Y):=\left\{f \in Y^{X}: f\right.$ é contínua visto como subespaço de $\left(Y^{X}, \mathcal{T}_{p}\right)$ - em particular, escrevemos $C(X)$ para indicar o conjunto das funções de $X$ em $\mathbb{R}$ contínuas, e $C_{p}(X)$ indica o conjunto $C(X)$ com a topologia de subespaço oriunda de $\left(\mathbb{R}^{X}, \mathcal{T}_{p}\right)$.

Em $\mathbb{R}^{X}$, podemos considerar a topologia $\mathcal{T}_{u}$ na qual os conjuntos da forma

$$
\mathcal{U}_{n}(f):=\left\{g \in \mathbb{R}^{X}:|f(x)-g(x)|<\frac{1}{n+1}\right\}
$$

para cada $n \in \omega$ e $f \in \mathbb{R}^{X}$, constituem um sistema fundamental de vizinhanças para $f$; chamamos $\mathcal{T}_{u}$ de topologia da convergência uniforme pelo seguinte fato, cuja verificação é trivial: uma sequência $\left(f_{n}\right)_{n \in \omega}$ em $\left(\mathbb{R}^{X}, \mathcal{T}_{u}\right)$ converge para $f$ se, e somente se, $f_{n} \rightarrow f$ uniformemente (cf. Seção 1.3 .

Já podemos ilustrar certas variações no "comportamento" de $C(X)$ de acordo com a topologia adotada. Por exemplo, para uma sequência $\left(f_{n}\right)_{n \in \omega}$ em $C(X), f_{n} \rightarrow_{\mathcal{T}_{u}} f$ implica $f \in C(X)$, em outras palavras, isso significa que $C(X)$ é fechado em $\left(\mathbb{R}^{X}, \mathcal{T}_{u}\right)$. Por outro lado, o mesmo não é verdadeiro quando tomamos $C(X)$ como subespaço de $\left(\mathbb{R}^{X}, \mathcal{T}_{p}\right)$. Com efeito, para $X=\mathbb{R}$ e considerando $f_{n}: \mathbb{R} \rightarrow \mathbb{R}$ definida por $f_{n}(x)=\cos (\pi x)^{2 n}$ para quaisquer $x \in \mathbb{R}$ e $n \in \omega$, segue que $f_{n}(x) \rightarrow f(x)=\lim _{n \rightarrow \infty} \cos (\pi x)^{2 n}$ para todo $x \in \mathbb{R}$, com $f$ descontínua e $\left\{f_{n}: n \in \omega\right\} \subset C(\mathbb{R})$.

Diferentes topologias sobre $C(X)$ permitem tratar mais facilmente certas propriedades topológicas em $X$ e, muitas vezes, podemos obter traduções do tipo " $X$ tem a propriedade $\mathcal{P}$ se, e somente se, $(C(X), \mathcal{T})$ tem a propriedade $\mathcal{Q}$ ", para alguma topologia $\mathcal{T}$ sobre $C(X)$ - boa parte do Capítulo 2 trata de traduções desse tipo. Por exemplo, por meio da topologia $\mathcal{T}_{u}$ sobre $C(X)$, o Teorema de Stone-Weierstrass (enunciado logo abaixo) permite estender funções contínuas em espaços de Tychonoff] Seguindo a notação de Tkachuk, [47], diremos que $A \subseteq C(X)$ é uma álgebra se $A$ contiver todas as funções constantes de $C(X) \mathrm{e}$ $\{f+g, f \cdot g\} \subseteq A$ para quaisquer $f, g \in A$; dizemos que $A$ separa pontos em $X$ se para todo par de pontos $x, y \in X$ distintos existir $f \in A$ tal que $f(x) \neq f(y)$.

\footnotetext{
${ }^{6}$ No Capítulo 3, usaremos tais resultados juntamente com a Compactificação de Stone-Čech a fim de obter uma generalização para o Teorema de Tietze para espaços normais e, por isso, frisamos que as demonstrações do Teorema de Stone-Weierstrass e do Corolário 1.2.2 não dependem do Teorema de Tietze.
} 
Teorema 1.2.1 (Teorema de Stone-Weierstrass). Seja K um espaço compacto Hausdorff. Se $A \subseteq C(K)$ é uma álgebra que separa pontos de $K$ e $A$ é fechado em $\left(C(X), \mathcal{T}_{u}\right)$, então $A=C(K)$.

Demonstração. Uma prova completa para este teorema pode ser encontrada, por exemplo, em Tkachuk, [47], Problema 191.

Corolário 1.2.2. Sejam $X$ um espaço de Tychonoff e $K \subseteq X$ compacto. Se $f: K \rightarrow \mathbb{R} e ́$ contínua, então existe uma extensão contínua $\tilde{f}: X \rightarrow \mathbb{R}$.

Demonstração. Seja $K \subseteq X$ compacto, e chame $A=\{f \in C(K): \exists g \in C(X)(g \uparrow K=$ $f)$ \}; se mostrarmos que $A=C(K)$, então o corolário estará demonstrado. Claramente, $A$ é uma álgebra e, por $X$ ser um espaço de Tychonoff, $A$ separa pontos de $K$. Considere então $\left\{f_{n}\right\}_{n \in \omega} \subseteq A$ e $f \in C(K) \operatorname{com} f_{n} \rightarrow f$ uniformemente, provaremos que $f \in A$.

Tomando uma subsequência de $\left\{f_{n}\right\}_{n \in \omega}$ se necessário, podemos supor que $\mid f_{n+1}(x)-$ $f_{n}(x) \mid<2^{-(n+1)}$, para todo $n \in \omega$ e para todo $x \in K$. Agora, como $A$ é álgebra, para cada $n \in \omega$ temos $f_{n+1}-f_{n} \in A$ e, por conseguinte, existe $h_{n} \in C(X)$ que estende $f_{n+1}-f_{n}$. Defina

$$
g_{n}=\max \left\{\min \left\{h_{n}, 2^{-(n+1)}\right\},-2^{-(n+1)}\right\}
$$

e note que $\tilde{g}=\sum_{i=0}^{\infty} g_{n}$ é contínua em $X$ (pois $\tilde{g}$ é limite uniforme de funções contínuas) e, fazendo $g=\tilde{g}+\tilde{f}_{0}$, onde $\tilde{f}_{0} \in C(X)$ estende $f_{0}$, temos $\tilde{g}+\tilde{f}_{0} \uparrow K=f$, donde segue que $f \in A$. Como $K$ é compacto Hausdorff, pelo Teorema de Stone-Weierstrass concluímos que $A=C(K)$.

Em particular, do corolário acima segue que se $\left\{x_{0}, \ldots, x_{n}\right\} \subset X$ e $\left\{r_{0}, \ldots, r_{n}\right\} \subset \mathbb{R}$, então existe uma função contínua $f: X \rightarrow \mathbb{R}$ tal que $f\left(x_{i}\right)=r_{i}$ para todo $i \leq n$. Tal fato permite provar que $C_{p}(X)$ é denso em $\left(\mathbb{R}^{X}, \mathcal{T}_{p}\right)$ para todo espaço de Tychonoff $X$, o que deixamos a cargo do leitor. 


\subsection{Uniformidades e espaços uniformes}

O material apresentado nesta seção visa fixar algumas notações, bem como dar as definições e resultados básicos sobre uniformidades. Usaremos isso no capítulo seguinte somente para simplificar algumas verificações sobre propriedades de metrizabilidade e axiomas de separação.

Intuitivamente, o conceito de uniformidade se encontra entre as noções de métrica e topologia, o que permite a análise de diversos tópicos tipicamente "métricos" sob uma perspectiva mais geral. Em particular, espaços uniformes possuem informação suficiente para generalizar a ideia de convergência uniforme.

Antes de definirmos as uniformidades propriamente, relembramos o leitor de que se $\mathcal{U}$ e $\mathcal{V}$ são subconjuntos de $X \times X$, então os conjuntos $\mathcal{U}$ e $\mathcal{V}$ são relações binárias (conjuntos de pares ordenados) e, por conseguinte, as notações $\mathcal{U}^{-1}$ e $\mathcal{V} \circ \mathcal{U}$ têm o sentido usual, e indicam respectivamente a relação inversa de $\mathcal{U}$ e a composição entre $\mathcal{V}$ e $\mathcal{U}$ (como frisamos na Seção 1.1). Uma estrutura uniforme (ou uniformidade) sobre um conjunto $X$ é uma família $\mathfrak{U}$ de subconjuntos de $X \times X$ satisfazendo:

(U1) se $\mathcal{A} \in \mathfrak{U}$ e $\mathcal{A} \subseteq \mathcal{B} \subseteq X \times X$, então $\mathcal{B} \in \mathfrak{U}$;

(U2) a família $\mathfrak{U}$ é fechada por interseções finitas;

(U3) se $\mathcal{A} \in \mathfrak{U}$, então $\Delta \subseteq \mathcal{A}$;

(U4) se $\mathcal{V} \in \mathfrak{U}$, então existe $\mathcal{U} \in \mathfrak{U}$ tal que $\mathcal{U}^{-1} \subseteq \mathcal{V}$;

(U5) se $\mathcal{V} \in \mathfrak{U}$, então existe $\mathcal{W} \in \mathfrak{U}$ tal que $\mathcal{W} \circ \mathcal{W} \subseteq \mathcal{V}$

Em tal caso, dizemos que $(X, \mathfrak{U})$ é um espaço uniforme, e os elementos de $\mathfrak{U}$ são chamados de entourages. Se $\mathfrak{U}$ e $\mathfrak{V}$ são uniformidades sobre $X, \mathfrak{U}$ é mais fina do que $\mathfrak{V}$ se $\mathfrak{V} \subseteq \mathfrak{U}$. Uma uniformidade sobre um conjunto $X$ pode também ser definida por meio de coberturas de subconjuntos de $X$ que satisfazem certas condições 7 Se $(X, \mathfrak{U})$ é um espaço uniforme, uma base para a uniformidade $\mathfrak{U}$ é uma família $\mathfrak{B} \subseteq \mathfrak{U}$ satisfazendo:

(BU1) se $\mathcal{A} \in \mathfrak{B}$, então $\Delta \subseteq \mathcal{A}$;

\footnotetext{
${ }^{7}$ Ao leitor interessado em tal abordagem, sugerimos os textos de Engelking (Capítulo 8, [15]) e Willard (Capítulo $9,[50])$.
} 
(BU2) se $\mathcal{V}_{1}, \mathcal{V}_{2} \in \mathfrak{B}$, então existe $\mathcal{V}_{3} \in \mathfrak{B}$ tal que $\mathcal{V}_{3} \subseteq \mathcal{V}_{1} \cap \mathcal{V}_{2}$;

(BU3) se $\mathcal{V} \in \mathfrak{B}$, então existe $\mathcal{V}^{\prime} \in \mathfrak{B}$ tal que $\mathcal{V}^{\prime} \subseteq \mathcal{V}^{-1}$

(BU4) se $\mathcal{V} \in \mathfrak{B}$, então existe $\mathcal{W} \in \mathfrak{B}$ tal que $\mathcal{W} \circ \mathcal{W} \subseteq V$.

Reciprocamente, note que se $\mathfrak{B}$ é uma família satisfazendo as condições acima, então a família $\mathfrak{U}(\mathfrak{B})$ definida por $\mathfrak{U}(\mathfrak{B})=\{\mathcal{A} \subseteq X \times X: \exists \mathcal{V} \in \mathfrak{B}(\mathcal{V} \subseteq \mathcal{A})\}$ é uma uniformidade sobre $X$. Além disso, $\mathfrak{B}$ é uma base de $\mathfrak{U}(\mathfrak{B})$.

Dado um espaço uniforme $(X, \mathfrak{U})$, podemos induzir uma topologia $\tau(X, \mathfrak{U})$ sobre $X$ declarando um subconjunto qualquer $A$ de $X$ como aberto se para todo $x \in A$ existir $\mathcal{U} \in \mathfrak{U}$ tal que $\mathcal{U}[x] \subseteq A$, onde $\mathcal{U}[x]:=\{y \in X:(x, y) \in \mathcal{U}\}$; como $\mathfrak{U}$ é fechada por interseções finitas, segue que $\tau(X, \mathfrak{U})$ é de fato uma topologia sobre $X$. Evidentemente, se $\mathfrak{B}$ for uma base para $\mathfrak{U}$, então basta utilizarmos os elementos de $\mathfrak{B}$ na definição acima. Mais geralmente, vale a seguinte proposição:

Proposição 1.3.1. Sejam $\mathfrak{U}, \mathfrak{V}$ uniformidades sobre $X$ e $\mathfrak{B}_{0}, \mathfrak{B}_{\perp}$ bases fixadas para $\mathfrak{U}$ e $\mathfrak{V}$, respectivamente. A uniformidade $\mathfrak{U}$ é mais fina do que $\mathfrak{V} s e$, e somente se, para todo $\mathcal{U} \in \mathfrak{B}_{0}$ existe $\mathcal{V} \in \mathfrak{B}_{1}$ tal que $\mathcal{V} \subseteq \mathcal{U}$. Além disso, se $\mathfrak{U} \subseteq \mathfrak{V}$, então $\tau_{0}=\tau(X, \mathfrak{U}) \subseteq \tau(X, \mathfrak{V})=\tau_{1}$

Demonstração. A primeira parte decorre diretamente da condição (U1) e da definição de base para uniformidade. Enfim, supondo $\mathfrak{U} \subseteq \mathfrak{V}$, note que se $A \subseteq X$ é um $\tau_{0}$-aberto, então para todo $x \in A$ existe $\mathcal{U} \in \mathfrak{U} \subseteq \mathfrak{V}$ tal que $\mathcal{U}[x] \subseteq A$, e assim $A$ é um $\tau_{1}$-aberto.

Em particular, se $Y \subseteq X$, então $\mathfrak{U}_{Y}=\{U \cap(Y \times Y): U \in \mathfrak{U}\}$ é uma uniformidade sobre $Y$, cuja topologia induzida coincide com a topologia de $Y$ como subespaço de $(X, \tau(X, \mathfrak{U}))$.

Pelo restante desta seção, $(X, \mathfrak{U})$ é um espaço uniforme fixado e $\tau=\tau(X, \mathfrak{U})$ é a topologia sobre $X$ induzida por $\mathfrak{U}$. Em [15], o autor utiliza a notação $d(x, y)<\mathcal{U}$ a fim de indicar que $(x, y) \in \mathcal{U}$, onde $\mathcal{U}$ é uma entourage de $X$. Isso sugere que uma uniformidade se comporta de forma semelhante a uma métrica em $X$, o que de fato ocorre. Com efeito, dado um espaço métrico $(M, \delta)$, se denotarmos por

$$
\mathcal{V}_{\varepsilon}=\{(x, y) \in M \times M: \delta(x, y)<\varepsilon\}
$$


então $\mathfrak{B}_{\mathcal{V}}=\left\{\mathcal{V}_{\varepsilon}: \varepsilon>0\right\}$ é base para uma uniformidade $\mathfrak{U}(\delta)$ sobre $M$ - em particular, as condições $\left(\mathrm{BU} 3 \mathrm{3}\right.$ e $\left(\mathrm{BU} 4 \mathrm{4}\right.$ se verificam pois $\mathcal{V}_{\varepsilon}=\mathcal{V}_{\varepsilon}^{-1}$ e $\mathcal{V}_{\frac{\varepsilon}{2}} \circ \mathcal{V}_{\frac{\varepsilon}{2}} \subseteq \mathcal{V}_{\varepsilon}$ para qualquer $\varepsilon>0$ Como temos $\mathcal{V}_{\varepsilon}[x]=B_{\delta}(x, \varepsilon)$ - a bola aberta de centro $x$ e raio $\varepsilon-$, segue que a topologia em $M$ induzida pela métrica $\delta$ coincide com a topologia em $M$ induzida por $\mathfrak{U}(\delta)$.

Contudo, vale frisar que dada uma entourage $\mathcal{U}$ de $X$, não é necessariamente verdadeiro que $\mathcal{U}[x]$ seja aberto em $X$ na topologia induzida por $\mathfrak{U}$. Como exemplo, basta considerarmos o mesmo espaço métrico $(M, \delta)$ e definirmos os conjuntos $\mathcal{U}_{\varepsilon}=\{(x, y) \in M \times M: \delta(x, y) \leq$ $\varepsilon\}$, os quais induzem uma base para uma uniformidade $\mathfrak{U}^{\prime}(\delta)$ sobre $X$ que coincide com a uniformidade $\mathfrak{U}(\delta)$ definida no parágrafo anterior, porém, neste caso temos $\mathcal{U}_{\varepsilon}[x]=B_{\delta}[x, \varepsilon]-$ a bola fechada de centro $x$ e raio $\varepsilon$-, que não é necessariamente um aberto de $M$. Ainda assim, é sempre verdade que $\mathcal{U}[x]$ é uma vizinhança de $x$, como formalizamos a seguir.

Proposição 1.3.2. Para $A \subseteq X$ qualquer, vale a identidade int $(A)=\{x \in X: \exists \mathcal{U} \in$ $\mathfrak{U}(\mathcal{U}[x] \subseteq A)\}$. Em particular, $\mathcal{U}[x]$ é uma vizinhança de $x$, para quaisquer $x \in X$ e $\mathcal{U} \in \mathfrak{U}$.

Demonstração. Por simplicidade, chamemos $\{x \in X: \exists \mathcal{U} \in \mathfrak{U}(\mathcal{U}[x] \subseteq A)\}=W$. Pela definição de $\tau$, se $G \subseteq A$ é aberto, então $G \subseteq W$. Assim, para provar a igualdade desejada, basta mostrarmos que $W$ é aberto. Para tanto, sejam $w \in W$ e $\mathcal{U} \in \mathfrak{U}$ tais que $\mathcal{U}[w] \subseteq A$. Como $\mathfrak{U}$ é uniformidade, segue que existe $\mathcal{V} \in \mathfrak{U}$ satisfazendo $\mathcal{V} \circ \mathcal{V} \subseteq \mathcal{U}$. Se mostrarmos que para qualquer $u \in \mathcal{V}[w]$ vale $\mathcal{V}[u] \subseteq A$ (isto é, $\mathcal{V}[w] \subseteq W$ ), então a proposição estará demonstrada. Agora, se $x \in \mathcal{V}[u]$ e $u \in \mathcal{V}[w]$, então $(w, u),(u, x) \in \mathcal{V}$, o que acarreta $(w, x) \in \mathcal{V} \circ \mathcal{V} \subseteq \mathcal{U}$ e, consequentemente, $x \in \mathcal{U}[w] \subseteq A$. Logo, $\mathcal{V}[u] \subseteq A$. Em particular, como $x \in \mathcal{U}[x] \subseteq \mathcal{U}[x]$, concluímos que $x \in \operatorname{int}(\mathcal{U}[x]) \subseteq \mathcal{U}[x]$.

Para uma net $\left(x_{l}\right)_{l \in L}$ em $X$ e $x \in X$, a condição de convergência em $\tau$ se traduz da seguinte maneira: $x_{l} \rightarrow_{\tau} x$ se, e somente se, para toda entourage $\mathcal{U} \in \mathfrak{U}$ existe $l_{\mathcal{U}} \in L$ tal que $x_{l} \in \mathcal{U}[x]$ para todo $l \geq l_{\mathcal{U}}$. Mais geralmente, dado um conjunto $W$, uma net de funções $\left(f_{l}\right)_{l \in L}$ em $X^{W}$ e $f \in X^{W}$, dizemos que $f_{l} \rightarrow f$ uniformemente se para quaisquer $w \in W$ e $\mathcal{U} \in \mathfrak{U}$ existe $l_{0} \in L$ tal que $f_{l}(w) \in \mathcal{U}[f(w)]$ para todo $l \geq l_{0}$. Assim, espaços uniformes possuem estrutura suficiente para generalizarmos a noção de convergência uniforme - de modo semelhante, é possível adaptar a definição de sequência de Cauchy do contexto métrico para uniformidades. 
Além disso, a classe dos espaços uniformes coincide com a classe dos espaços de Tychonoff, no seguinte sentido:

Proposição 1.3.3. (a) A topologia $\tau(X, \mathfrak{U})$ induzida por $\mathfrak{U}$ é de Hausdorff se, e somente se, $\bigcap \mathfrak{U}=\Delta$.

(b) Um espaço topológico $(X, \tau)$ é $T_{3 \frac{1}{2}}$ se, e somente se, $\tau=\tau(X, \mathfrak{U})$ para alguma uniformidade $\mathfrak{U}$. Em particular, $\bigcap \mathfrak{U}=\Delta$ se, e somente se, $(X, \tau(X, \mathfrak{U}))$ é um espaço de Tychonoff.

Demonstração. A verificação do item (a) segue diretamente da definição, aliado ao fato de que as entourages simétricas constituem uma base para $\mathfrak{U}$ ( $c f$. item $(b)$ do Lema 1.3.7). Uma demonstração completa para o item $(b)$ pode ser encontrada, por exemplo, em [50], Teorema 38.2 .

Dizemos que $\mathfrak{U}$ é uma uniformidade metrizável caso exista uma métrica $\delta$ sobre $X$ satisfazendo $\mathfrak{U}(\delta)=\mathfrak{U}$, onde $\mathfrak{U}(\delta)$ é gerada pelos conjuntos da forma $\mathcal{V}_{\varepsilon}$ definidos em 1.3.1. Analogamente, se $\mathfrak{U}(\delta)=\mathfrak{U}$ para alguma pseudo-métrica $\delta$ sobre $X$, diremos que $\mathfrak{U}$ é uma uniformidade pseudo-metrizável. Vale então o seguinte critério:

Proposição 1.3.4. Uma uniformidade $\mathfrak{U}$ sobre $X$ é pseudo-metrizável se, e somente se, $\mathfrak{U}$ tem uma base enumerável.

Demonstração. Willard apresenta uma prova completa em [50], Teorema 38.3.

Em particular, como a topologia induzida por uma pseudo-métrica $\delta$ é $T_{1}$ se, e somente se, $\delta$ é uma métrica, o resultado anterior aliado ao item (a) da Proposição 1.3 .3 nos dá o corolário abaixo.

Corolário 1.3.5. Uma uniformidade $\mathfrak{U}$ sobre $X$ é metrizável se, e somente se, $\bigcap \mathfrak{U}=\Delta$ e $\mathfrak{U}$ tem base enumerável.

Os dois próximos lemas nos apresentam resultados que serão úteis adiante. O primeiro deles, que também ilustra as semelhanças entre espaços métricos e espaços uniformes, é uma generalização do seguinte fato para espaços métricos? se um compacto $K$ está contido num

\footnotetext{
${ }^{8}$ Teorema da Cobertura de Lebesgue [15], em outras referências também chamado de Teorema do Número de Lebesgue.
} 
aberto $V$, então existe $r>0$ tal que $K^{r}:=\bigcup_{x \in K} B(x, r) \subseteq V$. O segundo Lema é meramente um resultado técnico. Para um subconjunto $A$ de $X$ e uma entourage $\mathcal{U}$ de $X$, definimos $\mathcal{U}[A]:=\bigcup_{a \in A} \mathcal{U}[a]$.

Lema 1.3.6. Seja $K \subseteq X$ um compacto na topologia $\tau=\tau(X, \mathfrak{U})$. Se $V$ é um aberto tal que $K \subseteq V$, então existe $\mathcal{U} \in \mathfrak{U}$ satisfazendo $\mathcal{U}[K] \subseteq V$

Demonstração. Por $V$ ser aberto, para cada $x \in K \subseteq V$ existe $\mathcal{V}_{x} \in \mathfrak{U}$ tal que $\mathcal{V}_{x}[x] \subseteq V$, donde tomamos $\mathcal{W}_{x} \in \mathfrak{U} \operatorname{com} \mathcal{W}_{x} \circ \mathcal{W}_{x} \subseteq \mathcal{V}_{x}$. Como $\left\{\mathcal{W}_{x}[x]: x \in K\right\}$ é uma cobertura de vizinhanças para $K$, existem $x_{0}, \ldots, x_{n} \in K$ tais que $K \subseteq \bigcup_{i \leq n} \mathcal{W}_{x_{i}}\left[x_{i}\right]$.

Afirmamos que $\mathcal{U}=\bigcap_{i \leq n} \mathcal{W}_{x_{i}}$ satisfaz o enunciado proposto. De fato, se $x \in \mathcal{U}[K]$, então $x \in \mathcal{U}[y]$ para algum $y \in K$, e por sua vez $y \in \mathcal{W}_{x_{i}}\left[x_{i}\right]$ para algum $i \leq n$. Assim, temos $\left(x_{i}, y\right) \in \mathcal{W}_{x_{i}}$ e $(y, x) \in \mathcal{U} \subseteq \mathcal{W}_{x_{i}}$, i.e., $\left(x_{i}, x\right) \in \mathcal{W}_{x_{i}} \circ \mathcal{W}_{x_{i}} \subseteq \mathcal{V}_{x_{i}}$ e, finalmente, $x \in \mathcal{V}_{x_{i}}\left[x_{i}\right] \subseteq V$

Lema 1.3.7. Considere $X$ munido da topologia $\tau=\tau(X, \mathfrak{U})$ e $X \times X$ com a topologia produto.

(a) Para todo $\mathcal{U} \in \mathfrak{U}$, existe $\mathcal{V} \in \mathfrak{U}$ tal que $\mathcal{V} \circ \mathcal{V} \circ \mathcal{V} \subseteq \mathcal{U}$;

(b) O conjunto $\mathcal{S}(\mathfrak{U})=\left\{\mathcal{V} \in \mathfrak{U}: \mathcal{V}=\mathcal{V}^{-1}\right\}$ é uma base para $\mathcal{U}$ (os elementos $\mathcal{S}(\mathfrak{U})$ são chamados de entourages simétricas).

(c) $\mathfrak{O}_{s}(\mathfrak{U})=\{\mathcal{V} \in \mathcal{S}(\mathfrak{U}): \mathcal{V}$ é aberto em $X \times X\}$ é uma base para $\mathfrak{U}$.

Demonstração. Para o item (a) basta aplicar duas vezes a condição (U5) de uniformidade e uma vez a condição (U2). Para (b), as condições (U1) e (U4) garantem que se $\mathcal{U} \in \mathfrak{U}$, então $\mathcal{U}^{-1} \in \mathfrak{U}$ e, assim, por (U2) temos $\mathcal{U} \cap \mathcal{U}^{-1} \in \mathfrak{U}$, que é um membro de $\mathcal{S}(\mathfrak{U})$ contido em $\mathcal{U}$. Para o item $(c)$, note primeiramente que se $\mathcal{V} \in \mathfrak{U}$ é aberto em $X \times X$, então $\mathcal{V}^{-1}$ também é aberto, e daí $\mathcal{V} \cap \mathcal{V}^{-1}$ é um aberto simétrico; desse modo, é suficiente mostrar que se $\mathcal{V} \in \mathfrak{U}$, então $\operatorname{int}(\mathcal{V}) \in \mathfrak{U}$, isto é, as entourages abertas de $X$ constituem uma base para $\mathfrak{U}$. Para provar isto, tome $\mathcal{U} \in \mathcal{S}(\mathfrak{U})$ satisfazendo $\mathcal{U} \circ \mathcal{U} \circ \mathcal{U} \subseteq \mathcal{V}$ e observe que $\mathcal{U} \subseteq \operatorname{int}(\mathcal{V})$.

Vamos apresentar mais duas topologias para espaços de funções. Considere fixados espaços topológicos $(X, \tau)$ e $(Y, \rho)$. A topologia compacto-aberta é a topologia $\tau_{k}$ sobre $C(X, Y)$ 
cujos abertos básicos são interseções finitas de conjuntos da forma

$$
[K, V]:=\{f \in C(X, Y): f[K] \subseteq V\}
$$

para quaisquer $K \subseteq X$ compacto e $V \in \sigma$ (compare com 1.2.2)). Indicamos por $C_{k}(X, Y)$ o conjunto $C(X, Y)$ munido da topologia compacto-aberta, ou simplesmente por $C_{k}(X)$ quando $Y=\mathbb{R}$. Adiamos a definição dessa topologia para a atual seção pois, quando $Y$ é um espaço uniforme, digamos que com uma uniformidade $\mathfrak{U}$, e $X$ é de Hausdorff, $C_{k}(X, Y)$ é subespaço de $\left(Y^{X}, \mathcal{T}_{k}\right)$, onde $\mathcal{T}_{k}$ é uma topologia sobre $Y^{X}$ gerada por uma uniformidade cujas entourages básicas são da forma

$$
\langle K, \mathcal{U}\rangle:=\left\{(f, g) \in Y^{X} \times Y^{X}:(f(x), g(x)) \in \mathcal{U}, \forall x \in K\right\}
$$

para cada $K \subseteq X$ compato e $\mathcal{U} \in \mathfrak{U}$. Chamamos a topologia $\mathcal{T}_{k}$ de topologia da convergência uniforme em compactos por um motivo análogo ao da topologia da convergência pontual: uma net $\left(f_{l}\right)_{l \in L}$ em $Y^{X}$ converge para $f$ na topologia $\mathcal{T}_{k}$ se, e somente se, $f_{l} \uparrow K \rightarrow f \uparrow K$ uniformemente para todo $K \subseteq X$ compacto.

Provaremos tais afirmações num contexto mais geral no Capítulo 2. Em particular, analisaremos de forma simultânea as topologias $\mathcal{T}_{p}$ e $\mathcal{T}_{k}$ sobre $C(X)$, enxergando ambas as topologias como casos particulares de uma classe de topologias geradas por meio de certas uniformidades em $C(X)$. 


\subsection{Jogos topológicos seletivos}

O termo jogo topológico foi inicialmente introduzido por Berge em 1957, embora o conceito de jogo num contexto matemático geral já seja usado desde o início do século XVII. Ao leitor interessado nos aspectos históricos do desenvolvimento dos jogos topológicos, bem como em outros tipos de jogos topológicos diferentes daqueles que mostraremos, sugerimos o texto de Telgársky [46]. Nesta seção, faremos uma breve apresentação sobre jogos seletivos e princípios de seleção; baseamo-nos fortemente nos artigos de Scheepers [39, 40, 41] e num minicurso sobre jogos topológicos [11], ministrado por Dias em janeiro de 2013 no ICMC.

A importância de tal abordagem para a topologia não está no jogo em si, mas sim na existência (ou não existência) de uma estratégia vencedora para algum dos jogadores, o que varia de acordo com as propriedades topológicas do espaço no qual o jogo é realizado. Neste trabalho, um jogo topológico $G$ num espaço topológico $X$ consiste, grosso modo, numa partida entre dois jogadores - os jogadores I e II -, composta por infinitas rodadas, nas quais o jogador I escolhe algum objeto $P$ de uma classe $\mathcal{P}$ de objetos relacionados a um espaço topológico, e o jogador II responde escolhendo um objeto $Q$ relacionado a $P$; o critério de vitória consiste então de alguma condição exigida sobre os objetos $P$ e $Q$ escolhidos pelos jogadores I e II.

Mais precisamente, fixados um ordinal $\alpha \geq \omega$ e famílias $\mathfrak{U}, \mathfrak{V}$ de conjuntos não vazios, denotaremos por $\mathrm{G}_{1}^{\alpha}(\mathfrak{U}, \mathfrak{V})$ o jogo de comprimento $\alpha$ entre os jogadores I e II, no qual uma partida é composta por $\alpha$ rodadas (ou turnos); em cada rodada $\beta<\alpha$, o jogador I escolhe um elemento $U_{\beta} \in \mathfrak{U}$, em seguida o jogador II responde escolhendo um elemento $u_{\beta} \in U_{\beta}$, o que encerra o turno $\beta$; a partida é vencida pelo jogador II se, e somente se, $\left\{u_{\beta}: \beta<\alpha\right\} \in \mathfrak{V}$. Quando $\alpha=\omega$, denotamos simplesmente por $\mathrm{G}_{1}(\mathfrak{U}, \mathfrak{V})$.

Intuitivamente, chamamos de estratégia a maneira com que cada jogador faz suas escolhas. Ou seja, uma estratégia para o jogador I (ou para o jogador II) é uma regra que diz a ele como fazer suas jogadas levando em consideração os movimentos já realizados pelos dois jogadores. Assumiremos que ambos os jogadores têm memória infinita (ou ilimitada), no sentido de que eles se "lembram" de todos os movimentos anteriores no momento de realizarem suas jogadas. Além disso, é importante frisar que a estratégia de um jogador independe da estratégia de seu adversário, em outras palavras, como o jogador I não sabe qual será a resposta do jogador II, 
a estratégia do jogador I deve estar "preparada" para qualquer resposta legal do jogador II, e vice-versa.

"Simularemos" uma partida entre os jogadores I e II no jogo $\mathrm{G}_{1}^{\alpha}(\mathfrak{U}, \mathfrak{V})$ para tornarmos tais conceitos mais claros e assim entendermos a eurística por trás da definição formal de estratégia que adotaremos. Denotaremos por $\sigma$ a estratégia do jogador I, e por $\mu$ a estratégia do jogador II.

- Turno 0: o jogador I escolhe algum $U_{0} \in \mathfrak{U}$ e o jogador II responde com algum $u_{0} \in U_{0}$. Neste turno, não há jogadas realizadas anteriomente e o jogador I precisa fazer seu 0ésimo lance. Logo, podemos considerar sua escolha $U_{0}$ como sendo uma "resposta" à sequência vazia, isto é, $\sigma(\emptyset)=U_{0}$ é o 0-ésimo lance do jogador I pela estratégia $\sigma$. Por sua vez, o jogador II já conhece o lance $U_{0}$ do jogador I, de modo que sua resposta deve ser um elemento de $U_{0}$ (do contrário, a escolha do jogador II não seria um movimento válido). Assim, $\mu\left(\left(U_{0}\right)\right)=u_{0} \in U_{0}$ é o 0-ésimo lance do jogador II de acordo com a estratégia $\mu$.

- Turno 1: o jogador I escolhe algum $U_{1} \in \mathfrak{U}$ e o jogador II responde com algum $u_{1} \in U_{1}$. Neste momento, o jogador I sabe que na rodada anterior ele jogou $U_{0} \in \mathfrak{U}$ e o jogador II respondeu com $u_{0} \in U_{0}$. Num primeiro momento, isso nos faz considerar o lance $U_{1}$ como uma resposta à sequência $\left(U_{0}, u_{0}\right)$, i.e., $\sigma\left(\left(U_{0}, u_{0}\right)\right)=U_{1}$. Contudo, é suficiente que o jogador I se lembre apenas da resposta do jogador II, pois $\sigma$ já "sabe" que sua resposta ao conjunto $\emptyset$ é $U_{0}$ e, por definição, o lance $u_{0}$ do jogador II é uma resposta a $U_{0}$. Dessa forma, se no momento de decidir seu 1-ésimo lance o jogador I recebe apenas a sequência $\left(u_{0}\right)$, é possível obter a sequência $\left(U_{0}, u_{0}\right)$ por meio de $\sigma$, de modo que não há "perda de informação". Por tal motivo, diremos que $\sigma\left(\left(u_{0}\right)\right)=U_{1}$ é o 1-ésimo lance do jogador I. Analogamente, o 1-ésimo movimento do jogador II é uma resposta a $\left(U_{0}, U_{1}\right)$, pois com tal informação recupera-se por meio de $\mu$ a sequência $\left(U_{0}, u_{0}, U_{1}\right)$; $\operatorname{assim} \mu\left(\left(U_{0}, U_{1}\right)\right)=u_{1}$.

- Turno $\beta<\alpha$ : o jogador I escolhe algum $U_{\beta} \in \mathfrak{U}$ e o jogador II responde com algum $u_{\beta} \in U_{\beta}$. O jogador I inicia o turno $\beta$, e para escolher $U_{\beta}$ ele tem acesso a todos os lances anteriores, tanto os dele quanto os do adversário. Novamente, como no caso anterior, 
basta que o jogador I "avalie" a sequência $\left(u_{\gamma}: \gamma<\beta\right)$ das respostas de seu adversário, onde $u_{\gamma}$ foi o lance do jogador II no turno $\gamma<\beta$; desse modo, recursivamente o jogador I sabe que até agora os lances foram $U_{0}, u_{0}, U_{1}, u_{1}, \ldots, U_{\gamma}, u_{\gamma}, \ldots$, e pode enfim escolher $U_{\beta} \in \mathfrak{U}$. Dizemos então que $\sigma\left(\left(u_{\gamma}: \gamma<\beta\right)\right)=U_{\beta}$ é o $\beta$-ésimo movimento do jogador I. De maneira inteiramente análoga, o $\beta$-ésimo movimento do jogador II é da forma $\mu\left(\left(U_{\gamma}\right.\right.$ : $\gamma \leq \beta))=u_{\beta}$ - note que, neste caso, fazemos $\gamma \leq \beta$ pois o jogador II já conhece o lance $U_{\beta}$ realizado neste turno.

A descrição acima deixa claro que tanto $\sigma$ quanto $\mu$ são funções: $\sigma$ recebe como entrada certas sequências da forma $\left(u_{\gamma}: \gamma<\beta\right) \in(\bigcup \mathfrak{U})^{<\alpha}$ e retorna elementos da forma $U_{\beta} \in \mathfrak{U}$; por sua vez, para uma sequência $\left(U_{\gamma}: \gamma \leq \beta\right) \in \mathfrak{U}^{<\alpha} \backslash\{\emptyset\}, \mu$ associa um elemento $u_{\beta} \in U_{\beta} \subseteq \bigcup \mathfrak{U}$. Note que do "ponto de vista" do jogador II, a cada turno $\beta<\alpha$ ele precisa saber como responder a qualquer $U \in \mathfrak{U}$ escolhido pelo jogador I como sendo $U_{\beta}$, e disso segue que o domínio de $\mu$ é $\mathfrak{U}^{<\alpha} \backslash\{\emptyset\}$. Porém, $\sigma$ reconhece somente as sequências $\left(u_{\gamma}: \gamma<\beta\right) \in(\bigcup \mathfrak{U})^{<\alpha}$ com $u_{\gamma} \in \sigma\left(\left(u_{\xi}: \xi<\gamma\right)\right)$ para quaisquer $\gamma<\beta<\alpha$, pois sequências que não satisfazem tal condição não representam uma sequência de movimentos válidos para o jogador II. Isso torna a definição do domínio de $\sigma$ (da forma como a obtivemos) relativamente complexa se quisermos expressá-la completamente como uma função.

Uma das alternativas seria considerar $\sigma$ como sendo uma árvore de raiz $\sigma(\emptyset)$, cujas ramificações são determinadas pelas possíveis respostas do jogador II, coisa que não faremos. Optamos por fixar um elemento $\tilde{U} \in \mathfrak{U}$ qualquer e definir $\sigma\left(\left(u_{\gamma}: \gamma<\beta\right)\right)=\tilde{U}$ para toda sequência $\left(u_{\gamma}: \gamma<\beta\right)$ que for inválida no sentido expresso acima, o que torna $\sigma$ uma função de domínio $(\bigcup \mathfrak{U})^{<\alpha}$ e não altera sua utilidade como estratégia para o jogador I, pois as novas entradas adicionadas ao domínio de $\sigma$ não serão utilizadas?

Assim, dizemos que uma estratégia para o jogador I em $G_{1}^{\alpha}(\mathfrak{U}, \mathfrak{V})$ é uma função

$$
\sigma:(\bigcup \mathfrak{U})^{<\alpha} \rightarrow \mathfrak{U}
$$

\footnotetext{
${ }^{9}$ Assumimos implicitamente que ambos os jogadores jogam estritamente de acordo com as regras.
} 
e uma estratégia para o jogador II em $\mathrm{G}_{1}^{\alpha}(\mathfrak{U}, \mathfrak{V})$ é uma função

$$
\mu:\left(\mathfrak{U}^{<\alpha} \backslash\{\emptyset\}\right) \rightarrow \bigcup \mathfrak{U}
$$

tal que $\mu\left(\left(U_{\gamma}: \gamma \leq \beta\right)\right) \in U_{\beta}$ para toda sequência $\left(U_{\gamma}\right)_{\gamma \leq \beta} \in \mathfrak{U}^{<\alpha} \backslash\{\emptyset\}$.

Agora que definimos formalmente a noção de estratégia, podemos discutir o conceito de estratégia vencedora. Por simplicidade chamaremos $G=\mathrm{G}_{1}^{\alpha}(\mathfrak{U}, \mathfrak{V})$. Fixemos estratégias $\sigma$ e $\mu$ no jogo $G$ para os jogadores I e II, respectivamente. Primeiramente, notemos que pela forma como definimos $G$, não ocorrem empates: o jogador I ganha se, e somente se, o jogador II perde. Uma sequência $P=\left(\left(U_{\beta}, u_{\beta}\right): \beta<\alpha\right)$ com $u_{\beta} \in U_{\beta} \in \mathfrak{U}$ para todo $\beta<\alpha$ será chamada de partida em $G$, onde entendemos que na rodada $\beta, U_{\beta}$ é a escolha do jogador I e $u_{\beta}$ é a resposta do jogador II; diremos que $P$ é uma partida jogada em $\sigma$ se $U_{\beta}=\sigma\left(\left(u_{\gamma}: \gamma<\beta\right)\right)$ para todo $\beta<\alpha$; $P$ é uma partida jogada em $\mu$ se $u_{\beta}=\mu\left(\left(U_{\gamma}: \gamma \leq \beta\right)\right)$ para todo $\beta<\alpha$.

Dizemos que $\sigma$ é uma estratégia vencedora para o jogador I se toda partida $P$ jogada em $\sigma$ for vencida pelo jogador I; analogamente, $\mu$ é uma estratégia vencedora para o jogador II se toda partida $P$ jogada em $\mu$ for vencida pelo jogador II. A notação $\mathrm{I} \uparrow \mathrm{G}_{1}^{\alpha}(\mathfrak{U}, \mathfrak{V})$ indica que “existe uma estratégia vencedora para o jogador I", enquanto I $\ngtr G_{1}^{\alpha}(\mathfrak{U}, \mathfrak{V})$ abrevia sua negação - adotamos notações semelhantes para o jogador II.

Notemos que I $\ngtr \mathrm{G}_{1}^{\alpha}(\mathfrak{U}, \mathfrak{V})$ é equivalente à afirmação: para qualquer estratégia $\sigma$ para o jogador I, existe uma partida $P$ jogada em $\sigma$ tal que o jogador II vence a partida $P^{10}$. Da mesma maneira, II $\gamma \mathrm{G}_{1}^{\alpha}(\mathfrak{U}, \mathfrak{V})$ é equivalente a existir uma partida $P$ jogada em $\mu$ tal que o jogador I vence a partida $P$, para qualquer estratégia $\mu$ para o jogador II. Chamamos a atenção do leitor para o seguinte fato: I $\not \mathrm{G}_{1}^{\alpha}(\mathfrak{U}, \mathfrak{V})$ e II $\not \mathrm{G}_{1}^{\alpha}(\mathfrak{U}, \mathfrak{V})$ podem ocorrer simultaneamente; em tal situação, dizemos que o jogo $G_{1}^{\alpha}(\mathfrak{U}, \mathfrak{V})$ é indeterminado .

Suponha agora II $\uparrow \mathrm{G}_{1}^{\alpha}(\mathfrak{U}, \mathfrak{V})$ e fixe uma sequência $\left(U_{\beta}\right)_{\beta<\alpha} \in \mathfrak{U}^{\alpha}$. Para cada $\beta<\alpha$ podemos obter $u_{\beta} \in U_{\beta}$ tais que $\left\{u_{\beta}: \beta<\alpha\right\} \in \mathfrak{V}$, basta tomar uma estratégia $\mu$ vencedora para o jogador II e fazer $u_{\beta}=\mu\left(\left(U_{\gamma}: \gamma \leq \beta\right)\right)$, donde segue que $P=\left(\left(U_{\beta}, u_{\beta}\right): \beta<\alpha\right)$ é uma partida em $\mu$ vencida pelo jogador II. O ponto importante a se destacar é que a recíproca não é necessariamente verdadeira, o que explicamos adiante.

\footnotetext{
${ }^{10}$ Ao leitor familiarizado com a noção de árvores: quando consideramos a estratégia do jogador I como uma árvore, intuitivamente isso significa que existe um ramo na árvore que pertence à família $\mathfrak{V}$.
} 
Indicaremos por $\mathrm{S}_{1}^{\alpha}(\mathfrak{U}, \mathfrak{V})$ a seguinte asserção: para toda sequência $\left(U_{\beta}\right)_{\beta<\alpha} \in \mathfrak{U}^{\alpha}$ existe uma sequência $\left(u_{\beta}\right)_{\beta<\alpha}$, com $u_{\beta} \in U_{\beta}$ para todo $\beta<\alpha$, tal que $\left\{u_{\beta}: \beta<\alpha\right\} \in \mathfrak{V}$. Em síntese, no parágrafo anterior afirmamos que II $\uparrow \mathrm{G}_{1}^{\alpha}(\mathfrak{U}, \mathfrak{V}) \Rightarrow \mathrm{S}_{1}^{\alpha}(\mathfrak{U}, \mathfrak{V})$. Porém, tomar como hipótese $\mathrm{S}_{1}^{\alpha}(\mathfrak{U}, \mathfrak{V})$ não garante necessariamente que o jogador II tenha uma estratégia vencedora: grosso modo, o jogador II precisaria saber de antemão qual seria a sequência de todos os movimentos do jogador I na partida, contudo, os movimentos do jogador I dependem das respostas que ele receberá do jogador II.

Já a negação de $S_{1}^{\alpha}(\mathfrak{U}, \mathfrak{V})$ implica $\mathrm{I} \uparrow \mathrm{G}_{1}^{\alpha}(\mathfrak{U}, \mathfrak{V})$ : como existe uma sequência $\left(U_{\beta}\right)_{\beta<\alpha}$ tal que para toda sequência $\left(u_{\beta}\right)_{\beta<\alpha} \operatorname{com} u_{\beta} \in U_{\beta}$ vale $\left\{u_{\beta}: \beta<\alpha\right\} \notin \mathfrak{V}$, uma estratégia vencedora para o jogador I consiste em jogar $U_{\beta}$ no turno $\beta$. Como I $\uparrow \mathrm{G}_{1}^{\alpha}(\mathfrak{U}, \mathfrak{V}) \Rightarrow$ II $\ngtr \mathrm{G}_{1}^{\alpha}(\mathfrak{U}, \mathfrak{V})$, obtemos pela contrapositiva a seguinte cadeia de implicações:

$$
\mathrm{II} \uparrow \mathrm{G}_{1}^{\alpha}(\mathfrak{U}, \mathfrak{V}) \Rightarrow \mathrm{I} \not \mathrm{G}_{1}^{\alpha}(\mathfrak{U}, \mathfrak{V}) \Rightarrow \mathrm{S}_{1}^{\alpha}(\mathfrak{U}, \mathfrak{V})
$$

Uma variação usual do jogo $G_{1}^{\alpha}(\mathfrak{U}, \mathfrak{V})$ consiste em alterar a quantidade de elementos que o jogador II pode escolher a cada turno, enquanto as regras para o jogador I continuam as mesmas. Denotamos por $\mathrm{G}_{\text {fin }}^{\alpha}(\mathfrak{U}, \mathfrak{V})$ o jogo de comprimento $\alpha$ jogado de maneira análoga ao jogo $\mathrm{G}_{1}^{\alpha}(\mathfrak{U}, \mathfrak{V})$, mas no qual a cada turno $\beta<\alpha$ o jogador II pode selecionar um subconjunto finito $V_{\beta}$ do elemento $U_{\beta}$ escolhido pelo jogador I; declaramos a partida vencida pelo jogador II se, e somente se, $\bigcup_{\beta<\alpha} V_{\beta} \in \mathfrak{V}$. Também denotamos por $\mathrm{S}_{f i n}^{\alpha}(\mathfrak{U}, \mathfrak{V})$ a variação (feita de maneira semelhante) do princípio de seleção $S_{1}^{\alpha}(\mathfrak{U}, \mathfrak{V})$. Do mesmo modo como fizemos no jogo $\mathrm{G}_{1}^{\alpha}(\mathfrak{U}, \mathfrak{V})$, definimos os conceitos de estratégia e estratégia vencedora para o jogo $\mathrm{G}_{f i n}^{\alpha}(\mathfrak{U}, \mathfrak{V})$, mutadis mutandis. Em particular, temos

$$
\mathrm{II} \uparrow \mathrm{G}_{f i n}^{\alpha}(\mathfrak{U}, \mathfrak{V}) \Rightarrow \mathrm{I} \ngtr \mathrm{G}_{f i n}^{\alpha}(\mathfrak{U}, \mathfrak{V}) \Rightarrow \mathrm{S}_{\text {fin }}^{\alpha}(\mathfrak{U}, \mathfrak{V})
$$

Pela definição dos jogos $\mathrm{G}_{1}^{\alpha}(\mathfrak{U}, \mathfrak{V})$ e $\mathrm{G}_{f i n}^{\alpha}(\mathfrak{U}, \mathfrak{V})$, segue que se $\sigma$ é uma estratégia (vencedora) para o jogador I em $\mathrm{G}_{\text {fin }}^{\alpha}(\mathfrak{U}, \mathfrak{V})$, então $\sigma$ induz uma estratégia (vencedora) para o jogador I em $G_{1}^{\alpha}(\mathfrak{U}, \mathfrak{V})$, pois o domínio de uma estratégia para o jogador I em $\mathrm{G}_{1}^{\alpha}(\mathfrak{U}, \mathfrak{V})$ está contido ${ }^{11}$

\footnotetext{
${ }^{11}$ Não é difícil ver que pela forma como procedemos, o domínio de $\sigma$ é $\left([\bigcup \mathfrak{U}]^{<\omega}\right)^{<\alpha}$. Assim, o domínio de uma estratégia para o jogador I em $\mathrm{G}_{1}^{\alpha}(\mathfrak{U}, \mathfrak{V})$ está em bijeção com um subconjunto do domínio de $\sigma$.
} 
no domínio de $\sigma$ - intuitivamente, se o jogador I sabe como jogar (e vencer) quando o jogador II escolhe finitos elementos por turno, então o jogador I naturalmente sabe como jogar (e vencer) quando o jogador II escolhe apenas um elemento. Relações semelhantes são válidas para o jogador II e para os princípios $S_{1}^{\alpha}(\mathfrak{U}, \mathfrak{V})$ e $S_{\text {fin }}^{\alpha}(\mathfrak{U}, \mathfrak{V})$. Em síntese, temos a seguinte cadeia de implicações:

$$
\begin{aligned}
& \mathrm{II} \uparrow \mathrm{G}_{1}^{\alpha}(\mathfrak{U}, \mathfrak{V}) \quad \Rightarrow \quad \mathrm{I} \not \mathrm{G}_{1}^{\alpha}(\mathfrak{U}, \mathfrak{V}) \quad \Rightarrow \quad \mathrm{S}_{1}^{\alpha}(\mathfrak{U}, \mathfrak{V}) \\
& \Downarrow \quad \Downarrow \quad \Downarrow \\
& \mathrm{II} \uparrow \mathrm{G}_{\text {fin }}^{\alpha}(\mathfrak{U}, \mathfrak{V}) \Rightarrow \mathrm{I} \not \mathrm{G}_{\text {fin }}^{\alpha}(\mathfrak{U}, \mathfrak{V}) \Rightarrow \mathrm{S}_{\text {fin }}^{\alpha}(\mathfrak{U}, \mathfrak{V}) \text {. }
\end{aligned}
$$

Tudo o que fizemos até aqui vale em geral para famílias de conjuntos não vazios $\mathfrak{U}$ e $\mathfrak{V}$, sem a imposição de hipóteses sobre tais famílias, de modo que essencialmente não vimos jogos topológicos propriamente ditos. Assim, a fim de ilustrar a utilização de jogos topológicos, apresentamos a seguir alguns exemplos simples.

Para um espaço topológico $X$, denotamos por $\mathcal{O}(X)$ (ou simplesmente por $\mathcal{O}$, caso não haja risco de ambiguidade) o conjunto de todas as coberturas abertas de $X$. Nos jogos e princípios seletivos que vimos acima, faremos $\mathfrak{U}=\mathfrak{V}=\mathcal{O}$. O jogo de Menger em $X$ é por definição $\mathrm{G}_{f i n}(\mathcal{O}, \mathcal{O})$, e $X$ é dito um espaço de Menger se valer $\mathrm{S}_{\text {fin }}(\mathcal{O}, \mathcal{O})$. Analogamente, o jogo de Rothberger em $X$ é o jogo $\mathrm{G}_{1}(\mathcal{O}, \mathcal{O})$, e $X$ é um espaço de Rothberger se $\mathrm{S}_{1}(\mathcal{O}, \mathcal{O})$.

Proposição 1.4.1. Se X é um espaço enumerável, então X é de Rothberger.

Proposição 1.4.2. Se $X$ é um espaço $\sigma$-compacto, então $\mathrm{II} \uparrow \mathrm{G}_{\text {fin }}(\mathcal{O}, \mathcal{O})$. Em particular, todo espaço $\sigma$-compacto é de Menger.

Demonstração. Seja $X=\bigcup_{n \in \omega} K_{n}$, com cada $K_{n}$ compacto. Fixado $n \in \omega$, para uma cobertura de abertos $\mathcal{U} \in \mathcal{O}$ qualquer, a compacidade de $K_{n}$ nos dá $\mu_{n}(\mathcal{U}) \in[\mathcal{U}]^{<\omega}$ tal que $K_{n} \subseteq$ $\bigcup \mu_{n}(\mathcal{U})$. Assim, basta definirmos a estratégia $\mu$ para o jogador II pondo $\mu\left(\left(\mathcal{U}_{0}, \ldots, \mathcal{U}_{n}\right)\right)=$ $\mu_{n}\left(\mathcal{U}_{n}\right)$, para toda sequência não vazia $\left(\mathcal{U}_{0}, \ldots, \mathcal{U}_{n}\right) \in \mathcal{O}^{<\omega}$.

Proposição 1.4.3. Se X é um espaço de Menger, então X é de Lindelöf.

Demonstração. Se $X$ não for de Lindelöf, então existe uma cobertura aberta $\mathcal{U}$ de $X$ que não admite subcobertura enumerável. Logo, a sequência $\left(\mathcal{U}_{n}\right)_{n \in \omega} \operatorname{com} \mathcal{U}_{n}=\mathcal{U}$ para todo $n \in \omega$ atesta que não vale $S_{f i n}(\mathcal{O}, \mathcal{O})$. 
Em suma, temos as seguintes implicações:

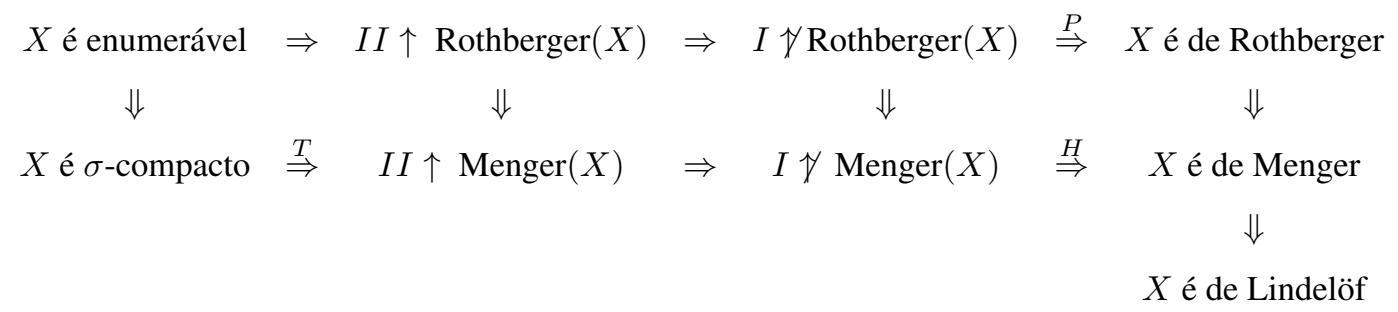

Pawlikowski [36] e Hurewicz [24] provam, respectivamente, as recíprocas de $(\mathrm{P})$ e $(\mathrm{H})$. Em [45], Telgársky exibe um espaço no qual o jogo de Rothberger é indeterminado, e em [44], o autor prova a recíproca de (T), para espaços métricos. Em [16], os autores apresentam subespaços de $\mathbb{R}$ que são de Menger mas não são $\sigma$-compactos, o que mostra que o jogo de Menger também é indeterminado em certos espaços.

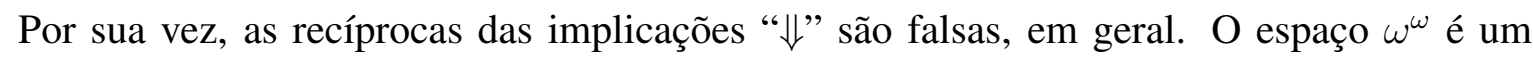
exemplo de espaço de Lindelöf que não é de Menger: basta considerar para cada $n \in \omega$ a cobertura $\mathcal{U}_{m}=\left\{C_{m, n}: m \in \omega\right\}$, onde $C_{m, n}=\left\{f \in \omega^{\omega}: f(m)=n\right\}$, e para qualquer escolha $U_{n} \in\left[\mathcal{U}_{n}\right]^{<\omega}$ obter por um argumento de diagonalização uma função $f \in \omega^{\omega}$ que não pertença a $\bigcup_{n \in \omega} \bigcup U_{n}$. Em particular, isto acarreta que $\omega^{\omega}$ não é $\sigma$-compacto ( $c f$. Seção 4.1).

Por fim, como todo espaço compacto é $\sigma$-compacto, o espaço $2^{\omega}$ atesta a falsidade das recí-

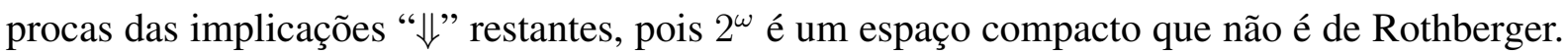
De fato, para cada $n \in \omega$, defina $\mathcal{U}_{n}=\left\{\pi_{n}^{-1}[\{0\}], \pi_{n}^{-1}[\{1\}]\right\}$, onde $\pi_{n}:\{0,1\}^{\omega} \rightarrow\{0,1\}$ é a projeção canônica. Claramente, cada $\mathcal{U}_{n}$ é uma cobertura aberta para $2^{\omega}$. Tome então $V_{n} \in \mathcal{U}_{n}$ para todo $n$ e defina $f: \omega \rightarrow\{0,1\}$ tal que $V_{n} \neq \pi_{n}^{-1}[\{f(n)\}]$ para todo $n$. Enfim, note que $f \in 2^{\omega} \backslash \bigcup_{n \in \omega} V_{n}$. 


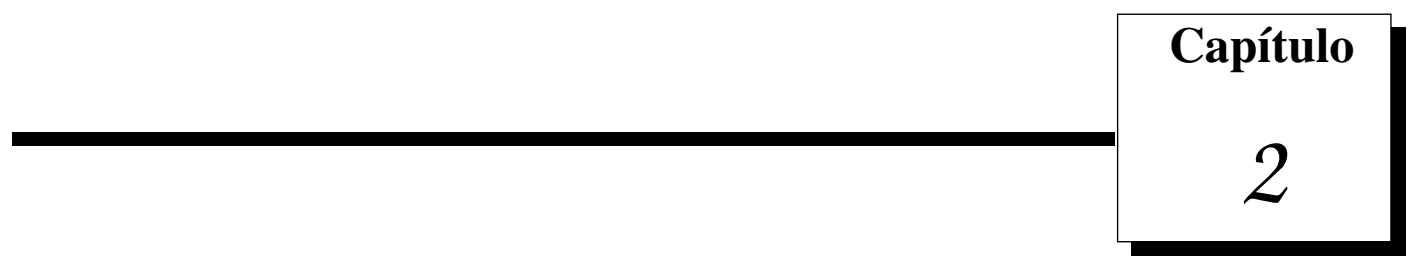

\section{Tópicos de $C_{p}$-Teoria e $C_{k}$-Teoria}

Quando temos um espaço topológico $X$ e por algum motivo precisamos avaliar o conjunto $C(X)$ munido de alguma topologia, digamos $\mathcal{T}_{\mathcal{B}}$, uma pergunta natural que pode surgir é: qual propriedade topológica $\mathcal{P}$ o espaço $X$ precisa ter para garantir que $\left(C(X), \mathcal{T}_{\mathcal{B}}\right)$ tenha a propriedade topológica $\mathcal{Q}$ ? Se respondida, pode ser interessante saber em quais circunstâncias a recíproca é válida. Essa abordagem é uma das principais características do que podemos chamar de " $C_{\mathcal{B}}$-teoria'"1": a teoria dos espaços de funções munidos da topologia $\mathcal{T}_{\mathcal{B}}$. Neste capítulo nos focaremos nos casos em que " $\mathcal{B}=p$ " ou " $\mathcal{B}=k$ ", ou mais precisamente, estudaremos o espaço das funções contínuas munido das topologias produto (da convergência pontual, $\mathcal{T}_{p}$ ) e compacto-aberta $\left(\tau_{k}\right)$.

\subsection{Bornologias}

Nesta seção, definiremos a classe das bornologias sobre um espaço $X$, o que em certo sentido generaliza a noção de limitação. Por meio de tais estruturas, veremos certas variações de propriedades de recobrimento em $X$ e, em termos de uma bornologia $\mathcal{B}$ em $X$, definiremos uma topologia $\mathcal{T}_{\mathcal{B}}$ sobre $C(X)$ que generaliza as topologias compacto-aberta e da convergência pontual quando $X$ é de Hausdorff.

\footnotetext{
1"Estendemos" a definição dada por Tkachuk em [47], onde ele define $C_{p}$-teoria como sendo a teoria dos espaços de funções munidos da topologia da convergência pontual.
} 
Uma bornologia $\mathcal{B}$ sobre um conjunto $X$ é um ideal de subconjuntos não vazios de $X$ que recobre $X$, i.e., $\mathcal{B}$ é uma família de subconjuntos de $X$, hereditária com respeito à inclusão, fechada para reuniões finitas e que satisfaz $X=\cup \mathcal{B}$. Em [22], o autor chama de conjunto bornológico um $\operatorname{par}(X, \mathcal{B})$, onde $\mathcal{B}$ é uma bornologia sobre $X$, e os elementos de $\mathcal{B}$ são chamados de subconjuntos limitados de $X$, o que condiz com os exemplos a seguir.

Num conjunto $X$ desprovido de estruturas, podemos distinguir subconjuntos de $X$ por meio de suas cardinalidades, onde intuitivamente faz sentido definir como limitados os subconjuntos finitos de $X$. É fácil ver que $[X]^{<\omega}$ é uma bornologia em $X$, a bornologia dos conjuntos finitos de $X$, que denotaremos também por $\mathcal{F}_{X}$, ou simplesmente por $\mathcal{F}$ quando o conjunto $X$ estiver claro pelo contexto.

Quando $X$ está munido de uma topologia $\tau$, também podemos considerar outra noção de limitação, na qual dizemos que um subconjunto de $X$ é limitado se estiver contido num subespaço compacto de $X$. Isso define uma bornologia no espaço $X$, já que os subconjuntos finitos de $X$ são compactos e a reunião finita de compactos é compacta; tal bornologia será denotada por $\mathfrak{K}_{X}$ ou $\mathfrak{K}$. Em particular, quando $X$ é de Hausdorff temos

$$
\mathfrak{K}_{X}=\{A \subseteq X: \bar{A} \text { é compacto }\}
$$

Nos casos em que a bornologia $\mathcal{B}$ sobre $X$ coincide com $\wp(X)$, chamamos $\mathcal{B}$ de bornologia trivial. É o que ocorre com $\mathfrak{K}_{X}$ (ou $\mathcal{F}_{X}$ ) se $X$ é compacto (ou finito, respectivamente). Há outros exemplos de bornologias sobre diversos tipos de espaços; ao leitor interessado recomendamos o livro de Hogbe-Nlend [22], que faz uma introdução à noção de bornologias voltada para a Análise Funcional. Para o nosso trabalho, já definimos todas as bornologias que usaremos nos casos particulares.

Uma base para a bornologia $\mathcal{B}$ é um subconjunto $\mathcal{B}_{0} \subseteq \mathcal{B}$ tal que para todo $B \in \mathcal{B}$ existe $B_{0} \in \mathcal{B}_{0} \operatorname{com} B \subseteq B_{0}$. Note que se $\mathcal{A} \subseteq \wp(X)$ recobrir $X$ e, para quaisquer $A_{0}, A_{1} \in \mathcal{A}$ existir $A_{2} \in \mathcal{A}$ satisfazendo $A_{0} \cup A_{1} \subseteq A_{2}$, então a família

$$
\mathcal{B}(\mathcal{A}):=\{B \in \wp(X) \backslash\{\emptyset\}: \exists A \in \mathcal{A}(B \subseteq A)\}
$$


é claramente uma bornologia em $X$, que tem $\mathcal{A}$ como base por construção. Mais geralmente, se $\mathcal{A}$ apenas recobrir $X$, então $(\mathcal{A})_{\text {fin }}:=\left\{\bigcup F: F \in[\mathcal{A}]^{<\omega}\right\}$ também é base para uma bornologia, que denotaremos igualmente por $\mathcal{B}(\mathcal{A})$, embora neste caso optemos por dizer que $\mathcal{A}$ gera a bornologia como sub-base.

Quando $X$ é um espaço topológico e $\mathcal{B}$ é uma bornologia sobre $X$, dizemos que $\mathcal{B}$ tem base compacta (fechada, etc.) se existe uma base $\mathcal{B}_{0}$ para $\mathcal{B}$ cujos elementos são compactos (fechados, etc.) na topologia de $X$. Ambas as bornologias $\mathfrak{K}_{X}$ e $\mathcal{F}_{X}$ têm bases compactas no espaço $X$; note que se $\mathcal{B}$ tem base compacta, então $\mathcal{F}_{X} \subseteq \mathcal{B} \subseteq \mathfrak{K}_{X}$. Se $\mathcal{B}$ é uma bornologia sobre $X$, então $\{\bar{B}: B \in \mathcal{B}\}$ é base fechada para uma bornologia sobre $X$, que denotamos por $\overline{\mathcal{B}}$; claramente temos $\mathcal{B} \subseteq \overline{\mathcal{B}}$. Além disso, é fácil ver que as seguintes condições são equivalentes:

(i) a bornologia $\mathcal{B}$ tem base fechada;

(ii) $\bar{B} \in \mathcal{B}$ para todo $B \in \mathcal{B}$;

(iii) $\mathcal{B}=\overline{\mathcal{B}}$.

Consideremos agora uma família $\left\{\left(X_{i}, \tau_{i}\right): i \in I\right\}$ de espaços topológicos e uma coleção $\left\{\mathcal{A}_{i}: i \in I\right\} \operatorname{com} A_{i} \subseteq \wp\left(X_{i}\right)$ para cada $i$. Se cada $\mathcal{A}_{i}$ for uma base (compacta) para uma borno$\operatorname{logia} \mathcal{B}_{i}$ sobre $X_{i}$, então podemos definir bornologias (com bases compactas, respectivamente) sobre $\prod_{i \in I} X_{i}$ e $\sum_{i \in I} X_{i}$ de uma maneira natural.

Em $X=\prod_{i \in I} X_{i}$, considere a família $\mathcal{A}$ dos conjuntos da forma $\prod_{i \in I} A_{i}$ para $A_{i} \in \mathcal{A}_{i}$. Como cada $\mathcal{A}_{i}$ é uma base para a bornologia $\mathcal{B}_{i}$ sobre $X_{i}$, segue que $\mathcal{A}$ é uma base para uma bornologia sobre $X$, que chamamos de bornologia produto e denotamos por $\bigotimes_{i \in I} \mathcal{B}_{i}$. Pelo Teorema de Tychonoff é fácil ver que a base $\mathcal{A}$ é compacta se, e somente se, cada $\mathcal{A}_{i}$ é uma base compacta. Em particular, frisamos que

$$
\bigotimes_{i \in I} \mathcal{F}_{X_{i}}=\mathcal{F}_{X} \text { e } \bigotimes_{i \in I} \mathfrak{K}_{X_{i}}=\mathfrak{K}_{X}
$$

Por sua vez, em $Y=\sum_{i \in I} X_{i}$ vamos definir a coleção $\mathcal{A}^{\prime}$ de todos os conjuntos da forma $\bigsqcup_{i \in I} A_{i}$, onde $0<\left|\left\{i \in I: A_{i} \neq \emptyset\right\}\right|<\aleph_{0}$ e $A_{i} \in \mathcal{A}_{i}$ se $A_{i} \neq \emptyset$. A hipótese de que cada $\mathcal{A}_{i}$ é uma base para a bornologia $\mathcal{B}_{i}$ sobre $X_{i}$ garante que $\mathcal{A}^{\prime}$ é base para uma bornologia sobre $Y$, 
que chamamos de bornologia soma (ou soma das bornologias) e simbolizamos por $\bigoplus_{i \in I} \mathcal{B}_{i}$. Como $K \subseteq \sum_{i \in I} X_{i}$ é compacto se, e somente se, $K=\bigsqcup_{i \in I} K_{i}$, com cada $K_{i} \subseteq X_{i}$ compacto e $\left|\left\{i \in I: K_{i} \neq \emptyset\right\}\right|<\aleph_{0}$, segue que $\bigoplus_{i \in I} \mathcal{B}_{i}$ tem base compacta se, e somente se, cada $\mathcal{B}_{i}$ tem base compacta. Analogamente a observação 2.1.3 feita acima, note que

$$
\bigoplus_{i \in I} \mathcal{F}_{X_{i}}=\mathcal{F}_{Y} \text { e } \bigoplus_{i \in I} \mathfrak{K}_{X_{i}}=\mathfrak{K}_{Y}
$$

Em particular, se $\mathcal{B}$ é uma bornologia sobre $X$ e $I=m \in \omega$, chamamos $\mathcal{B}^{m}:=\bigotimes_{i \in I} \mathcal{B}$ e $m \mathcal{B}:=\bigoplus_{i \in I} \mathcal{B}$

\subsubsection{Bornologias e propriedades de recobrimento}

Nesta subseção, utilizamos bornologias para estudar certas propriedades de recobrimento num espaço topológico. Tais resultados terão importância nas seções seguintes, em que propriedades de convergência em $C(X)$ serão relacionadas com propriedades de recobrimento em $X$. Ao longo desta subseção, vamos considerar $X$ um espaço topológico munido de uma bornologia $\mathcal{B}$.

Adaptando a definição de Caserta et al. [9, 10], uma família $\mathcal{U}$ de abertos de $X$ será chamada de $\mathcal{B}$-cobertura aberta para $X$ (ou simplesmente $\mathcal{B}$-cobertura para $X$ ) se para todo $B \in \mathcal{B}$ existir $U \in \mathcal{U}$ tal que $B \subseteq U$. Em particular, é usual na literatura chamar uma $\mathcal{F}$-cobertura de $\omega$-cobertura e uma $\mathfrak{K}$-cobertura de $K$-cobertura.

Se tivermos $X \in \mathcal{U}$, então $\mathcal{U}$ é trivialmente uma $\mathcal{B}$-cobertura e, por tal motivo, $\mathcal{B}$-coberturas que contiverem o espaço $X$ como elemento serão chamadas de coberturas triviais. Note que se $\mathcal{B}$ é a bornologia trivial, então toda $\mathcal{B}$-cobertura é necessariamente trivial.

Denotamos por $\mathcal{O}_{\mathcal{B}}(X)$ a família de todas as $\mathcal{B}$-coberturas de $X$ e por $\mathcal{O}_{\mathcal{B}}(X)^{*}$ a coleção de todas as $\mathcal{B}$-coberturas de $X$ não triviais - quando o espaço $X$ estiver claro pelo contexto, escreveremos simplesmente $\mathcal{O}_{\mathcal{B}}$ e $\mathcal{O}_{\mathcal{B}}^{*}$. Em particular, a coleção de todas as $\omega$-coberturas de $X$ será denotada por $\Omega(X)$ (ou $\Omega$ ), e a coleção de todas as $K$-coberturas de $X$ será denotada por $\mathcal{K}(X)$ (ou $\mathcal{K}$ ); além disso, segue da observação 2.1.3) que $\mathcal{O}_{\mathcal{F}^{m}}=\Omega\left(X^{m}\right)$ e $\mathcal{O}_{\mathfrak{K}^{m}}=\mathcal{K}\left(X^{m}\right)$.

Os elementos de uma $\mathcal{B}$-cobertura não trivial $\mathcal{U}$ devem ser "gradativamente" grandes, já que podemos obter elementos de $\mathcal{B}$ cada vez "maiores" por meio de reuniões finitas, os quais devem 
estar cobertos pelos abertos de $\mathcal{U}$. Assim, para um $B \in \mathcal{B}$ coberto por algum $U \in \mathcal{U}$, existe pelo menos um ponto $x$ de $X$ que não é recoberto por $U$ (pois $U \neq X$ ) e, consequentemente, $B^{\prime}=B \cup\{x\}$ é um membro de $\mathcal{B}$ que não é recoberto por $U$. Logo, deve existir $U^{\prime} \in \mathcal{U}$ que recobre $B^{\prime}$. Procedendo dessa maneira indutivamente, prova-se o seguinte lema.

Lema 2.1.1. Se $\mathcal{U} \in \mathcal{O}_{\mathcal{B}}$ é não trivial, então para qualquer $F \in[\mathcal{U}]^{<\omega}$ temos $\mathcal{U} \backslash F \in \mathcal{O}_{\mathcal{B}}$.

Por meio das $\mathcal{B}$-coberturas estudaremos algumas variações de propriedades de recobrimento em $X$. Primeiramente, apresentamos uma generalização natural da propriedade "ser de Lindelöf".

O grau de Lindelöf é a função cardinal que associa a um espaço topológico $X$ o cardinal

$$
l(X):=\min \left\{\kappa: \forall \mathcal{U} \in \mathcal{O}(X)\left(\exists \mathcal{U}^{\prime} \in[\mathcal{U}]^{\leq \kappa} \cap \mathcal{O}(X)\right)\right\}+\aleph_{0}
$$

Na definição acima, trocando "coberturas" por “ $\mathcal{B}$-coberturas", obtemos nossa primeira variação. O grau de $\mathcal{B}$-Lindelöf, denotado por $l_{\mathcal{B}}(X)$, é o menor cardinal transfinito $\kappa$ tal que para toda $\mathcal{B}$-cobertura para $X$ existe uma $\mathcal{B}$-cobertura de cardinalidade $\leq \kappa$, em símbolos:

$$
l_{\mathcal{B}}(X):=\min \left\{\kappa: \forall \mathcal{U} \in \mathcal{O}_{\mathcal{B}}(X)\left(\exists \mathcal{U}^{\prime} \in[\mathcal{U}]^{\leq \kappa} \cap \mathcal{O}_{\mathcal{B}}(X)\right)\right\}+\aleph_{0}
$$

É fácil ver que $X$ é um espaço de Lindelöf se, e somente se, $l(X)=\aleph_{0}$. Por analogia, diremos também que $X$ é um espaço $\mathcal{B}$-Lindelöf se $l_{\mathcal{B}}(X)=\aleph_{0}$. Na proposição seguinte, agrupamos as principais observações sobre os graus de Lindelöf e de $\mathcal{B}$-Lindelöf.

\section{Proposição 2.1.2.}

(a) Se $\mathcal{B}$ tem base de Lindelöf, então $l(X) \leq l_{\mathcal{B}}(X)$.

(b) (Adaptado de Kočinac, [14], Lema 3) Se $\mathcal{B}$ tem base compacta, $m \in \omega$ e $\mathcal{U}$ é uma $\mathcal{B}^{m}$ cobertura em $X^{m}$, então existe uma $\mathcal{B}$-cobertura aberta $\mathcal{V}$ de $X$ tal que a família $\mathcal{V}^{m}:=$ $\left\{V^{m}: V \in \mathcal{V}\right\}$ é uma $\mathcal{B}^{m}$-cobertura de $X^{m}$ que refina $\mathcal{U}$.

(c) Se $\mathcal{B}$ tem base compacta, então $l_{\mathcal{B}}(X)=\sup \left\{l_{\mathcal{B}^{m}}\left(X^{m}\right): m \in \omega\right\}$. Em particular, temos também $\sup \left\{l\left(X^{n}\right): n \in \omega\right\} \leq l_{\mathcal{B}}(X)$. 


\section{Demonstração.}

(a). Sejam $\mathcal{B}_{0}$ uma base de Lindelöf para $\mathcal{B}, \mathcal{U}$ uma cobertura de abertos para $X$ e digamos que $l_{\mathcal{B}}(X)=\kappa$. Para cada $B \in \mathcal{B}_{0}$, existe uma coleção enumerável $\mathcal{V}_{B} \subseteq \mathcal{U}$ tal que $B \subseteq \bigcup \mathcal{V}_{B}$, pois $B$ é de Lindelöf. Assim, $\mathcal{V}=\left\{\bigcup \mathcal{V}_{B}\right\}_{B \in \mathcal{B}_{0}}$ é uma $\mathcal{B}$-cobertura para $X$ e, pela hipótese, existe $\left\{B_{\alpha}: \alpha<\kappa\right\} \subseteq \mathcal{B}_{0}$ tal que $\left\{\bigcup \mathcal{V}_{B_{\alpha}}\right\}_{\alpha<\kappa}$ é uma $\mathcal{B}$-subcobertura de $X$. Enfim, como cada $\mathcal{V}_{B_{\alpha}}$ é enumerável, segue que $\bigcup\left\{\mathcal{V}_{B_{\alpha}}: \alpha<\kappa\right\} \subseteq \mathcal{U}$ é uma subcobertura de cardinalidade $\leq \kappa$.

(b). A demonstração consiste numa aplicação conveniente do Teorema de Wallace. Para $m>1$ e $\bar{B} \in \mathcal{B}$ compacto, existem $U \in \mathcal{U}$ e abertos $V_{0, B}, \ldots, V_{m-1, B} \subseteq X$ satisfazendo

$$
\bar{B}^{m} \subseteq \prod_{j<m} V_{j, B} \subseteq U
$$

Afirmamos que $\mathcal{V}=\left\{V_{B}: B \in \mathcal{B}\right\}$ tem as propriedades desejadas, onde $V_{B}=\bigcap_{j<m} V_{j, B}$. Claramente $\mathcal{V}$ é uma $\mathcal{B}$-cobertura para $X$, e $\mathcal{V}^{m}$ refina $\mathcal{U}$ por construção; resta apenas mostrarmos que $\mathcal{V}^{m}$ é uma $\mathcal{B}^{m}$-cobertura para $X^{m}$. Ora, para $A \in \mathcal{B}^{m}$, existem $B_{0}, \ldots, B_{m-1} \in \mathcal{B}$ satisfazendo $A \subseteq \prod_{i \leq m} B_{i}$ e, por conseguinte, $A \subseteq \prod_{i<m} B$, onde $B=\bigcup_{i<m} B_{i} \in \mathcal{B}$. Logo, existe $V \in \mathcal{V}$ tal que $B \subseteq V$ e, consequentemente, $A \subseteq V^{m}$, mostrando que $\mathcal{V}^{m} \in \mathcal{O}_{\mathcal{B}^{m}}$.

$(c)$. Fixe $m \in \omega$, suponha $l_{\mathcal{B}}(X) \leq \kappa$ e considere $\mathcal{U}$ uma $\mathcal{B}^{m}$-cobertura de $X^{m}$. Pelo item (b) acima, existe uma $\mathcal{B}$-cobertura $\mathcal{V}$ de $X$ tal que $\left\{V^{m}: V \in \mathcal{V}\right\}$ é uma $\mathcal{B}^{m}$-cobertura de $X^{m}$ que refina $\mathcal{U}$. Pela hipótese, existe $\mathcal{V}^{\prime}=\left\{V_{\alpha}: \alpha<\kappa\right\} \subseteq \mathcal{V}$ uma $\mathcal{B}$-subcobertura de $X$ e, como na demonstração do item anterior, mostra-se que $\left\{V_{\alpha}^{m}: \alpha<\kappa\right\}$ é uma $\mathcal{B}^{m}$-cobertura para $X^{m}$. Tomando $\mathcal{U}^{\prime}=\left\{U_{\alpha}: \alpha<\kappa\right\} \subseteq \mathcal{U}$ de modo que $U_{\alpha} \in \mathcal{U}$ satisfaz $V_{\alpha}^{m} \subseteq U_{\alpha}$ para cada $\alpha<\kappa$, segue que $\mathcal{U}^{\prime}$ é uma $\mathcal{B}^{m}$-subcobertura para $X^{m} . \operatorname{Logo}, \sup \left\{l_{\mathcal{B}^{m}}\left(X^{m}\right): m \in \omega\right\} \leq$ $l_{\mathcal{B}}(X)$, enquanto a outra desigualdade é válida por definição. Por fim, como neste caso temos $l_{\mathcal{B}^{m}}\left(X^{m}\right) \leq l_{\mathcal{B}}(X)$ para todo $m$, a desigualdade $\sup \left\{l\left(X^{n}\right): n \in \omega\right\} \leq l_{\mathcal{B}}(X)$ segue do item $(a)$.

Quando $\mathcal{B}=\mathcal{F}$, a desigualdade do item $(c)$ da proposição anterior é na verdade uma igualdade.

Proposição 2.1.3 (Gerlits e Nagy, [18], Teorema 2). Para um espaço topológico X, temos $l_{\mathcal{F}}(X)=\sup \left\{l\left(X^{n}\right): n \in \omega\right\}$. 
Demonstração. (Prova adaptada de Tkachuk, [47], Problema 123). Como $\mathcal{F}$ tem base compacta, o item $(c)$ da proposição anterior nos dá $\sup \left\{l\left(X^{n}\right): n \in \omega\right\} \leq l_{\mathcal{F}}(X)$. Provemos a outra desigualdade.

Chame $\kappa=\sup \left\{l\left(X^{m}\right): m \in \omega\right\}$, e tome $\mathcal{U}$ uma $\omega$-cobertura para $X$. Para qualquer $m \in \omega, \mathcal{U}^{m}:=\left\{U^{m}: U \in \mathcal{U}\right\}$ é uma cobertura de $X^{m}:$ de fato, dado $\left(x_{0}, \ldots, x_{m-1}\right) \in X^{m}$, temos $F=\left\{x_{0}, \ldots, x_{m-1}\right\} \subset X$ finito, donde segue que existe $U \in \mathcal{U}$ com $F \subseteq U$ e, por conseguinte, $\left(x_{0}, \ldots, x_{m-1}\right) \in U^{m} \in \mathcal{U}^{m}$. Logo, existe $\mathcal{V}^{m} \in[\mathcal{U}]^{\leq \kappa}$ tal que $\left\{V^{m}: V \in \mathcal{V}^{m}\right\}$ é cobertura aberta para $X^{m}$. A família $\mathcal{U}^{\prime}=\bigcup\left\{\mathcal{V}^{m}: m \in \omega\right\} \subseteq \mathcal{U}$ tem claramente cardinalidade $\leq \kappa$. Assim, basta notar que $\mathcal{U}^{\prime} \in \Omega$.

No capítulo anterior, na Seção 1.4. definimos propriedades de recobrimento por meio dos princípios de seleção $\mathrm{S}_{1}$ e $\mathrm{S}_{f i n}$ e da família $\mathcal{O}$ das coberturas abertas de $X$. Agora, da mesma forma como trocamos os termos "cobertura" por " $\mathcal{B}$-cobertura" para definirmos o grau de $\mathcal{B}$ Lindelöf, vamos utilizar a família $\mathcal{O}_{\mathcal{B}}$ no lugar da família $\mathcal{O}$, e estudaremos os princípios de seleção $\mathrm{S}_{1}\left(\mathcal{O}_{\mathcal{B}}, \mathcal{O}_{\mathcal{B}}\right)$ e $\mathrm{S}_{\text {fin }}\left(\mathcal{O}_{\mathcal{B}}, \mathcal{O}_{\mathcal{B}}\right)$.

Primeiramente, observamos que assim como $\mathrm{S}_{f i n}(\mathcal{O}, \mathcal{O})$ implica em $l(X)=\aleph_{0}$, a hipótese $\mathrm{S}_{\text {fin }}\left(\mathcal{O}_{\mathcal{B}}, \mathcal{O}_{\mathcal{B}}\right)$ garante que $l_{\mathcal{B}}(X)=\aleph_{0}$. Além disso, se $\mathcal{B}$ tem base compacta, então

$$
\mathrm{S}_{\text {fin }}\left(\mathcal{O}_{\mathcal{B}}, \mathcal{O}_{\mathcal{B}}\right) \Rightarrow \mathrm{S}_{f i n}(\mathcal{O}, \mathcal{O})
$$

a prova é análoga à demonstração do item $(a)$ da Proposição 2.1.2. Mais geralmente, temos:

Teorema 2.1.4 (Adaptado de Kočinac, [14], Teoremas 5 e 6). Suponha que $\mathcal{B}$ tenha base compacta e seja $\alpha \geq \omega$ um ordinal.

(a) X satisfaz $\mathrm{S}_{\text {fin }}^{\alpha}\left(\mathcal{O}_{\mathcal{B}}, \mathcal{O}_{\mathcal{B}}\right)$ se, e somente se, $X^{m}$ satisfaz $\mathrm{S}_{\text {fin }}^{\alpha}\left(\mathcal{O}_{\mathcal{B}^{m}}, \mathcal{O}_{\mathcal{B}^{m}}\right)$ para todo $m \in \omega$.

(b) X satisfaz $\mathrm{S}_{1}^{\alpha}\left(\mathcal{O}_{\mathcal{B}}, \mathcal{O}_{\mathcal{B}}\right)$ se, e somente se, $X^{m}$ satisfaz $\mathrm{S}_{1}^{\alpha}\left(\mathcal{O}_{\mathcal{B}^{m}}, \mathcal{O}_{\mathcal{B}^{m}}\right)$ para todo $m \in \omega$.

Demonstração. Como as demonstrações de $(a)$ e $(b)$ são semelhantes, provaremos apenas $(b)$.

Suponha $\mathrm{S}_{1}^{\alpha}\left(\mathcal{O}_{\mathcal{B}}, \mathcal{O}_{\mathcal{B}}\right)$ e seja $\left(\mathcal{W}_{\gamma}\right)_{\gamma<\alpha}$ uma sequência de $\mathcal{B}^{m}$-coberturas de $X^{m}$. Pelo item (b) do Lema 2.1.2, obtemos uma sequência $\left(\mathcal{U}_{\gamma}\right)_{\gamma<\alpha}$ de $\mathcal{B}$-coberturas para $X$ tais que $\left\{U^{m}\right.$ : $\left.U \in \mathcal{U}_{\gamma}\right\}$ refina $\mathcal{W}_{\gamma}$ para cada $\gamma<\alpha$. Pela hipótese, existe $\left(U_{\gamma}\right)_{\gamma<\alpha}$, com $U_{\gamma} \in \mathcal{U}_{\gamma}$, tal que $\mathcal{U}=\left\{U_{\gamma}: \gamma<\alpha\right\}$ é uma $\mathcal{B}$-cobertura para $X$. Enfim, para cada $\gamma$ escolha $W_{\gamma} \in \mathcal{W}_{\gamma}$ tal que 
$U_{\gamma}^{m} \subseteq W_{\gamma}$ e note que $\left\{U_{\gamma}^{m}: \gamma<\alpha\right\}$ é uma $\mathcal{B}^{m}$-cobertura que refina $\mathcal{W}=\left\{W_{\gamma}: \gamma<\alpha\right\}$. Logo, $\mathcal{W} \in \mathcal{O}_{\mathcal{B}^{m}}$.

Para $\mathcal{B}=\mathfrak{K}$ e $\alpha=\omega$, obtemos os resultados que motivaram o teorema anterior.

Corolário 2.1.5 (Kočinac, [14], Teoremas 5 e 6). Seja X um espaço topológico.

(a) $\mathrm{S}_{1}(\mathcal{K}(X), \mathcal{K}(X))$ se, e somente se, $\mathrm{S}_{1}\left(\mathcal{K}\left(X^{m}\right), \mathcal{K}\left(X^{m}\right)\right)$ para todo $m \in \omega$.

(b) $\mathrm{S}_{\text {fin }}(\mathcal{K}(X), \mathcal{K}(X))$ se, e somente se, $\mathrm{S}_{\text {fin }}\left(\mathcal{K}\left(X^{m}\right), \mathcal{K}\left(X^{m}\right)\right)$ para todo $m \in \omega$.

O teorema anterior também melhora a conclusão obtida em 2.1.7): como $\mathrm{S}_{\text {fin }}\left(\mathcal{O}_{\mathcal{B}}, \mathcal{O}_{\mathcal{B}}\right)$ é equivalente a $\mathrm{S}_{\text {fin }}\left(\mathcal{O}_{\mathcal{B}^{m}}, \mathcal{O}_{\mathcal{B}^{m}}\right)$ para todo $m \in \omega$, aplicando 2.1.7) obtemos

$$
\mathrm{S}_{\text {fin }}\left(\mathcal{O}_{\mathcal{B}}, \mathcal{O}_{\mathcal{B}}\right) \Rightarrow \forall m \in \omega\left(\mathrm{S}_{\text {fin }}\left(\mathcal{O}\left(X^{m}\right), \mathcal{O}\left(X^{m}\right)\right)\right)
$$

cuja recíproca é verdadeira quando $\mathcal{B}=\mathcal{F}$.

\section{Proposição 2.1.6.}

(a) (Scheepers et al., [26], Teorema 3.9) X satisfaz $\mathrm{S}_{f i n}(\Omega, \Omega)$ se, e somente se, $X^{m}$ é de Menger para todo $m \in \omega$.

(b) (Sakai, [38], Lema 2) X satisfaz $\mathrm{S}_{1}(\Omega, \Omega)$ se, e somente se, $X^{m}$ é de Rothberger para todo $m \in \omega$.

Demonstração. (a). Se $X$ satisfaz $\mathrm{S}_{f i n}(\Omega, \Omega)$, então pela observação anterior segue que $X^{m}$ satisfaz $\mathrm{S}_{f i n}\left(\mathcal{O}\left(X^{m}\right), \mathcal{O}\left(X^{m}\right)\right)$. Reciprocamente, assuma que $X^{m}$ satisfaz $\mathrm{S}_{\text {fin }}(\mathcal{O}, \mathcal{O})$ para todo $m$ e tome uma sequência $\left(\mathcal{U}_{n}\right)_{n \in \omega}$ de $\omega$-coberturas de $X$, que reescrevemos como $\left(\mathcal{U}_{m, n}\right)_{m, n \in \omega}$. Para cada $m, n \in \omega$, temos $\mathcal{V}_{m, n}=\left\{U^{m}: U \in \mathcal{U}_{m, n}\right\}$ uma cobertura para $X^{m}$ e, assim, $\left(\mathcal{V}_{m, n}\right)_{n \in \omega}$ é uma sequência de coberturas para $X^{m}$. A hipótese então nos dá $F_{m, n} \in\left[\mathcal{U}_{m, n}\right]^{<\omega}$ para cada $m, n \in \omega$, tal que $\left\{U^{m}: U \in F_{m, n}, n \in \omega\right\}$ é uma cobertura para $X^{m}$. Então $\bigcup_{m, n \in \omega} F_{m, n}$ é claramente uma $\omega$-cobertura para $X$.

(b). $(\Rightarrow)$ Em vista do Teorema anterior, é suficiente mostrarmos que $S_{1}(\Omega, \Omega)$ implica $\mathrm{S}_{1}(\mathcal{O}, \mathcal{O})$, pois $\mathrm{S}_{1}(\Omega(X), \Omega(X))$ é equivalente a $\mathrm{S}_{1}\left(\Omega\left(X^{m}\right), \Omega\left(X^{m}\right)\right)$. Suponha que $X$ satisfaz $\mathrm{S}_{1}(\Omega, \Omega)$ e considere $\left(\mathcal{U}_{n}\right)_{n \in \omega}$ uma sequência de coberturas de $X$, a qual reenumeramos como 
$\left(\mathcal{U}_{m, n}\right)_{m, n \in \omega}$. Para $m \in \omega$ fixado, chame $\mathcal{V}_{m, 0}=\mathcal{U}_{m, 0}$ e, para $n \geq 1, \mathcal{V}_{m, n}=\left\{U_{0} \cup \cdots \cup U_{n-1}\right.$ : $\left.\forall i<n\left(U_{i} \in \mathcal{U}_{m, n+i}\right)\right\}$; claramente a família $\mathcal{V}_{m}=\bigcup_{n \in \omega} \mathcal{V}_{m, n}$ é uma $\omega$-cobertura para $X$. A hipótese então nos dá $V_{m} \in \mathcal{V}_{m}$ para cada $m$, de modo que $\left\{V_{m}: m \in \omega\right\}$ é uma $\omega$-cobertura de $X$, donde segue que $X$ satisfaz $\mathrm{S}_{1}(\mathcal{O}, \mathcal{O})$.

$(\Leftarrow)$. Análoga à demonstração do item anterior.

\subsubsection{Topologia da convergência uniforme numa bornologia}

O último tópico desta seção trata de uma topologia sobre um espaço de funções definida em termos de uma bornologia. Ao longo desta subseção, a menos que expresso o contrário, $X, Y$ e $\mathcal{B}$ denotam, respectivamente, um espaço topológico $X$, um espaço uniforme $Y$ munido de uma uniformidade $\mathfrak{U}$ e uma bornologia $\mathcal{B}$ sobre $X$.

Vamos considerar conjuntos da forma

$$
\langle B, \mathcal{U}\rangle:=\left\{(f, g) \in Y^{X} \times Y^{X}: \forall x \in B((f(x), g(x)) \in \mathcal{U})\right\}
$$

com $B \in \mathcal{B}$ e $\mathcal{U} \in \mathfrak{U}$ (compare com os conjuntos definidos em (1.3.3)). Note que a família composta por todos os conjuntos da forma $\langle B, \mathcal{U}\rangle$ satisfaz as condições (BU11)-(BU4) da definição de base para uma uniformidade:

(BU1) como $\Delta(Y) \subseteq \mathcal{U}$, segue que $\Delta\left(Y^{X}\right) \subseteq\langle B, \mathcal{U}\rangle$ para quaisquer $B \in \mathcal{B}$ e $\mathcal{U} \in \mathfrak{U}$;

(BU2) se $B_{0}, B_{1} \in \mathcal{B}$ e $\mathcal{U}, \mathcal{V} \in \mathfrak{U}$, então $\left\langle B_{0} \cup B_{1}, \mathcal{U} \cap \mathcal{V}\right\rangle \subseteq\left\langle B_{0}, \mathcal{U}\right\rangle \cap\left\langle B_{1}, \mathcal{V}\right\rangle$, pois $B_{0} \cup B_{1} \in \mathcal{B}$ e $\mathcal{U} \cap \mathcal{V} \in \mathfrak{U}$

(BU3) se $\mathcal{U} \in \mathfrak{U}$, então existe $\mathcal{V} \in \mathfrak{U} \operatorname{com} \mathcal{V}^{-1} \subseteq \mathcal{U}$, e assim $\langle B, \mathcal{V}\rangle$ é tal que $(\langle B, \mathcal{V}\rangle)^{-1} \subseteq$ $\langle B, \mathcal{U}\rangle$, para qualquer $B \in \mathcal{B}$;

(BU4) como para cada $\mathcal{U} \in \mathfrak{U}$ existe $\mathcal{V} \in \mathfrak{U}$ que satisfaz $\mathcal{V} \circ \mathcal{V} \subseteq \mathcal{U}$, temos $\langle B, \mathcal{V}\rangle \circ\langle B, \mathcal{V}\rangle \subseteq$ $\langle B, \mathcal{U}\rangle$, para todo $B \in \mathcal{B}$.

Vamos chamar de $\mathfrak{U}(\mathcal{B})$ a uniformidade sobre $Y^{X}$ gerada pelos conjuntos da forma $\langle B, \mathcal{U}\rangle$. Se $\bigcap \mathfrak{U}=\Delta(Y)$, então $\bigcap \mathfrak{U}(\mathcal{B})=\Delta\left(Y^{X}\right)$, donde segue que $Y^{X}$ é um espaço de Tychonoff 
munido da topologia $\mathcal{T}_{\mathcal{B}}:=\tau\left(Y^{X}, \mathfrak{U}(\mathcal{B})\right)$ sempre que $(Y, \tau(Y, \mathfrak{U}))$ é de Tychonoff ( $c f$. Proposição 1.3.3, item (b)). Para uma net $\left(f_{l}\right)_{l \in L}$ em $Y^{X}$ e $f \in Y^{X}, f_{l} \rightarrow \mathcal{T}_{\mathcal{B}} f$ se, e somente se, $f_{l} \uparrow B \rightarrow f \uparrow B$ uniformemente para todo $B \in \mathcal{B}$. De fato, como uma vizinhança básica de $f \in Y^{X}$ é da forma $\langle B, \mathcal{U}\rangle[f]=\left\{g \in Y^{X}:(f, g) \in\langle B, \mathcal{U}\rangle\right\}$, temos

$$
\begin{gathered}
f_{l} \rightarrow \mathcal{T}_{\mathcal{B}} f \Leftrightarrow(\forall B \in \mathcal{B})(\forall \mathcal{U} \in \mathfrak{U})\left(\exists l_{0} \in L\left(l \geq l_{0} \Rightarrow f_{l} \in\langle B, \mathcal{U}\rangle[f]\right)\right) \Leftrightarrow \\
\Leftrightarrow(\forall B \in \mathcal{B})(\forall \mathcal{U} \in \mathfrak{U})\left(\exists l_{0} \in L\left(l \geq l_{0} \Rightarrow\left(\forall x \in B\left(\left(f(x), f_{l}(x)\right) \in \mathcal{U}\right)\right)\right) \Leftrightarrow\right. \\
\Leftrightarrow(\forall B \in \mathcal{B})\left(f_{l} \uparrow B \rightarrow f \uparrow B \text { uniformemente }\right) .
\end{gathered}
$$

Por tal motivo, a topologia $\mathcal{T}_{\mathcal{B}}$ será chamada de topologia da convergência uniforme em B. Denotaremos por $C_{\mathcal{B}}(X, Y)$ o conjunto $C(X, Y)$ munido da topologia de subespaço de $\left(Y^{X}, \mathcal{T}_{\mathcal{B}}\right)$, e $C_{\mathcal{B}}(X):=C_{\mathcal{B}}(X, \mathbb{R})$.

É fácil ver que $\mathcal{T}_{p}$ (a topologia produto) coincide com $\mathcal{T}_{\mathcal{F}}$ (a topologia da convergência uniforme em $\mathcal{F}$ ) em $Y^{X}$ quando $Y$ é um espaço uniforme, o que acarreta $C_{p}(X, Y)=C_{\mathcal{F}}(X, Y)$ em tal caso. Da mesma forma, se $X$ é de Hausdorff, então $\mathcal{T}_{k}$ (a topologia da convergência uniforme em compactos) é igual a $\mathcal{T}_{\mathfrak{K}}$ (a topologia da convergência uniforme em $\mathfrak{K}$ ). Mais ainda, se $\mathcal{B}$ for a bornologia trivial, temos $X \in \mathcal{B}$ e assim é suficiente considerarmos as entourages da forma

$$
\langle\mathcal{U}\rangle:=\langle X, \mathcal{U}\rangle
$$

pois $\langle X, \mathcal{U}\rangle \subseteq\langle B, \mathcal{U}\rangle$ para quaisquer $B \in \mathcal{B}$ e $\mathcal{U} \in \mathfrak{U}$ (compare com os conjuntos da forma (1.2.3). Note ainda que se $\mathcal{B}$ tem base compacta, então $\mathcal{T}_{\mathcal{F}} \subseteq \mathcal{T}_{\mathcal{B}} \subseteq \mathcal{T}_{\mathfrak{K}}$.

Em [31], McCoy e Ntantu apresentam uma abordagem por meio da noção de networks análoga a que usamos com as bornologias para definir topologias em espaços de funções . Uma família $\mathcal{N}$ de subconjuntos não vazios de $X$ é uma network se para todo $x \in X$ e $U \subseteq X$ aberto que contém $x$ existe $A \in \mathcal{N}$ tal que $x \in A \subseteq U$. Uma network $\mathcal{N}$ é fechada (compacta, etc.) se todo elemento de $\mathcal{N}$ for fechado (compacto, etc.) - note que uma network aberta é uma base para a topologia de $X$.

Como uma bornologia $\mathcal{B}$ é uma cobertura para $X$ fechada por inclusões, é imediato que toda bornologia é uma network. Analogamente, se $\mathcal{N}$ é uma network compacta (fechada) em 
$X$, então $\mathcal{N}$ é sub-base compacta (respectivamente, fechada) para a bornologia $\mathcal{B}(\mathcal{N})$ em $X-$ em particular, se $\mathcal{N}$ é fechada por inclusão e por reuniões finitas, então $\mathcal{N}$ é uma bornologia.

Sejam $Y$ um espaço topológico e $\mathcal{N}$ uma network em $X$. Em [31], os autores consideram conjuntos da forma

$$
[N, V]:=\{f \in C(X, Y): f[N] \subseteq V\}
$$

para $N \in \mathcal{N}$ e $V \subseteq Y$ aberto (compare com os conjuntos definidos em (1.2.2) e (1.3.2)). Para uma network fechada ${ }^{2} \mathcal{N}$, os autores definem $\tau_{\mathcal{N}}$, a topologia sobre $C(X, Y)$ gerada pela sub-base cujos elementos são conjuntos da forma $[N, V]$, para quaisquer $N \in \mathcal{N}$ e $V \subseteq Y$ aberto; denotaremos por $C_{\mathcal{N}}(X, Y)$ o espaço $C(X, Y)$ munido da topologia $\tau_{\mathcal{N}}$. Em particular, $\tau_{\mathcal{N}}=\tau_{\mathcal{B}(\mathcal{N})}$ para qualquer network $\mathcal{N}$.

Suponhamos agora que $X$ seja um espaço de Hausdorff munido de uma network compacta $\mathcal{N}$ e que $Y$ seja um espaço munido de uma uniformidade $\mathfrak{U}$. Nestas condições, temos

$$
C_{\mathcal{B}(\mathcal{N})}(X, Y)=C_{\mathcal{N}}(X, Y)
$$

isto é, a topologia da convergência uniforme na bornologia $\mathcal{B}(\mathcal{N})$ coincide com a topologia $\tau_{\mathcal{N}}$ em $C(X, Y)$.

Demonstração. Uma vez que os abertos básicos de $\tau_{\mathcal{N}}$ são interseções finitas de conjuntos da forma $[N, V]$, para mostrar $\tau_{\mathcal{N}} \subseteq \mathcal{T}_{\mathcal{B}(\mathcal{N})}$ em $C(X, Y)$ será suficiente provar que $[N, V] \in \mathcal{T}_{\mathcal{B}(\mathcal{N})}$. Dada uma função contínua $f \in[N, V]$, temos $f[N]$ um compacto em $Y$ contido no aberto $V$. Logo, pelo Lema 1.3.6, existe $\mathcal{U} \in \mathfrak{U}$ tal que $\mathcal{U}[f[N]] \subseteq V$. Assim, se $g \in\langle N, \mathcal{U}\rangle[f] \cap C(X, Y)$, temos $(f(x), g(x)) \in \mathcal{U}$ para todo $x \in N$, i.e., $g(x) \in \mathcal{U}[f(x)]$ para todo $x \in N$, o que acarreta $g[N] \subseteq \mathcal{U}[f[N]] \subseteq V$ e mostra que $\langle N, \mathcal{U}\rangle[f] \cap C(X, Y) \subseteq[N, V]$. Portanto, $\tau_{\mathcal{N}} \subseteq \mathcal{T}_{\mathcal{B}(\mathcal{N})}$.

Para a inclusão inversa, usaremos a igualdade $\tau_{\mathcal{N}}=\tau_{\mathcal{B}(\mathcal{N})}$.

Sejam $A \subseteq C(X, Y)$ um aberto em $C_{\mathcal{B}(\mathcal{N})}(X, Y)$ e $f \in A$. Por definição existem $B \in$ $\mathcal{B}(\mathcal{N})$ e $\mathcal{U} \in \mathfrak{U}$ tais que $\langle B, \mathcal{U}\rangle[f] \cap C(X, Y) \subseteq A$. Assim, a fim de mostrarmos que $A$

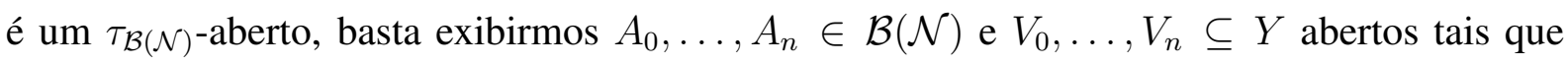
$f \in \bigcap_{i \leq n}\left[A_{i}, V_{i}\right] \subseteq\langle B, \mathcal{U}\rangle[f]$.

\footnotetext{
${ }^{2}$ Embora eles exijam que a network seja fechada, tal hipótese não é necessária, veja por exemplo a demonstração do Teorema 2.2 .6
} 
Como $\mathcal{N}$ é sub-base compacta para $\mathcal{B}(\mathcal{N})$ e $X$ é de Hausdorff, podemos supor que $B$ é compacto. Por $f$ ser contínua, temos $f[B]$ compacto em $Y$, e isso nos permite obter $x_{0}, \ldots, x_{n} \in B$ tais que $\mathcal{C}=\left\{\mathcal{V}\left[f\left(x_{i}\right)\right]: i \leq n\right\}$ recobre $f[B]$, para alguma entourage $\mathcal{V} \in \mathfrak{U}$ que determinaremos adiante, a qual podemos supor ser aberta e simétrica, como no Lema 1.3.7. Defina $A_{i}=B \cap f^{-1}\left[\mathcal{V}\left[f\left(x_{i}\right)\right]\right] \in \mathcal{B}(\mathcal{N})$ e $V_{i}=\operatorname{int}\left((\mathcal{V} \circ \mathcal{V})\left[f\left(x_{i}\right)\right]\right) \subseteq Y$, que é aberto. Claramente temos $f \in \bigcap_{i \leq n}\left[A_{i}, V_{i}\right]$ : basta notar que, como $\mathcal{V}$ é aberto em $Y \times Y$, temos $\mathcal{V}\left[f\left(x_{i}\right)\right] \subseteq \operatorname{int}\left((\mathcal{V} \circ \mathcal{V})\left[f\left(x_{i}\right)\right]\right)$. Por sua vez, se $g \in \bigcap_{i \leq n}\left[A_{i}, V_{i}\right]$, dado $x \in B$ qualquer, existe $i \leq n$ tal que $x \in A_{i}$, pois $f[B] \subseteq \mathcal{C}$ - em particular, note que $f(x) \in \mathcal{V}\left[f\left(x_{i}\right)\right]$. Daí, como $g(x) \in(\mathcal{V} \circ \mathcal{V})\left[f\left(x_{i}\right)\right]$ e $\mathcal{V}$ é simétrico, inferimos que $(f(x), g(x)) \in \mathcal{V} \circ \mathcal{V} \circ \mathcal{V}$. Portanto, basta tomar $\mathcal{V} \in \mathfrak{U}$ aberto e simétrico satisfazendo $\mathcal{V} \circ \mathcal{V} \circ \mathcal{V} \subseteq \mathcal{U}$, o que novamente podemos fazer pelo Lema 1.3.7.

Em particular, segue de 2.1.11) que se $X$ é um espaço de Hausdorff e $Y$ é um espaço uniforme, então $C_{k}(X, Y)=C_{\mathfrak{K}}(X, Y)$. Isso prova, entre outras coisas ${ }^{3}$, que as topologias da forma $\mathcal{T}_{\mathcal{B}}$ generalizam as topologias compacto-aberta e da convergência pontual, como já havíamos adiantado.

A partir daqui, deixamos de considerar todo o espaço $\left(Y^{X}, \mathcal{T}_{\mathcal{B}}\right)$ e nos focamos no subespaço $C_{\mathcal{B}}(X, Y)$ - exceto, possivelmente, para alguns fatos importantes concernentes ao espaço $Y^{X}$ munido da topologia produto. Assim, para não carregar desnecessariamente o texto, vamos utilizar a mesma notação $\langle B, \mathcal{U}\rangle$ para as entourages básicas, embora estejamos tratando apenas das funções contínuas. Mais precisamente, de agora em diante declaramos

$$
\langle B, \mathcal{U}\rangle:=\{(f, g) \in C(X, Y) \times C(X, Y): \forall x \in B((f(x), g(x)) \in \mathcal{U})\}
$$

Além disso, quando a uniformidade $\mathfrak{U}$ sobre $Y$ for metrizável, denotaremos os conjuntos da forma $\left\langle B, \mathcal{V}_{\varepsilon}\right\rangle$ simplesmente por $\langle B, \varepsilon\rangle$. Note que, em tal caso, não há perda de generalidade em supor que $\mathcal{B}$ tem base fechada, pois $\mathcal{T}_{\mathcal{B}}=\mathcal{T}_{\overline{\mathcal{B}}}$, visto que $\left\langle B, \frac{\varepsilon}{3}\right\rangle \subseteq\langle\bar{B}, \varepsilon\rangle \subseteq\langle B, \varepsilon\rangle$ pela continuidade das funções consideradas.

\footnotetext{
${ }^{3}$ Nas seções seguintes, muitos dos teoremas que veremos são adaptações de resultados de McCoy e Ntantu [31], provados originalmente para networks. Contudo, em tais resultados os autores fazem a suposição de que as networks são compactas e fechadas tanto por inclusão quanto por reuniões finitas, e assim são em verdade bornologias com base compacta.
} 
Encerramos esta subseção ressaltando alguns fatos básicos sobre $C_{\mathcal{B}}(X)$.

(1) $C_{\mathcal{B}}(X)$ é homogêneo.

Uma importante propriedade que usaremos de $C_{\mathcal{B}}(X)$ é sua homogeneidade. Um espaço topológico $X$ é um espaço homogêneo se para quaisquer $x, y \in X$ distintos existir um homeomorfismo $\varphi_{x, y}: X \rightarrow X$ tal que $\varphi_{x, y}(x)=y$ (equivalentemente, fixado $x_{0} \in X, X$ é homogêneo se, e somente se, para todo $x \in X$ existir um homeomorfismo $\varphi_{x}: X \rightarrow X$ tal que $\left.\varphi_{x}\left(x_{0}\right)=x\right)$.

Se $X$ é um espaço homogêneo e $\mathcal{P}$ é uma propriedade topológica local, então um ponto de $X$ tem $\mathcal{P}$ se, e somente se, todos os pontos de $X$ têm $\mathcal{P}$ : essencialmente, podemos "carregar" a propriedade $\mathcal{P}$ por meio dos homeomorfismos existentes devido à homogeneidade.

Para ver que $C_{\mathcal{B}}(X)$ é um espaço homogêneo, basta notar que a função

$$
\varphi_{f}: C_{\mathcal{B}}(X) \rightarrow C_{\mathcal{B}}(X)
$$

dada por $\varphi_{f}(g)=f+g$, é contínua. Daí, como tal função tem como inversa a função $\varphi_{(-f)}$ definida de maneira análoga, segue que $\varphi_{f}$ é um homeomorfismo que satisfaz $\varphi_{f}(\underline{0})=f$, onde $\underline{0}: X \rightarrow \mathbb{R}$ é a função nula.

(2) Distributividade do produto de espaços da forma $C_{\mathcal{B}}(X)$.

Em $C_{p}$-teoria, um fato conhecido sobre espaços da forma $C_{p}(X)$ trata da "distributividade" de tais espaços com relação ao produto. Adaptamos a demonstração dada em [47] por Tkachuk, e provamos a seguir uma versão válida para “ $C_{\mathcal{B}}$-teoria” em geral.

Proposição 2.1.7. Sejam $\left\{\left(X_{i}, \tau_{i}\right): i \in I\right\}$ uma família de espaços topológicos dois a dois disjuntos e $\left\{\mathcal{B}_{i}: i \in I\right\}$ tal que $\mathcal{B}_{i}$ é uma bornologia sobre $X_{i}$, para cada $i \in I$. Então $\prod_{i \in I} C_{\mathcal{B}_{i}}\left(X_{i}\right)$ é homeomorfo a $C_{\mathcal{B}}\left(\sum_{i \in I} X_{i}\right)$, onde $\mathcal{B}=\bigoplus_{i \in I} \mathcal{B}_{i}$.

Demonstração. Vamos chamar $X=\sum_{i \in I} X_{i}$. Note que $f \uparrow X_{i} \in C_{\mathcal{B}_{i}}\left(X_{i}\right)$ para quaisquer $i \in I$ e $f \in C_{\mathcal{B}}(X)$. Agora, para cada $f \in C_{\mathcal{B}}(X)$ definimos $\varphi(f)=\left(f \uparrow X_{i}\right)_{i \in I}$ e, para toda função $g \in \prod_{i \in I} C_{\mathcal{B}_{i}}\left(X_{i}\right)$ fazemos $\psi(g): X \rightarrow \mathbb{R}$ pondo $\psi(g)(x)=g(i)(x)$, onde $i \in I$ é o 
único tal que $x \in X_{i}$; como cada $g(i)$ é contínua segue que $\psi(g) \in C_{\mathcal{B}}(X)$, e assim obtemos as funções

$$
\varphi: C_{\mathcal{B}}(X) \rightarrow \prod_{i \in I} C_{\mathcal{B}_{i}}\left(X_{i}\right) \text { e } \psi: \prod_{i \in I} C_{\mathcal{B}_{i}}\left(X_{i}\right) \rightarrow C_{\mathcal{B}}(X)
$$

inversas uma da outra. Vamos provar que tanto $\varphi$ quanto $\psi$ são contínuas.

Para $f \in C_{\mathcal{B}}(X)$, seja $V=\prod_{i \in I} V_{i}$ um aberto básico de $\varphi(f)$ e chame $\operatorname{supp}(V)=$ $\left\{V_{i_{0}}, \ldots, V_{i_{n}}\right\}$. Como $f \uparrow X_{i_{j}} \in V_{i_{j}}$ para cada $j \leq n$, podemos supor $V_{i_{j}}=\left\langle B_{i_{j}}, \varepsilon_{i_{j}}\right\rangle\left[f \uparrow X_{i_{j}}\right]$, para certos $B_{i_{j}} \in \mathcal{B}_{i_{j}}$ e $\varepsilon_{i_{j}}>0$. Chamando $B_{i}=\emptyset$ se $i \notin\left\{i_{0}, \ldots, i_{n}\right\}$, é fácil ver que se $h \in\left\langle\bigsqcup_{i \in I} B_{i}, \min \left\{\varepsilon_{i_{j}}: j \leq n\right\}\right\rangle[f]$, então $\varphi(h) \in V$. Portanto, $\varphi$ é contínua.

Analogamente, tomando $g \in \prod_{i \in I} C_{\mathcal{B}_{i}}\left(X_{i}\right)$ e uma vizinhança $\left\langle\bigsqcup_{i \in I} B_{i}, \varepsilon\right\rangle[\psi(g)]$ de $\psi(g)$ , basta definir $V_{i}=C_{\mathcal{B}_{i}}\left(X_{i}\right)$ se $B_{i}=\emptyset$ e $V_{i}=\left\langle B_{i}, \varepsilon\right\rangle[g(i)]$ caso contrário, donde segue que $V=\prod_{i \in I} V_{i}$ é uma vizinhança de $g$ que atesta a continuidade de $\psi$ em $g$.

Da proposição acima segue que a menos de homeomorfismo, $\prod_{i \in I} C_{p}\left(X_{i}\right)=C_{p}\left(\sum_{i \in I} X_{i}\right)$ e, se cada $X_{i}$ for de Hausdorff, então $\prod_{i \in I} C_{k}\left(X_{i}\right)=C_{k}\left(\sum_{i \in I} X_{i}\right)$.

(3) Os espaços de Tychonoff "capturam” toda a $C_{\mathcal{B}}$-teoria.

Mostraremos que não há perda de generalidade em supor que $X$ é um espaço de Tychonoff ao tratarmos dos aspectos topológicos de $C_{\mathcal{B}}(X)$. No Problema 100 de [47], Tkachuk mostra que se $X$ é um espaço topológico, então existe um espaço de Tychonoff $Y$ tal que $C_{p}(X)$ e $C_{p}(Y)$ são topologicamente isomorfos, no seguinte sentido: existe um homeomorfismo $\varphi$ : $C_{p}(X) \rightarrow C_{p}(Y)$ que preserva a soma e a multiplicação de funções. Em nosso caso, tanto o espaço $Y$ quanto a bijeção $\varphi$ serão os mesmos apresentados em [47] e, por isso, não nos aprofundaremos em tais detalhes.

Primeiramente, consideramos uma relação de equivalência em $X$ definida por

$$
x \equiv y \Leftrightarrow \forall f \in C(X)(f(x)=f(y)) .
$$

Seja $X_{c}$ o conjunto das classes de equivalência de $\equiv$ e, para cada $f \in C(X)$, defina $\varphi_{f}$ : $X_{c} \rightarrow \mathbb{R}$ pondo $\varphi_{f}(y)=f(x)$, onde $x$ é qualquer elemento de $y$; note que $\varphi_{f}$ está bem definida. Chamando de $\tau_{R}$ a topologia usual de $\mathbb{R}$, a família $\tau_{f}=\left\{\varphi^{-1}[V]: V \in \tau_{R}\right\}$ é uma topologia sobre $X_{c}$ e $\bigcup_{f \in C(X)} \tau_{f}$ gera como sub-base uma topologia de Tychonoff $\rho$ sobre $X_{c}$; fazendo 
$Y=\left(X_{c}, \rho\right)$, segue que $\varphi_{f} \in C(Y)$ para cada $f \in C(X)$, bem como é contínua a projeção $\pi: X \rightarrow Y$ dada por $\pi(x)=[x]$. A função $\varphi: C(X) \rightarrow C(Y)$ definida por $\varphi(f)=\varphi_{f}$ é uma bijeção que satisfaz $\varphi(f+g)=\varphi(f)+\varphi(g)$ e $\varphi(f \cdot g)=\varphi(f) \cdot \varphi(g)$; em particular, $\varphi^{-1}(\xi)=\xi \circ \pi$ para cada $\xi \in C(Y)$.

Neste ponto, nossa argumentação diverge da apresentada por Tkachuk pois precisamos definir uma bornologia sobre $Y$ que esteja relacionada $\operatorname{com} \mathcal{B}$ de maneira a garantir que $\varphi$ seja um homeomorfismo. Os conjuntos da forma $\pi[B]$, para cada $B \in \mathcal{B}$, geram como sub-base uma bornologia sobre $X_{c}$, pois $X_{c}=\bigcup_{B \in \mathcal{B}} \pi[B]$; vamos denotar tal bornologia por $\pi(\mathcal{B})$; note que se $\mathcal{B}$ tem base compacta, então $\pi(\mathcal{B})$ tem base compacta, pois $\pi$ é contínua. Assim, queremos provar que $\varphi:\left(C(X), \mathcal{T}_{\mathcal{B}}\right) \rightarrow\left(C(Y), \mathcal{T}_{\pi(\mathcal{B})}\right)$ é um homeomorfismo.

Para mostrar a continuidade de $\varphi$ é suficiente notar que $\varphi^{-1}[U]$ é aberto para todo $U$ da forma $\langle\pi[B], \varepsilon\rangle[\varphi(f)]$, com $f \in C_{\mathcal{B}}(X)$. Neste caso, se $g \in\langle B, \varepsilon\rangle[f]$, então para $y \in \pi[B]$ existe $x \in B$ tal que $y=[x] \mathrm{e}$, assim, $|\varphi(f)(y)-\varphi(g)(y)|=|f(x)-g(x)|<\varepsilon$, donde temos a continuidade de $\varphi$ em $f$. Analogamente, para $\xi \in C(Y)$ e uma vizinhança $\langle B, \varepsilon\rangle[\xi \circ \pi]$ de $\varphi^{-1}(\xi)$, se $\eta \in\langle\pi[B], \varepsilon\rangle[\xi]$, então $|\xi([x])-\eta([x])|<\varepsilon$ para todo $x \in B$, donde segue que $\varphi^{-1}(\eta) \in\langle B, \varepsilon\rangle[\xi \circ \pi]$. Logo, $\varphi^{-1}$ é contínua em $\xi$.

\subsection{Propriedades de enumerabilidade em $C_{\mathcal{B}}(X)$}

Nesta seção, caracterizamos algumas funções cardinais em $C_{\mathcal{B}}(X)$ relacionadas aos axiomas de enumerabilidade. Em particular, veremos que certas propriedades de enumerabilidade em $C_{\mathcal{B}}(X)$ se "traduzem" como propriedades de metrizabilidade, tanto em $X$ quanto em $C_{\mathcal{B}}(X)$.

No primeiro capítulo, apresentamos os axiomas de enumerabilidade para um espaço topológico, e adiantamos que eles seriam generalizados por funções cardinais. É isso o que fazemos a seguir.

Sejam $X$ um espaço topológico e $x \in X$.

(a) O caráter do ponto $x$ em $X$ é o menor cardinal transfinito da forma $|\mathfrak{B}(x)|$, onde $\mathfrak{B}(x)$ é uma base local para $X$ no ponto $x$, o qual denotamos por $\chi(x, X)$. O caráter do espaço $X$ é o cardinal $\chi(X):=\sup \{\chi(x, X): x \in X\}$. 
(b) O peso do espaço $X$, denotado por $w(X)$, é o menor cardinal transfinito da forma $|\mathfrak{B}|$, onde $\mathfrak{B}$ é uma base para a topologia de $X$.

(c) A densidade de um espaço topológico $X$, denotado por $d(X)$, é o menor cardinanl transfinito da forma $|D|$, onde $D$ é um subespaço denso em $X$.

Pelas definições acima, é fácil ver que as funções cardinais caráter, peso e densidade generalizam, respectivamente, o primeiro axioma de enumerabilidade, o segundo axioma de enumerabilidade e a condição para ser separável. Além disso, é claro que $\chi(x, X) \leq w(X)$ para todo $x \in X$, bem como $d(X) \leq w(X)$. Vamos caracterizar tais funções cardinais em $C_{\mathcal{B}}(X)$, em termos de outras funções cardinais no espaço $X$. A menos que expresso o contrário, ao longo desta seção, $(M, d), X$ e $\mathcal{B}$ denotam, respectivamente, um espaço métrico $M$ cuja métrica é $d$, um espaço de Tychonoff $f^{4} X$ e uma bornologia $\mathcal{B}$ sobre $X$.

\subsubsection{O caráter de $C_{\mathcal{B}}(X)$}

Para caracterizar o caráter de $C_{\mathcal{B}}(X)$, definimos uma função cardinal no espaço $X$ diretamente relacionada com a bornologia $\mathcal{B}$. Adaptando a definiçao dada por McCoy e Ntantu [31], chamamos de grau de $\mathcal{B}$-Arens de $X$ o cardinal $a_{\mathcal{B}}(X)$ dado por

$$
a_{\mathcal{B}}(X):=\min \{|\mathcal{C}|: \mathcal{C} \text { é uma base para } \mathcal{B}\}+\aleph_{0}
$$

Teorema 2.2.1 (McCoy e Ntantu, [31], Teorema 4.4.1). Suponha que $\mathcal{B}$ tenha base fechada. Então $\chi\left(C_{\mathcal{B}}(X, M)\right) \leq a_{\mathcal{B}}(X)$ para qualquer espaço métrico $M$. Em particular, $\chi\left(C_{\mathcal{B}}(X)\right)=$ $a_{\mathcal{B}}(X)$.

Demonstração. Suponha $a_{\mathcal{B}}(X)=\kappa$ e tome $\mathcal{B}^{\prime}=\left\{B_{\alpha}: \alpha<\kappa\right\}$ uma base para $\mathcal{B}$. Para mostrar que $\chi\left(C_{\mathcal{B}}(X, M)\right) \leq \kappa$, basta notar que para qualquer função contínua $f: X \rightarrow M$, a família $\left\{\left\langle B_{\alpha}, \frac{1}{n+1}\right\rangle[f]: \alpha<\kappa\right.$ e $\left.n \in \omega\right\}$ é um sistema fundamental de vizinhanças para $C_{\mathcal{B}}(X, M)$ em $f$.

Por outro lado, assuma $\chi\left(C_{\mathcal{B}}(X)\right)=\lambda$ e tome uma base local $\left\{V_{\alpha}\right\}_{\alpha<\lambda}$ para $C_{\mathcal{B}}(X)$ em $\underline{0}$. Para cada $\alpha<\lambda$, podemos obter um fechado $B_{\alpha} \in \mathcal{B}$ e $\varepsilon_{\alpha}>0$ tais que $\left\langle B_{\alpha}, \varepsilon_{\alpha}\right\rangle[\underline{0}] \subseteq V_{\alpha}$.

\footnotetext{
${ }^{4}$ Embora muitas das definições que daremos não exijam tal hipótese de separação.
} 
Se mostrarmos que $\left\{B_{\alpha}: \alpha<\lambda\right\}$ é uma base para $\mathcal{B}$, então teremos $a_{\mathcal{B}}(X) \leq \lambda$. Ora, caso existisse $B \in \mathcal{B}$ atestando $B \not \subset B_{\alpha}$ para todo $\alpha<\lambda$, isso valeria em particular para $B_{\alpha_{0}}$, onde $\left\langle B_{\alpha_{0}}, \varepsilon_{\alpha_{0}}\right\rangle[\underline{0}] \subseteq\langle B, 1\rangle[\underline{0}]$. Daí, por $X$ ser de Tychonoff e $B \backslash B_{\alpha_{0}} \neq \emptyset$, poderíamos obter $f \in C_{\mathcal{B}}(X)$ tal que $f \uparrow B_{\alpha_{0}} \equiv 0$ e $f \notin\langle B, 1\rangle[\underline{0}]$, contrariando a inclusão anterior.

O teorema acima nos dá um critério para a metrizabilidade de $C_{\mathcal{B}}(X, M)$ :

Corolário 2.2.2 (Adaptado de Beer e Levi, [6], Teorema 7.1). Se $\mathcal{B}$ tem base fechada, então são equivalentes:

(a) $C_{\mathcal{B}}(X, M)$ é metrizável para todo espaço métrico $M$;

(b) $C_{\mathcal{B}}(X)$ é metrizável;

(c) $\chi\left(C_{\mathcal{B}}(X)\right)=\aleph_{0}$

(d) $a_{\mathcal{B}}(X)=\aleph_{0}$

Demonstração. Em vista do teorema anterior, basta mostrarmos que se $a_{\mathcal{B}}(X)=\aleph_{0}$, então $C_{\mathcal{B}}(X, M)$ é metrizável. Se $\mathcal{B}^{\prime}=\left\{B_{n}: n \in \omega\right\}$ é uma base enumerável para $\mathcal{B}$, então $\left\{\left\langle B_{m}, \frac{1}{n+1}\right\rangle: m, n \in \omega\right\}$ é uma base enumerável para a uniformidade $\mathfrak{U}(\mathcal{B})$ que gera $\mathcal{T}_{\mathcal{B}}$. Isso acarreta a pseudo-metrizabilidade de $\mathfrak{U}(\mathcal{B})$ e, por conseguinte, $C_{\mathcal{B}}(X, M)$ é pseudo-metrizável (cf. Proposição 1.3.4). Como $M$ é de Hausdorff, segue que $\mathcal{T}_{\mathcal{B}}$ é de Hausdorff e, por conseguinte, $C_{\mathcal{B}}(X, M)$ é metrizável ( $c f$. Proposição 1.3.5).

Corolário 2.2.3. Para todo espaço $X,|X|=\chi\left(C_{p}(X)\right)$.

Demonstração. Como $C_{p}(X)=C_{\mathcal{F}}(X)$, basta mostrarmos que $a_{\mathcal{F}}(X)=|X|$, mas $\mathcal{F}=$ $[X]^{<\omega}$ é a única base possível para $\mathcal{F}$, e como $\left|[X]^{<\omega}\right|=|X|$, o resultado está demonstrado.

1. Como $C_{p}(X)=C_{\mathcal{F}}(X)$, fazendo $\mathcal{B}=\mathcal{F}$ no Corolário 2.2.2, inferimos que as seguintes afirmações são equivalentes:

(a) $C_{p}(X, M)$ é metrizável para qualquer espaço métrico $M$;

(b) $\chi\left(C_{p}(X)\right)=\aleph_{0}$;

(c) $X$ é enumerável. 
2. Por sua vez, como $C_{k}(X)=C_{\mathfrak{K}}(X)$, ao fazermos $\mathcal{B}=\mathfrak{K}$, o Corolário 2.2.2 implica que são equivalentes:

(a) $C_{k}(X, M)$ é metrizável para qualquer espaço métrico $M$;

(b) $\chi\left(C_{k}(X)\right)=\aleph_{0}$;

(c) Existe uma família $\mathcal{K}_{0}$ enumerável de compactos de $X$ tal que todo compacto de $X$ está contido em algum membro de $\mathcal{K}_{0}$ (i.e., $X$ é um espaço hemicompacto)

Se $X$ é um espaço hemicompacto, então a métrica de $C_{k}(X, M)$ pode ser dada por

$$
\delta(f, g)=\sum_{n=0}^{\infty} \min \left\{\frac{1}{2^{n+1}}, \sup _{x \in K_{n}} d(f(x), g(x))\right\}
$$

onde $\left\{K_{n}: n \in \omega\right\}$ é a família cofinal de compactos de $X$ (cf. Willard [50], Problma 43G).

Se exigirmos que $\mathcal{B}$ tenha base compacta, podemos melhorar o Corolário 2.2.2 e obter novas equivalências com classes mais específicas de espaços. Para um subespaço fechado $A$ de $X$, dizemos que uma coleção de abertos $\mathfrak{B}$ é uma base local em $A$ se para todo $V \subseteq X$ aberto com $A \subseteq V$ existe $U \in \mathfrak{B}$ tal que $A \subseteq U \subseteq V$. Dizemos que $X$ é um espaço ponto-contável (point-countable-type space) se cada ponto de $X$ está contido num subespaço compacto de $X$ que possui uma base local enumerável (cf. espaço de tipo contável, no Capítulo 4); dizemos que $X$ é um q-espaço se para cada ponto $x$ de $X$ existe uma coleção $\left\{U_{n}: n \in \omega\right\}$ de vizinhanças de $x$ tal que toda família $\left\{x_{n}: n \in \omega\right\} \operatorname{com} x_{n} \in U_{n}$ tem ponto de acumulação.

Proposição 2.2.4. Seja X um espaço topológico e considere as afirmações:

(a) X é metrizável;

(b) $\chi(X)=\aleph_{0}$;

(c) X é um espaço ponto-contável;

(d) X é um q-espaço.

Então vale $(a) \Rightarrow(b) \Rightarrow(c) \Rightarrow(d)$. 
Demonstração. Provamos apenas $(c) \Rightarrow(d)$, pois as demais implicações são de verificação imediata.

Fixe $x \in X$ e tome $K \subseteq X$ um compacto que contém $x$ para o qual existe uma base local enumerável, digamos $\mathfrak{B}=\left\{U_{n}: n \in \omega\right\}$, que vamos supor decrescente com respeito à inclusão. Afirmamos que $\mathfrak{B}$ é a família de vizinhanças procurada. De fato, se $A=\left\{x_{n}: n \in \omega\right\}$ é tal que $x_{n} \in U_{n}$ para todo $n$ e $A$ não possui ponto de acumulação em $X$, então todo ponto $y \in K$ não é ponto de acumulação de $A$, donde podemos obter uma vizinhança $O_{y}$ de $y$ tal que $\left|A \cap O_{y}\right| \leq 1$, consequentemente, a compacidade de $K$ nos daria uma vizinhança $O$ de $K$ contendo no máximo finitos elementos de $A$, o que contraria o fato de $\mathfrak{B}$ ser uma base decrescente.

Mostraremos que se $\mathcal{B}$ tem uma base compacta e enumerável, então $C_{\mathcal{B}}(X)$ pertence às duas classes definidas acima.

Teorema 2.2.5 (McCoy e Ntantu, [31], Teorema 4.4.2). Se $\mathcal{B}$ tem base compacta, então as seguintes afirmações são equivalentes:

(a) $C_{\mathcal{B}}(X, M)$ é metrizável para todo espaço métrico $M$;

(b) $C_{\mathcal{B}}(X)$ é metrizável;

(c) $\chi\left(C_{\mathcal{B}}(X)\right)=\aleph_{0}$;

(d) $C_{\mathcal{B}}(X)$ é um espaço ponto-contável;

(e) $C_{\mathcal{B}}(X)$ é um q-espaço;

(f) $a_{\mathcal{B}}(X)=\aleph_{0}$

Demonstração. Em vista dos resultados já mostrados, basta provarmos que $(\mathrm{e}) \Rightarrow(\mathrm{f})$.

Suponha que $C_{\mathcal{B}}(X)$ seja um $q$-espaço e considere $\left\{W_{n}\right\}_{n \in \omega}$ uma sequência de vizinhanças de $\underline{0}$ como na definição de $q$-espaço. Para cada $n \in \omega$, existe $B_{n} \in \mathcal{B}$ compacto e $\varepsilon_{n}>0$ tal que $\left\langle B_{n}, \varepsilon_{n}\right\rangle[\underline{0}] \subseteq W_{n}$. Afirmamos que $\mathcal{B}^{\prime}=\left\{B_{n}: n \in \omega\right\}$ é uma base para $\mathcal{B}$.

Se não for este o caso, então existe $B \in \mathcal{B}$ compacto satisfazendo $B \backslash B_{n} \neq \emptyset$ para todo $n \in \omega$, o que nos permite escolher $x_{n} \in B \backslash B_{n}$. Como $x_{n}$ é um ponto não pertencente ao compacto $B_{n}$, podemos obter uma função contínua $g_{n}: X \rightarrow \mathbb{R}$ tal que $g_{n}\left(x_{n}\right)=n+1$ e $g_{n} \uparrow$ 
$B_{n} \equiv 0$ (pois $X$ é de Tychonoff e $B_{n}$ é fechado). Por construção, cada $g_{n} \in\left\langle B_{n}, \varepsilon_{n}\right\rangle[\underline{0}] \subseteq W_{n}$, e a hipótese garante a existência de algum ponto de acumulação $f \in C_{\mathcal{B}}(X)$ para $\left(g_{n}\right)_{n \in \omega}$, o que é impossível. De fato, se existisse $f$ ponto de acumulação de $\left(g_{n}\right)_{n \in \omega}$, então existiria uma subsequência (infinita) $\left(g_{n_{k}}\right)_{k \in \omega}$ contida em $\langle B, 1\rangle[f]$, e assim

$$
1>\left|g_{n_{k}}\left(x_{n_{k}}\right)-f\left(x_{n_{k}}\right)\right| \geq n_{k}+1-\left|f\left(x_{n_{k}}\right)\right|
$$

donde $\left|f\left(x_{n_{k}}\right)\right|>n_{k}$, mostrando que $f\lceil B$ não é limitada, o que é absurdo, pois $f$ é contínua e $B$ é compacto ${ }^{5}$. Logo, $\mathcal{B}^{\prime}$ é uma base enumerável para $\mathcal{B}$, como queríamos.

\subsubsection{O peso de $C_{\mathcal{B}}(X)$}

Começamos esta subseção com algumas observações de caráter geral sobre a função cardinal peso. Primeiramente, se $Y$ e $X$ são espaços topológicos, então

$$
w\left(Y^{X}\right) \leq|X| \cdot w(Y)
$$

De fato, se $\mathfrak{B}$ é uma base para a topologia de $Y$, então a coleção $\mathfrak{B}^{\prime}$ de todos os conjuntos da forma $V=\prod_{x \in X} V_{x}$, com $|\operatorname{supp}(V)|<\aleph_{0}$ e $V_{x} \in \mathfrak{B}$ sempre que $V_{x} \neq Y$, é uma base para a topologia de $Y^{X}$, e $\left|\mathfrak{B}^{\prime}\right| \leq\left|[X]^{<\omega}\right| \cdot\left|[\mathfrak{B}]^{<\omega}\right|=|X| \cdot|\mathfrak{B}|$.

A segunda observação consiste de um "limitante superior" para o peso de um espaço uniforme. Para um espaço uniforme $(Y, \mathfrak{U})$, se considerarmos sobre $Y$ a topologia induzida por $\mathfrak{U}$, então

$$
w(Y) \leq d(Y) \cdot w(\mathfrak{U})
$$

onde $w(\mathfrak{U})$ é o menor cardinal da forma $|\mathfrak{B}|$, com $\mathfrak{B}$ sendo uma base para a uniformidade $\mathfrak{U}$.

Demonstração. Seja $\mathscr{U}=\left\{\mathcal{U}_{i}: i \in I\right\}$ uma base para $\mathfrak{U}$ e considere $D \subseteq Y$ denso. Se provarmos que $\mathfrak{B}=\left\{\mathcal{U}_{i}[d]: i \in I, d \in D\right\}$ é um sistema fundamental de vizinhanças para a topologia de $Y$, então a desigualdade estará demonstrada. Em vista do Lema 1.3.7, não há perda de generalidade em supor que os elementos de $\mathscr{U}$ sejam simétricos. Agora, para $y \in Y$ e $V \subseteq Y$ aberto com $y \in V$, existem $\mathcal{U} \in \mathfrak{U}$ e $i \in I$ tais que $y \in(\mathcal{U} \circ \mathcal{U})[y] \subseteq V$ e $\mathcal{U}_{i} \subseteq \mathcal{U}$. Como

\footnotetext{
${ }^{5}$ Apenas aqui usamos a hipótese de que $\mathcal{B}$ tem base compacta.
} 
$D$ é denso, existe $d \in D \cap \mathcal{U}_{i}[y]$, isto é, $(y, d) \in \mathcal{U}_{i} \subseteq \mathcal{U}$, o que nos dá $y \in \mathcal{U}_{i}[d]$ pela simetria de $\mathcal{U}_{i}$. Para $w \in \mathcal{U}_{i}[d]$ qualquer, temos $(y, d),(d, w) \in \mathcal{U}_{i} \subseteq \mathcal{U}$, donde segue que $(y, w) \in \mathcal{U} \circ \mathcal{U}$ e, assim, $w \in(\mathcal{U} \circ \mathcal{U})[y] \subseteq V$. Logo, $y \in \mathcal{U}_{i}[d] \subseteq V$, mostrando que $\mathfrak{B}$ é um sistema fundamental de vizinhanças para a topologia de $Y$, como queríamos.

Para o contexto da $C_{\mathcal{B}}$-teoria, a observação acima nos dá

$$
w\left(C_{\mathcal{B}}(X)\right) \leq d\left(C_{\mathcal{B}}(X)\right) \cdot a_{\mathcal{B}}(X) \leq w\left(C_{\mathcal{B}}(X)\right)
$$

pois, se $\mathcal{B}^{\prime} \subseteq \mathcal{B}$ é base para a bornologia $\mathcal{B}$, então $\left\{\left\langle B, \frac{1}{n+1}\right\rangle: B \in \mathcal{B}^{\prime}, n \in \omega\right\}$ é base para a uniformidade $\mathfrak{U}(\mathcal{B})$ que gera $\mathcal{T}_{\mathcal{B}}$, acarretando $w(\mathfrak{U}(\mathcal{B})) \leq a_{\mathcal{B}}(X)=\chi\left(C_{\mathcal{B}}(X)\right) \leq w\left(C_{\mathcal{B}}(X)\right)$.

Agora, o net-peso do espaço $X$, denotado por $n w(X)$, é o menor cardinal transfinito da forma $|\mathcal{N}|$, onde $\mathcal{N}$ é uma network em $X$. Claramente, tem-se $d(Y) \leq n w(Y) \leq w(Y)$ para qualquer espaço $Y$. Logo, em vista de 2.2.4), temos $w(Y)=d(Y)=n w(Y)$ para qualquer espaço $Y$ pseudo-metrizável ( $c f$. Proposição 1.3.4). Em particular, temos

$$
w\left(C_{\mathcal{B}}(X)\right)=d\left(C_{\mathcal{B}}(X)\right) \cdot a_{\mathcal{B}}(X)=n w\left(C_{\mathcal{B}}(X)\right) \cdot a_{\mathcal{B}}(X)
$$

Assim, para caracterizarmos $w\left(C_{\mathcal{B}}(X)\right)$ em termos de propriedades de $X$, devemos avaliar mais detalhadamente o net-peso ou a densidade de $C_{\mathcal{B}}(X)$. Como a próxima subseção tratará da densidade de $C_{\mathcal{B}}(X)$, vamos agora estudar com mais cuidado as networks de $C_{\mathcal{B}}(X)$.

Teorema 2.2.6 (McCoy e Ntantu, [31], Teorema 4.1.1). Se $\mathcal{B}$ tem base compacta, então

$$
n w\left(C_{\mathcal{B}}(X)\right) \leq w(X)
$$

Demonstração. Primeiramente, note que se $\tau$ e $\tau^{\prime}$ são topologias sobre um conjunto $Y$ e $\tau \subseteq \tau^{\prime}$, então $n w(Y, \tau) \leq n w\left(Y, \tau^{\prime}\right)$ : basta ver que se $\mathcal{N}$ é uma network para $\left(Y, \tau^{\prime}\right)$, então $\mathcal{N}$ é uma network para $(Y, \tau)$. Vamos obter uma topologia $\tau$ sobre $C(X)$ tal que $\mathcal{T}_{\mathcal{B}} \subseteq \tau$ em $C(X)$ e $n w(C(X), \tau) \leq w(X)$. Assim, a desigualdade $n w\left(C_{\mathcal{B}}(X)\right) \leq w(X)$ seguirá de imediato.

Sejam $\mathcal{V}$ uma base para a topologia de $X \operatorname{com}|\mathcal{V}|=w(X)$ e $\mathcal{W}$ uma base enumerável para a topologia de $\mathbb{R}$. Chamaremos de $\tau$ a topologia sobre $C(X)$ que tem como sub-base os 
conjuntos da forma $[V, W]:=\{f \in C(X): f[V] \subseteq W\}$ para $V \in \mathcal{V}$ e $W \in \mathcal{W}$. Assim, já temos

$$
n w(C(X), \tau) \leq w(C(X), \tau) \leq w(X) \cdot \aleph_{0}=w(X)
$$

Resta mostrar que $\mathcal{T}_{\mathcal{B}} \subseteq \tau$ em $C(X)$.

Sejam $A \subseteq C_{\mathcal{B}}(X)$ aberto, $f \in A$ e tome $B \in \mathcal{B}$ e $\varepsilon>0$ tais que $\langle B, \varepsilon\rangle[f] \subseteq A$. Como $\mathcal{B}$ tem base compacta, não há perda de generalidade em supor $B$ compacto. Para cada $b \in B$, podemos obter $V_{b} \in \mathcal{V}$ e $W_{b} \in \mathcal{W}$ tais que $b \in V_{b}, f(b) \in W_{b} \subseteq B(f(b), \delta)$ e $f\left[V_{b}\right] \subseteq W_{b}$, para algum $\delta>0$ que determinaremos adiante. Da compacidade de $B$, existem $b_{0}, \ldots, b_{n} \in B$ tais que $B \subseteq \bigcup_{i \leq n} V_{b_{i}}$. Fazendo $\delta=2^{-1} \varepsilon$, temos

$$
f \in \bigcap_{i \leq n}\left[V_{b_{i}}, W_{b_{i}}\right] \subseteq\langle B, \varepsilon\rangle[f] \subseteq A
$$

Corolário 2.2.7. Se $\mathcal{B}$ tem base compacta, então $l\left(C_{\mathcal{B}}(X)\right) \leq w(X)$.

Demonstração. Basta observar que para qualquer espaço topológico $Y$ vale $l(Y) \leq n w(Y)$, donde o resultado segue pelo teorema anterior. Sejam $\mathcal{U}$ uma cobertura de abertos para $Y$ e $\mathcal{N}$ uma network em $Y$. Fixe $U \in \mathcal{U}$ e, para cada $N \in \mathcal{N}$, faça $U_{N}=U$ caso não exista $V \in \mathcal{U}$ tal que $N \subseteq V$ e, caso contrário, escolha algum $U_{N} \in \mathcal{U}$ tal que $N \subseteq U_{N}$. É fácil ver que $\mathcal{U}^{\prime}=\left\{U_{N}: N \in \mathcal{N}\right\} \subseteq \mathcal{U}$ é uma cobertura aberta para $Y$.

As networks em $C_{\mathcal{B}}(X)$ estão diretamente relacionadas com uma classe de estruturas em $X$ definidas em termos de $\mathcal{B}$ e semelhantes às networks. Adaptando a definição dada por McCoy e Ntantu em [31], dizemos que $A \subseteq \wp(X)$ é uma $\mathcal{B}$-network em $X$ se, para quaisquer $B \in \mathcal{B}$ e $V \subseteq X$ aberto com $\bar{B} \subseteq V$ existir $A \in \mathcal{A}$ tal que $\bar{B} \subseteq A \subseteq V$. O $\mathcal{B}$-net-peso do espaço $X$, denotado por $n w_{\mathcal{B}}(X)$, é o menor cardinal transfinito da forma $|\mathcal{A}|$, onde $\mathcal{A}$ é uma $\mathcal{B}$-network de $X$. Observe que, assim como $l(X) \leq n w(X)$, temos também $l_{\mathcal{B}}(X) \leq n w_{\mathcal{B}}(X)$ para qualquer bornologia $\mathcal{B}$ com base fechada.

Teorema 2.2.8 (McCoy e Ntantu, [31], Teorema 4.1.2). Se $\mathcal{B}$ tem base compacta, então

$$
n w\left(C_{\mathcal{B}}(X)\right)=n w_{\mathcal{B}}(X)
$$


Demonstração. Sejam $\mathcal{N}$ uma $\mathcal{B}$-network em $X$ e $\mathcal{W}$ a base para a topologia de $\mathbb{R}$ formada pelos intervalos abertos de centro e raio racionais. Considere a família $\mathcal{N}_{\mathcal{B}}$ cujos membros são interseções finitas dos conjuntos $[N, W]:=\left\{f \in C_{\mathcal{B}}(X): f[N] \subseteq W\right\}$, para $N \in \mathcal{N}$ e $W \in \mathcal{W}$; se mostrarmos que $\mathcal{N}_{\mathcal{B}}$ é uma network em $C_{\mathcal{B}}(X)$, então seguirá que $n w\left(C_{\mathcal{B}}(X)\right) \leq$ $n w_{\mathcal{B}}(X)$, pois $\left|\mathcal{N}_{\mathcal{B}}\right| \leq|\mathcal{N}|$. Fixemos $B \in \mathcal{B}$ compacto, $\varepsilon>0$ e $f \in C_{\mathcal{B}}(X)$. Para cada $b \in B$, pela continuidade de $f$ podemos obter $U_{b} \subseteq X$ aberto e intervalos $W_{b}, V_{b} \in \mathcal{W}$ tais que:

- $b \in U_{b}$;

- $f(b) \in W_{b}$;

- $f\left[U_{b}\right] \subseteq W_{b} \subseteq \overline{W_{b}} \subseteq V_{b} \subseteq B\left(f(b), \frac{\varepsilon}{2}\right)$.

Note que para cada $b \in B$ temos $\overline{B \cap U_{b}} \subseteq B$ e $\overline{B \cap U_{b}} \subseteq f^{-1}\left[V_{b}\right]$ e, assim, por $\mathcal{N}$ ser uma $\mathcal{B}$-network, existe $N_{b} \in \mathcal{N}$ tal que $\overline{B \cap U_{b}} \subseteq N_{b} \subseteq f^{-1}\left[V_{b}\right]$. Enfim, da compacidade de $B$ obtemos $b_{0}, \ldots, b_{n} \in B$ tais que os abertos $U_{b_{0}}, \ldots, U_{b_{n}}$ recobrem $B$. Temos então

$$
f \in \bigcap_{i \leq n}\left[N_{b_{i}}, V_{b_{i}}\right] \subseteq\langle B, \varepsilon\rangle[f]
$$

Seja agora $\mathcal{F}$ uma network em $C_{\mathcal{B}}(X)$. Afirmamos que o conjunto $\left\{F^{*}: F \in \mathcal{F}\right\}$ é uma $\mathcal{B}$-network em $X$, onde $F^{*}=\{x \in X: \forall f \in F(f(x)>0)\}$ para cada $F \in \mathcal{F}$. De fato, sejam $B \in \mathcal{B}$ e $V \subseteq X$ aberto tais que $\bar{B} \subseteq V$. Pelo Lema de Urysohn "Fraco", existe $f \in C_{\mathcal{B}}(X)$ não negativa tal que $f \uparrow \bar{B} \equiv 1$ e $f \nmid X \backslash V \equiv 0$. Como $f[\bar{B}]$ é compacto e $f[\bar{B}] \subseteq(0,+\infty)$, existe $\delta>0$ tal que $f[\bar{B}]^{\delta} \subseteq(0,+\infty)$ e, pela hipótese, existe $F \in \mathcal{F}$ satisfazendo $f \in F \subseteq\langle\bar{B}, \delta\rangle[f]$. Pela forma como tomamos $\delta$, segue que $\bar{B} \subseteq F^{*}$ e, pela construção de $f$, devemos ter $F^{*} \subseteq V$, pois $f \in F$. Portanto, $n w_{\mathcal{B}}(X) \leq n w\left(C_{\mathcal{B}}(X)\right)$.

Da caracterização do net-peso de $C_{\mathcal{B}}(X)$ e da observação 2.2.5), o seguinte corolário é imediato:

Corolário 2.2.9 (McCoy e Ntantu, [31], Teorema 4.5.2). Se B tem base compacta, então

$$
w\left(C_{\mathcal{B}}(X)\right)=a_{\mathcal{B}}(X) \cdot n w_{\mathcal{B}}(X)
$$


Corolário 2.2.10. Para todo espaço X, temos:

(a) (Arhangel'skii, apud Okuyama e Terada, [35]) $n w\left(C_{p}(X)\right)=n w(X)$;

(b) $w\left(C_{p}(X)\right)=|X|$ (em particular, $C_{p}(X)$ satisfaz o segundo axioma de enumerabilidade se, e somente se, $X$ é enumerável).

Demonstração. Em vista do último teorema, a igualdade do item $(a)$ é equivalente a $n w(X)=$ $n w_{\mathcal{F}}(X)$. Como toda $\mathcal{F}$-network é network, temos $n w(X) \leq n w_{\mathcal{F}}(X)$. Agora, se $\mathcal{N}$ é uma network (infinita), então a família $\mathcal{N}^{\prime}$ das reuniões finitas de membros de $\mathcal{N}$ é claramente uma $\mathcal{F}$-network e, como $\left|\mathcal{N}^{\prime}\right| \leq\left|[\mathcal{N}]^{<\omega}\right|=|\mathcal{N}|$, obtemos $n w_{\mathcal{F}}(X) \leq n w(X)$. Para o item $(b)$, já vimos que $|X|=\chi\left(C_{p}(X)\right)$ e $n w\left(C_{p}(X)\right)=n w(X)$. Como $n w(X) \leq|X|$, temos

$$
|X|=\chi\left(C_{p}(X)\right) \leq w\left(C_{p}(X)\right)=\chi\left(C_{p}(X)\right) \cdot n w\left(C_{p}(X)\right) \leq|X| \cdot|X|=|X| .
$$

No contexto da $C_{k}$-teoria, note que $n w_{\mathfrak{K}}(X) \leq \kappa$ se, e somente se, existe uma família $\mathcal{N}=\left\{N_{\alpha}\right\}_{\alpha<\kappa}$ de subconjuntos de $X$ tal que para quaisquer $K \subseteq X$ compacto e $V \subseteq X$ aberto com $K \subseteq V$ existe $N \in \mathcal{N}$ satisfazendo $K \subseteq N \subseteq V$, o que equivale a $n w\left(C_{k}(X)\right) \leq \kappa$ pelo Teorema 2.2.8. Segundo a terminologia de McCoy e Ntantu em [31], um espaço $X$ que satisfaz $n w_{\mathfrak{K}}(X)=\aleph_{0}$ é chamado de $\aleph_{0}$-espaço . Assim, $C_{k}(X)$ satisfaz o segundo axioma de enumerabilidade se, e somente se $X$ é um $\aleph_{0}$-espaço hemicompacto.

\subsubsection{Densidade e celularidade de $C_{\mathcal{B}}(X)$}

Nesta subseção, vamos analisar algumas propriedades topológicas de $X$ relacionadas com a densidade e com a celularidade de $C_{\mathcal{B}}(X)$, que definiremos adiante.

Para um espaço de Tychonoff $X$, definimos o peso fraco do espaço $X$ como sendo o cardinal transfinito $i w(X)$ dado por

$i w(X):=\min \{w(Y):$ existe bijeção contínua de $X$ sobre $Y$ e $Y$ é espaço de Tychonoff $\}$.

\footnotetext{
${ }^{6} \mathrm{Em}$ [31], os autores denotam $i w(X)$ por $w w(X)$, e o chamam de weak weight. Adotamos a notação $i w$ para o peso fraco por esta ser mais recorrente na literatura atual.
} 
Seja $\tau$ a topologia de $X$. Note que a asserção $i w(X)=\kappa$ é equivalente à existência de uma topologia $\tau^{\prime}$ sobre $X$ com as seguintes propriedades:

- $\left(X, \tau^{\prime}\right)$ é de Tychonof e $w\left(\left(X, \tau^{\prime}\right)\right)=\kappa$,

- $\tau^{\prime} \subseteq \tau \mathrm{e}$

- se $\sigma$ é uma topologia sobre $X$ tal que $(X, \sigma)$ é de Tychonoff e $\sigma \subseteq \tau$, então $w((X, \sigma)) \geq$ $\kappa$.

Ocorre que o peso fraco de $X$ determina completamente a densidade de $C_{\mathcal{B}}(X)$, para qualquer bornologia $\mathcal{B}$ com base compacta.

Teorema 2.2.11 (McCoy e Ntantu, [31], Teorema 4.2.1). Se $\mathcal{B}$ tem base compacta, então

$$
d\left(C_{\mathcal{B}}(X)\right)=i w(X)
$$

Demonstração. Como $\mathcal{T}_{\mathcal{F}} \subseteq \mathcal{T}_{\mathcal{B}} \subseteq \mathcal{T}_{\mathfrak{K}}$, temos $d\left(C_{p}(X)\right) \leq d\left(C_{\mathcal{B}}(X)\right) \leq d\left(C_{k}(X)\right)$. Assim, se mostrarmos que $d\left(C_{k}(X)\right) \leq i w(X) \leq d\left(C_{p}(X)\right)$, então o teorema estará demonstrado.

Provemos que $d\left(C_{k}(X)\right) \leq i w(X)$. Fixe um espaço de Tychonoff $Y \operatorname{com} w(Y)=i w(X) \mathrm{e}$ uma bijeção contínua $f: X \rightarrow Y$. Exibiremos uma função contínua $\varphi: C_{k}(Y) \rightarrow C_{k}(X)$ cuja imagem seja densa em $C_{k}(X)$; se provarmos isso, teremos

$$
d\left(C_{k}(X)\right) \leq d\left(C_{k}(Y)\right) \leq n w\left(C_{k}(Y)\right) \leq w(Y)=i w(X)
$$

pois para $D \subseteq C_{k}(Y)$ denso, teríamos $\varphi[D]$ denso em $\varphi\left[C_{k}(Y)\right]$ e, por conseguinte $\varphi[D]$ seria denso em $C_{k}(X), \operatorname{com}|\varphi[D]| \leq|D|$.

Definamos $\varphi: C_{k}(Y) \rightarrow C_{k}(X)$ fazendo $\varphi(g)=g \circ f$ para toda $g \in C_{k}(Y)$. Claramente $\varphi$ é contínua pois, se $g \in C_{k}(Y)$ e $\langle K, \varepsilon\rangle[\varphi(g)]$ é uma vizinhança de $\varphi(g)$ em $C_{k}(X)$, basta notar que $f[K]$ é compacto em $Y$ e assim $\varphi[\langle f[K], \varepsilon\rangle[g]] \subseteq\langle K, \varepsilon\rangle[\varphi(g)]$. Resta apenas mostrar que a imagem de $\varphi$ é densa em $C_{k}(X)$.

Como $f: X \rightarrow Y$ é uma bijeção contínua7, segue que para qualquer $K \subseteq X$ compacto a restrição $f \uparrow K: K \rightarrow f[K]$ é uma aplicação fechada e, por conseguinte, é um

\footnotetext{
${ }^{7}$ Seria suficiente que $f$ fosse meramente uma injeção contínua.
} 
homeomorfismo. Para $h \in C_{k}(X)$ e $\langle K, \varepsilon\rangle[h]$ uma vizinhança de $h$ em $C_{k}(X)$, a função $h \circ(f \uparrow K)^{-1}: f[K] \rightarrow \mathbb{R}$ é contínua e admite uma extensão contínua $\tilde{h}: Y \rightarrow \mathbb{R}(c f$. Corolário 1.2.2). Note por fim que $\varphi(\tilde{h}) \in\langle K, \varepsilon\rangle[h]$, pois $\varphi(\tilde{h}) \uparrow K=h \uparrow K$.

Mostremos agora que $i w(X) \leq d\left(C_{p}(X)\right)$. Seja $D \subseteq C_{p}(X)$ um denso com $|D|=$ $d\left(C_{p}(X)\right)$ e considere a função $\mu: X \rightarrow \mathbb{R}^{D}$, que a cada $x \in X$ associa a função $\mu_{x}: D \rightarrow \mathbb{R}$ dada por $\mu_{x}(f)=f(x)$, para qualquer $f \in D$. A continuidade de $\mu$ segue do fato de considerarmos $\mathbb{R}^{D}$ munido da topologia produto. Além disso, $\mu$ é injetora: de fato, se $x \neq y$ são pontos de $X$, podemos considerar abertos não vazios e disjuntos $A_{x}$ e $A_{y}$ em $\mathbb{R}$, e fazer $A_{w}=\mathbb{R}$ para todo $w \in X \backslash\{x, y\}$, o que nos dá um aberto básico $A=\prod_{u \in X} A_{u}$ e não vazio de $\mathbb{R}^{X}$; como $D$ é denso em $C_{p}(X)$ e este é denso em $\mathbb{R}^{X}$, temos $D$ denso em $\mathbb{R}^{X}$ e isso implica que existe $f \in D \cap A$, acarretando $f(x) \neq f(y)$ e, por conseguinte, $\mu_{x} \neq \mu_{y}$. Dessa forma, $\mu: X \rightarrow \mu[X]$ é uma bijeção contínua, com $\mu[X] \subseteq \mathbb{R}^{D}$ um espaço de Tychonoff. Portanto, $i w(X) \leq w(\mu[X]) \leq w\left(\mathbb{R}^{D}\right) \leq|D|=d\left(C_{p}(X)\right)$.

Corolário 2.2.12. Para um espaço de Tychonoff $(X, \tau)$, são equivalentes:

(a) $C_{p}(X)$ é separável;

(b) $C_{k}(X)$ é separável;

(c) Existe uma topologia $\tau^{\prime} \subseteq \tau$ em $X$ que é metrizável e separável.

Demonstração. Segue diretamente do teorema anterior, aliado ao fato de que um espaço tem peso enumerável e é regular se, e somente se, é metrizável e separável.

Mais geralmente, podemos estimar a densidade do espaço $\mathbb{R}^{X}$ por meio do seguinte teorema:

Teorema 2.2.13 (Teorema de Hewitt-Marczewski-Pondiczery). Sejam $\left\{X_{s}\right\}_{s \in S}$ uma família de espaços topológicos e $\kappa$ um cardinal transfinito. Se $|S| \leq 2^{\kappa}$ e d $\left(X_{s}\right) \leq \kappa$ para todo $s \in S$, então $d\left(\prod_{s \in S} X_{s}\right) \leq \kappa$.

Demonstração. Primeiro mostraremos que $d\left(\prod_{s \in S} X_{s}\right) \leq d\left(D(\kappa)^{S}\right)$, onde $D(\kappa)$ é um espaço discreto de cardinalidade $\kappa$. Para cada $s \in S$ tome um subconjunto $A_{s}$ denso em $X_{s}$ tal que $\left|A_{s}\right| \leq \kappa$ e uma função $f_{s}: D(\kappa) \rightarrow A_{s}$ sobrejetora (e contínua, pois $D(\kappa)$ é discreto). Defina 
então

$$
\begin{aligned}
\varphi: D(\kappa)^{S} & \rightarrow X:=\prod_{s \in S} X_{s} \\
x & \mapsto \varphi_{x}
\end{aligned}
$$

onde $\varphi_{x}(s)=f_{s}(x(s))$ para quaisquer $s \in S$ e $x \in D(\kappa)^{S}$. Note que a sobrejetividade de cada $f_{s}$ acarreta que $\varphi\left[D(\kappa)^{S}\right]=\prod_{s \in S} A_{s}:=A$, e a densidade de $A_{s}$ em $X_{s}$ para cada $s \in S$ nos dá $\bar{A}=X$. Assim, temos $d(X) \leq d(A)$, pois todo denso de $A$ também é denso em $X$, e consequentemente $d(A) \leq d\left(D(\kappa)^{S}\right)$, pois $A$ é imagem contínua de $D(\kappa)^{S}$. Logo, $d(X) \leq d\left(D(\kappa)^{S}\right)$, como queríamos.

Desse modo, para concluirmos a demonstração, basta provarmos que $d\left(D(\kappa)^{S}\right) \leq \kappa$. Para isso, precisamos exibir $\mathcal{D} \subseteq D(\kappa)^{S}$, com $|\mathcal{D}| \leq \kappa$, tal que $\mathcal{D}$ seja denso em $D(\kappa)^{S}$. Considerando o conjunto $T=\{0,1\}^{\kappa}$ munido da topologia produto, temos $|T|=2^{\kappa}$ e $w(T) \leq \kappa$. Como $|S| \leq|T|$, existe $i: S \rightarrow T$ uma função injetora, o que nos permite "enxergar" $S$ como um subespaço de $T$. Seja então $\mathfrak{B}$ uma base para $S$ com $|\mathfrak{B}| \leq \kappa$, e denote por $\mathcal{T}$ a família de todos os subconjuntos finitos de $\mathfrak{B}$ cujos elementos sejam dois-a-dois disjuntos (isto é, $V \in \mathcal{T}$ se, e somente se, $V$ é uma família finita de abertos de $\mathfrak{B}$ dois-a-dois disjuntos); como $\mathcal{T} \subseteq[\mathfrak{B}]^{<\omega}$, temos $|\mathcal{T}| \leq \kappa$.

Chame então

$\mathcal{D}=\left\{f \in D(\kappa)^{S}: \exists V \in \mathcal{T}(f \uparrow U\right.$ é constante para todo $U \in V$ e $f \uparrow S \backslash \bigcup V$ é constante $\left.)\right\}$.

Note que $|\mathcal{D}| \leq \kappa$ : para cada $f \in \mathcal{D}$, podemos escolher $V=\left\{U_{0}, \ldots, U_{n}\right\} \in \mathcal{T}, u_{i} \in D(\kappa)$ para cada $i \leq n$ e $a \in D(\kappa)$ tais que $f \uparrow U_{i} \equiv u_{i}$ e $f \uparrow S \backslash \bigcup V \equiv a$, o que estabelece uma injeçãd $]^{8}$ de $\mathcal{D}$ para $\mathcal{B}^{<\omega} \times D(\kappa)^{<\omega}$, e $\left|\mathcal{B}^{<\omega} \times D(\kappa)^{<\omega}\right|=\kappa$.

Finalmente, mostraremos que $\mathcal{D}$ é denso em $D(\kappa)^{S}$. Seja $V=\prod_{s \in S} V_{s}$ um aberto de $D(\kappa)^{S}$ com $|\operatorname{supp}(V)|<\aleph_{0}$, digamos $\operatorname{supp}(V)=\left\{s_{0}, \ldots, s_{n}\right\}$. Como a topologia de $S$ é de Hausdorff (por ser induzida de $T=\{0,1\}^{\kappa}$ ), existem $U_{0}, \ldots, U_{n}$ abertos dois-a-dois disjuntos com $s_{i} \in U_{i}$. Para cada $i \leq n$ escolha $u_{i} \in V_{s_{i}} \subseteq D(\kappa)$ e fixe $a \in D(\kappa)$ qualquer, e então defina $x: S \rightarrow D(\kappa)$ fazendo $x(s)=u_{i}$ se $s \in U_{i}$ e $x(s)=a$ caso contrário. Claramente, $x \in V \cap \mathcal{D}$, o que mostra a densidade de $\mathcal{D}$ em $D(\kappa)^{S}$ e encerra a demonstração.

\footnotetext{
${ }^{8}$ Por exemplo, tal injeção pode ser dada por $f \mapsto\left(\left(U_{0}, \ldots, U_{n}\right),\left(u_{0}, \ldots, u_{n}, a\right)\right)$.
} 
Suponha que $D$ seja um subespaço denso de um espaço topológico $X$, e considere $\mathcal{U}$ uma família de abertos não vazios de $X$ dois-a-dois disjuntos; note que a cardinalidade de $\mathcal{U}$ não pode exceder $|D|$, pois cada elemento de $\mathcal{U}$ deve conter ao menos um membro de $D$. Definimos a celularidade do espaço $X$ como sendo o cardinal transfinito $c(X)$ dado por

$$
c(X):=\sup \{|\mathcal{U}|: \mathcal{U} \text { é uma família de abertos não vazios de } X \text { dois a dois disjuntos }\}+\aleph_{0},
$$

e dizemos que $X$ é um espaço $\operatorname{ccc}^{9}$ se $c(X)=\aleph_{0}$. Pelo que dissemos acima, é imediato que para todo espaço $X$ vale $c(X) \leq d(X)$ e, por conseguinte:

$$
c(X) \leq d(X) \leq n w(X) \leq w(X)
$$

Em particular, no caso em que $X$ é metrizável, todas as funções cardinais acima coincidem. Para provar isso, usaremos uma formulação equivalente do Axioma da Escolha, o chamado Lema de Teichmüller-Tukey. Dados um conjunto $X$ e uma propriedade $\mathcal{P}$ relativa a subconjuntos de $X$, diremos que $\mathcal{P}$ é uma propriedade de caráter finito se o conjunto vazio tiver a propriedade $\mathcal{P}$ e for verdadeiro que $A \subseteq X$ tem a propriedade $\mathcal{P}$ se, e somente se, todo subconjunto finito de $A$ tem a propriedade $\mathcal{P}$.

Teorema 2.2.14 (Lema de Teichmüller-Tukey). Se P é uma propriedade de caráter finito referente a subconjuntos de $X$, então todo subconjunto $A \subseteq X$ com a propriedade $\mathcal{P}$ está contido num subconjunto $B \subseteq X$ que tem a propriedade $\mathcal{P}$ e é maximal (no sentido da inclusão) na família de todos os subconjuntos de $X$ que têm a propriedade $\mathcal{P}$.

Lema 2.2.15. Se $X$ é metrizável, então $c(X)=w(X)=n w(X)=d(X)$.

Demonstração. Como $X$ é metrizável, já temos $d(X)=n w(X)=w(X)(c f$. observação (2.2.4)). Logo, basta mostrarmos que $c(X) \geq d(X)$.

Seja $d$ uma métrica em $X$ e $c(X) \leq \kappa$. Para cada $n \in \omega$, considere $\mathcal{P}_{n}(A)$ a seguinte propriedade em $A \subseteq X: d(x, y) \geq \frac{1}{n+1}$ para quaisquer $x, y \in A$. É fácil ver que a propriedade $\mathcal{P}_{n}$ é de caráter finito. Como $X$ é infinito, existem $x, y \in X$ distintos com $d(x, y) \geq \frac{1}{n+1}$ para algum $n$ e, por conseguinte, $d(x, y) \geq \frac{1}{m+1}$ para todo $m \geq n$. Logo, pelo Lema de TeichmüllerTukey, segue que para cada $m \geq n$ existe $A_{m} \subseteq X$ maximal (no sentido da inclusão) na família

\footnotetext{
${ }^{9}$ ccc é abreviação para countable chain condition.
} 
de todos os conjuntos com a propriedade $\mathcal{P}_{m}$. Agora, notemos que a família

$$
\mathcal{U}_{m}=\left\{B\left(x, \frac{1}{2} \cdot \frac{1}{m+1}\right): x \in A_{m}\right\}
$$

é uma família de abertos dois a dois disjuntos e, por conseguinte, $\left|A_{m}\right| \leq \kappa$. O teorema estará provado se mostrarmos que $A=\bigcup_{m \geq n} A_{n}$ é denso em $X$. Mas, se $x \in X \backslash \bar{A}$, então existe $j \in \omega$ tal que $d(x, y) \geq \frac{1}{j+1}$ para todo $y \in A$, e isso contraria a maximalidade de $A_{j}$.

Quando $\mathcal{B}$ é uma bornologia em $X$ com base compacta, podemos mostrar que a celularidade de $C_{\mathcal{B}}(X)$ está relacionada ao peso dos membros da bornologia $\mathcal{B}$, no seguinte sentido: definindo $w_{\mathcal{B}}(X):=\sup \{w(B): B \in \mathcal{B}\}$, mostra-se que $w_{\mathcal{B}}(X) \leq c\left(C_{\mathcal{B}}(X)\right)$; chamamos o cardinal $w_{\mathcal{B}}(X)$ de $\mathcal{B}$-peso do espaço $X$. Para provar tal desigualdade, precisamos do seguinte lema:

Lema 2.2.16 (Arhangel'skii, apud Hodel [21], Teorema 7.4). Se X é compacto Hausdorff, então $n w(X)=w(X)$.

Demonstração. (Engelking, [15], Teorema 3.1.19).

Já temos $n w(X) \leq w(X)$. Chamemos de $\tau$ a topologia de $X$. Suponha que exista uma topologia $\tau^{\prime}$ de Hausdorff em $X$ satisfazendo $\tau^{\prime} \subseteq \tau$ e $w\left(\left(X, \tau^{\prime}\right)\right) \leq n w((X, \tau))$. Assim,

$$
\begin{aligned}
f:(X, \tau) & \rightarrow\left(X, \tau^{\prime}\right) \\
x & \mapsto x
\end{aligned}
$$

é uma bijeção contínua de um compacto Hausdorff sobre um espaço de Hausdorff. Logo, $f$ é um homeomorfismo e, portanto, $w(X, \tau)=w\left(X, \tau^{\prime}\right) \leq n w(X, \tau)$. Vamos obter a topologia $\tau^{\prime}$.

Seja $\mathcal{N}$ uma network em $X$ com $|\mathcal{N}|=n w(X, \tau)$. Definamos $\mathcal{N}^{\prime}=\{(M, N) \in \mathcal{N} \times \mathcal{N}$ : $M \cap N=\emptyset\}$ e, para cada $(M, N) \in \mathcal{N}^{\prime}$, considere

$$
\mathcal{A}(M, N)=\{\{U, V\} \subseteq \tau: U \cap V=\emptyset, M \subseteq U \text { e } N \subseteq V\}
$$

Se $\mathcal{A}(M, N) \neq \emptyset$, escolha $\varphi(M, N) \in \mathcal{A}(M, N)$, caso contrário faça $\varphi(M, N)=\emptyset$. Vamos chamar de $\tau^{\prime}$ a topologia em $X$ gerada por $\mathfrak{B}=\bigcup\left\{\varphi(M, N):(M, N) \in \mathcal{N}^{\prime}\right\}$ como sub-base, donde já obtemos $w\left(X, \tau^{\prime}\right) \leq|\mathfrak{B}| \leq n w(X, \tau)$. Agora, como $\tau$ é de Hausdorff e $\mathcal{N}$ é uma network, segue que $\tau^{\prime}$ também é de Hausdorff. 
Observação 2.2.17. Em verdade, na demonstração acima mostramos que se $X$ é um espaço de Hausdorff, então existem um espaço de Hausdorff $Y$ e uma bijeção contínua $f: X \rightarrow Y$ tais que $w(Y) \leq n w(X)$.

Corolário 2.2.18. Se $\mathcal{B}$ tem base compacta, então $w_{\mathcal{B}}(X) \leq c\left(C_{\mathcal{B}}(X)\right)$.

Demonstração. Seja $B \in \mathcal{B}$ compacto (se necessário, podemos tomar $\bar{B}$, o qual é compacto, e ainda teremos $\left.w(B) \leq w(\bar{B}) \leq w_{\mathcal{B}}(X)\right)$. Como $\mathcal{B}$ é hereditária, segue que $\mathcal{B}^{\prime}=\{A \cap B: A \in$ $\mathcal{B}\}$ é uma bornologia em $B$, que tem $\{B\}$ como base compacta e, portanto, $a_{\mathcal{B}^{\prime}}(B)=\aleph_{0}$. Disso decorre a metrizabilidade de $C_{\mathcal{B}^{\prime}}(B)$ e, por conseguinte, $n w\left(C_{\mathcal{B}^{\prime}}(B)\right)=c\left(C_{\mathcal{B}^{\prime}}(B)\right)$ pelo Lema 2.2.15. Além disso, da compacidade de $B$, temos $n w(B)=w(B)$. Logo,

$$
w(B)=n w(B) \leq n w_{\mathcal{B}^{\prime}}(B)=n w\left(C_{\mathcal{B}^{\prime}}(B)\right)=c\left(C_{\mathcal{B}^{\prime}}(B)\right)
$$

Agora, defina $\varphi: C_{\mathcal{B}}(X) \rightarrow C_{\mathcal{B}^{\prime}}(B)$ por $\varphi(f)=f \uparrow B$. Como $B$ é compacto e $X$ é de Tychonoff, se $f \in C_{\mathcal{B}^{\prime}}(B)$, então existe uma extensão contínua $\tilde{f}: X \rightarrow \mathbb{R}$ para $f$ (cf. Corolário 1.2.2, o que atesta a sobrejetividade de $\varphi$. É fácil ver que $\varphi$ é contínua.

Dessa forma, $c\left(C_{\mathcal{B}^{\prime}}(B)\right) \leq c\left(C_{\mathcal{B}}(X)\right)$, pois se $f: Y \rightarrow Z$ é uma função contínua e sobre, então $c(Z) \leq c(Y)$ : basta ver que, se $\mathcal{U}$ é uma família de abertos não vazios dois a dois disjuntos de $Z$, então $\left\{f^{-1}[U]: U \in \mathcal{U}\right\}$ é uma família de abertos de $Y$ não vazios e dois-a-dois disjuntos. Portanto, $w(B) \leq c\left(C_{\mathcal{B}}(X)\right)$ para todo $B \in \mathcal{B}$, como queríamos.

Corolário 2.2.19 (McCoy e Ntantu, [31], Corolário 4.2.3). Se $C_{k}(X)$ é um espaço ccc, então todo compacto de X é metrizável.

Demonstração. Como $w_{\mathfrak{K}}(X)=\aleph_{0}$, segue que todo compacto $K$ de $X$ tem base enumerável e, como $X$ é de Tychonoff, concluímos que $K$ é metrizável.

Para um espaço de Tychonoff $X$ munido de uma bornologia $\mathcal{B}$ com base compacta, as desigualdades $c\left(C_{\mathcal{B}}(X)\right) \leq d\left(C_{\mathcal{B}}(X)\right) \leq w(X)$ acarretam que $C_{\mathcal{B}}(X)$ é um espaço ccc sempre que $w(X)=\aleph_{0}$ ou, mais geralmente, sempre que existir uma bijeção contínua $f: X \rightarrow Y$ com $Y$ de Tychonoff e $w(Y)=\aleph_{0}$.

A conclusão acima pode ser melhorada pelo seguinte resultado (Vidossich, apud Engelking [15], Exercício 4.4.C.b): se $X$ é um espaço de Tychonoff e existe uma bijeção contínua de $X$ 
sobre um espaço metrizável, então $C_{k}(X)$ é um espaço ccc. Daí, como $\mathcal{T}_{\mathcal{F}} \subseteq \mathcal{T}_{\mathcal{B}} \subseteq \mathcal{T}_{\mathfrak{K}}$ para toda bornologia $\mathcal{B}$ em $X$ com base compacta, temos $c\left(C_{p}(X)\right) \leq c\left(C_{\mathcal{B}}(X)\right) \leq c\left(C_{k}(X)\right)$ e assim, para tais espaços, $C_{\mathcal{B}}(X)$ é ccc. Chamando de submetrizável um espaço topológico $X$ para o qual existe uma bijeção contínua de $X$ sobre um espaço métrico, obtemos o seguinte corolário.

Corolário 2.2.20. Se X é um espaço de Tychonoff submetrizável, então $C_{\mathcal{B}}(X)$ é ccc para toda bornologia $\mathcal{B}$ em $X$ com base compacta. Em particular, se $X$ é metrizável, então $C_{\mathcal{B}}(X)$ é ccc.

Contudo, no contexto da $C_{p}$-teoria, $C_{p}(X)$ é um espaço ccc para qualquer espaço $X$. Tal fato segue do próximo resultado, que é corolário do Teorema de Hewitt-Marczewiski-Pondiczery:

Corolário 2.2.21 (Šanin, apud Engelking [15], Teorema 2.3.17). Se $\left\{X_{s}\right\}_{s \in S}$ é uma família de espaços topológicos com $d\left(X_{s}\right) \leq \kappa$ para todo $s \in S$, então $c\left(\prod_{s \in S} X_{s}\right) \leq \kappa$. Em particular, qualquer produto de espaços separáveis é um espaço ccc.

Demonstração. Suponha $c\left(\prod_{s \in S} X_{s}\right)>\kappa$, e tome $\mathcal{U}=\left\{U_{t}\right\}_{t \in T}$ uma família de abertos doisa-dois disjuntos de $\prod_{s \in S} X_{s}$, com $\kappa<|T| \leq 2^{\kappa}$. Podemos supor que cada $U_{t} \in \mathcal{U}$ é um aberto básico de $\prod_{s \in S} X_{s}$, donde segue que para cada $t \in T, \operatorname{supp}\left(U_{t}\right):=S_{t} \subseteq S$ é finito e, por conseguinte, $S^{\prime}=\bigcup_{t \in T} S_{t}$ tem cardinalidade $\leq 2^{\kappa}$. Logo, pelo Teorema de HewittMarczewiski-Pondiczery, existe $A \subseteq \prod_{s \in S^{\prime}} X_{s}$, tal que $|A| \leq \kappa$ e $A$ é denso em $\prod_{s \in S^{\prime}} X_{s}$. Restringindo os fatores de $U_{t}$ a $S^{\prime}$ para cada $t$, obtemos uma família $\mathcal{U}^{\prime}=\left\{U_{t}^{\prime}\right\}_{t \in T}$ de abertos dois-a-dois disjuntos em $\prod_{s \in S^{\prime}} X_{s}$, o que leva a uma contradição: por um lado, devemos ter $\left|\mathcal{U}^{\prime}\right|=|T|>\kappa$, contudo, cada membro de $\mathcal{U}^{\prime}$ contém ao menos um elemento de $A, \operatorname{logo}$ $|T| \leq|A| \leq \kappa$.

Teorema 2.2.22 (Arhangel'skii, apud Okuyama e Terada [35]). Para todo espaço $X, C_{p}(X) e ́$ um espaço ccc.

Demonstração (Tkachuk, [47], Problema 111). O corolário anterior nos dá $c\left(\mathbb{R}^{X}\right)=\aleph_{0}$, já que $\mathbb{R}$ é separável. Assim, levando em conta que $C_{p}(X)$ é denso em $\mathbb{R}^{X}$ ( $c f$. final da Seção 1.2), a demonstração do teorema se resume a verificar a seguinte afirmação, que deixamos a cargo do leitor: se $D$ é denso em $Y$, então $c(D)=c(Y)$. 


\subsection{Variações em tightness de $C_{\mathcal{B}}(X)$}

O tightness de um espaço topológico $X$ no ponto $x \in X$, indicado por $t(x, X)$, é o menor cardinal $\kappa \geq \aleph_{0}$ com a seguinte propriedade:

$$
\forall A \subseteq X\left(x \in \bar{A} \Rightarrow\left(\exists B \in[A]^{\leq \kappa}(x \in \bar{B})\right)\right)
$$

o tightness do espaço $X$ é o supremo de todos os cardinais $t(x, X)$ para $x \in X$.

Como a propriedade acima se verifica trivialmente para qualquer $\kappa$ quando $x \in A$, podemos considerar a família $\Omega_{x}$ de todos os subconjuntos de $X$ tais que $x \in \bar{A} \backslash A$ e, equivalentemente, definir $t(x, X)$ como sendo o menor cardinal $\kappa \geq \aleph_{0}$ tal que para todo $A \in \Omega_{x}$ existe $B \in$ $\Omega_{x} \cap[A] \leq \kappa$.

Assim, do mesmo modo que fizemos com o grau de Lindelöf $l(X)$, vamos considerar as variações seletivas ${ }^{10}$ do tightness, $\mathrm{S}_{1}^{\kappa}\left(\Omega_{x}, \Omega_{x}\right)$ e $\mathrm{S}_{f i n}^{\kappa}\left(\Omega_{x}, \Omega_{x}\right)$. É fácil ver que:

$$
\chi(x, X) \leq \kappa \Rightarrow \mathrm{S}_{1}^{\kappa}\left(\Omega_{x}, \Omega_{x}\right) \Rightarrow \mathrm{S}_{f i n}^{\kappa}\left(\Omega_{x}, \Omega_{x}\right) \Rightarrow t(x, X) \leq \kappa .
$$

Foquemo-nos no caso em que $\kappa=\aleph_{0}$. Se $\chi(x, X)=\aleph_{0}$, então para todo $A \subseteq X, x \in \bar{A}$ se, e somente se, existe uma sequência $\left(x_{n}\right)_{n \in \omega}$ de pontos de $A$ que converge para $x$. Novamente, observe que isto é trivial quando temos $x \in A$, de modo que, se chamarmos por $\Sigma_{x}:=\left\{\left\{x_{n}\right.\right.$ : $\left.n \in \omega\} \in[X]^{\leq \omega}: x_{n} \rightarrow x\right\}$, então a validade de $\chi(x, X)=\aleph_{0}$ implica que $X$ tem a seguinte propriedade:

$$
\forall A \in \wp(X)\left(A \in \Omega_{x} \Rightarrow\left(\exists B \in[A]^{\leq \omega} \cap \Sigma_{x}\right)\right)
$$

Dizemos que $X$ é um espaço de Frécher ${ }^{11}$ em $x \in X$ se $X$ satisfaz a condição 2.3.3 acima e, caso tal condição se verificar para todo $x \in X$, dizemos simplesmente que $X$ é um espaço de Fréchet. Seguindo a definição dada por Caserta et al. [10], diremos que $X$ é um espaço de Fréchet estrito em $x \in X$ (ou estritamente de Fréchet) se valer $\mathrm{S}_{1}\left(\Omega_{x}, \Sigma_{x}\right)$ e, se isso ocorrer para todo $x \in X, X$ será chamado de espaço de Fréchet estrito. É imediata a

\footnotetext{
${ }^{10}$ Usualmente na literatura, diz-se que $X$ tem fan tightness enumerável se valer $\mathrm{S}_{\text {fin }}\left(\Omega_{x}, \Omega_{x}\right)$; quando $X$ satisfaz $\mathrm{S}_{1}\left(\Omega_{x}, \Omega_{x}\right)$, diz-se que $X$ tem strong fan tightness enumerável.

${ }^{11}$ Em algumas referências também chamado de espaço de Fréchet-Urysohn.
} 
verificação das implicações

$$
\chi(x, X)=\aleph_{0} \Rightarrow \mathrm{S}_{1}\left(\Omega_{x}, \Sigma_{x}\right) \Rightarrow X \text { é espaço de Fréchet em } x \Rightarrow t(x, X)=\aleph_{0} .
$$

Nesta seção estudaremos as variações da função cardinal tightness que apresentamos acima, para espaços da forma $C_{\mathcal{B}}(X)$, onde $X$ é um espaço de Tychonoff munido de uma bornologia $\mathcal{B}$ com base compacta. Lembramos o leitor da observação (1) feita ao final da Seção 2.1, de que $C_{\mathcal{B}}(X)$ é um espaço homogêneo. Tal propriedade será fortemente usada nesta seção, pois a verificação de uma condição local num ponto base atesta a validade da mesma condição em todos os pontos do espaço - neste caso, usaremos como "ponto base" a função nula $\underline{0}$ : $X \rightarrow \mathbb{R}$. Veremos que em geral, propriedades de convergência em $C_{\mathcal{B}}(X)$ se traduzem como propriedades de recobrimento em $X$.

Ao longo desta seção, denotaremos por $I_{n}$ o intervalo aberto $]-\frac{1}{n+1}, \frac{1}{n+1}[$ para cada $n \in \omega$, e $\Omega_{\underline{0}}:=\left\{A \subset C_{\mathcal{B}}(X): \underline{0} \in \bar{A} \backslash A\right\}$.

\subsubsection{O tightness de $C_{\mathcal{B}}(X)$ e a propriedade de Fréchet}

Na seção anterior, vimos que $\chi\left(C_{\mathcal{B}}(X)\right)=a_{\mathcal{B}}(X)$, resultado que podemos considerar como o primeiro neste trabalho a relacionar uma propriedade de fechamento em $C_{\mathcal{B}}(X)$ com uma propriedade de recobrimento em $X$. Por um lado, decorre da definição de tightness que $t\left(C_{\mathcal{B}}(X)\right) \leq \chi\left(C_{\mathcal{B}}(X)\right)$ e, por outro lado, da definição de $\mathcal{B}$-coberturas, é fácil ver que $l_{\mathcal{B}}(X) \leq a_{\mathcal{B}}(X)$.

Assim, podemos nos perguntar se existe alguma relação entre $t\left(C_{\mathcal{B}}(X)\right)$ e $l_{\mathcal{B}}(X)$. Quando $\mathcal{B}$ tem base compacta, a resposta é afirmativa: ambos são iguais. O próximo lema, que intuitivamente permite "levar informações" de $\mathcal{O}_{\mathcal{B}}(X)$ para $\Omega_{\underline{0}}$ e vice-versa, é um resultado técnico que nos permitirá provar a igualdade proposta, bem como várias outras traduções de $C_{\mathcal{B}}(X)$ para $X$.

Lema 2.3.1 (Adaptado de Caserta et al., [10], Lema 2.2). Suponha que $\mathcal{B}$ tem base compacta em $X$.

(a) Para $\mathcal{U} \in \mathcal{O}_{\mathcal{B}}(X)$, chame $\mathcal{A}(\mathcal{U}):=\left\{f \in C_{\mathcal{B}}(X): \exists U \in \mathcal{U}(f \uparrow(X \backslash U) \equiv 1)\right\}$. Se U é não trivial, então $\mathcal{A}(\mathcal{U})$ é um elemento de $\Omega_{\underline{0}}$. 
(b) Sejam $A \subseteq C_{\mathcal{B}}(X), n \in \omega$ fixado e $\mathcal{U}_{n}(A):=\left\{f^{-1}\left[I_{n}\right]: f \in A\right\}$. Se $\underline{0} \in \bar{A}$, então $\mathcal{U}$ é $\mathcal{B}$-cobertura para $X$ (possivelmente trivial).

Demonstração.

(a) Claramente, temos $\underline{0} \notin \mathcal{A}(\mathcal{U})$. Para $B \in \mathcal{B}$ e $\varepsilon>0$, vamos obter $f \in \mathcal{A}(\mathcal{U})$ tal que $f \in\langle B, \varepsilon\rangle[\underline{0}]$. Por $\mathcal{U}$ ser $\mathcal{B}$-cobertura, existe $U \in \mathcal{U}$ que contém o compacto $\bar{B}$; note que como $X \notin \mathcal{U}$, temos $X \backslash U \neq \emptyset$. Pelo Lema de Urysohn "Fraco", existe uma função $f \in C_{\mathcal{B}}(X)$ satisfazendo $f \uparrow \bar{B} \equiv 0$ e $f \uparrow(X \backslash U) \equiv 1$, donde temos $f \in \mathcal{A}(\mathcal{U}) \cap\langle B, \varepsilon\rangle[\underline{0}]$. Portanto, $\underline{0} \in \overline{\mathcal{A}(\mathcal{U})} \backslash \mathcal{A}(\mathcal{U})$.

(b) Por termos $\underline{0} \in \bar{A}$, para $B \in \mathcal{B}$ qualquer obtemos $f \in A \cap\left\langle B, \frac{1}{n+1}\right\rangle[\underline{0}]$, i.e., existe $f \in A$ tal que $f[B] \subseteq I_{n}$ e, assim, $B \subseteq f^{-1}\left[I_{n}\right]$, o qual é aberto devido à continuidade de $f$. Logo, $\mathcal{U}_{n}(A)$ é uma $\mathcal{B}$-cobertura para $X$.

Note que se $f \in A$ é limitada, digamos que por $\frac{1}{n+1}$, então $\mathcal{U}_{n}(A)$ é uma $\mathcal{B}$-cobertura trivial, mesmo com $f(x) \neq 0$ para todo $x \in X$. Além disso, se $\mathcal{B}$ é a bornologia trivial, então o item (a) do lema anterior é válido por vacuidade: neste caso não existem $\mathcal{B}$-coberturas não triviais. Mais geralmente, $\mathcal{B}=\wp(X)$ implica em $\chi\left(C_{\mathcal{B}}(X)\right)=a_{\mathcal{B}}(X)=\aleph_{0}$, de modo que $C_{\mathcal{B}}(X)$ satisfaz trivialmente a condição de Fréchet $\left(\operatorname{logo}, C_{\mathcal{B}}(X)\right.$ tem tightness enumerável), bem como $X$ satisfaz $\mathrm{S}_{1}\left(\mathcal{O}_{\mathcal{B}}, \mathcal{O}_{\mathcal{B}}\right)$ (e, por conseguinte, $\left.l_{\mathcal{B}}(X)=\aleph_{0}\right)$. Por tal motivo, assumiremos que $\mathcal{B}$ é uma bornologia não trivial e com base compacta pelo restante desta seção.

Teorema 2.3.2 (McCoy e Ntantu, [31], Teorema 4.7.1). $t\left(C_{\mathcal{B}}(X)\right)=l_{\mathcal{B}}(X)$.

Demonstração. Considere $t\left(C_{\mathcal{B}}(X)\right)=\kappa$ e tome $\mathcal{U}$ uma $\mathcal{B}$-cobertura não trivial para $X$. Pelo item (a) do Lema 2.3.1, a família $\mathcal{A}(\mathcal{U})$ pertence a $\Omega_{\underline{0}}$, e a hipótese sobre o tightness nos permite obter $F=\left\{f_{\alpha}: \alpha<\kappa\right\} \subseteq \mathcal{A}(\mathcal{U}) \operatorname{com} F \in \Omega_{\underline{0}}$. Para cada $\alpha<\kappa$, escolha um aberto $U_{\alpha} \in \mathcal{U}$ que "ateste" $f_{\alpha} \in \mathcal{A}(\mathcal{U})$, i.e, $f_{\alpha} \uparrow\left(X \backslash U_{\alpha}\right) \equiv 1$. Afirmamos que $\mathcal{U}^{\prime}=\left\{U_{\alpha}: \alpha<\kappa\right\} \subseteq \mathcal{U}$ é uma $\mathcal{B}$-cobertura para $X$. Por $\underline{0} \in \bar{F}$, para $B \in \mathcal{B}$ qualquer existe $\alpha<\kappa$ tal que $f_{\alpha} \in\langle B, 1\rangle[\underline{0}]$ e, consequentemente, $B \subseteq U_{\alpha} \in \mathcal{U}^{\prime}$, caso contrário teríamos $f_{\alpha}(x)=1$ para algum $x \in B$ pela forma como tomamos $U_{\alpha}$. Temos assim $l_{\mathcal{B}}(X) \leq t\left(C_{\mathcal{B}}(X)\right)$. 
Assuma $l_{\mathcal{B}}(X)=\lambda$ e tome $A \subseteq C_{\mathcal{B}}(X)$ tal que $\underline{0} \in \bar{A}$. Para cada $n \in \omega$, vamos considerar $\mathcal{U}_{n}(A)$ como definido no item item (b) do Lema 2.3.1, donde segue que $\mathcal{U}_{n}(A) \in \mathcal{O}_{\mathcal{B}}$ para todo $n$. Logo, pela hipótese sobre $l_{\mathcal{B}}(X)$, para cada $n$ existe $A_{n} \in[A] \leq \lambda$ tal que $\mathcal{U}_{n}\left(A_{n}\right) \subseteq \mathcal{U}_{n}(A)$ e $\mathcal{U}_{n}\left(A_{n}\right)$ é uma $\mathcal{B}$-cobertura para $X$. Chamando $A^{\prime}=\bigcup_{n \in \omega} A_{n}$, temos $\left|A^{\prime}\right| \leq \lambda$; mostraremos que $\underline{0} \in \overline{A^{\prime}}$. De fato, para $B \in \mathcal{B}$ e $\varepsilon>0$ quaisquer, existe $n_{0} \in \omega \operatorname{com} \frac{1}{n_{0}+1}<\varepsilon$ e, por $\mathcal{U}_{n_{0}}\left(A_{n_{0}}\right)$ ser uma $\mathcal{B}$-cobertura para $X$, existe $g \in A_{n_{0}}$ tal que $B \subseteq g^{-1}\left[I_{n_{0}}\right]$, donde temos $g \in\langle B, \varepsilon\rangle[\underline{0}] \cap A^{\prime}$. Portanto, $t\left(C_{\mathcal{B}}(X)\right) \leq l_{\mathcal{B}}(X)$.

No contexto da $C_{k}$-teoria, o teorema acima nos dá $t\left(C_{k}(X)\right)=l_{\mathfrak{K}}(X)$ (McCoy, apud Kočinac [27]). Em particular, $C_{k}(X)$ tem tightness enumerável se, e somente se, toda $K$-cobertura de $X$ tem $K$-subcobertura enumerável. Para a $C_{p}$-teoria, o teorema acima se traduz na igualdade $t\left(C_{p}(X)\right)=l_{\mathcal{F}}(X)$ e, por meio do Teorema 2.1.3, obtemos o seguinte corolário:

Corolário 2.3.3 (Arhangel'skii, [4], Teorema II.1.1). Para um espaço de Tychonoff X, tem-se

$$
t\left(C_{p}(X)\right)=\sup \left\{l\left(X^{n}\right): n \in \omega\right\}
$$

Em particular, $t\left(C_{p}(X)\right)=\aleph_{0}$ se, e somente se, $X^{n}$ é um espaço de Lindelöf para todo $n \in \omega$.

Como $\mathbb{R}$ é um espaço de peso enumerável, inferimos que $\mathbb{R}^{n}$ tem peso enumerável para todo $n \in \omega$, o que implica em $l\left(\mathbb{R}^{n}\right)=\aleph_{0}$. Logo, pelo corolário acima, temos $t\left(C_{p}(\mathbb{R})\right)=\aleph_{0}$. Por outro lado, já que $|\mathbb{R}|>\aleph_{0}$, temos $\chi\left(C_{p}(\mathbb{R})\right)>\aleph_{0}$ e, por conseguinte, $C_{p}(\mathbb{R})$ não satisfaz o primeiro axioma de enumerabilidade.

No que concerne às propriedades de Fréchet e Fréchet estrito, veremos que ambas coincidem para espaços da forma $C_{\mathcal{B}}(X)$ e, para isso, precisaremos de uma nova classe de $\mathcal{B}$-coberturas em $X$. Adaptando as definições apresentadas em [9, 10, 14], uma $\mathcal{B}$-cobertura $\mathcal{U}$ infinita será chamada de $\gamma_{\mathcal{B}}$-cobertura para $X$ se para todo $B \in \mathcal{B}$ existir $F \in[\mathcal{U}]^{<\omega}$ tal que $B \subseteq U$ para todo $U \in \mathcal{U} \backslash F$; denotamos por $\Gamma_{\mathcal{B}}$ o conjunto de todas as $\gamma_{\mathcal{B}}$-coberturas abertas de $X$. Diremos que $X$ é um $\gamma_{\mathcal{B}}$-espaço se toda $\mathcal{B}$-cobertura não trivial de $X$ admitir uma $\gamma_{\mathcal{B}}$-subcobertura enumerável e, caso $X$ satisfaça $\mathrm{S}_{1}\left(\mathcal{O}_{\mathcal{B}}^{*}, \Gamma_{\mathcal{B}}\right)$, diremos que $X$ é um $\gamma_{\mathcal{B}}$-espaço estrito.

Lema 2.3.4. Se $\left(\mathcal{W}_{n}\right)_{n \in \omega}$ é uma sequência de $\mathcal{B}$-coberturas de $X$, então existe uma sequência $\left(\mathcal{U}_{n}\right)_{n \in \omega}$ de $\mathcal{B}$-coberturas de $X$ tal que para cada $n \in \omega, \mathcal{U}_{n+1}$ refina $\mathcal{W}_{m+1}$ e $\mathcal{U}_{m}$ para todo $m \leq n$. 
Demonstração. Chame $\mathcal{U}_{0}=\mathcal{W}_{0}$ e, para cada $n \in \omega$ com $n \geq 1$, defina $\mathcal{U}_{n+1}=\{U \cap W$ : $\left.U \in \mathcal{U}_{n} \wedge W \in \mathcal{W}_{n+1}\right\}$. Supondo que $\mathcal{U}_{m}$ seja $\mathcal{B}$-cobertura, provemos que $\mathcal{U}_{m+1}$ é também uma $\mathcal{B}$-cobertura de $X$. Para isso, tome $B \in \mathcal{B}$; como tanto $\mathcal{U}_{m}$ quanto $\mathcal{W}_{m+1}$ são $\mathcal{B}$-coberturas, segue que existem $U \in \mathcal{U}_{m}$ e $W \in \mathcal{W}_{m+1}$ tais que $B \subseteq U$ e $B \subseteq W$, donde inferimos que $B \subseteq U \cap W \in \mathcal{U}_{m+1}$. Assim, $\mathcal{U}_{m+1}$ é uma $\mathcal{B}$-cobertura para $X$. Por construção, $\mathcal{U}_{n+1}$ refina $\mathcal{W}_{n+1}$ e $\mathcal{U}_{n}$ e, por indução, $\mathcal{U}_{n+1}$ refina $\mathcal{W}_{m+1}$ e $\mathcal{U}_{m}$ para todo $m \leq n$.

Teorema 2.3.5 (McCoy e Ntantu, [31], Teorema 4.7.4; Caserta et al. [10], Corolário 2.10). São equivalentes:

(a) $C_{\mathcal{B}}(X)$ é um espaço de Fréchet estrito;

(b) $C_{\mathcal{B}}(X)$ é um espaço de Fréchet;

(c) X é um $\gamma_{\mathcal{B}}$-espaço;

(d) X é um $\gamma_{\mathcal{B}}$-espaço estrito.

Demonstração. Já vimos que $(a) \Rightarrow(b)$ em geral. Mostraremos que $(b) \Rightarrow(c) \Rightarrow(d) \Rightarrow(a)$.

$(b) \Rightarrow(c)$. Seja $\mathcal{U} \in \mathcal{O}_{\mathcal{B}}(X)$ não trivial. Pelo item (a) do Lema 2.3.1, temos $\mathcal{A}(\mathcal{U}) \in \Omega_{\underline{0}}$ e, pela hipótese $(b)$, existe $\left\{f_{n}: n \in \omega\right\} \subseteq \mathcal{A}(\mathcal{U})$ tal que $f_{n} \rightarrow_{\mathcal{T}_{\mathcal{B}}} \underline{0}$. Assim, para cada $n \in \omega$, basta tomar $U_{n} \in \mathcal{U}$ satisfazendo $f_{n} \uparrow X \backslash U_{n} \equiv 1$ e observar que $\mathcal{U}^{\prime}=\left\{U_{n}: n \in \omega\right\} \in \Gamma_{\mathcal{B}}$.

$(c) \Rightarrow(d)$. Seja $\left(\mathcal{W}_{n}\right)_{n \in \omega}$ uma sequência de $\mathcal{B}$-coberturas não triviais de $X$ e tome $\left(\mathcal{U}_{n}\right)_{n \in \omega}$ uma sequência de $\mathcal{B}$-coberturas (não triviais) como no lema anterior. Como a família $\mathcal{L}=$ $\{X \backslash\{x\}: x \in X\}$ é uma $\mathcal{B}$-cobertura não trivial de $X$, decorre da hipótese $(c)$ que existe uma sequência $\left(x_{n}\right)_{n \in \omega}$ de elementos de $X$ tal que $\mathcal{L}^{\prime}=\left\{X \backslash\left\{x_{n}\right\}: n \in \omega\right\}$ é uma $\gamma_{\mathcal{B}}$-cobertura de $X$; note que podemos assumir $x_{0}, x_{1}, \ldots$ dois a dois distintos.

Para cada $n \in \omega$, chame $\mathcal{U}_{n}^{\prime}=\left\{U \backslash\left\{x_{n}\right\}: U \in \mathcal{U}_{n}\right\}$ e considere $\mathcal{V}=\bigcup_{n \in \omega} \mathcal{U}_{n}^{\prime}$. Como no Lema 2.3.4, prova-se facilmente que $\mathcal{V}$ é uma $\mathcal{B}$-cobertura não trivial de $X$. Assim, novamente pela hipótese $(c)$, existe $\mathcal{V}^{\prime}=\left\{V_{n}: n \in \omega\right\} \subseteq \mathcal{V}$ tal que $\mathcal{V}^{\prime}$ é uma $\gamma_{\mathcal{B}}$-cobertura de $X$. Para cada $k \in \omega$, existe $n_{k} \in \omega$ satisfazendo $V_{k} \in \mathcal{U}_{n_{k}}^{\prime}$ e, por conseguinte, $V_{k}=U_{n_{k}} \backslash\left\{x_{n_{k}}\right\}$, com $U_{n_{k}} \in \mathcal{U}_{n_{k}}$. Contudo, por $\mathcal{U}_{n_{k}}$ refinar $\mathcal{W}_{n_{k}}$, existe $W_{n_{k}} \in \mathcal{W}_{n_{k}}$ tal que $V_{k} \subseteq U_{n_{k}} \subseteq W_{n_{k}}$. Note ainda que $\left\{n_{k}: k \in \omega\right\}$ é infinito pois, para $n \in \omega,\left\{x_{0}, \ldots, x_{n}\right\}=B \in \mathcal{B}$ e, como $\mathcal{V}^{\prime}$ é 
$\mathcal{B}$-cobertura, existe $k \in \omega \operatorname{com} B \subseteq V_{k}=U \backslash\left\{x_{n_{k}}\right\}$, acarretando $n_{k}>n$. Provemos que vale $\mathrm{S}_{1}\left(\mathcal{O}_{\mathcal{B}}^{*}, \Gamma_{\mathcal{B}}\right)$.

Fixe $k_{0}=0$ e, para cada $i \in \omega$, defina $k_{i+1}=\min \left\{k: k>k_{i}\right.$ e $\left.n_{k}>n_{k_{i}}\right\}$; note que tanto $\left(n_{k_{i}}\right)_{i \in \omega}$ quanto $\left(k_{i}\right)_{i \in \omega}$ são infinitas e crescentes. Seja $m \in \omega$.

- Se $m=n_{k_{i}}$ para algum $i$, faça $O_{m}=W_{n_{k_{i}}}$.

- Se $m<n_{k_{i}}$ para algum $i$, então $\mathcal{U}_{n_{k_{i}}}$ refina $\mathcal{W}_{m}$, donde segue que existe $W_{m}^{n_{k_{i}}} \in \mathcal{W}_{m}$ com $V_{k_{i}} \subseteq U_{n_{k_{i}}} \subseteq W_{m}^{n_{k_{i}}}$. Assim, se $m \neq n_{k_{i}}$ para todo $i$, faça $O_{m}=W_{m}^{n_{k_{i_{0}}}} \in \mathcal{W}_{m}$, onde $n_{k_{i_{0}}}$ é o menor $n_{k_{i}}$ tal que $m<n_{k_{i}}$.

Afirmamos enfim que $\left\{O_{n}: n \in \omega\right\} \in \Gamma_{\mathcal{B}}$. De fato, para $B \in \mathcal{B}$, existe $p \in \omega$ tal que $B \subseteq V_{k}$ para todo $k \geq p$. Seja então $k_{j_{0}} \in \omega \operatorname{com} k_{j_{0}} \geq p$ tal que $n_{k_{j}} \geq p$ se $k_{j} \geq k_{j_{0}}$. Se $m \geq n_{k_{j_{0}}}$, então há dois casos a considerar:

1. se $m=n_{k_{i}}$, então $k_{i} \geq k_{j_{0}} \geq p$ e $B \subseteq V_{k_{i}} \subseteq W_{n_{k_{i}}}=O_{m}$;

2. se $m \neq n_{k_{i}}$ para todo $i$, então $O_{m}=W_{m}^{n_{k_{i_{0}}}}$ e $n_{k_{i_{0}}}=\min \left\{n_{k_{i}}: m<n_{k_{i}}\right\} \geq n_{k_{j_{0}}}$, donde segue que $B \subseteq V_{k_{i_{0}}} \subseteq W_{m}^{n_{k_{i_{0}}}}=O_{m}$.

Mostramos assim que $\left\{O_{n}: n \in \omega\right\}$ é uma $\gamma_{\mathcal{B}}$-cobertura de $X$, com $O_{n} \in \mathcal{W}_{n}$, como queríamos.

$(d) \Rightarrow(a)$. Queremos provar que $C_{\mathcal{B}}(X)$ satisfaz $\mathrm{S}_{1}\left(\Omega_{\underline{0}}, \Sigma_{\underline{0}}\right)$. Para isso, tomamos $\left(A_{n}\right)_{n \in \omega}$ tal que $A_{n} \in \Omega_{\underline{0}}$ para todo $n \in \omega$, e fazemos $\mathcal{U}_{n}\left(A_{n}\right)$ como no Lema 2.3.1. Há essencialmente três casos a considerar.

Caso 1: $X \notin \mathcal{U}_{n}\left(A_{n}\right)$ para todo $n \in \omega$. Pelo item (b) do Lema 2.3.1, cada $\mathcal{U}_{n}\left(A_{n}\right)$ é uma $\mathcal{B}$-cobertura não trivial de $X$. Logo, pela hipótese $(d)$, para cada $n \in \omega$ existe uma função $f_{n} \in A_{n}$ tal que $\left\{f_{n}^{-1}\left[I_{n}\right]: n \in \omega\right\}$ é uma $\gamma_{\mathcal{B}}$-cobertura de $X$. É fácil ver que $f_{n} \rightarrow \mathcal{T}_{\mathcal{B}} \underline{0}$.

Caso 2: $X \notin \mathcal{U}_{n}\left(A_{n}\right)$ apenas para finitos $n \in \omega$. Neste caso, para cada $n \in \omega$, escolhemos $f_{n} \in A_{n}$ qualquer se $X \notin \mathcal{U}_{n}\left(A_{n}\right)$ e, quando $X \in \mathcal{U}_{n}\left(A_{n}\right)$, escolhemos $f_{n} \in A_{n}$ tal que $f_{n}^{-1}\left[I_{n}\right]=X$, donde segue que $f_{n} \rightarrow \mathcal{T}_{\mathcal{B}} \underline{0}$.

Caso 3: Cada um dos casos $X \in \mathcal{U}_{n}\left(A_{n}\right)$ e $X \notin \mathcal{U}_{n}\left(A_{n}\right)$ ocorre para infinitos $n \in \omega$. Basta usarmos o Caso 1 para a sequência $\operatorname{dos} \mathcal{U}_{n}\left(A_{n}\right)$ não triviais e o Caso 2 para a sequência dos $\mathcal{U}_{n}\left(A_{n}\right)$ triviais. 
Vimos anteriormente que $C_{p}(\mathbb{R})$ é um espaço de tightness enumerável que não satisfaz o primeiro axioma de enumerabilidade. Mostraremos que este mesmo espaço também não é de Fréchet, o que pelo teorema provado acima, atesta a falsidade das recíprocas de (2.3.4). Para $F \subseteq \mathbb{R}$ e $n \in \omega$ fixado chame de

$$
B_{F}^{n}=\bigcup_{x \in F} B\left(x, \frac{1}{(|F|+1) 2^{n+1}}\right)
$$

e defina $\mathcal{U}_{n}=\left\{B_{F}^{n}: F \in[\mathbb{R}]^{<\omega}\right\}$, que claramente é uma $\omega$-cobertura aberta para $\mathbb{R}$. Seja $B_{F_{n}}^{n} \in \mathcal{U}_{n}$ para cada $n$ e faça $\mathcal{U}=\left\{B_{F_{n}}^{n}: n \in \omega\right\}$. Afirmamos que $\mathcal{U}$ não cobre $\mathbb{R}$. De fato, se $\mu$ denota a medida de Lebesgue de $\mathbb{R}$, observe que

$$
\mu(\bigcup \mathcal{U}) \leq \sum_{n=0}^{\infty} \mu\left(B_{F_{n}}^{n}\right) \leq \sum_{n=0}^{\infty}\left|F_{n}\right| \cdot \frac{1}{\left(\left|F_{n}\right|+1\right) \cdot 2^{n+1}} \leq 1
$$

enquanto $\mu(\mathbb{R})=+\infty$. A posteriori, o mesmo argumento apresentado acima mostra que $C_{p}(\mathbb{R})$ é contraexemplo para as recíprocas de $\chi(y, Y)=\aleph_{0} \Rightarrow \mathrm{S}_{1}\left(\Omega_{y}, \Omega_{y}\right) \Rightarrow t(y, Y)=\aleph_{0}$.

\subsubsection{Variações seletivas de tightness em $C_{\mathcal{B}}(X)$}

Na subseção anterior, mostramos que o tightness de $C_{\mathcal{B}}(X)$ coincide com o grau de $\mathcal{B}$ Lindelöf de $X$. Essa relação se mantém válida quando analisamos as variações seletivas de tais propriedades, assunto do qual trata esta subseção. Começamos com o seguinte teorema:

Teorema 2.3.6 (Baseado nos Teoremas 2.3 e 2.5 de Caserta et al. [10]).

(a) $C_{\mathcal{B}}(X)$ satisfaz $\mathrm{S}_{\text {fin }}\left(\Omega_{\underline{0}}, \Omega_{\underline{0}}\right)$ se, e somente se, $X$ satisfaz $\mathrm{S}_{\text {fin }}\left(\mathcal{O}_{\mathcal{B}}, \mathcal{O}_{\mathcal{B}}\right)$.

(b) $C_{\mathcal{B}}(X)$ satisfaz $\mathrm{S}_{1}\left(\Omega_{\underline{0}}, \Omega_{\underline{0}}\right)$ se, e somente se, $X$ satisfaz $\mathrm{S}_{1}\left(\mathcal{O}_{\mathcal{B}}, \mathcal{O}_{\mathcal{B}}\right)$.

\section{Demonstração.}

$(a)(\Rightarrow)$. Suponha que $X$ satisfaça $\mathrm{S}_{\text {fin }}\left(\mathcal{O}_{\mathcal{B}}, \mathcal{O}_{\mathcal{B}}\right)$ e tome $\left(A_{n}\right)_{n \in \omega} \operatorname{com} A_{n} \in \Omega_{\underline{0}}$ em $C_{\mathcal{B}}(X)$. Fazendo $\mathcal{U}_{n}\left(A_{n}\right)$ como no Lema 2.3.1, para todo $n$ temos $\mathcal{U}_{n}\left(A_{n}\right) \in \mathcal{O}_{\mathcal{B}}$. Caso $X \in \mathcal{U}_{n}\left(A_{n}\right)$ para infinitos $n$, tome $f_{n} \in A_{n}$ para cada $n$ atestando $X \in \mathcal{U}_{n}\left(A_{n}\right)$ e note que $\left\{f_{n}: n \in\right.$ $\omega\} \in \Omega_{\underline{0}}$, donde segue que $C_{\mathcal{B}}(X)$ satisfaz $\mathrm{S}_{1}\left(\Omega_{\underline{0}}, \Omega_{\underline{0}}\right)$ e, por conseguinte, satisfaz $\mathrm{S}_{f i n}\left(\Omega_{\underline{0}}, \Omega_{\underline{0}}\right)$. 
Desse modo, podemos assumir $X \notin \mathcal{U}_{n}\left(A_{n}\right)$ para todo $n$ e, assim, $\mathcal{U}_{n}\left(A_{n}\right)$ é uma $\mathcal{B}$-cobertura não trivial de $X$.

Pela hipótese de que $X$ satisfaz $\mathrm{S}_{f i n}\left(\mathcal{O}_{\mathcal{B}}, \mathcal{O}_{\mathcal{B}}\right)$, existe uma sequência $\left(F_{n}\right)_{n \in \omega}$, com $F_{n} \in$ $\left[A_{n}\right]^{<\omega}$, tal que $\bigcup_{n \in \omega} \mathcal{U}_{n}\left(F_{n}\right)$ é uma $\mathcal{B}$-cobertura para $X$ (que é não trivial). Afirmamos que $\bigcup_{n \in \omega} F_{n} \in \Omega_{\underline{0}}$. De fato, para uma vizinhança $\langle B, \varepsilon\rangle[\underline{0}]$, fixe $n \in \omega \operatorname{com} \frac{1}{n+1}<\varepsilon$ e note que, pelo Lema 2.1.1 $\left.\right|^{2}, \bigcup_{m \geq n} \mathcal{U}_{m}\left(F_{m}\right) \in \mathcal{O}_{\mathcal{B}}$. Assim, existe $m \geq n$ tal que $B \subseteq f^{-1}\left[I_{m}\right]$ para alguma $f \in F_{m}$, donde temos $\bigcup_{n \in \omega} F_{n} \in \Omega_{\underline{0}}$.

$(a)(\Leftarrow)$. Assuma que $C_{\mathcal{B}}(X)$ satisfaz $\mathrm{S}_{\text {fin }}\left(\Omega_{\underline{0}}, \Omega_{\underline{0}}\right)$, e seja $\left(\mathcal{U}_{n}\right)_{n \in \omega}$ uma sequência de $\mathcal{B}$ coberturas de $X$, que sem perda de generalidade podemos supor não triviais. Novamente o Lema 2.3 .1 nos garante que $\mathcal{A}\left(\mathcal{U}_{n}\right) \in \Omega_{\underline{0}}$ para cada $n \in \omega$. Como vale $\mathrm{S}_{\text {fin }}\left(\Omega_{\underline{0}}, \Omega_{\underline{0}}\right)$, podemos obter uma sequência $\left(F_{n}\right)_{n \in \omega}, \operatorname{com} F_{n} \in\left[\mathcal{A}\left(\mathcal{U}_{n}\right)\right]^{<\omega}$, tal que $\bigcup_{n \in \omega} F_{n} \in \Omega_{\underline{0}}$. Para cada $n$, escolha $V_{n} \in\left[\mathcal{U}_{n}\right]^{<\omega}$ atestando $F_{n} \in\left[\mathcal{A}\left(\mathcal{U}_{n}\right)\right]^{<\omega}$. É fácil ver que $\bigcup_{n \in \omega} V_{n} \in \mathcal{O}_{\mathcal{B}}$.

Os mesmos argumentos utilizados acima provam $(b)$, mutatis mutandis.

Em particular, chamando de $\Omega_{\underline{0}_{n}}=\left\{A \subset C_{\mathcal{B}^{n}}\left(X^{n}\right): \underline{0}_{n} \in \bar{A} \backslash A\right\}$, onde $\underline{0}_{n}: X^{n} \rightarrow \mathbb{R}$ é a função nula, segue do teorema acima e do Teorema 2.1 .4 que

$$
C_{\mathcal{B}}(X) \text { satisfaz } \mathrm{S}_{1}\left(\Omega_{\underline{0}}, \Omega_{\underline{0}}\right) \Leftrightarrow C_{\mathcal{B}^{n}}\left(X^{n}\right) \text { satisfaz } \mathrm{S}_{1}\left(\Omega_{\underline{0}_{n}}^{n}, \Omega_{\underline{0}_{n}}^{n}\right) \text { para todo } n \in \omega
$$

bem como a equivalência análoga para a variação $S_{f i n}$.

O teorema anterior se traduz nos seguintes corolários para o contexto da $C_{k}$-teoria.

Corolário 2.3.7 (Shou Lin et al., apud Kočinac [27]). $C_{k}(X)$ satisfaz $\mathrm{S}_{f i n}\left(\Omega_{\underline{0}}, \Omega_{\underline{0}}\right)$ se, e somente se, $X$ satisfaz $\mathrm{S}_{\text {fin }}(\mathcal{K}, \mathcal{K})$.

Corolário 2.3.8 (Kočinac, [27], Teorema 2.2). $C_{k}(X)$ satisfaz $\mathrm{S}_{1}\left(\Omega_{\underline{0}}, \Omega_{\underline{0}}\right)$ se, e somente se, $X$ satisfaz $\mathrm{S}_{1}(\mathcal{K}, \mathcal{K})$.

Ao fazermos $\mathcal{B}=\mathcal{F}$ no teorema acima, obtemos por meio do Teorema 2.1.6 as seguintes traduções para a topologia da convergência pontual:

Corolário 2.3.9 (Sakai, [38]). São equivalentes:

\footnotetext{
${ }^{12}$ Aqui utilizamos a suposição de não trivialidade das $\mathcal{B}$-coberturas.
} 
(a) $X$ satisfaz $\mathrm{S}_{1}(\Omega, \Omega)$;

(b) $X^{m}$ é de Rothberger para todo $m \in \omega$;

(c) $C_{p}(X)$ satisfaz $\mathrm{S}_{1}\left(\Omega_{\underline{0}}, \Omega_{\underline{0}}\right)$.

Corolário 2.3.10 (Arhangel'skii ${ }^{13}$, [4], Teorema II.2.2). São equivalentes:

(a) X satisfaz $\mathrm{S}_{f i n}(\Omega, \Omega)$;

(b) $X^{m}$ é de Menger para todo $m \in \omega$;

(c) $C_{p}(X)$ satisfaz $\mathrm{S}_{f i n}\left(\Omega_{\underline{0}}, \Omega_{\underline{0}}\right)$.

No que segue, a próxima notação será bastante útil. Para $\gamma$ e $\eta$ ordinais, denotaremos por

$$
\left(A_{\alpha}: \alpha<\gamma\right)^{\frown}\left(B_{\xi}: \xi<\eta\right):=\left(C_{\alpha}: \alpha<\gamma+\eta\right)
$$

a sequência de comprimento $\gamma+\eta$, onde $C_{\alpha}=A_{\alpha}$ se $\alpha<\gamma$, e $C_{\gamma+\xi}=B_{\xi}$ se $\xi<\eta$. Analisaremos as variações via jogos seletivos das propriedades estudadas acima, no que concerne à existência de estratégias vencedoras para os jogadores I e II nos jogos do tipo $\mathrm{G}_{1}$ e $\mathrm{G}_{\text {fin }}$. A princípio, vamos nos preocupar com a situação do jogador II.

Observe que se o jogador II tem estratégia vencedora em $\mathrm{G}_{1}^{\alpha}\left(\mathcal{O}_{\mathcal{B}}, \mathcal{O}_{\mathcal{B}}\right)$ para algum ordinal $\alpha \geq \omega$, então para qualquer ordinal $\beta \geq \alpha$ existe uma estratégia vencedora para o jogador II em $\mathrm{G}_{1}^{\beta}\left(\mathcal{O}_{\mathcal{B}}, \mathcal{O}_{\mathcal{B}}\right)$ : intuitivamente, o jogador II sabe como vencer jogando por apenas $\alpha$ turnos, de modo que nas demais rodadas ele pode jogar "aleatoriamente". É claro que a mesma observação é válida para os jogos da forma $\mathrm{G}_{f i n}$.

Note que existe um ordinal $\alpha$ para o qual II $\uparrow \mathrm{G}_{1}^{\alpha}\left(\mathcal{O}_{\mathcal{B}}, \mathcal{O}_{\mathcal{B}}\right)$ e II $\uparrow \mathrm{G}_{f i n}^{\alpha}\left(\mathcal{O}_{\mathcal{B}}, \mathcal{O}_{\mathcal{B}}\right)$ : basta tomar, por exemplo, $\alpha=|\mathcal{B}|$ pois, se $\mathcal{B}=\left\{B_{\gamma}: \gamma<\alpha\right\}$, então no $\gamma$-ésimo turno do jogo, o jogador II responde ao lance $\mathcal{U}_{\gamma} \in \mathcal{O}_{\mathcal{B}}$ do jogador I escolhendo $U \in \mathcal{U}_{\gamma}$ tal que $B_{\gamma} \subseteq U$.

Assim, adaptando a definição dada por Scheepers em [41], podemos considerar os invariantes topológicos

$$
\operatorname{tp}_{1}\left(\mathcal{O}_{\mathcal{B}}(X)\right):=\min \left\{\alpha: \text { II } \uparrow \mathrm{G}_{1}^{\alpha}\left(\mathcal{O}_{\mathcal{B}}(X), \mathcal{O}_{\mathcal{B}}(X)\right)\right\}
$$

\footnotetext{
${ }^{13} \operatorname{Para}(b) \Leftrightarrow(c)$.
} 
$\mathrm{e}$

$$
\operatorname{tp}_{f i n}\left(\mathcal{O}_{\mathcal{B}}(X)\right):=\min \left\{\alpha: \text { II } \uparrow \mathrm{G}_{f i n}^{\alpha}\left(\mathcal{O}_{\mathcal{B}}(X), \mathcal{O}_{\mathcal{B}}(X)\right)\right\}
$$

Veremos que tais ordinais são aditivamente indecomponíveis, o que permitirá obter estratégias vencedoras para o jogador II com propriedades adicionais. Dizemos que um ordinal $\alpha$ é aditivamente indecomponível (indecomposable ordinal) se para quaisquer $\gamma, \delta<\alpha$ valer $\gamma+\delta<\alpha$. Os ordinais 0 e $\omega$ são os exemplos triviais de ordinais aditivamente indecomponíveis; no Capítulo 9 de [28], os autores apresentam uma demonstração detalhada de que os ordinais com tal propriedade são exatamente aqueles da forma $\omega^{\alpha}$, para todo ordinal $\alpha$ (somente aqui, tal notação indica exponenciação de ordinais); em particular, se $A \cup B=\alpha$, onde $\alpha$ é aditivamente indecomponível, então $A$ ou $B$ tem tipo de ordem $\alpha$.

Lema 2.3.11. Se um ordinal $\alpha \geq \omega$ é aditivamente indecomponível, então existe uma família $Y=\left\{Y_{i}: i<\operatorname{cof}(\alpha)\right\}$ satisfazendo:

(a) Y é uma família de subconjuntos de $\alpha$ dois-a-dois disjuntos;

(b) para cada $i<\operatorname{cof}(\alpha)$, $Y_{i}$ tem tipo de ordem $\alpha$;

(c) $\alpha=\bigcup Y$;

(d) $\left\{\min Y_{i}: i<\operatorname{cof}(\alpha)\right\}$ é cofinal em $\alpha$.

Em [41], Scheepers enuncia (sem demonstrar) o lema acima, e o utiliza em [40] para relacionar estratégias para o jogador II no jogo $\mathrm{G}_{1}^{\alpha}(\Omega, \Omega)$ em $X$ e no jogo $\mathrm{G}_{1}^{\alpha}\left(\Omega_{\underline{0}}, \Omega_{\underline{0}}\right)$ em $C_{p}(X)$. Tal recurso técnico é utilizado para obter um tipo de generalização do Lema 2.1.1 - para o caso em que $\alpha=\omega$, tanto o lema acima quanto o próximo não são necessários ( $c f$. Observação 2.3.14). Como utilizaremos tal resultado somente para ordinais enumeráveis (Teorema 2.3.13), vamos apresentar o esboço de uma demonstração para o Lema acima válida para esta classe de ordinais:

Esboço da Demonstração do Lema 2.3.11. Para um ordinal aditivamente indecomponível $\alpha$ enumerável, temos $\operatorname{cof}(\alpha)=\aleph_{0}$. Seja $f: \alpha \rightarrow \alpha$ uma função injetora que preserva ordem tal que $f(0)>0$ e $\alpha \backslash f[\alpha]$ tenha tipo de ordem $\alpha$. Defina $Y_{0}=\alpha \backslash f[\alpha]$ e, chamando por $f^{n+1}=f \circ f^{n}$ 
para cada $n \in \omega$, defina $Y_{n+1}=\alpha \backslash\left(f^{n+1}[\alpha] \cup \bigcup_{j \leq n} Y_{j}\right)$ (a condição $f(0)>0$ garante que os mínimos de $Y_{i}$ sejam cofinais em $\alpha$ ).

Teorema 2.3.12 (Adaptado do Teorema 4 [41] e das Proposições 17 e 19 [40] de Scheepers). Sejam X um espaço topológico (não necessariamente de Tychonoff) e $\mathcal{B}$ uma bornologia em $X$ (não necessariamente com base compacta).

(a) $\operatorname{tp}_{1}\left(\mathcal{O}_{\mathcal{B}}(X)\right)$ é aditivamente indecomponível.

(b) $\operatorname{tp}_{f i n}\left(\mathcal{O}_{\mathcal{B}}(X)\right)$ é aditivamente indecomponível.

(c) Se $\operatorname{tp}_{1}\left(\mathcal{O}_{\mathcal{B}}(X)\right)=\alpha$, então existe uma estratégia vencedora $\rho$ para o jogador II em $\mathrm{G}_{1}^{\alpha}\left(\mathcal{O}_{\mathcal{B}}, \mathcal{O}_{\mathcal{B}}\right)$ com a seguinte propriedade: se $\left(\left(\mathcal{U}_{\gamma}, U_{\gamma}\right): \gamma<\alpha\right)$ é uma partida jogada em $\rho$, então para todo $\gamma<\alpha$ temos $\left\{U_{\xi}: \gamma<\xi<\alpha\right\} \in \mathcal{O}_{\mathcal{B}}$.

(d) Se $\operatorname{tp}_{f i n}\left(\mathcal{O}_{\mathcal{B}}(X)\right)=\alpha$, então existe uma estratégia vencedora $\rho$ para o jogador II em $\mathrm{G}_{\text {fin }}^{\alpha}\left(\mathcal{O}_{\mathcal{B}}, \mathcal{O}_{\mathcal{B}}\right)$ com a seguinte propriedade: se $\left(\left(\mathcal{U}_{\gamma}, V_{\gamma}\right): \gamma<\alpha\right)$ é uma partida jogada em $\rho$, então para todo $\gamma<\alpha$ temos $\bigcup_{\gamma<\xi<\alpha} V_{\xi} \in \mathcal{O}_{\mathcal{B}}$.

Demonstração.

(a) Sejam $\gamma, \delta<\operatorname{tp}_{1}\left(\mathcal{O}_{\mathcal{B}}(X)\right)$ e $\Phi$ uma estratégia para o jogador II em $\mathrm{G}_{1}^{\gamma+\delta}\left(\mathcal{O}_{\mathcal{B}}, \mathcal{O}_{\mathcal{B}}\right)$. Claramente, $\Phi$ induz uma estratégia $\mu$ para o jogador II em $\mathrm{G}_{1}^{\gamma}\left(\mathcal{O}_{\mathcal{B}}, \mathcal{O}_{\mathcal{B}}\right)$, por meio da restrição do domínio de $\Phi$. Como $\gamma<\operatorname{tp}_{1}\left(\mathcal{O}_{\mathcal{B}}(X)\right)$, segue que $\mu$ não é vencedora para o jogador II em $\mathrm{G}_{1}^{\gamma}\left(\mathcal{O}_{\mathcal{B}}, \mathcal{O}_{\mathcal{B}}\right)$. Logo, existe uma partida $P_{0}=\left(\left(\mathcal{V}_{\xi}, V_{\xi}\right): \xi<\gamma\right)$ jogada em $\mu$ e vencida pelo jogador I, isto é, tal que $\mathcal{V}_{0}=\left\{V_{\xi}: \xi<\gamma\right\} \notin \mathcal{O}_{\mathcal{B}}$. Isso nos permite obter $B_{0} \in \mathcal{B}$ que não é coberto por qualquer elemento de $\mathcal{V}_{0}$.

Definamos então uma estratégia $\sigma$ para o jogador II em $\mathrm{G}_{1}^{\delta}\left(\mathcal{O}_{\mathcal{B}}, \mathcal{O}_{\mathcal{B}}\right)$ da seguinte maneira: para uma sequência $\left(\mathcal{U}_{\eta}: \eta \leq \beta\right) \in\left(\mathcal{O}_{\mathcal{B}}\right)^{<\delta}, \sigma\left(\left(\mathcal{U}_{\eta}: \eta \leq \beta\right)\right)=\Phi\left(\left(\mathcal{V}_{\xi}: \xi<\gamma\right)^{\frown}\left(\mathcal{U}_{\eta}: \eta \leq \beta\right)\right)$, que está bem definido pois $\left(\mathcal{V}_{\xi}: \xi<\gamma\right)^{\frown}\left(\mathcal{U}_{\eta}: \eta \leq \beta\right) \in\left(\mathcal{O}_{\mathcal{B}}\right)^{<\gamma+\delta}$. Como $\delta<\operatorname{tp}_{1}\left(\mathcal{O}_{\mathcal{B}}(X)\right)$, decorre que $\sigma$ não é vencedora para o jogador II e, por conseguinte, existe uma partida $P_{1}=$ $\left(\left(\mathcal{V}_{\nu}^{\prime}, V_{\nu}^{\prime}\right): \nu<\delta\right) \operatorname{com} \mathcal{V}_{1}=\left\{V_{\nu}^{\prime}: \nu<\delta\right\} \notin \mathcal{O}_{\mathcal{B}}$ e, assim, existe $B_{1} \in \mathcal{B}$ que não é coberto por qualquer elemento de $\mathcal{V}_{1}$. 
Note então que $\left(\left(\mathcal{W}_{\beta}, W_{\beta}\right): \beta<\gamma+\delta\right)=P_{0} P_{1}$ é uma partida jogada em $\Phi$ que é vencida pelo jogador I, pois nenhum aberto de $\mathcal{V}=\left\{W_{\beta}: \beta<\gamma+\delta\right\}=\mathcal{V}_{0} \cup \mathcal{V}_{1}$ recobre $B_{0} \cup B_{1} \in \mathcal{B}$. Logo, $\gamma+\delta<\operatorname{tp}_{1}\left(\mathcal{O}_{\mathcal{B}}(X)\right)$. De maneira inteiramente análoga se prova a validade de $(b)$.

(c). Pela parte $(a)$, segue que $\operatorname{tp}_{1}\left(\mathcal{O}_{\mathcal{B}}(X)\right)=\alpha$ é aditivamente indecomponível. Isso nos permite obter uma família $Y$ como no Lema 2.3.11. Assim, se $\mu$ é uma estratégia vencedora para o jogador II em $\mathrm{G}_{1}^{\alpha}\left(\mathcal{O}_{\mathcal{B}}(X), \mathcal{O}_{\mathcal{B}}(X)\right)$, defina $\rho$ da seguinte maneira: para $\left(\mathcal{U}_{\gamma}: \gamma \leq \beta\right) \in$ $\left(\mathcal{O}_{\mathcal{B}}\right)^{<\alpha}$,

$$
\rho\left(\left(\mathcal{U}_{\gamma}: \gamma \leq \beta\right)\right)=\mu\left(\left(\mathcal{U}_{\gamma}: \gamma \leq \beta \wedge \gamma \in Y_{i}\right)\right) \text { se } \beta \in Y_{i}
$$

note que $\rho$ está bem definida pois os $Y_{i}$ 's são dois-a-dois disjuntos. Afirmamos que $\rho$ satisfaz a condição imposta. De fato, seja uma partida $\left(\left(\mathcal{U}_{\xi}, U_{\xi}\right): \xi<\alpha\right)$ jogada em $\rho$ e fixe $\gamma<\alpha$. Escolha $i<\operatorname{cof}(\alpha) \operatorname{com} \gamma<\min Y_{i}$. Assim, $Y_{i} \subset(\gamma, \alpha)$ e, como $Y_{i}$ tem tipo de ordem $\alpha$ e $\rho$ essencialmente restringe $\mu$ a cada partição $Y_{i}$, segue que $\left(\left(\mathcal{U}_{\xi}, U_{\xi}\right): \xi \in Y_{i}\right)$ é uma partida jogada em $\mu$, donde inferimos que $\left\{U_{\xi}: \xi \in Y_{i}\right\}$ é uma $\mathcal{B}$-cobertura para $X$ e, por conseguinte, $\left\{U_{\xi}: \gamma<\xi<\alpha\right\}$ também é uma $\mathcal{B}$-cobertura, como queríamos. A argumentação para $(d)$ pode ser feita de maneira análoga.

Teorema 2.3.13. Seja $\alpha \geq \omega$ um ordinal enumerável.

(a) II $\uparrow \mathrm{G}_{1}^{\alpha}\left(\Omega_{\underline{0}}, \Omega_{\underline{0}}\right)$ se, e somente se, II $\uparrow \mathrm{G}_{1}^{\alpha}\left(\mathcal{O}_{\mathcal{B}}, \mathcal{O}_{\mathcal{B}}\right)$.

(b) II $\uparrow \mathrm{G}_{\text {fin }}^{\alpha}\left(\Omega_{\underline{0}}, \Omega_{\underline{0}}\right)$ se, e somente se, II $\uparrow \mathrm{G}_{\text {fin }}^{\alpha}\left(\mathcal{O}_{\mathcal{B}}, \mathcal{O}_{\mathcal{B}}\right)$.

Demonstração. Dada a similaridade entre $(a)$ e $(b)$, provaremos apenas $(a)$.

Suponha que exista estratégia vencedora para o jogador II no jogo $\mathrm{G}_{1}^{\alpha}\left(\mathcal{O}_{\mathcal{B}}, \mathcal{O}_{\mathcal{B}}\right)$. Vamos mostrar que o jogador II tem estratégia vencedora no jogo $\mathrm{G}_{1}^{\lambda}\left(\Omega_{\underline{0}}, \Omega_{\underline{0}}\right)$, onde $\lambda=\operatorname{tp}_{1}\left(\mathcal{O}_{\mathcal{B}}(X)\right) \leq$ $\alpha$. Disto seguirá que o jogador II tem estratégia vencedora no jogo $\mathrm{G}_{1}^{\alpha}\left(\Omega_{\underline{0}}, \Omega_{\underline{0}}\right)$ - basta que o jogador II jogue "aleatoriamente" nas rodadas $>\lambda$, como já observamos.

Seja $\Phi$ uma estratégia vencedora para o jogador II em $\mathrm{G}_{1}^{\lambda}\left(\mathcal{O}_{\mathcal{B}}, \mathcal{O}_{\mathcal{B}}\right)$, e suponha que $\Phi$ tenha a propriedade $(c)$ do teorema anterior. Como $\alpha$ é enumerável, temos $\lambda$ enumerável e, por conseguinte, $\operatorname{cof}(\lambda) \leq \omega$, e então existe uma sequência $\left(\xi_{n}\right)_{n \in \omega}$ estritamente crescente e cofinal em $\lambda$. Para cada $\beta<\lambda$ associamos o número $n_{\beta} \in \omega$, onde $n_{\beta}=\min \left\{m: \xi_{m} \leq \beta<\xi_{m+1}\right\}$. 
Para quaisquer $n \in \omega$ e $A \in \Omega_{\underline{0}}$ consideramos a família $\mathcal{U}_{n}(A)$ como no Lema 2.3.1. que é uma $\mathcal{B}$-cobertura para $X$ (possivelmente trivial).

Vamos definir uma estratégia $\eta$ para o jogador II no jogo $\mathrm{G}_{1}^{\lambda}\left(\Omega_{\underline{0}}, \Omega_{\underline{0}}\right)$ em $C_{\mathcal{B}}(X)$ da seguinte maneira: para uma sequência $\left(A_{\gamma}: \gamma \leq \beta\right) \in \Omega_{\underline{0}}^{<\lambda}$, fazemos $\eta\left(\left(A_{\gamma}: \gamma \leq \beta\right)\right)=f \in A_{\beta}$, onde $f$ satisfaz:

$$
f^{-1}\left[I_{n_{\beta}}\right]=\Phi\left(\left(\mathcal{U}_{n_{\gamma}}\left(A_{\gamma}\right): \gamma \leq \beta\right)\right)
$$

O Lema 2.3.1 garante que $\eta$ está bem definida. Veremos que $\eta$ é vencedora.

Seja uma partida $P=\left(\left(A_{\beta}, f_{\beta}\right): \beta<\lambda\right)$ jogada em $\eta$ e chame $U_{\beta}=f_{\beta}^{-1}\left[I_{n_{\beta}}\right]$ para cada $\beta<\lambda$. Pelo modo como tomamos $\eta$, segue que $\left(\left(\mathcal{U}_{n_{\beta}}\left(A_{\beta}\right), U_{\beta}\right): \beta<\lambda\right)$ é uma partida do jogo $\mathrm{G}_{1}^{\lambda}\left(\mathcal{O}_{\mathcal{B}}, \mathcal{O}_{\mathcal{B}}\right)$ jogada em $\Phi$ e, como $\Phi$ é estratégia vencedora para o jogador II nesse jogo, inferimos que $\left\{U_{\beta}: \beta<\lambda\right\} \in \mathcal{O}_{\mathcal{B}}$. Além disso, pela suposição feita sobre $\Phi$, para qualquer $\gamma<\lambda$ temos $\left\{U_{\beta}: \gamma<\beta<\lambda\right\} \in \mathcal{O}_{\mathcal{B}}$. Afirmamos que $\left\{f_{\beta}: \beta<\lambda\right\} \in \Omega_{\underline{0}}$. De fato, para $B \in \mathcal{B}$ e $\varepsilon>0$ quaisquer, existe $\gamma<\lambda$ tal que $\frac{1}{n_{\gamma}+1}<\varepsilon$. Como $\left\{U_{\beta}: \gamma<\beta<\lambda\right\}$ é uma $\mathcal{B}$-cobertura para $X$, segue que $B \subseteq U_{\beta}=f_{\beta}^{-1}\left[I_{n_{\beta}}\right]$, para algum $\gamma<\beta<\lambda$. Enfim, como $n_{\gamma} \leq n_{\beta}$, temos $f_{\beta} \in\langle B, \varepsilon\rangle[\underline{0}]$ e, assim, $\left\{f_{\beta}: \beta<\lambda\right\} \in \Omega_{\underline{0}}$.

Observação 2.3.14. Para $\alpha=\omega$, as observações sobre ordinais aditivamente indecomponíveis não são necessárias, pois podemos usar o Lema 2.1.1. Primeiramente, afirmamos que não há perda de generalidade em supor que $X \notin \mathcal{U}_{n}\left(A_{n}\right)$ para todo $n$, onde $A_{n}$ é um lance qualquer do jogador I na $n$-ésima rodada de uma partida do jogo $\mathrm{G}_{1}\left(\Omega_{\underline{0}}, \Omega_{\underline{0}}\right)$. De fato, se $\Phi$ é a estratégia vencedora do jogador II em $\mathrm{G}_{1}\left(\mathcal{O}_{\mathcal{B}}, \mathcal{O}_{\mathcal{B}}\right)$, podemos definir a estratégia $\eta$ para o jogador II em $\mathrm{G}_{1}\left(\Omega_{\underline{0}}, \Omega_{\underline{0}}\right)$ fazendo $\eta\left(\left(A_{0}, \ldots, A_{n}\right)\right)=f$, onde $f \in A_{n}$ é tal que $f^{-1}\left[I_{n}\right]=X$ caso tal $f$ exista, ou $f \in A_{n}$ satisfaz $f^{-1}\left[I_{n}\right]=\Phi\left(\left(\mathcal{U}_{j}\left(A_{j}\right): j \leq n\right.\right.$ e $\left.\left.X \notin \mathcal{U}_{j}\left(A_{j}\right)\right)\right)$ caso contrário - intuitivamente, $\eta$ utiliza $\Phi$ somente quando os lances do jogador I não induzem coberturas triviais. Claramente, tal estratégia reduz nossa análise aos casos em que $P=\left(\left(A_{n}, f_{n}\right): n \in \omega\right)$ é uma partida jogada em $\eta$ na qual $X \in \mathcal{U}_{n}\left(A_{n}\right)$ ocorre somente finitas vezes, mas, em tais situações, $P^{\prime}=\left(\left(A_{n}, f_{n}\right): n \in \omega\right.$ e $\left.X \notin \mathcal{U}_{n}\left(A_{n}\right)\right)$ corresponde a uma partida jogada em $\eta$, o que prova a afirmação. Enfim, tendo $X \notin \mathcal{U}_{n}\left(A_{n}\right)$ para todo $n \in \omega$ e chamando $f_{n}^{-1}\left[I_{n}\right]=U_{n}$, temos $\left(\left(\mathcal{U}_{n}\left(A_{n}\right), U_{n}\right): n \in \omega\right)$ uma partida em $\mathrm{G}_{1}\left(\mathcal{O}_{\mathcal{B}}, \mathcal{O}_{\mathcal{B}}\right)$ jogada em $\Phi$ e, por conseguinte, 
vencida pelo jogador II. Isso implica que $\left\{U_{n}: n \in \omega\right\}$ é uma $\mathcal{B}$-cobertura não trivial para $X$ e daí, usando o Lema 2.1.1 como fizemos no Teorema 2.3.6, segue que $\left\{f_{n}: n \in \omega\right\} \in \Omega_{\underline{0}}$.

Voltemos para a demonstração do Teorema.

Reciprocamente, seja $\psi$ uma estratégia vencedora para o jogador II no jogo $\mathrm{G}_{1}^{\alpha}\left(\Omega_{\underline{0}}, \Omega_{\underline{0}}\right)$. Para $\mathcal{U} \in \mathcal{O}_{\mathcal{B}}$ não trivial, o Lema 2.3.1 nos diz que $\mathcal{A}(\mathcal{U}) \in \Omega_{\underline{0}}$. Com isso, vamos definir uma estratégia $\rho$ para o jogador II no jogo $\mathrm{G}_{1}^{\alpha}\left(\mathcal{O}_{\mathcal{B}}, \mathcal{O}_{\mathcal{B}}\right)$ da seguinte maneira:

- $\operatorname{se}\left(\mathcal{U}_{\gamma}: \gamma \leq \beta\right)$ é uma sequência de comprimento $\beta+1<\alpha$ de $\mathcal{B}$-coberturas não triviais, fazemos $\rho\left(\left(\mathcal{U}_{\gamma}: \gamma \leq \beta\right)\right)=U \in \mathcal{U}_{\beta}$, onde $U$ é tal que $\psi\left(\left(A\left(\mathcal{U}_{\gamma}\right): \gamma \leq \beta\right)\right) \uparrow X \backslash U \equiv 1$;

- para toda cobertura $\mathcal{U} \in \mathcal{O}_{\mathcal{B}}$ que contém $X$, fazemos $\sigma\left(\left(\mathcal{U}_{\gamma}: \gamma \leq \beta\right)^{\frown}(\mathcal{U})\right)=X$, para qualquer sequência $\left(\mathcal{U}_{\gamma}: \gamma \leq \beta\right) \in \mathcal{O}_{\mathcal{B}}^{<\alpha}$

- nos demais casos, $\sigma\left(\left(\mathcal{U}_{\gamma}: \gamma \leq \beta\right)\right) \in \mathcal{U}_{\beta}$ é um elemento arbitrário de $\mathcal{U}_{\beta}$.

O fato de $\psi$ ser uma estratégia para o jogador II no jogo $\mathrm{G}_{1}^{\alpha}\left(\Omega_{\underline{0}}, \Omega_{0}\right)$ e o Lema 2.3 .1 garantem que $\rho$ é uma estratégia para o jogador II no jogo $\mathrm{G}_{1}^{\alpha}\left(\mathcal{O}_{\mathcal{B}}, \mathcal{O}_{\mathcal{B}}\right)$. Veremos que $\rho$ é vencedora.

Seja $\left(\left(\mathcal{U}_{\beta}, U_{\beta}\right): \beta<\alpha\right)$ uma partida jogada em $\rho$. Pela forma como definimos $\rho$, podemos supor que os lances $\mathcal{U}_{\beta}$ do jogador I são $\mathcal{B}$-coberturas não triviais, donde segue que $\mathcal{A}\left(\mathcal{U}_{\beta}\right) \in \Omega_{\underline{0}}$ para todo $\beta<\alpha$. Ainda pela definição de $\rho$, para cada $\beta<\alpha$ existe $f_{\beta} \in \mathcal{A}\left(\mathcal{U}_{\beta}\right)$ satisfazendo $f_{\beta} \uparrow X \backslash U_{\beta} \equiv 1$, tal que $\left(\left(\mathcal{A}\left(\mathcal{U}_{\beta}\right), f_{\beta}\right): \beta<\alpha\right)$ é uma partida jogada em $\psi$. Como $\psi$ é uma estratégia vencedora, temos $\left\{f_{\beta}: \beta<\alpha\right\} \in \Omega_{\underline{0}}$, e isso acarreta $\left\{U_{\beta}: \beta<\alpha\right\} \in \mathcal{O}_{\mathcal{B}}$.

As implicações do teorema acima para $C_{p}$-teoria e $C_{k}$-teoria seguem nos corolários abaixo:

Corolário 2.3.15 (Scheepers, [40], Teorema 23). Seja $\alpha \geq \omega$ um ordinal enumerável.

(a) II $\uparrow \mathrm{G}_{1}^{\alpha}\left(\Omega_{\underline{0}}, \Omega_{\underline{0}}\right)$ em $C_{p}(X)$ se, e somente se, II $\uparrow \mathrm{G}_{1}^{\alpha}(\Omega, \Omega)$.

(b) II $\uparrow \mathrm{G}_{f i n}^{\alpha}\left(\Omega_{\underline{0}}, \Omega_{\underline{0}}\right)$ em $C_{p}(X)$ se, e somente se, $I I \uparrow \mathrm{G}_{f i n}^{\alpha}(\Omega, \Omega)$.

Corolário 2.3.16. Seja $\alpha \geq \omega$ um ordinal enumerável.

(a) II $\uparrow \mathrm{G}_{1}^{\alpha}\left(\Omega_{\underline{0}}, \Omega_{\underline{0}}\right)$ em $C_{k}(X)$ se, e somente se, $I I \uparrow \mathrm{G}_{1}^{\alpha}(\mathcal{K}, \mathcal{K})$.

(b) II $\uparrow \mathrm{G}_{f i n}^{\alpha}\left(\Omega_{\underline{0}}, \Omega_{\underline{0}}\right)$ em $C_{k}(X)$ se, e somente se, II $\uparrow \mathrm{G}_{\text {fin }}^{\alpha}(\mathcal{K}, \mathcal{K})$. 
No que concerne ao jogador I, algumas caracterizações análogas são conhecidas para $C_{p}(X)$. Em [39], Scheepers apresenta os seguintes resultados:

Teorema 2.3.17 (Scheepers, [39], Teoremas 11 e 13). Seja X um espaço de Tychonoff.

(a) As seguintes afirmações são equivalentes:

(1) $C_{p}(X)$ satisfaz $\mathrm{S}_{1}\left(\Omega_{\underline{0}}, \Omega_{\underline{0}}\right)$;

(2) $I \ngtr \mathrm{G}_{1}\left(\Omega_{\underline{0}}, \Omega_{\underline{0}}\right)$ em $C_{p}(X)$;

(3) $I \ngtr \mathrm{G}_{1}(\Omega, \Omega)$ em $X$;

(4) X satisfaz $\mathrm{S}_{1}(\Omega, \Omega)$.

(b) As seguintes afirmações são equivalentes:

(1) $C_{p}(X)$ satisfaz $\mathrm{S}_{\text {fin }}\left(\Omega_{\underline{0}}, \Omega_{\underline{0}}\right)$;

(2) $I \ngtr \mathrm{G}_{f i n}\left(\Omega_{\underline{0}}, \Omega_{\underline{0}}\right)$ em $C_{p}(X)$;

(3) $I \ngtr \mathrm{G}_{\text {fin }}(\Omega, \Omega)$ em $X$;

(4) $X$ satisfaz $\mathrm{S}_{f i n}(\Omega, \Omega)$.

Em vista do que já provamos, os resultados acima suscitam a seguinte questão:

Questão 2.3.1. Vale algo análogo ao Teorema 2.3.17 para $\mathcal{K}$ e $C_{k}(X)$ ou, mais geralmente, para $\mathcal{O}_{\mathcal{B}}$ e $C_{\mathcal{B}}(X)$ ?

Ainda não conseguimos resultados na direção " $S_{1} \Rightarrow I \not \forall G_{1}$ ", contudo, como no Teorema 2.3.13, relacionamos as estratégias do jogador $\mathrm{I}$ em $\mathrm{G}_{1}\left(\mathcal{O}_{\mathcal{B}}, \mathcal{O}_{\mathcal{B}}\right)$ e $\mathrm{G}_{1}\left(\Omega_{\underline{0}}, \Omega_{\underline{0}}\right)$ (bem como para a variação $G_{f i n}$ ), no seguinte:

\section{Teorema 2.3.18.}

(a) $I \uparrow \mathrm{G}_{1}\left(\mathcal{O}_{\mathcal{B}}, \mathcal{O}_{\mathcal{B}}\right)$ se, e somente se, $I \uparrow \mathrm{G}_{1}\left(\Omega_{\underline{0}}, \Omega_{\underline{0}}\right)$ em $C_{\mathcal{B}}(X)$.

(b) $I \uparrow \mathrm{G}_{f i n}\left(\mathcal{O}_{\mathcal{B}}, \mathcal{O}_{\mathcal{B}}\right)$ se, e somente se, $I \uparrow \mathrm{G}_{f i n}\left(\Omega_{\underline{0}}, \Omega_{\underline{0}}\right)$ em $C_{\mathcal{B}}(X)$. 
Demonstração. Novamente, como $(a)$ e $(b)$ são análogos, provaremos somente o item $(a)$.

Sejam $\tau$ a topologia de $X$ e $\rho$ uma estratégia vencedora para o jogador $\mathrm{I}$ em $\mathrm{G}_{1}\left(\mathcal{O}_{\mathcal{B}}, \mathcal{O}_{\mathcal{B}}\right)$. Para uma $\mathcal{B}$-cobertura $\mathcal{U}$ não trivial, o Lema 2.3.1 implica que $\mathcal{A}(\mathcal{U}) \in \Omega_{\underline{0}}$. Como $\rho$ é vencedora para o jogador I em $\mathrm{G}_{1}\left(\mathcal{O}_{\mathcal{B}}, \mathcal{O}_{\mathcal{B}}\right), \rho$ não "escolhe" coberturas triviais como lances para o jogador I. Vamos obter uma estratégia $\mu$ para o jogador I em $\mathrm{G}_{1}\left(\Omega_{\underline{0}}, \Omega_{\underline{0}}\right)$.

Defina $\mu(\emptyset)=\mathcal{A}(\rho(\emptyset))$ e note que para qualquer $f \in \mathcal{A}(\rho(\emptyset))$ escolhida pelo jogador II deve existir $U_{f} \in \rho(\emptyset)$ tal que $f \uparrow X \backslash U_{f} \equiv 1$. Para $n \geq 1$, sejam uma sequência $\left(f_{0}, \ldots, f_{n-1}\right) \in C_{\mathcal{B}}(X)^{n}$ e $\left(U_{0}, \ldots, U_{n-1}\right) \in \tau^{n}$ tais que

- $U_{m} \in \rho\left(\left(U_{0}, \ldots, U_{m-1}\right)\right)$ para todo $m \leq n-1 \mathrm{e}$

- $f_{m} \uparrow X \backslash U_{m} \equiv 1$ para todo $m \leq n-1$, i.e., $f_{m} \in \mathcal{A}\left(\rho\left(\left(U_{0}, \ldots, U_{m-1}\right)\right)\right)$ para todo $m \leq n-1$;

nessas condições, fazemos $\mu\left(\left(f_{0}, \ldots, f_{n-1}\right)\right)=\mathcal{A}\left(\rho\left(\left(U_{0}, \ldots, U_{n-1}\right)\right)\right)$. Para as sequências $s$ que não satisfazem as condições acima, $\mu(s)$ será irrelevante para o jogo, pois tais sequências não representam respostas legais para o jogador II. Veremos que o jogador I sempre vence em $\mathrm{G}_{1}\left(\Omega_{\underline{0}}, \Omega_{\underline{0}}\right)$ com a estratégia $\mu$.

Seja $\left(\left(A_{n}, f_{n}\right): n \in \omega\right)$ uma partida jogada em $\mu$. Temos assim $f_{0} \in A_{0}=\mathcal{A}(\rho(\emptyset))$ e $U_{0} \in$ $\rho(\emptyset)$ tais que $f_{0}\left\lceil X \backslash U_{0} \equiv 1, f_{1} \in A_{1}=\mathcal{A}\left(\rho\left(\left(U_{0}\right)\right)\right)\right.$ e $U_{1} \in \rho\left(\left(U_{0}\right)\right)$ tais que $f_{1} \uparrow X \backslash U_{1} \equiv 1$ e, mais geralmente para $n>1, f_{n} \in A_{n}=\mathcal{A}\left(\rho\left(\left(U_{0}, \ldots, U_{n-1}\right)\right)\right)$ e $U_{n} \in \rho\left(\left(U_{0}, \ldots, U_{n-1}\right)\right)$ tais que $f_{n}\left\lceil X \backslash U_{n} \equiv 1\right.$, donde segue que $\left(\left(\rho\left(\left(U_{m}: m<n\right)\right), U_{n}\right): n \in \omega\right)$ é uma partida do jogo $\mathrm{G}_{1}\left(\mathcal{O}_{\mathcal{B}}, \mathcal{O}_{\mathcal{B}}\right)$ jogada em $\rho$. Logo, $\left\{U_{n}: n \in \omega\right\} \notin \mathcal{O}_{\mathcal{B}}$, o que nos dá $B \in \mathcal{B}$ tal que $B \not \subset U_{n}$ para todo $n$, e disso decorre que $\langle B, 1\rangle[\underline{0}] \cap\left\{f_{n}: n \in \omega\right\}=\emptyset$, mostrando que $\left\{f_{n}: n \in \omega\right\} \notin \Omega_{\underline{0}}$.

Reciprocamente, considere $\psi$ uma estratégia vencedora para o jogador I em $\mathrm{G}_{1}\left(\Omega_{\underline{0}}, \Omega_{\underline{0}}\right)$ em $C_{\mathcal{B}}(X)$. Para cada $A \in \Omega_{\underline{0}}$ e $n \in \omega$, vamos considerar a $\mathcal{B}$-cobertura $\mathcal{U}_{n}(A)$, como no Lema 2.3.1. Chamaremos uma sequência finita $P=\left(\left(A_{n}, f_{n}\right): n<m\right)$ de início de partida jogada em $\psi$ (de comprimento $m$ ) se $f_{n} \in A_{n}=\psi\left(\left(f_{j}: j<n\right)\right)$ para todo $n<m$. Para dois inícios de partida $P_{0}$ e $P_{1}$ de comprimentos $m_{0}$ e $m_{1}$, respectivamente, diremos que $P_{1}$ estende $P_{0}$ se $m_{0} \leq m_{1}$ e $P_{1} \uparrow m_{0} \equiv P_{0}$. Para obtermos uma estratégia vencedora $\Phi$ para o jogador I em $\mathrm{G}_{1}\left(\mathcal{O}_{\mathcal{B}}, \mathcal{O}_{\mathcal{B}}\right)$, provamos primeiramente a seguinte: 
Afirmação 2.3.19. Seja $m \in \omega$ qualquer. Se $P^{\prime}=\left(\left(A_{n}, f_{n}\right): n<m\right)$ é um início de partida jogada em $\psi$, então para algum $p \geq m$ existe um início de partida $P=\left(\left(A_{n}, f_{n}\right): n<p+1\right)$ que estende $P^{\prime}$ tal que $X \notin \mathcal{U}_{p}\left(A_{p}\right)$.

Demonstração da Afirmação. Ora, se não for este o caso, então para todo $k \geq m$ o jogador II escolhe $g_{k} \in A_{k}$ tal que $g_{k}^{-1}\left[I_{k}\right]=X$, onde $A_{k}$ é o $k$-ésimo lance do jogador I. Assim, $\left\{g_{n}: n \in \omega\right\} \in \Omega_{\underline{0}}$, pois, para $\langle B, \varepsilon\rangle[\underline{0}]$ uma vizinhança de $\underline{0}$, basta tomar $n \geq m$ satisfazendo $\frac{1}{n+1}<\varepsilon$ e notar que $g_{n} \in\langle B, \varepsilon\rangle[\underline{0}]$, pois $g_{n}^{-1}\left[I_{n}\right]=X$.

Pela afirmação, existe $P_{0}=\left(\left(A_{0, n}, f_{0, n}\right): n<p_{0}+1\right)$ um início de partida jogada em $\psi$ que estende $P_{0}^{\prime}=\emptyset$ com $p_{0} \geq 0$ tal que $X \notin \mathcal{U}_{p_{0}}\left(A_{0, p_{0}}\right)$. Defina $\Phi(\emptyset)=\mathcal{U}_{p_{0}}\left(A_{0, p_{0}}\right)$. Se $V_{0} \in \Phi(\emptyset)$ é a resposta do jogador II em $\mathrm{G}_{1}\left(\mathcal{O}_{\mathcal{B}}, \mathcal{O}_{\mathcal{B}}\right)$ em $X$, então existe $g_{0} \in A_{0, p_{0}}$ tal que $g_{0}^{-1}\left[I_{p_{0}}\right]=V_{0} \neq X$. Novamente, pela afirmação existe um início de partida $P_{1}=\left(\left(A_{1, n}, f_{1, n}\right)\right.$ : $\left.n<p_{1}+1\right)$ jogado em $\psi$ que estende o início de partida $P_{1}^{\prime}=P_{0} \uparrow p_{0} \uparrow\left(\left(A_{0, p_{0}}, g_{0}\right)\right)$ e satisfaz $X \notin \mathcal{U}_{p_{1}}\left(A_{1, p_{1}}\right)$ para algum $p_{1} \geq p_{0}+1$. Definimos $\Phi\left(\left(V_{0}\right)\right)=\mathcal{U}_{p_{1}}\left(A_{1, p_{1}}\right)$.

Procedendo indutivamente, isto define uma estratégia $\Phi$ para o jogador $\mathrm{I}$ em $\mathrm{G}_{1}\left(\mathcal{O}_{\mathcal{B}}, \mathcal{O}_{\mathcal{B}}\right)$ em $X$. Note que se $R=\left(\left(\mathcal{V}_{n}, V_{n}\right): n \in \omega\right)$ é uma partida jogada em $\Phi$, então existe uma sequência estritamente crescente $\left(p_{n}: n \in \omega\right)$ de números naturais e uma sequência $\left(P_{m}: m \in \omega\right)$ de inícios de partidas jogadas em $\psi$, onde $P_{m}=\left(\left(A_{m, n}, f_{m, n}\right): n<p_{m}+1\right)$, tais que, para todo $n \in \omega:$

1. $P_{n+1}$ estende $P_{n} \uparrow p_{n}$

2. $V_{n}=f_{n+1, p_{n}}^{-1}\left[I_{p_{n}}\right] \neq X$ (note que $A_{n, p_{n}}=A_{n+1, p_{n}}$ e $\left.\left(A_{n+1, p_{n}}, f_{n+1, p_{n}}\right) \in P_{n+1}\right)$;

3. $\mathcal{V}_{n}=\mathcal{U}_{p_{n}}\left(A_{n, p_{n}}\right)$ é não trivial.

Observe que para a partida $R$ corresponde uma partida $P$ jogada em $\psi$ : para cada $j, n \in \omega$ considere $\left(C_{j}, h_{j}\right)=\left(A_{n, j}, f_{n, j}\right)$ sempre que $j<p_{n}$ e faça $P=\left(\left(C_{j}, h_{j}\right): j \in \omega\right)$; $P$ é uma partida jogada em $\psi$ devido à condição (1). Assim, se ocorresse $\mathcal{V}=\left\{V_{n}: n \in \omega\right\} \in \mathcal{O}_{\mathcal{B}}$, então $\mathcal{V}$ seria uma $\mathcal{B}$-cobertura não trivial e, desse modo, $\left\{V_{m}: m \geq n\right\} \in \mathcal{O}_{\mathcal{B}}$ para qualquer $n \in \omega$ (cf. Lema 2.1.1). Isso implicaria em $\left\{f_{n+1, p_{n}}: n \in \omega\right\} \in \Omega_{0}$ : dada uma vizinhança $\langle B, \varepsilon\rangle[\underline{0}]$ de $\underline{0}$, tome $n \in \omega$ satisfazendo $\frac{1}{p_{n}+1}<\varepsilon$ e note que $\left\{V_{m}: m \geq n\right\}$ é uma $\mathcal{B}$ cobertura; logo, existe $m \geq n$ tal que $B \subseteq V_{m}=f_{m+1, p_{m}}^{-1}\left[I_{p_{m}}\right]$ e, por conseguinte, para 
todo $x \in B,\left|f_{m+1, p_{m}}(x)\right|<\frac{1}{p_{m}+1} \leq \frac{1}{p_{n}+1}<\varepsilon$, mostrando que $f_{m+1, p_{m}} \in\langle B, \varepsilon\rangle[\underline{0}]$. Como $\left\{f_{m+1, p_{m}}: m \in \omega\right\} \subseteq\left\{h_{n}: n \in \omega\right\}$, a partida $P$ seria vencida pelo jogador II em $\mathrm{G}_{1}\left(\Omega_{\underline{0}}, \Omega_{\underline{0}}\right)$, absurdo.

\subsubsection{O tightness de $C_{\mathcal{B}}(X)$ e a operação produto}

De acordo com Arhangel'skii em [3], dizemos que um espaço topológico $X$ é produtivamente de tightness menor do que ou igual a $\kappa$ em $x \in X$ (ou simplesmente produtivamente $\kappa$-tight em $x$ ) se para todo espaço $Y \operatorname{com} t(Y) \leq \kappa$ valer $t((x, y), X \times Y) \leq \kappa$ para todo $y \in Y$, que abreviamos por $\kappa \in S p(x, X)$. Quando tal condição for satisfeita para todo $x \in X$, diremos que $X$ é um espaço produtivamente $\kappa$-tight, que indicamos por $\kappa \in S p(X)$.

Em [3], Arhangel'skii deu uma caracterização “interna” para um espaço topológico $X$ ser produtivamente de tightness menor do que ou igual a $\kappa$, por meio da noção de famílias singulares. Uma coleção $\mathcal{P}$ de famílias de subconjuntos de $X$ é $\kappa$-singular em $x$ caso satisfaça as seguintes condições:

(a) para todo $\xi \in \mathcal{P}$ existe $A(\xi) \in \xi$ tal que $|A(\xi)| \leq \kappa \cdot{ }^{14}$

(b) $\operatorname{se} \xi \in \mathcal{P}$, então $\xi$ tem p.i.f. (se $F \in[\xi]^{<\omega}$, então $\bigcap F \neq \emptyset$ );

(c) se $V$ é uma vizinhança de $x$ então existem $\xi \in \mathcal{P}$ e $A \in \xi$ tais que $A \subseteq V$.

(d) para toda subcoleção $\mathcal{E} \subseteq \mathcal{P} \operatorname{com}|\mathcal{E}| \leq \kappa$, podemos escolher $A(\xi) \in \xi$ para cada $\xi \in \mathcal{E}$ tais que $x \notin \overline{\bigcup_{\xi \in \mathcal{E}} A(\xi)}$.

Se existir uma família $\kappa$-singular em $x \in X$, diremos que $X$ é um espaço $\kappa$-singular em $x$ e, se tal condição for válida para todo $x \in X$, diremos simplesmente que $X$ é um espaço $\kappa$-singular.

Teorema 2.3.20 (Arhangel'skii, [3], Teorema 3.5). Sejam $X$ um espaço topológico, $x \in X$ e $\kappa$ um cardinal transfinito. $\kappa \notin S p(x, X)$ se, e somente se, existe uma família $\kappa$-singular em $x$.

Demonstração. Provaremos este teorema na Seção 2.4.3.

\footnotetext{
${ }^{14}$ Note que a condição $(a)$ é claramente equivalente à exigência $(a)^{\prime}:$ para todo $\xi \in \mathcal{P}$ tem-se $|A| \leq \kappa$ para todo $A \in \xi$
} 
Nesta subseção, vamos obter condições necessárias e suficientes para $C_{\mathcal{B}}(X)$ ser produtivamente $\kappa$-tight. Em particular, mostraremos que tal condição se traduz como uma propriedade de recobrimento em $X$ com relação à bornologia $\mathcal{B}$.

Chamemos de $\tau$ a topologia de $X$ e tomemos um cardinal $\kappa \geq \aleph_{0}$. Denotamos por $X_{\kappa} \mathrm{o}$ espaço topológico $\left(X, \tau_{\kappa}\right)$, onde

$$
\tau_{\kappa}:=\left\{\bigcap \mathcal{U}: \mathcal{U} \in[\tau]^{\leq \kappa}\right\}
$$

o espaço $X_{\kappa}$ é a $\kappa$-modificação de $X$, e os elementos de $\tau_{\kappa}$ são chamados de conjuntos $G_{\kappa}$ de $X$. Em particular, para $\kappa=\aleph_{0}$, denotamos $X_{\aleph_{0}}=X_{\delta}$ e chamamos os elementos de $\tau_{\aleph_{0}}$ de conjuntos $G_{\delta}$.

Essencialmente, $\tau_{\kappa}$ é a topologia em $X$ obtida ao declararmos como abertos todas as interseções de até $\kappa$ abertos da topologia original. Além disso, como $\mathcal{B}$ é uma bornologia em $X$, então $\mathcal{B}$ é ainda uma bornologia em $X_{\kappa}$, pois $X_{\kappa}=\left(X, \tau_{\kappa}\right)$. Assim, faz sentido denotarmos por $l_{\mathcal{B}}\left(X_{\kappa}\right)$ o menor cardinal transfinito $\lambda$ tal que para toda $\mathcal{B}$-cobertura $\mathcal{U}$ de $X_{\kappa}$ (composta por $\tau_{\kappa}$-abertos) existe $\mathcal{U}^{\prime} \in[\mathcal{U}]^{\leq \lambda}$ uma $\mathcal{B}$-subcobertura para $X_{\kappa}$.

Ainda em [3], Arhangel'skii também caracterizou $S p(x, X)$ em termos do tightness de $x$ em qualquer compactificação Hausdorff de $X$. Por meio de tal resultado, Uspenskii caracterizou completamente $S p\left(C_{p}(X)\right)$, no seguinte teorema:

Teorema 2.3.21 (Uspenskii, [48], Teorema 1). Para um espaço de Tychonoff $X, \kappa \in S p\left(C_{p}(X)\right)$ se, e somente se, $l\left(X_{\kappa}\right) \leq \kappa$.

Por meio do Teorema 2.3.20, generalizamos ${ }^{15}$ o teorema acima evitando o argumento via compactificação utilizado por Uspenskii.

Teorema 2.3.22. Para um espaço de Tychonoff $X$ munido de uma bornologia $\mathcal{B}$ com base compacta, $\kappa \in S p\left(C_{\mathcal{B}}(X)\right)$ se, e somente se, $l_{\mathcal{B}}\left(X_{\kappa}\right) \leq \kappa$.

Demonstração. Seja $\mathcal{U}$ uma $\mathcal{B}$-cobertura para $X$ composta por conjuntos $G_{\kappa}$ de $X$ que não admite $\mathcal{B}$-subcobertura de cardinalidade menor do que ou igual a $\kappa$. Vamos mostrar que $\kappa \notin$

\footnotetext{
${ }^{15}$ Agradecemos ao professor Angelo Bella pelas sugestões que permitiram provar a versão geral da "ida" no Teorema 2.3.22, isto é, $\kappa \in S p\left(C_{\mathcal{B}}(X)\right) \Rightarrow l_{\mathcal{B}}\left(X_{\kappa}\right) \leq \kappa$; originalmente tínhamos apenas $\kappa \in S p\left(C_{\mathcal{B}}(X)\right) \Rightarrow$ $l_{\mathcal{B}}\left(X_{\delta}\right) \leq \kappa$.
} 
$S p\left(C_{\mathcal{B}}(X)\right)$. Como $C_{\mathcal{B}}(X)$ é homogêneo, pelo Teorema 2.3.20 é equivalente provar que $C_{\mathcal{B}}(X)$ é $\kappa$-singular em $\underline{0}$.

Para cada $B \in \mathcal{B}$, existe $U \in \mathcal{U}$ tal que $\bar{B} \subseteq U$ e $U=\bigcap \mathcal{C}(U)$, onde $\mathcal{C}(U)$ é uma coleção de abertos de $X \operatorname{com}|\mathcal{C}(U)| \leq \kappa$. Sem perda de generalidade, podemos supor que $\mathcal{C}(U)$ é fechado por interseções finitas. Como $X$ é um espaço de Tychonoff, para cada $C \in \mathcal{C}(U)$ existe uma função $f_{B, C} \in C_{\mathcal{B}}(X)$ satisfazendo $f_{B, C} \uparrow \bar{B} \equiv 0$ e $f_{B, C} \uparrow(X \backslash C) \equiv 1$. Agora, definimos os conjuntos:

- $A_{B, U, C}=\left\{f_{B, V}: V \in \mathcal{C}(U) \wedge V \subseteq C\right\}$;

- $\mathcal{A}_{B, U}=\left\{A_{B, U, C}: C \in \mathcal{C}(U)\right\} \mathrm{e}$

- $\mathscr{P}=\left\{\mathcal{A}_{B, U}: B \in \mathcal{B} \wedge U \in \mathcal{U} \wedge \bar{B} \subseteq U\right\}$.

Afirmamos que $\mathscr{P}$ é uma família $\kappa$-singular em $\underline{0}$. Verificamos a seguir as condições da definição de família $\kappa$-singular:

(a) Pela construção, cada membro de $\mathcal{A}_{B, U}$ tem cardinalidade $\leq \kappa$.

(b) Se $\bar{B} \subseteq U \in \mathcal{U}$ e $C_{0}, \ldots, C_{n} \in \mathcal{C}(U)$, então $C=\bigcap_{i \leq n} C_{i} \in \mathcal{C}(U)$ e, pelo modo como tomamos as coleções, temos $f_{B, C} \in \bigcap_{i \leq n} A_{B, U, C_{i}}$, donde segue que $\mathcal{A}_{B, U}$ tem p.i.f.

(c) $\operatorname{Se}\langle B, \varepsilon\rangle[\underline{0}]$ é uma vizinhança de $\underline{0}$, então existe $U \in \mathcal{U}$ com $\bar{B} \subseteq U$, o que nos permite inferir que $A_{B, U, C} \subseteq\langle B, \varepsilon\rangle[\underline{0}]$ para todo $C \in \mathcal{C}(U)$.

(d) Finalmente, seja $\left\{\mathcal{A}_{B_{\alpha}, U_{\alpha}}: \alpha<\kappa\right\} \subseteq \mathscr{P}$. Pela suposição feita acerca de $\mathcal{U}$, segue que $\left\{U_{\alpha}: \alpha<\kappa\right\}$ não é uma $\mathcal{B}$-cobertura para $X_{\kappa}$, e então existe $B \in \mathcal{B}$ satisfazendo $B \not \subset U_{\alpha}$ para todo $\alpha<\kappa$. Como $U_{\alpha}=\bigcap \mathcal{C}\left(U_{\alpha}\right)$, existe $C_{\alpha} \in \mathcal{C}\left(U_{\alpha}\right)$ tal que $B \backslash C_{\alpha} \neq \emptyset \mathrm{e}$, pelo modo como as funções $f_{B, C}$ foram tomadas, decorre que cada elemento de $A_{B_{\alpha}, U_{\alpha}, C_{\alpha}}$ assume valor 1 em algum ponto de $B$. Logo, a vizinhança $\left\langle B, \frac{1}{2}\right\rangle[\underline{0}]$ atesta que $\underline{0} \notin \overline{\bigcup\left\{A_{B_{\alpha}, U_{\alpha}, C_{\alpha}}: \alpha<\kappa\right\}}$. Portanto, $\mathscr{P}$ é $\kappa$-singular em $\underline{0}$.

Reciprocamente, assuma $l_{\mathcal{B}}\left(X_{\kappa}\right) \leq \kappa$. Provaremos que $C_{\mathcal{B}}(X)$ não é $\kappa$-singular em $\underline{0}$. Para tanto, seja $\mathcal{P}=\left\{\mathcal{A}_{\alpha}\right\}_{\alpha \in I}$ uma coleção de subconjuntos de $X$ satisfazendo as condições $(a)^{\prime},(b)$ e $(c)$ da definição de família $\kappa$-singular. Vamos mostrar que a condição $(d)$ não é satisfeita. 
Para cada $\alpha \in I, A \in \mathcal{A}_{\alpha}$ e $n \in \omega$, considere o conjunto $\mathcal{U}_{A, n}=\left\{f^{-1}\left[I_{n}\right]: f \in A\right\}$, e faça $\mathfrak{U}_{n}=\left\{\bigcap \mathcal{U}_{A, n}: \alpha \in I\right.$ e $\left.A \in \mathcal{A}_{\alpha}\right\}$. Afirmamos que $\mathfrak{U}_{n}$ é uma $\mathcal{B}$-cobertura para $X$ composta por conjuntos $G_{\kappa}$ de $X$. De fato, dado $B \in \mathcal{B}$, temos $\left\langle B, \frac{1}{n+1}\right\rangle[\underline{0}]$ uma vizinhança de $\underline{0}$ e, pela condição $(c)$, segue que existem $\alpha \in I$ e $A \in \mathcal{A}_{\alpha}\left(|A| \leq \kappa\right.$ pela condição $\left.(a)^{\prime}\right)$ com $A \subseteq\left\langle B, \frac{1}{n+1}\right\rangle[\underline{0}], \operatorname{logo} \mathcal{U}_{A, n}$ tem cardinalidade no máximo $\kappa$ e $B \subseteq \bigcap \mathcal{U}_{A, n}$.

Pela hipótese, cada $\mathfrak{U}_{n}$ tem uma $\mathcal{B}$-subcobertura $\mathfrak{U}_{n}^{\prime}=\left\{U_{\lambda, n}: \lambda<\kappa\right\}$. Note que para cada $\lambda<\kappa$ e $n \in \omega$ podemos escolher $\alpha_{\lambda, n} \in I$ e $C_{\lambda, n} \in \mathcal{A}_{\alpha_{\lambda, n}} \operatorname{com} U_{\lambda, n}=\bigcap \mathcal{U}_{C_{\lambda, n}, n}$. Afirmamos que $\left\{\mathcal{A}_{\alpha_{\lambda, n}}: \lambda<\kappa, n \in \omega\right\}$ atesta a não validade de $(d)$. De fato, para cada $\lambda<\kappa$ e $n \in \omega$ escolha $A_{\lambda, n} \in \mathcal{A}_{\alpha_{\lambda, n}}$. Considere $\langle B, \varepsilon\rangle[\underline{0}]$ uma vizinhança de $\underline{0}$ e fixe $n \in \omega \operatorname{com} \frac{1}{n+1}<\varepsilon$. Por $\mathfrak{U}_{n}^{\prime}$ ser uma $\mathcal{B}$-subcobertura, existe $U_{\lambda, n} \operatorname{com} B \subseteq U_{\lambda, n}$. Logo, $C_{\lambda, n} \subseteq\langle B, \varepsilon\rangle[\underline{0}]$, o que acarreta $A_{\lambda, n} \cap\langle B, \varepsilon\rangle[\underline{0}] \neq \emptyset$, pois $A_{\lambda, n} \cap C_{\lambda, n} \neq \emptyset$ pela condição $(b)$ da p.i.f. Isto encerra a prova.

Do Teorema 2.3.21 de Uspenskii e do teorema acima para $\mathcal{B}=\mathcal{F}$, obtemos uma demonstração alternativa para

$$
l_{\mathcal{F}}\left(X_{\kappa}\right) \leq \kappa \Leftrightarrow l\left(X_{\kappa}\right) \leq \kappa
$$

Além disso, para $\kappa=\aleph_{0}$, temos:

Corolário 2.3.23. Sejam $X$ um espaço de Tychonoff e $\mathcal{B}$ uma bornologia com base compacta em $X . \aleph_{0} \in S p\left(C_{\mathcal{B}}(X)\right)$ se, e somente se, $l_{\mathcal{B}}\left(X_{\delta}\right)=\aleph_{0}$.

Em particular, pelo Corolário 2.4.8 (cf. Subseção 2.4.3), segue que $\chi\left(C_{\mathcal{B}}(X)\right) \in S p\left(C_{\mathcal{B}}(X)\right)$. 


\subsection{Apêndice do Capítulo}

\subsubsection{Diagramas}

(1) Funções Cardinais

O diagrama abaixo resume as relações entre as funções cardinais que estudamos nesse capítulo. Abaixo, consideramos $X$ um espaço de Tychonoff munido de uma bornologia $\mathcal{B}$ com base compacta. As notações $\alpha \rightarrow \beta$ e $\alpha-\beta$ indicam $\beta \leq \alpha$ e $\alpha=\beta$, respectivamente.

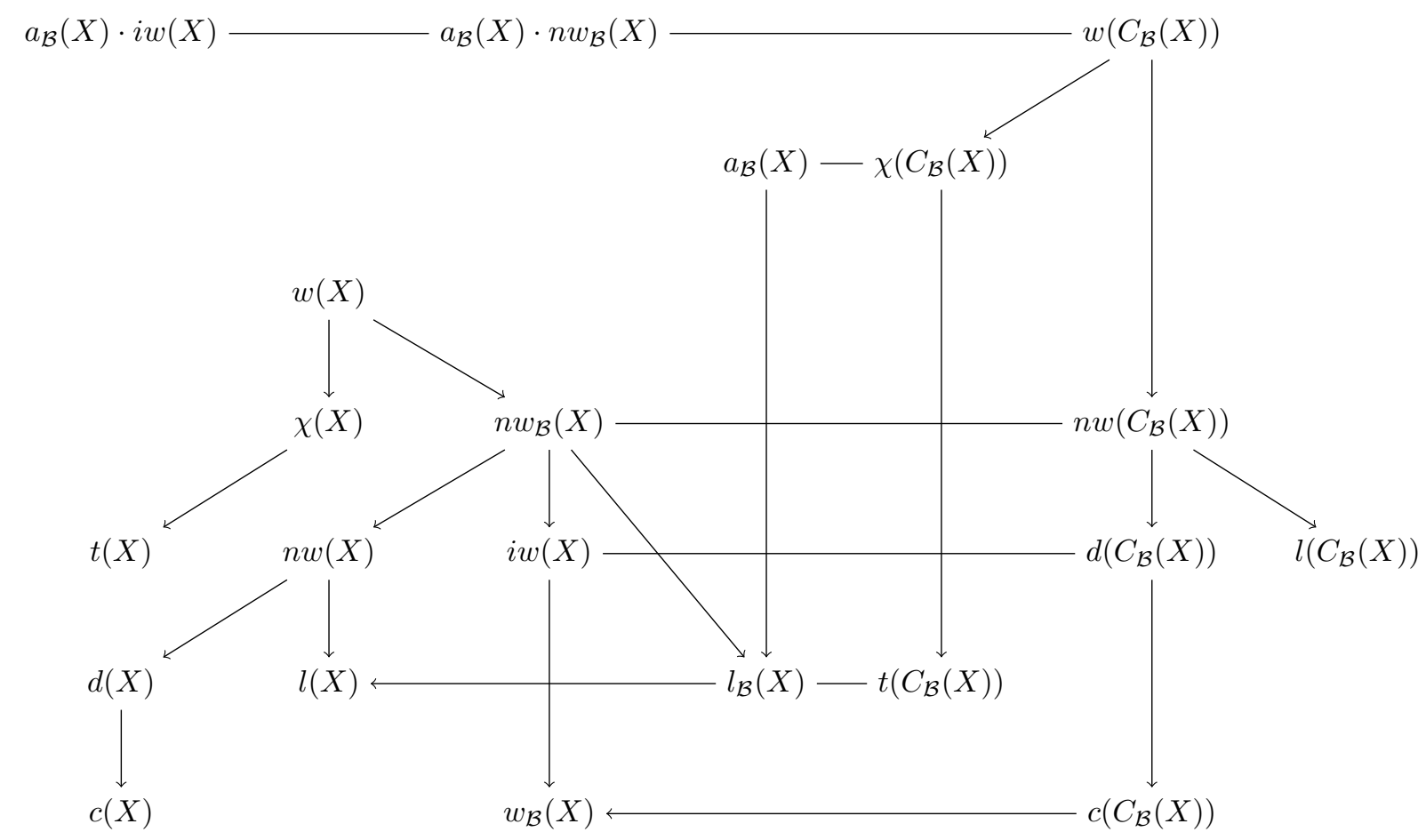




\section{(2) Variações em tightness}

No diagrama seguinte, a notação $A \stackrel{n}{\rightarrow} B$ significa que “ $A$ implica $B$ conforme provamos na Proposição (Lema, Teorema, Observação etc.) de número $n$ ' ${ }^{16}$, para $X$ espaço de Tychonoff e $\mathcal{B}$ bornologia com base compacta em $X$.

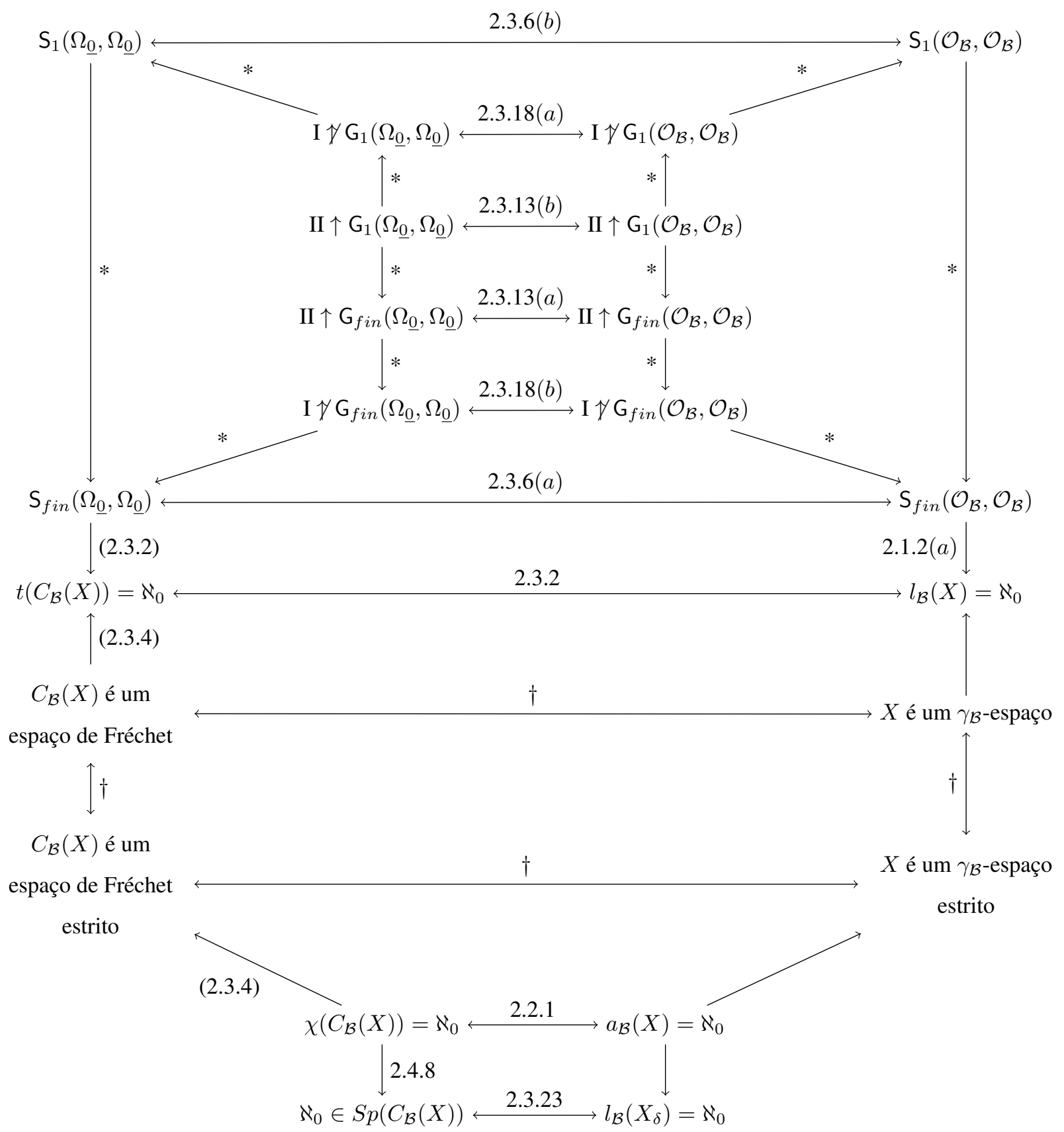

\footnotetext{
${ }^{16}$ Os símbolos $* \mathrm{e} \dagger$ correspondem à cadeia de implicações 1.4 .3 na Seção 1.4 e ao Teorema 2.3.5, respectivamente.
} 


\subsubsection{Convergência uniforme forte - o caso métrico}

Em [6], Beer e Levi definem uma topologia em $Y^{X}$ por meio de uma bornologia em $X$, para $X$ e $Y$ métricos, que generaliza (para esta classe de espaços) a topologia $\mathcal{T}_{\mathcal{B}}$ estudada ao longo deste capítulo. Esse foi o primeiro material no qual encontramos a abordagem por bornologias utilizada neste trabalho.

Os autores consideram como entourages básicas sobre $Y^{X}$ os conjuntos da forma

$$
[B, \varepsilon]^{s}:=\left\{(f, g) \in Y^{X} \times Y^{X}: \exists \delta>0\left(\forall x \in B^{\delta}(\rho(f(x), g(x))<\varepsilon)\right)\right\}
$$

com $B \in \mathcal{B}$ e $\varepsilon>0$, onde $\mathcal{B}$ é uma bornologia sobre $X$ com base fechada. A topologia gerada a partir dessa uniformidade é chamada de topologia da convergência uniforme forte em $\mathcal{B}$, e denotada por $\mathcal{T}_{\mathcal{B}}^{s}$. O nome dado a tal topologia se deve ao fato de que ela "preserva" um certo tipo de continuidade forte.

Para $(X, d)$ e $(Y, \rho)$ espaços métricos, dizemos que uma função $f: X \rightarrow Y$ é fortemente uniformemente contínua em $B \subseteq X$ (strongly uniformly continuous) se para todo $\varepsilon>0$ existe $\delta>0$ tal que $\rho(f(x), f(y))<\varepsilon$ sempre que $d(x, y)<\delta$ e $\{x, y\} \subseteq B^{\delta}$. Quando $f$ é fortemente uniformemente contínua em $B$, para todo elemento $B$ de uma família $\mathcal{B}$, dizemos que $f$ é fortemente uniformemente contínua em $\mathcal{B}$. Denotamos por $C_{\mathcal{B}}^{s}(X, Y)$ o conjunto de todas as funções de $X$ em $Y$ fortemente uniformemente contínuas em $\mathcal{B}$.

Considerando então a topologia $\mathcal{T}_{\mathcal{B}}$ da convergência uniforme em $\mathcal{B}$ - exatamente a mesma que estudamos -, prova-se a seguinte proposição:

Proposição 2.4.1 (Beer e Levi, [6], Proposições 6.3 e 6.5). Sejam $(X, d),(Y, \rho)$ espaços métricos e $\mathcal{B}$ uma bornologia de base fechada em X. Então

(a) $C_{\mathcal{B}}^{s}(X, Y)$ é fechado em $\left(Y^{X}, \mathcal{T}_{\mathcal{B}}^{s}\right)$.

(b) $\mathcal{T}_{\mathcal{B}}^{s}=\mathcal{T}_{\mathcal{B}}$ em $C_{\mathcal{B}}^{s}(X, Y)$;

Corolário 2.4.2 (Beer e Levi, [6], Teorema 6.7). Sejam $(X, d),(Y, \rho)$ espaços métricos, $\mathcal{B}$ bornologia com base fechada sobre $X$ e $\left(f_{l}\right)_{l \in L}$ uma net em $C_{\mathcal{B}}^{s}(X, Y)$. Se $f_{l} \rightarrow \mathcal{T}_{\mathcal{B}} f \in Y^{X}$, então são equivalentes: 
(a) $f \in C_{\mathcal{B}}^{s}(X, Y)$;

(b) $f_{l} \rightarrow \mathcal{T}_{\mathcal{B}}^{s} f$.

Demonstração. Como $C_{\mathcal{B}}^{s}(X, Y)$ é $\mathcal{T}_{\mathcal{B}}^{s}$-fechado, $(b) \Rightarrow(a)$ vale mesmo sem a suposição da $\mathcal{T}_{\mathcal{B}}$ -convergência de $\left(f_{l}\right)$. Supondo então $f \in C_{\mathcal{B}}^{s}(X, Y)$, inferimos pela hipótese do enunciado que $f_{l} \rightarrow_{\mathcal{T}_{\mathcal{B}}} f$, com $\left\{f_{l}: l \in L\right\} \cup\{f\} \subseteq C_{\mathcal{B}}^{s}(X, Y)$, donde a $\mathcal{T}_{\mathcal{B}}^{s}$-convergência da net segue por termos $\mathcal{T}_{\mathcal{B}}^{s}=\mathcal{T}_{\mathcal{B}}$ em $C_{\mathcal{B}}^{s}(X, Y)$.

O resultado que motivou o estudo do artigo [6] foi o seguinte corolário, no qual os autores obtém condições necessárias e suficientes para que o limite de funções contínuas seja contínuo (cf. Seção 1.2).

Corolário 2.4.3 (Beer e Levi, [6], Corolário 6.8). Sejam $(X, d),(Y, \rho)$ espaços métricos e $\left(f_{l}\right)_{l \in L}$ uma net em $C(X, Y)$. Se $f_{l} \rightarrow f \in Y^{X}$ pontualmente, então são equivalentes:

(a) $f \in C(X, Y)$;

(b) $f_{\lambda} \rightarrow{ }_{\mathcal{T}_{\mathcal{F}}} f$.

Demonstração. Basta aplicar o corolário anterior, lembrando que para $\mathcal{B}=\mathcal{F}, \mathcal{T}_{\mathcal{F}}$ é a topologia da convergência pontual e, neste caso, vale que $f_{\lambda} \rightarrow \mathcal{T}_{\mathcal{F}} f$ se, e somente se, $f_{\lambda}(x) \rightarrow f(x)$, para todo $x \in X$ (além de notar que $C(X, Y)=C_{\mathcal{F}}^{s}(X, Y)$ ).

No entanto, um resultado análogo e mais geral já havia sido apresentado por Bouleau [8], em 1969, válido para espaços da forma $Y^{X}$, com $Y$ uniforme e $X$ qualquer. Neste caso, a topologia $\mathcal{T}_{v}$ definida por Bouleau em $Y^{X}$, chamada de topologia da $V$-convergência, é induzida por uma uniformidade que tem como base todos os conjuntos da forma

$$
\langle F, \mathcal{V}\rangle_{v}:=\left\{(f, g) \in Y^{X} \times Y^{X}: \exists V \in \tau(F \subseteq V \wedge \forall x \in V((f(x), g(x)) \in \mathcal{V}))\right\},
$$

para todo $F \in[X]^{<\omega}$ e $\mathcal{V} \in \mathfrak{U}$, onde $\tau$ é a topologia de $X$ e $\mathfrak{U}$ é a uniformidade em $Y$. É fácil ver que quando $X$ e $Y$ são métricos, as definições de $\mathcal{T}_{v}$ e $\mathcal{T}_{\mathcal{F}}^{s}$ coincidem, isto é, a topologia da convergência uniforme forte na bornologia dos conjuntos finitos é a topologia da $V$ - convergência de Bouleau. Aliás, vale uma versão análoga da Proposição 2.4.1, cuja demonstração 
consiste numa adaptação dos argumentos métricos para a linguagem de uniformidades, como o que fizemos no Lema 1.3 .6 .

Proposição 2.4.4 (Bouleau, [8], Proposição 3). Sejam X espaço topológico e Y espaço uniforme. Então $C(X, Y)$ é fechado em $\left(Y^{X}, \mathcal{T}_{v}\right)$ e, além disso, $\mathcal{T}_{v}$ coincide com a topologia da convergência pontual em $C(X, Y)$.

De modo geral, quando a bornologia $\mathcal{B}$ tem base compacta, temos $C_{\mathcal{B}}(X, Y)=C_{\mathcal{B}}^{s}(X, Y)$ - o que segue do fato de que, se $f$ é contínua num compacto $K$ de $X$, então $f$ é fortemente uniformemente contínua em $K$. Assim, para espaços métricos $X$ e $Y$, a topologia $\mathcal{T}_{\mathcal{B}}^{s}$ generaliza em $C(X, Y)$ a topologia $\mathcal{T}_{\mathcal{B}}$. Em particular, valem versões análogas de muitos dos teoremas provados neste capítulo para $\left(C(X), \mathcal{T}_{\mathcal{B}}^{s}\right)$, exigindo-se apenas que $\mathcal{B}$ tenha base fechada.

De fato, como todo espaço métrico é normal, a exigência de compacidade para os elementos de uma base da bornologia $\mathcal{B}$ para a obtenção de funções contínuas (por meio do Lema de Urysohn "Fraco") torna-se desnecessária, pois em espaços normais vale o Lema de Urysohn - frisamos que, neste caso, o conceito de $\mathcal{B}$-coberturas é substituído pelo de $\mathcal{B}_{s}$-coberturas (Caserta et al. [9, 10]): $\mathcal{U}$ é uma $\mathcal{B}_{s}$ - cobertura se para todo $B \in \mathcal{B}$ existem $\delta>0$ e $U \in \mathcal{U}$ tais que $B^{\delta} \subseteq U$.

Note ainda que a necessidade de que a bornologia $\mathcal{B}$ tenha base compacta quando $X$ é apenas de Tychonoff (e não necessariamente métrico), reduz as tentativas "naturais" de generalização da topologia da convergência uniforme forte para a topologia da convergência uniforme. Por exemplo, se $(X, \tau)$ é de Tychonoff e $(Y, \mathfrak{U})$ é uniforme, podemos considerar a uniformidade em $Y^{X}$ cujas entourages básicas são da forma

$$
\langle B, \mathcal{U}\rangle^{s}:=\left\{(f, g) \in Y^{X} \times Y^{X}: \exists V \in \tau(B \subseteq V \wedge \forall x(x \in V \Rightarrow(f(x), g(x)) \in \mathcal{U}))\right\}
$$

para $B \in \mathcal{B}$ e $\mathcal{U} \in \mathfrak{U}$. Se $\mathcal{B}$ tem base compacta, usando a compacidade mostra-se facilmente que a uniformidade gerada pelos conjuntos da forma $\langle B, \mathcal{U}\rangle^{s}$ coincide com a uniformidade gerada pelos conjuntos da forma $\langle B, \mathcal{U}\rangle$ quando nos restringimos a $C(X, Y)-$ para $\langle B, \mathcal{U}\rangle^{s}$, basta tomar $\mathcal{V}$ uma entourage simétrica tal que $\mathcal{V} \circ \mathcal{V} \circ \mathcal{V} \subseteq \mathcal{U}$ e notar que $\langle\bar{B}, \mathcal{V}\rangle \subseteq\langle B, \mathcal{U}\rangle^{s}$. Mesmo supondo adicionalmente que $X$ também esteja munido de uma uniformidade, digamos $\mathfrak{V}$, a 
uniformidade em $Y^{X}$ gerada pelas entourages da forma

$$
\langle B, \mathcal{U}\rangle_{s}:=\left\{(f, g) \in Y^{X} \times Y^{X}: \exists \mathcal{V} \in \mathfrak{V}(x \in \mathcal{V}[B] \Rightarrow(f(x), g(x)) \in \mathcal{U})\right\}
$$

para $B \in \mathcal{B}$ e $\mathcal{U} \in \mathfrak{U}$, também coincide em $C(X, Y)$ com a uniformidade gerada pelas entourages $\langle B, \mathcal{U}\rangle$, o que se deve essencialmente ao Lema 1.3.6. Desse modo, fica justificada a escolha da topologia $\mathcal{T}_{\mathcal{B}}$ como forma de generalizar a topologia definida em [6]. 


\subsubsection{Demonstração do Teorema 2.3.20 (de Arhangel'skii)}

Apresentamos nesta subseção a demonstração do Teorema 2.3.20, tanto por sua importância para os resultados obtidos neste trabalho quanto pela dificuldade para encontrar sua demonstração original (em inglês). A argumentação - adaptada de [3] - requer alguns comentários preliminares, o que faremos a seguir.

Proposição 2.4.5. Seja $\left\{\left(X_{\xi}, \tau_{\xi}\right)\right\}_{\xi \in \mathcal{P}}$ uma família de espaços topológicos dois a dois disjuntos e considere $X=\sum_{\xi \in \mathcal{P}} X_{\xi}$. Então $t(X) \leq \sup \left\{t\left(X_{\xi}\right): \xi \in \mathcal{P}\right\}$.

Demonstração. Suponha $t\left(X_{\xi}\right) \leq \kappa$ para todo $\xi \in \mathcal{P}$, e tome $x \in X$ e $A \subseteq X$ tais que $x \in \bar{A}$. Como os $X_{\xi}$ 's são dois a dois disjuntos, existe um único $\xi \in \mathcal{P}$ com $x \in X_{\xi}$ e, por conseguinte, $x \in \overline{A \cap X_{\xi}} \subseteq \bar{A}$. Logo, existe $B \subseteq A \cap X_{\xi} \subseteq A$ com $|B| \leq \kappa$ tal que $x \in \bar{B}$, o que nos dá $t(X) \leq \kappa$.

Para espaços topoógicos $(Y, \tau)$ e $(Z, \sigma)$, dizemos que uma aplicação sobrejetora $\varphi: Y \rightarrow Z$ é uma função quociente se $\sigma=\left\{V \subseteq Z: \varphi^{-1}[V] \in \tau\right\}$.

Lema 2.4.6 (Arhangel'skii e Ponomarev, apud Engelking [15]). Seja X um espaço topológico.

(a) $t(X) \leq \kappa$ se, e somente se, $\bar{C}=\bigcup_{M \in[C] \leq \kappa} \bar{M}$, para todo $C \subseteq X$.

(b) $t(X)$ é o menor cardinal $\kappa$ tal que para todo $C \subseteq X$ não fechado existe $C_{0} \in[C]^{\leq \kappa}$ satisfazendo $\overline{C_{0}} \backslash C \neq \emptyset$.

(c) Se $\varphi: Y \rightarrow Z$ é uma função quociente, então $t(Z) \leq t(Y)$.

Demonstração.

(a). Decorre diretamente da definição de tightness.

(b). Suponha $t(X)=\kappa$. Se $C$ não é fechado, então existe $x \in \bar{C} \backslash C$ e, da hipótese sobre $t(X)$, segue que existe $C_{0} \in[C]^{\leq \kappa} \operatorname{com} x \in \overline{C_{0}}$, o que acarreta $\overline{C_{0}} \backslash C \neq \emptyset$. Para provar a recíproca, usaremos o item $(a)$. Considere $C \subseteq X$ qualquer; se $C$ for fechado, então $\bar{C}=$ $\bigcup_{M \in[C] \leq \kappa} \bar{M}$ trivialmente; se $C$ não for fechado, suponha por absurdo que $\bar{C} \neq \bigcup_{M \in[C] \leq \kappa} \bar{M}$, o que equivale a afirmar que $\bigcup_{M \in[C] \leq \kappa} \bar{M}:=G$ não é fechado, pois $G \subseteq \bar{C} \subseteq \bar{G}$. Logo, pela hipótese existe $C_{0} \in[G]^{\leq \kappa}$ tal que $\overline{C_{0}} \backslash G \neq \emptyset$. Para cada $c \in C_{0}$, existe $M_{c} \in[C] \leq \kappa$ tal que 
$c \in \overline{M_{c}}$, donde segue que $M=\bigcup_{c \in C_{0}} M_{c} \in[C]^{\leq \kappa}$ e $a \in \bar{M}$ para todo $a \in C_{0}$, o que acarreta $C_{0} \subseteq \bar{M}$. Assim, $\overline{C_{0}} \subseteq G$, absurdo. Portanto, $t(X) \leq \kappa$.

(c). Suponha $t(Y) \leq \kappa$ e tome $C \subseteq Z$ não fechado. Como $\varphi$ é quociente, segue que $\varphi^{-1}[C]$ não é fechado em $Y$, e o item $(b)$ nos diz que existe $F \in\left[\varphi^{-1}[C]\right]^{\leqslant \kappa}$ tal que $\bar{F} \backslash \varphi^{-1}[C] \neq \emptyset$. Assim, $\varphi[F] \in[C]^{\leq \kappa}$ e $\overline{\varphi[F]} \backslash C \neq \emptyset$, e novamente pelo item (b) concluímos que $t(Z) \leq$ $t(Y)$.

Note que o tightness de $X$ em $x$ é "limitado" pela cardinalidade de qualquer vizinhança de $x$. De fato, se $V$ é uma vizinhança de $x$ e $t(x, X)>|V|$, segue que existe $M \subseteq X$ com $|M|>|V|$ tal que $x \in \bar{M}$ e $x \notin \bar{L}$, para todo $L \in[M] \leq|V|$. Em particular, $x \notin \overline{M \cap V}$, mas isto não pode ocorrer pois, se $A$ é um aberto que contém $x$, então $x \in A \cap V$ e, por conseguinte, $M \cap(V \cap A)=(M \cap V) \cap A \neq \emptyset$, i.e., $x \in \overline{M \cap V}$.

Lema 2.4.7 (Arhangel'skii, [3]). Sejam $X$ um espaço topológico, $x \in X$ e $\mathcal{P}$ uma família $\kappa$-singular em $x$.

(a) Podemos supor que cada $\xi \in \mathcal{P}$ é fechado por interseções finitas.

(b) Se $t(x, X) \leq \kappa$, então não há perda de generalidade em supor que $\bigcap \xi=\emptyset$, para todo $\xi \in \mathcal{P}$.

Demonstração. (a). Basta tomar $\mathcal{P}^{\prime}=\left\{\xi^{\prime}: \xi \in \mathcal{P}\right\}$, onde $\xi^{\prime}:=\left\{\bigcap F: F \in[\xi]^{<\omega}\right\}$, que satisfaz as condições de família $\kappa$-singular em $x$.

(b). Neste caso, é suficiente observar que $\mathcal{P}^{*}=\{\xi \in \mathcal{P}: \cap \xi=\emptyset\}$ também é uma família $\kappa$ singular em $x$. Suponha que não seja este o caso, e chame de $\mathcal{P}^{\prime}=\{\xi \in \mathcal{P}: \bigcap \xi \neq \emptyset\}=\mathcal{P} \backslash \mathcal{P}^{*}$.

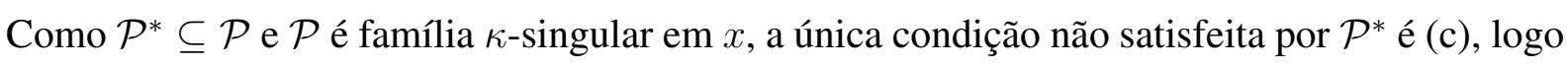
deve existir uma vizinhança $U_{0}$ de $x$ tal que $E \not \subset U_{0}$ para todo $E \in \bigcup \mathcal{P}^{*}$. Por outro lado, como $\mathcal{P}$ satisfaz (C) por hipótese, para toda vizinhança $V$ de $x$ existe $\xi(V) \in \mathcal{P}^{\prime}$ tal que $C(V) \subseteq V \cap U_{0}$ para algum $C(V) \in \xi(V)$. Pela forma como tomamos $\mathcal{P}^{\prime}$, temos

$$
V \supseteq C(V) \supseteq \bigcap \xi(V) \neq \emptyset
$$

assim, chmanado $F=\bigcup\left\{\bigcap \xi: \xi \in \mathcal{P}^{\prime}\right\}$, temos em verdade $V \cap F \neq \emptyset$ para toda vizinhança de $x$, o que nos dá $x \in \bar{F}$. Agora, usamos a hipótese de que $t(x, X) \leq \kappa$ para obter $F^{\prime} \in[F]^{\leq \kappa}$ 
tal que $x \in \overline{F^{\prime}}$. Para cada $y \in F^{\prime}$, fixe $\xi(y) \in \mathcal{P}^{\prime} \operatorname{com} y \in \bigcap \xi(y)$, chame $\mathcal{E}=\{\xi(y): y \in$ $\left.F^{\prime}\right\} \subseteq \mathcal{P}^{\prime} \subseteq \mathcal{P}$ e note que $|\mathcal{E}| \leq \kappa$. Como $\mathcal{P}$ é $\kappa$-singular em $x$, para cada $y \in F^{\prime}$ podemos escolher $A(y) \in \xi(y)$ tal que $x \notin \overline{\bigcup_{y \in F^{\prime}} A(y)}$; mas se $y \in F^{\prime}$, então $y \in \bigcap \xi(y) \subseteq A(y)$, donde temos $F^{\prime} \subseteq \bigcup_{y \in F^{\prime}} A(y)$ e, por conseguinte, $\overline{F^{\prime}} \subseteq \overline{\bigcup_{y \in F^{\prime}} A(y)}$, o que implica $x \notin \overline{F^{\prime}}$, uma contradição.

Demonstração (do Teorema 2.3.20). Suponha que $\kappa \notin S p(x, X)$. Isto significa que existem um espaço $Y \operatorname{com} t(Y) \leq \kappa$ e $y \in Y$ tais que $t((x, y), X \times Y)>\kappa$. Pela definição de tightness, existe $M \subseteq X \times Y$ com $|M|>\kappa$ tal que $(x, y) \in \bar{M}$ e $(x, y) \notin \bar{L}$ para todo $L \in[M]^{\leq \kappa}$. Com tais hipóteses, para cada vizinhança $V_{x}$ de $x$ vamos obter uma família $\xi\left(V_{x}\right)$ de subconjuntos de $V_{x}$ de modo que a família $\mathcal{P}=\left\{\xi\left(V_{x}\right): V_{x}\right.$ é vizinhança de $\left.x\right\}$ será $\kappa$-singular em $x$.

Fixemos $V_{x}$ uma vizinhança de $x$. Chame $M^{\prime}=M \cap \pi_{X}^{-1}\left[V_{x}\right] \subseteq X \times Y$ e $L=\pi_{Y}\left[M^{\prime}\right] \subseteq Y$, onde $\pi_{X}$ e $\pi_{Y}$ são as projeções canônicas de $X \times Y$ sobre $X$ e $Y$, respectivamente. Note que $y \in \bar{L}:$ de fato, se $W$ é uma vizinhança de $y$ em $Y$, então $V_{x} \times W$ é uma vizinhança de $(x, y)$ em $X \times Y$ e, como $(x, y) \in \bar{M}$, inferimos que existe $(u, v) \in M \cap\left(V_{x} \times W\right)$, o que nos dá $(u, v) \in M \cap \pi_{X}^{-1}\left[V_{x}\right] \operatorname{com} v \in W$, i.e., $v \in L \cap W$.

Como $t(Y) \leq \kappa$ e $y \in \bar{L}$, segue que existe $R \in[L]^{\leq \kappa} \operatorname{com} y \in \bar{R}$ e, como $R \subseteq L=\pi_{Y}\left[M^{\prime}\right]$ e $\pi_{Y}$ é uma sobrejeção, podemos ${ }^{17}$ obter $Q \subseteq M^{\prime}$ de modo que $\pi_{Y} \uparrow Q: Q \rightarrow R$ seja uma bijeção. Agora, definimos

$$
\xi\left(V_{x}\right):=\left\{\pi_{X}\left[\pi_{Y}^{-1}[W] \cap Q\right]: W \text { é vizinhança de } y\right\}:=\vartheta(Q) .
$$

Para uma vizinhança $W$ qualquer de $y$, como $|Q| \leq \kappa$ e $Q \subseteq M^{\prime}=M \cap \pi_{X}^{-1}\left[V_{x}\right]$, temos respectivamente $\left|\pi_{X}\left[\pi_{Y}^{-1}[W] \cap Q\right]\right| \leq \kappa$ e $\pi_{X}\left[\pi_{Y}^{-1}[W] \cap Q\right] \subseteq V_{x}$. Mostremos que $\xi\left(V_{x}\right)$ tem p.i.f: se $W_{0}, \ldots, W_{n}$ são vizinhanças de $y$, então $W=\bigcap_{i \leq n} W_{i}$ é vizinhança de $y$ e, como $y \in \bar{R}$ e $R=\pi_{Y}[Q]$, existe $(q, r) \in Q$ tal que $r \in R \cap W \subseteq W_{i}$ para todo $i \leq n$, o que nos dá

$$
q \in \bigcap_{i \leq n} \pi_{X}\left[\pi_{Y}^{-1}\left[W_{i}\right] \cap Q\right] .
$$

Procedendo da maneira acima para cada vizinhança $V$ de $x$, obtemos $\mathcal{P}=\{\xi(V): V$ é vizinhança de $x\}$. Pelo que mostramos acima, resta apenas provarmos que $\mathcal{P}$ satisfaz a condição

\footnotetext{
${ }^{17}$ Como $\pi_{Y}$ é sobre, para cada $r \in R$ existe $q_{r} \in M^{\prime}$ tal que $\pi_{Y}\left(q_{r}\right)=r$; assim, basta fazermos $Q=\left\{q_{r}: r \in\right.$ $R\}$.
} 
(d) de família $\kappa$-singular. Seja $\mathcal{E} \subseteq \mathcal{P}$ com $|\mathcal{E}| \leq \kappa$ e, para cada $\xi \in \mathcal{E}$, tome $Q_{\xi} \subseteq M$ tal que $\xi=\vartheta\left(Q_{\xi}\right)$. Como $\left|Q_{\xi}\right| \leq \kappa$ para todo $\xi$, temos $N=\bigcup_{\xi \in \mathcal{E}} Q_{\xi} \in[M]^{\leq \kappa}$ e, da hipótese sobre $M$, decorre que $(x, y) \notin \bar{N}$. Obtemos assim $V \subseteq X$ e $W \subseteq Y$ vizinhanças de $x$ e $y$, respectivamente, tais que

$$
(V \times W) \cap N=\pi_{X}^{-1}[V] \cap \pi_{Y}^{-1}[W] \cap N=\emptyset .
$$

Pela definição dos membros de $\mathcal{P}, \pi_{X}\left[\pi_{Y}^{-1}[W] \cap Q_{\xi}\right] \in \xi$ para todo $\xi \in \mathcal{E}$. Agora, note que

$$
\begin{gathered}
V \cap \bigcup_{\xi \in \mathcal{E}} \pi_{X}\left[\pi_{Y}^{-1}[W] \cap Q_{\xi}\right]=V \cap \pi_{X}\left[\bigcup_{\xi \in \mathcal{E}} \pi_{Y}^{-1}[W] \cap Q_{\xi}\right]=V \cap \pi_{X}\left[\pi_{Y}^{-1}[W] \cap\left(\bigcup_{\xi \in \mathcal{E}} Q_{\xi}\right)\right]= \\
=V \cap \pi_{X}\left[\pi_{Y}^{-1}[W] \cap N\right]=\emptyset .
\end{gathered}
$$

Portanto, $\mathcal{P}$ é uma família $\kappa$-singular em $x$.

Reciprocamente, suponha que exista uma família $\mathcal{P} \kappa$-singular em $x$. Vamos obter um espaço $Z \operatorname{com} t(Z) \leq \kappa$ e $z \in Z$ tais que $t((x, z), X \times Z)>\kappa$, i.e., mostraremos que $\kappa \notin S p(x, X)$. Primeiramente, note que se $t(x, X)>\kappa$, basta tomarmos $Z$ um conjunto unitário. Assim, podemos assumir que $t(x, X) \leq \kappa$. Logo, pelo Lema 2.4.7, não há perda de generalidade em supor que todo $\xi \in \mathcal{P}$ é fechado por interseções finitas e satisfaz $\bigcap \xi=\emptyset$.

Consideremos agora a seguinte lista de "objetos" (essencialmente como na ilustração abaixo):

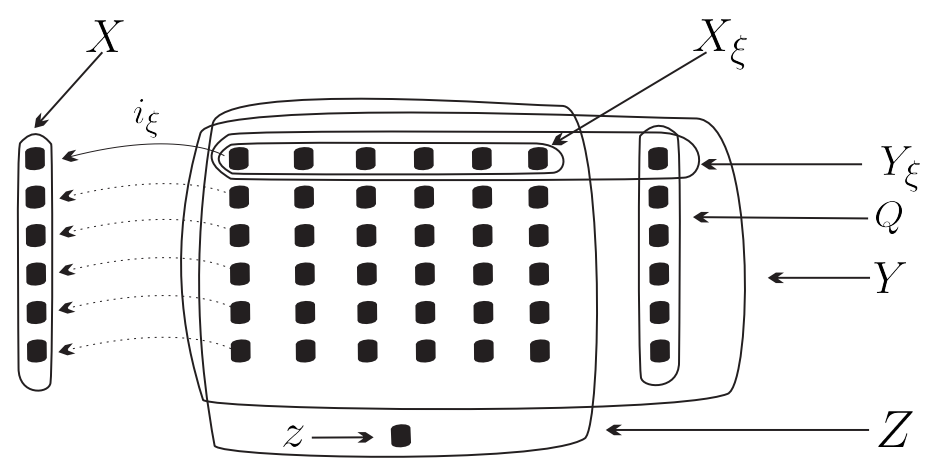

- uma família $\left\{X_{\xi}: \xi \in \mathcal{P}\right\}$ de conjuntos dois a dois disjuntos, tais que $\left|X_{\xi}\right|=|X|$, e chame $T=\bigcup_{\xi \in \mathcal{P}} X_{\xi}$

- uma família $\left\{i_{\xi}: \xi \in \mathcal{P}\right\}$ de funções $i_{\xi}: X_{\xi} \rightarrow X$ bijetoras; 
- uma família $Q=\left\{y_{\xi}: \xi \in \mathcal{P}\right\}$ de conjuntos dois a dois distintos tais que para todo $y \in Q$ tenha-se $y \notin T$, chame $Y_{\xi}=X_{\xi} \cup\left\{y_{\xi}\right\}$ para cada $\xi \in \mathcal{P}$ e $Y=\bigcup_{\xi \in \mathcal{P}} Y_{\xi}$;

- um elemento $z \notin Y$, e chame $Z=T \cup\{z\}$.

Defina agora $\varphi: Y \rightarrow Z$ por

$$
\varphi(y):=\left\{\begin{array}{l}
y \quad \text {, se } y \in T \\
z, \text { se } y \in Q
\end{array}\right.
$$

que é claramente uma função sobrejetora.

Vamos definir topologias $\tau_{\xi}$ em $Y_{\xi}, \tau$ em $Y$ e $\sigma$ em $Z$ tais que $t(Z) \leq \kappa$.

$\tau_{\xi}$. Declaramos os pontos de $X_{\xi}$ isolados, e fazemos de $B_{\xi}:=\left\{\left\{y_{\xi}\right\} \cup i_{\xi}^{-1}[U]: U \in \xi\right\}$ uma base local em $y_{\S}^{18}$ Em particular, note que $y_{\xi}$ é o único ponto não isolado de $Y_{\xi}$ nesta topologia, pois todo aberto que contém $y_{\xi}$ deve conter a imagem inversa por $i_{\xi}$ de algum $U \in \xi$. Como $\mathcal{P}$ é $\kappa$-singular, existe $U \in \xi$ tal que $|U| \leq \kappa$ e, assim, $\left\{y_{\xi}\right\} \cup$ $i_{\xi}^{-1}[U]$ é uma vizinhança de $y_{\xi}$ de cardinalidade $\leq \kappa$, donde segue que $t\left(y_{\xi}, Y_{\xi}\right) \leq \kappa$ e, consequentemente, $t\left(Y_{\xi}\right) \leq \kappa$. Além disso, pela suposição de que $\bigcap \xi=\emptyset$, temos $\bigcap B_{\xi}=\left\{y_{\xi}\right\}$ e, como todos os demais pontos são isolados, isto implica que $\left(Y_{\xi}, \tau_{\xi}\right)$ é $T_{1}$ e, por conseguinte, normal.

$\tau$. Declaramos $\tau=\left\{A \subseteq Y: \forall \xi \in \mathcal{P}\left(A \cap Y_{\xi} \in \tau_{\xi}\right)\right\}$, isto é, $(Y, \tau):=\sum_{\xi \in \mathcal{P}}\left(Y_{\xi}, \tau_{\xi}\right)$. Da definição de $\tau_{\xi}$ e da Proposição 2.4.5 temos $t(Y) \leq \kappa$. Além disso, por construção, nenhum ponto de $Q=\left\{y_{\xi}: \xi \in \mathcal{P}\right\}$ é isolado em $Y$, e $T$ é um subespaço discreto de $Y$; em particular, como cada $Y_{\xi}$ é normal, segue que $Y$ também é normal.

$\sigma$. Faremos de $\varphi$ uma aplicação quociente, declarando $\sigma=\left\{V \subseteq Z: \varphi^{-1}[V] \in \tau\right\}$. Pelo item $(c)$ do Lema 2.4.6, já temos $t(Z) \leq t(Y) \leq \kappa$ (em particular, $z$ é o único ponto não isolado de $Z$ e, como todos os seus pontos são fechados, temos $Z$ um espaço $T_{1}$ e, por conseguinte, $Z$ é normal).

\footnotetext{
${ }^{18}$ Sem a suposição de que $\xi$ é fechado por interseções finitas, teríamos $B_{\xi}$ uma sub-base local em $y_{\xi}$.
} 
Finalmente, provaremos que $t((x, z), X \times Z)>\kappa$. Afirmamos que

$$
M=\left\{\left(u, \varphi\left(i_{\xi}^{-1}(u)\right)\right): u \in X e \xi \in \mathcal{P}\right\} \subseteq X \times Z
$$

atesta a desigualdade procurada. Primeiramente, devemos mostrar que $(x, z) \in \bar{M}$.

Sejam $V \subseteq X$ e $W \subseteq Z$ vizinhanças de $x$ e $z$, respectivamente. Como $\mathcal{P}$ é $\kappa$-singular, existem $\xi^{\prime} \in \mathcal{P}$ e $A^{\prime} \in \xi^{\prime} \operatorname{com} A^{\prime} \subseteq V$. Pela definição de $\sigma, W$ é aberto em $Z$ se, e somente se, $\varphi^{-1}[W]$ é aberto em $Y$ e, como $Q=\varphi^{-1}[\{z\}] \subseteq \varphi^{-1}[W]$, temos em particular $y_{\xi^{\prime}} \in \varphi^{-1}[W]$. Pela forma como definimos as topologias de $Y$ e $Y_{\xi^{\prime}}$, existe $A^{\prime \prime} \in \xi^{\prime}$ tal que $\left\{y_{\xi^{\prime}}\right\} \cup i_{\xi^{\prime}}^{-1}\left[A^{\prime \prime}\right] \subseteq$ $\varphi^{-1}[W]$, o que implica $\varphi\left[i_{\xi^{\prime}}^{-1}\left[A^{\prime \prime}\right]\right] \subseteq W$. Agora usamos a hipótese de que cada $\xi$ é fechado por interseções finitas para garantir $A=A^{\prime} \cap A^{\prime \prime} \in \xi^{\prime}$, que é não vazio pela p.i.f. Assim, se $u \in A$, temos $u \in A^{\prime}$ e $u \in A^{\prime \prime}$, que acarretam respectivamente $u \in V$ e $\varphi\left(i_{\xi^{\prime}}^{-1}(u)\right) \in W$, i.e., $M \cap(V \times W) \neq \emptyset$.

Agora, para $L \in[M]^{\leq \kappa}$, mostraremos que $(x, z) \notin \bar{L}$. Defina

$$
\mathcal{E}=\left\{\xi \in \mathcal{P}: L \cap\left(X \times \varphi\left[X_{\xi}\right]\right) \neq \emptyset\right\}
$$

Como os $X_{\xi}$ 's são dois a dois disjuntos (e $\varphi$ fixa tais $X_{\xi}$ 's), temos necessariamente $|\mathcal{E}| \leq$ $|L| \leq \kappa$. Como $\mathcal{P}$ é $\kappa$-singular em $x$, para cada $\xi \in \mathcal{E}$ podemos escolher $A(\xi) \in \xi$ tal que $x \notin \overline{\bigcup_{\xi \in \mathcal{E}} A(\xi)}=F$. Note que $V=X \backslash F$ é um aberto que contém $x$. Definamos então

$$
W=\left(\bigcup_{\xi \in \mathcal{E}} \varphi\left[i_{\xi}^{-1}[A(\xi)]\right]\right) \cup\left(\bigcup_{\xi \in \mathcal{P} \backslash \mathcal{E}} \varphi\left[X_{\xi}\right]\right) \cup\{z\}
$$

que é um aberto de $Z$ que contém $z$. Afirmamos que $(V \times W) \cap L=\emptyset$.

Suponha que não e tome $(v, w) \in(V \times W) \cap L$. Como $(v, w) \in L \subseteq M$, temos necessariamente $(v, w)=\left(v, \varphi\left(i_{\xi_{0}}^{-1}(v)\right)\right)$, para algum $\xi_{0} \in \mathcal{P}$ e, pela forma como tomamos $i_{\xi}$, isto implica em $w \neq z$, o que garante a unicidade de $\xi_{0}$. Porém, como $(V \times W) \cap L \neq \emptyset$, temos em verdade $\xi_{0} \in \mathcal{E}$ e, portanto, $w \in \varphi\left[i_{\xi_{0}}^{-1}\left[A\left(\xi_{0}\right)\right]\right]$. Contudo, $w=\varphi\left(i_{\xi_{0}}^{-1}(v)\right)$ e $\varphi$ é injetora quando restrita a $T$, logo sua restrição a $X_{\xi_{0}}$ também é injetora. Portanto, $\varphi \circ i_{\xi_{0}}^{-1}$ é uma injeção, e disto segue que $v \in A\left(\xi_{0}\right)$, o que contradiz $v \in V$ e $A\left(\xi_{0}\right) \subseteq X \backslash V$. Isto nos mostra que $V \times W$ é uma 
vizinhança de $(x, z)$ que não intercepta $L$ e, como $L \in[M]^{\leq \kappa}$ é arbitrário, concluímos enfim que $t((x, z), X \times Z)>\kappa$.

Corolário 2.4.8. Para um espaço topológico $X, \chi(X) \in S p(X)$.

Demonstração. Chame $\kappa=\chi(X)$. Pelo Teorema 2.3.20, basta provar que para todo $x \in X$ não existe uma família $\mathcal{P} \kappa$-singular em $x$. Ora, fixando $\mathcal{P}$ uma coleção de famílias de subconjuntos de $X$ satisfazendo as condições (a), (b) e (c) de família $\kappa$-singular em $x$, mostraremos que $\mathcal{P}$ não satisfaz a condição (d). Como $\kappa=\chi(X)$, existe $\mathfrak{B}_{x}$ uma base local em $x$ de cardinalidade $\kappa$. Pela condição (c), para cada $B \in \mathfrak{B}_{x}$ existem $\xi(B) \in \mathcal{P}$ e $C(B) \in \xi(B) \operatorname{com} C(B) \subseteq B$. Logo, a família $\left\{\xi(B): B \in \mathfrak{B}_{x}\right\} \in[\mathcal{P}]^{\leq \kappa}$ é tal que $x \in \overline{\bigcup_{B \in \mathfrak{B}_{x}} A(B)}$ para qualquer escolha de elementos $A(B) \in \xi(B)$ que façamos.

Corolário 2.4.9. Todo espaço metrizável é produtivamente de tightness enumerável. 



\section{Capítulo}

\section{A Compactificação de Stone-Čech}

A compacidade é uma das propriedades topológicas mais importantes que um espaço pode possuir, não só por sua estabilidade via diversas operações, mas também por simplificar diversos contextos que poderiam ser intratáveis em sua ausência. Muitas vezes, quando um espaço não é compacto, podemos contornar parcialmente tal situação por meio de uma compactificação, que intuitivamente consiste de um espaço compacto que contém densamente o espaço original.

Neste capítulo, estudaremos algumas propriedades da compactificação de Stone-Čech, definida para espaços de Tychonoff de modo geral. Tal espaço pode ser visto como um subespaço compacto de $[0,1]^{C(X,[0,1])}$, onde $X$ é o espaço de Tychonoff - o que também justifica a presença de tal tópico neste trabalho.

\subsection{Existência da compactificação de Stone-Čech}

Para um espaço topológico $X$ de Hausdorff, diremos que uma compactificação do espaço $X$ é um par $(K, h)$, onde $K$ é um compacto Hausdorff e $h: X \rightarrow K$ é um mergulho de $X$ como um subespaço denso de $K$, isto é, $h$ é uma aplicação contínua, aberta e injetora, tal que $\overline{h[X]}=K$. Muitas vezes, o mergulho $h$ da definição é a inclusão, i.e., o compacto $K$ "realmente" contém $X$. Em vista disso, e do fato de que, a menos de homeomorfismo, $X$ é um subespaço de $K$, muitas vezes cometeremos o seguinte abuso de notação: diremos que uma 
compactificação para um espaço de Hausdorff $X$ é um compacto Hausdorff $K$ que contém $X$ satisfazendo $\bar{X}=K$.

Vejamos alguns exemplos naturais:

1. $[0,1]$ é uma compactificação de $] 0,1[$;

2. $S_{1}=\left\{(x, y) \in \mathbb{R}^{2}:|x-y|=1\right\}$ é uma compactificação de $\mathbb{R}$ (via projeção estereográfica);

3. $\omega+1$ é uma compactificação de $\omega$. Mais geralmente, $\alpha+1$ é uma compactificação de $\alpha$, para todo ordinal limite $\alpha$ ( $c f$. Exemplo3.1.19).

Suponha que $\mathcal{K}$ seja uma compactificação para o espaço $X$ com a seguinte propriedade, que chamaremos de propriedade da extensão de funções contínuas: se $K$ é compacto Hausdorff e $f: X \rightarrow K$ é contínua, então existe $F: \mathcal{K} \rightarrow K$ extensão contínua de $f$. Claramente, tal propriedade caracteriza $\mathcal{K}$, a menos de homeomorfismo. Com efeito, se $\mathcal{K}^{\prime}$ é outra compactificação de $X$ com a mesma propriedade, então existem $f_{1}: \mathcal{K}^{\prime} \rightarrow \mathcal{K}$ e $f_{2}: \mathcal{K} \rightarrow \mathcal{K}^{\prime}$ contínuas, cujas compostas restritas a $X$ coincidem com a função identidade, donde segue que $f_{1}$ e $f_{2}$ são inversas (contínuas) uma da outra, pois $X$ é denso em $\mathcal{K}$ e $\mathcal{K}^{\prime}$.

Para um espaço de Tychonoff $X$, denotamos por $\beta X$ qualquer compactificação de $X$ com a propriedade da extensão de funções contínuas. Chamamos $\beta X$ de compactificação de StoneČech de $X$, e o espaço $X^{*}:=\beta X \backslash X$ é o resíduo de $X$.

Nesta seção, provaremos que todo espaço de Tychonoff admite uma compactificação de Stone-Čech e deduziremos algumas de suas propriedades básicas. Para tanto, "mergulharemos" o espaço num compacto Hausdorff da forma $[0,1]^{I}$ para um $I$ adequado e tomaremos o seu fecho neste espaço.

Sejam $X$ um conjunto não vazio, $\left\{X_{\alpha}\right\}_{\alpha \in A}$ uma família de conjuntos não vazios e $\mathcal{F}=$ $\left\{f_{\alpha}\right\}_{\alpha \in A}$ uma família de funções, com $f_{\alpha} \in X_{\alpha}^{X}$. Chamamos de função diagonal de $X$ em $\prod_{\alpha \in A} X_{\alpha}$ a aplicação definida por

$$
\begin{aligned}
\Delta_{\alpha \in A} f_{\alpha}: X & \rightarrow \prod_{\alpha \in A} X_{\alpha} \\
x & \mapsto\left(f_{\alpha}(x)\right)_{\alpha \in A}
\end{aligned}
$$


Note que se cada $X_{\alpha}$ estiver munido de uma topologia $\tau_{\alpha}$, então $\left\{f_{\alpha}^{-1}\left[U_{\alpha}\right]: U_{\alpha} \in \tau_{\alpha}\right.$ e $\alpha \in A\}$ é sub-base para uma topologia sobre $X$, que chamamos de topologia fraca induzida pela família $\mathcal{F}$.

No caso em que $X$ e $X_{\alpha}$ são espaços topológicos, para cada $\alpha \in A$, dizemos que $\mathcal{F}$ separa pontos de $X$ se, para quaisquer $x \neq y$ em $X$, existe $\alpha \in A$ tal que $f_{\alpha}(x) \neq f_{\alpha}(y) ; \mathcal{F}$ separa pontos de fechados de $X$ se, para quaisquer $F \subseteq X$ fechado e $x \in X \backslash F$, existir $\alpha \in A$ tal que $f_{\alpha}(x) \notin \overline{f_{\alpha}[F]}$.

Lema 3.1.1. Sejam $(X, \tau)$ um espaço topológico, $\left\{\left(X_{\alpha}, \tau_{\alpha}\right)\right\}_{\alpha \in A}$ uma família de espaços topológicos e $\mathcal{F}=\left\{f_{\alpha}\right\}_{\alpha \in A}$ uma família de funções, com $f_{\alpha} \in X_{\alpha}^{X}$. A função $\Delta_{\alpha \in A} f_{\alpha}$ é um mergulho se, e somente se, $\tau$ é a topologia fraca induzida pela família $\mathcal{F}$ e $\mathcal{F}$ separa pontos de $X$.

Demonstração. Chamemos $\Delta_{\alpha \in A} f_{\alpha}=e$. Notemos que $\pi_{\alpha}$ oe $=f_{\alpha}$, onde $\pi_{\alpha}: \prod_{\alpha \in A} X_{\alpha} \rightarrow X_{\alpha}$ é a projeção canônica na $\alpha$-ésima coordenada.

Suponha que e seja um mergulho. Então $e[X]$ (com a topologia de subespaço de $\prod_{\alpha \in A} X_{\alpha}$ ) é homeomorfo a $X$. Em particular, $A$ é um aberto de $X$ se, e somente se, $e[A]$ é um aberto de $e[X]$, mas por ser aberto, inferimos que existem $V_{\alpha_{0}}, \ldots, V_{\alpha_{n}}$ abertos de $X_{\alpha_{0}}, \ldots, X_{\alpha_{n}}$, respectivamente, tais que $e[A]=\bigcap_{i \leq n} \pi_{\alpha_{i}}^{-1}\left[V_{\alpha_{i}}\right] \cap e[X]$. Logo, $A=\bigcap_{i \leq n} f_{\alpha_{i}}^{-1}\left[V_{\alpha_{i}}\right]$, o que mostra que $\tau$ é a topologia fraca induzida por $\mathcal{F}$. Além disso, como $e$ é um mergulho, para $x \neq y$ segue que $e(x) \neq e(y)$, o que pela definição de $e$ ocorre se, e somente se, existe $f_{\alpha} \in \mathcal{F}$ tal que $f_{\alpha}(x) \neq f_{\alpha}(y)$.

Reciprocamente, suponha que $\mathcal{F}$ separe pontos de $X$ e que $\tau$ seja a topologia fraca induzida por $\mathcal{F}$. Como, para cada $\alpha \in A, \pi_{\alpha} \circ e=f_{\alpha}$ é contínua, segue que $e$ é uma função contínua. Por $\mathcal{F}$ separar pontos, dados $x \neq y$ em $X$, existe $f_{\alpha} \in \mathcal{F}$ tal que $f_{\alpha}(x) \neq f_{\beta}(x)$, donde $e(x) \neq e(y)$ e, por conseguinte, $e$ é injetora. Resta mostrar que e é uma aplicação aberta. Como os abertos de $X$ são interseções finitas de conjuntos da forma $f_{\alpha}^{-1}[V]$ para $V$ aberto em $X_{\alpha}$ e $e$ é injetora, será suficiente mostrarmos que $e\left[f_{\alpha}^{-1}[V]\right]$ é aberto em $e[X]$. Note então que

$$
f_{\alpha}^{-1}[V]=\left(\pi_{\alpha} \circ e\right)^{-1}[V]=\left(\left(\pi_{\alpha}\lceil e[X]) \circ e\right)^{-1}[V]=e^{-1}\left[\left(\pi_{\alpha}\lceil e[X])^{-1}[V]\right] .\right.\right.
$$

Portanto, $e\left[f_{\alpha}^{-1}[V]\right]=\left(\pi_{\alpha}\lceil e[X])^{-1}[V]=\pi_{\alpha}^{-1}[V] \cap e[X]\right.$, como queríamos. 
Lema 3.1.2. Sejam $(X, \tau)$ um espaço topológico, $\left\{\left(X_{\alpha}, \tau_{\alpha}\right)\right\}_{\alpha \in A}$ uma família de espaços topológicos e $\mathcal{F}=\left\{f_{\alpha}\right\}_{\alpha \in A}$ uma família de funções, com $f_{\alpha} \in C\left(X, X_{\alpha}\right)$. $\mathcal{F}$ separa pontos de fechados em $X$ se, e somente se, os conjuntos da forma $f_{\alpha}^{-1}[V]$, com $\alpha \in A$ e $V \subseteq X_{\alpha}$ aberto, constituem uma base para $\tau$. Em particular, se $\mathcal{F}$ separa pontos de fechados de X, então $\tau$ é a topologia fraca induzida por $\mathcal{F}$.

Demonstração. Se os conjuntos da forma $f_{\alpha}^{-1}[V]$ constituem uma base para $\tau$, então para $x \notin F$ com $F$ fechado em $X$ existem $\beta \in A$ e $V_{\beta} \subseteq X_{\beta}$ aberto tais que $x \in f_{\beta}^{-1}\left[V_{\beta}\right] \subseteq X \backslash F$. Logo, $V_{\beta}$ é um aberto de $X_{\beta}$ tal que $f_{\beta}(x) \in V_{\beta}$ e $V_{\beta} \cap f_{\beta}[F]=\emptyset$.

Reciprocamente, supondo que $\mathcal{F}$ separe pontos de fechados, sejam $x \in X$ e $U \subseteq X$ aberto não trivial tal que $x \in U$. Temos $x \notin X \backslash U$, donde segue que existe $\beta \in A$ com $f_{\beta}(x) \notin$ $\overline{f_{\beta}[X \backslash U]}$, o que implica a existência de $V_{\beta} \subseteq X_{\beta}$ aberto tal que $f_{\beta}(x) \in V_{\beta}$ e $V_{\beta} \cap f_{\beta}[X \backslash U]=$ $\emptyset$. Notemos por fim que $x \in f_{\beta}^{-1}\left[V_{\beta}\right] \subseteq U$.

Corolário 3.1.3 (Teorema da Diagonal). Sejam $(X, \tau)$ um espaço $T_{1},\left\{\left(X_{\alpha}, \tau_{\alpha}\right)\right\}_{\alpha \in A}$ uma família de espaços topológicos e $\mathcal{F}=\left\{f_{\alpha}\right\}_{\alpha \in A}$ uma família de funções que separa pontos de fechados em $X$, tal que $f_{\alpha} \in C\left(X, X_{\alpha}\right)$. Então $\Delta_{\alpha \in A} f_{\alpha}$ é um mergulho.

Demonstração. Como o $X$ é $T_{1}, \mathcal{F}$ separar pontos de fechados implica em $\mathcal{F}$ separar pontos. Pelo lema anterior, obtemos também que a topologia $\tau$ é a topologia fraca induzida por $\mathcal{F}$. Assim o resultado segue do Lema 3.1.1.

Teorema 3.1.4 (Stone, apud Walker [49]). Todo espaço de Tychonoff possui uma compactificação de Stone- $\check{C} e c h$.

Demonstração. De fato, se $X$ é um espaço de Tychonoff, então a família $\mathcal{F}=C(X,[0,1])$ separa pontos de fechados em $X$, donde segue, pelo corolário anterior, que

$$
\Delta_{f \in \mathcal{F}} f: X \rightarrow \prod_{f \in \mathcal{F}}[0,1]
$$

é um mergulho. Agora, note que $\prod_{f \in \mathcal{F}}[0,1]$ é um compacto Hausdorff e, por conseguinte,

$$
\mathcal{K}:=\overline{\Delta_{f \in \mathcal{F}} f[X]}
$$


é um compacto Hausdorff (por ser um fechado de $\left.\prod_{f \in \mathcal{F}}[0,1]\right)$. Por construção, $\left(\mathcal{K}, \Delta_{f \in \mathcal{F}} f\right)$ é uma compactificação de $X$. Resta mostrar que $\mathcal{K}=\beta X$, isto é, que $\mathcal{K}$ tem a propriedade da extensão de funções contínuas, o que segue das duas próximas afirmações.

Afirmação 3.1.5. Dada $f: X \rightarrow[0,1]$ contínua, existe (única) $\beta f: \mathcal{K} \rightarrow[0,1]$ contínua tal que $\beta f \uparrow X=f$.

Prova da afirmação. Identificando $x=e(x)$ para todo $x$, basta definirmos $\beta f=\pi_{f} \uparrow \beta X$,

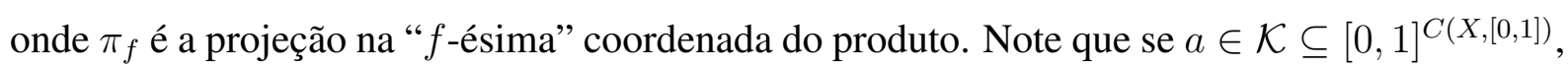
então $a=\left(y_{g}\right)_{g \in C(X,[0,1])}$ é uma função escolha, onde cada $y_{g} \in[0,1]$. Assim, $\beta f \uparrow X=f$, pois como $e(x)=(g(x))_{g \in C(X,[0,1])}$, temos

$$
\beta f(x):=\pi_{f}\left((g(x))_{g \in C(X,[0,1])}\right)=f(x) .
$$

A unicidade se deve à densidade de $X$ em $\mathcal{K}$.

Afirmação 3.1.6. Sejam $X$ um espaço de Tychonoff e $Y$ uma compactificação de $X$. São equivalentes:

(a) dado K compacto Hausdorff e $f: X \rightarrow K$ contínua, existe $\tilde{f}: Y \rightarrow K$ tal que $\tilde{f} \uparrow X=f$;

(b) dada $g: X \rightarrow[0,1]$ contínua, existe $\tilde{g}: Y \rightarrow[0,1]$ tal que $\tilde{g} \uparrow X=g$.

Em ambos os casos, as extensões são únicas.

Prova da afirmação. A unicidade das extensões decorre da densidade de $X$ em $Y$. Como $(a) \Rightarrow$ (b) é trivial, resta apenas provar que $(b) \Rightarrow(a)$. Como $K$ é compacto Hausdorff, a menos de homeomorfismo podemos supor $K \subseteq[0,1]^{I}$ para algum $I$ não vazio. Defina então $f_{i}=\pi_{i} \circ f$ para cada $i \in I$ (note que, pela identificação feita, tem-se $f(x)=\left(f_{i}(x)\right)_{i \in I}$ ).

Pela hipótese $(b)$, para cada $i \in I$ existe $\tilde{f}_{i}: Y \rightarrow[0,1]$ contínua satisfazendo $\tilde{f}_{i} \uparrow X=f_{i}$. Defina $\tilde{f}: Y \rightarrow[0,1]^{I}$ por $\tilde{f}(y)=\left(\tilde{f}_{i}(y)\right)_{i \in I}$. Temos $\tilde{f}$ contínua pois sua composta com cada uma das projeções de $[0,1]^{I}$ é contínua. Além disso, para $x \in X$ temos

$$
\tilde{f}(x)=\left(\tilde{f}_{i}(x)\right)_{i \in I}=\left(f_{i}(x)\right)_{i \in I}=f(x) .
$$


Resta mostrar que $\tilde{f}[Y] \subseteq K$. Suponha $\tilde{f}(y) \notin K$ para algum $y \in Y$. Por $K$ ser fechado, existe $A \subseteq[0,1]^{I}$ aberto com $\tilde{f}(y) \in A$ e $A \cap K=\emptyset$. Por fim, como $\tilde{f}^{-1}[A] \neq \emptyset$ e $X$ é denso em $Y$, existe $x \in X \operatorname{com} x \in \tilde{f}^{-1}[A] \cap Y \neq \emptyset$, mas então $A \cap K \neq \emptyset$, pois $\tilde{f}(x)=f(x) \in K$.

O leitor familizariado com (alguns aspectos da) Teoria das Categorias certamente notou que a compactificação de Stone-Čech define um funtor da categoria Tyc, dos espaços de Tychonoff, para a categoria CHaus, dos espaços compactos Hausdorff:

$$
\begin{aligned}
\beta: \text { Tyc } & \rightarrow \text { CHaus } \\
X & \mapsto \beta X
\end{aligned}
$$

onde a seta $f: X \rightarrow K$ é associada à extensão $\beta f: \beta X \rightarrow \beta K=K(c f$. Corolários 3.1.9 e 3.1.10 a seguir). Em particular, para todo compacto Hausdorff $K$, existe uma bijeção entre $\operatorname{hom}(\beta X, K)$ e hom $(X, K)$. Ao leitor interessado nas relações entre Teoria das Categorias e a compactificação de Stone-Čech sugerimos o Capítulo 10 do livro de Walker [49].

Recordemos que um espaço de Banach é um par $\left(E,\|\cdot\|_{E}\right)$, onde $E$ é um espaço vetorial munido de uma norma $\|\cdot\|_{E}$, no qual a métrica induzida por tal norma é completa. Se $X$ é um espaço topológico qualquer, então $C^{*}(X):=\{f \in C(X): f$ é limitada $\}$ é um subespaço vetorial de $C(X)$, e a norma $\|$.$\| dada por \|f\|:=\sup _{x \in X}|f(x)|$, para cada $f \in C^{*}(X)$, faz de $\left(C^{*}(X),\|\|.\right)$ um espaço de Banach ( $c f$. Proposição 4.13 [17]). Em particular, para qualquer espaço compacto Hausdorff $K, C(K)=C^{*}(K)$ e, por conseguinte, $C(K)$ é um espaço de Banach com a mesma norma definida acima.

Proposição 3.1.7. Para todo espaço de Tychonoff $X,(C(\beta X),\|\|$.$) é isometricamente isomorfo$ $a\left(C^{*}(X),\|\|.\right)$, i.e., existe uma bijeção $\mathbb{R}$-linear $\varphi: C(\beta X) \rightarrow C^{*}(X)$, tal que $\|\varphi(f)\|=\|f\|$ para toda $f \in C(\beta X)$. Em particular, $C(\beta \omega)$ é isometricamente isomorfo a $l^{\infty}:=\left\{f \in \mathbb{R}^{\omega}\right.$ : $\left.\sup _{n \in \omega}|f(n)|<+\infty\right\}$.

Demonstração. Basta definir $\varphi: C(\beta X) \rightarrow C^{*}(X)$ por $\varphi(f)=f \uparrow X$. Claramente $\varphi$ está bem definida, e da propriedade de extensão de funções contínuas de $\beta X$ decorre a sobrejetividade 
de $\varphi$. A linearidade de $\varphi$ é imediata. Agora, como $X \subseteq \beta X$, para $f \in C(\beta X)$ temos

$$
\|\varphi(f)\|:=\sup _{x \in X}|\varphi(f)(x)|=\sup _{x \in X}|f(x)| \leq \sup _{x \in \beta X}|f(x)|:=\|f\| .
$$

Por outro lado, como $f$ é contínua e $\beta X$ é compacto, segue que existe $x_{0} \in \beta X$ tal que $\left|f\left(x_{0}\right)\right|=\|f\| \mathrm{e}$, para $\varepsilon>0$ qualquer, existe um aberto $A \subseteq \beta X$ satisfazendo $x_{0} \in A$ e $\left|f(x)-f\left(x_{0}\right)\right|<\varepsilon$ para todo $x \in A$. Como $X$ é denso em $\beta X$, decorre que existe $x \in X \cap A$ e, por conseguinte,

$$
\|f\|=\left|f\left(x_{0}\right)\right| \leq\left|f\left(x_{0}\right)-f(x)\right|+|f(x)|<\varepsilon+|\varphi(f)(x)| \leq \varepsilon+\|\varphi(f)\| .
$$

Portanto, $\varphi$ é uma isometria e, a fortiori, é injetora.

Como todo espaço compacto Hausdorff é normal, segue que todo espaço que admite uma compactificação Hausdorff é de Tychonoff. Por tal motivo, pelo restante deste capítulo assumiremos que todos os espaços topológicos considerados são de Tychonoff - em particular, $X$ é um espaço de Tychonoff fixado. A seguir, listamos alguns corolários que seguem imediatamente do Teorema 3.1.4.

Corolário 3.1.8. Se $\mathcal{C}$ é uma compactificação de $X$ tal que toda função $f: X \rightarrow[0,1]$ admite extensão contínua $F: \mathcal{C} \rightarrow[0,1]$, então $\mathcal{C}$ é homeomorfo a $\beta X$.

Corolário 3.1.9. Se $X$ é um compacto Hausdorff, então $\beta X$ é homeomorfo a $X$

Corolário 3.1.10. Se K é uma compactificação de um espaço $Y$, então para toda função contínua $f: X \rightarrow Y$ existe uma função contínua $\beta f: \beta X \rightarrow K$ tal que $\beta f(x)=f(x)$ para todo $x \in X$. Em particular, existe $\beta f: \beta X \rightarrow \beta Y$.

Demonstração. Se $f: X \rightarrow Y$ é uma função contínua, então $\tilde{f}: X \rightarrow K$ dada por $\tilde{f}(x)=$ $f(x)$ é contínua, donde segue que existe $\beta f: \beta X \rightarrow K$.

Corolário 3.1.11. Se K é uma compactificação para $X$, então $K$ é imagem contínua de $\beta X$ por alguma função contínua que fixa os pontos de X. 
Demonstração. Como $X \subseteq K$, o corolário anterior aplicado à função inclusão $i: X \hookrightarrow K$ nos dá $\beta i: \beta X \rightarrow K$ uma extensão contínua de $i$. Agora, $\beta i[\beta X]$ é compacto em $K$ e, por conseguinte, também é fechado, o que acarreta $K=\overline{i[X]}=\overline{\beta i[X]} \subseteq \overline{\beta i[\beta X]}=\beta i[\beta X]$.

Podemos induzir uma "ordem parcial" na classe das compactificações de $X$ da seguinte maneira: se $K_{1}$ e $K_{2}$ são compactificações de $X$, dizemos que $K_{1} \leq K_{2}$ se, e somente se, existir uma sobrejeção contínua de $K_{2}$ sobre $K_{1}$ que fixe os pontos de $X$. Assim, o corolário acima mostra que $\beta X$ é a "maior" compactificação desta classe com esta ordem, o que nos dá uma demonstração alternativa para a sua unicidade (a menos de homeomorfismo).

Recordemos que um espaço topológico $X$ é localmente compacto se para todo ponto de $X$ existir um sistema fundamental de vizinhanças compactas. Quando $X$ é um espaço de Hausdorff, a condição de compacidade local se resume à existência de uma vizinhança compacta para cada ponto do espaço. Finalmente, se $X$ for um espaço de Hausdorff não compacto, então $X$ é localmente compacto se, e somente se, existe uma compactificação $K$ para $X$ tal que $|K \backslash X|=1$, a qual chamamos de compactificação de Alexandroff de $X$ (cf. Willard [50], Problema 19A).

Corolário 3.1.12. Para um espaço de Tychonoff $X, X$ é localmente compacto se, e somente se, $\beta X \backslash X$ éfechado em $\beta X$.

Demonstração. Se $X \subseteq K$ é aberto em $K$, onde $K$ é um compacto Hausdorff, então $X$ é localmente compacto. Reciprocamente, se $X$ for localmente compacto, então existe uma compactificação $K=X \cup\{P\}$ de $X$, onde $P \notin X$. Pelo corolário anterior, existe uma função contínua e sobrejetora $f: \beta X \rightarrow K$ que fixa $X$, donde segue que $\beta X \backslash X$ é fechado em $\beta X$ : como $X$ é denso em $\beta X$ e $f \nmid X: X \rightarrow X \subset K$ é um homeomorfismo, temos $f(\beta X \backslash X) \cap f(X)=\emptyset$ (cf. Lema 3.5.6, [15]), donde segue que $\beta X \backslash X=f^{-1}[\{P\}]$.

Corolário 3.1.13 (Čech, apud Walker [49]). Se $A, B \subseteq X$ são completamente separados em $X$, então $\bar{A} \cap \bar{B}=\emptyset$ em $\beta X$. Reciprocamente, se K é uma compactificação de X tal que $\bar{A} \cap \bar{B}=\emptyset$ sempre que $A, B \subseteq X$ forem completamente separados em $X$, então $K$ é homeomorfo a $\beta X$.

Demonstração. Recorde que $A, B \subseteq X$ são completamente separados em $X$ se existir uma função contínua $f: X \rightarrow[0,1]$ tal que $f(x)=0$ para todo $x \in A$ e $f(x)=1$ para todo $x \in B$. 
Assim, se $A, B \subseteq X$ são completamente separados por $f$, então existe uma extensão contínua $\beta f: \beta X \rightarrow[0,1]$ para $f$, donde segue que $\bar{A} \subseteq \beta f^{-1}[\{0\}]$ e $\bar{B} \subseteq \beta f^{-1}[\{1\}]$, o que acarreta $\bar{A} \cap \bar{B}=\emptyset$.

Reciprocamente, suponha $K$ como no enunciado. Pelo corolário anterior, existe $h: \beta X \rightarrow$ $K$ contínua que fixa $X$ tal que $h[\beta X]=K$. Como $\beta X$ é compacto e $K$ é (em particular) Hausdorff, segue que $h$ é também uma aplicação fechada. Assim, resta apenas mostrarmos que $h$ é injetora. Para tanto, sejam $p$ e $q$ pontos distintos em $\beta X$ e tome $f: \beta X \rightarrow[0,1]$ contínua tal que $f(p)=0$ e $f(q)=1$ (o que podemos fazer pois $\beta X$ é um espaço de Tychonoff).

Agora, notemos que $A_{0}=\left\{x \in X: f(x) \leq \frac{1}{3}\right\}$ e $A_{1}=\left\{x \in X: f(x) \geq \frac{2}{3}\right\}$ são completamente separados em $X$ (basta notar que $A_{0}$ e $A_{1}$ são subconjuntos de fechados disjuntos em $\beta X$ e aplicar o Lema de Urysohn para tais fechados em $\beta X$, que é normal). Logo, a hipótese sobre $K$ nos diz que $A_{0}$ e $A_{1}$ têm fechos disjuntos em $K$. Como $h$ fixa $X$, temos $h(p) \in \overline{A_{0}}$ e $h(q) \in \overline{A_{1}}$, e consequentemente $h(p) \neq h(q)$.

Para espaços normais, o Lema de Urysohn nos garante que dois conjuntos fechados são disjuntos se, e somente se, forem completamente separados. Assim, o próximo corolário é imediato.

Corolário 3.1.14. Seja $X$ um espaço normal. Se $A, B \subseteq X$ são fechados disjuntos, então $A$ e $B$ têm fechos disjuntos em $\beta X$. Reciprocamente, se $K$ é uma compactificação de $X$ tal que $A$ e $B$ têm fechos disjuntos em $K$ sempre que $A$ e $B$ forem fechados disjuntos de $X$, então $K$ é homeomorfo a $\beta X$.

Corolário 3.1.15. Se $X$ é normal e $M \subseteq X$ é fechado, então $\bar{M}$ em $\beta X$ é homeomorfo a $\beta M$.

Demonstração. Como $M$ é fechado em $X$, segue que $M$ é normal. Agora, se $A$ e $B$ são fechados disjuntos em $M$, então $A$ e $B$ são fechados disjuntos em $X$, donde segue que ambos têm fechos disjuntos em $\beta X$ e, por conseguinte, $A$ e $B$ têm fechos disjuntos em $\bar{M}$. Pelo corolário anterior, concluímos que $\bar{M}$ é homeomorfo a $\beta M$.

O seguinte corolário é uma aplicação interessante dos resultados vistos até aqui, devido a Stone, [43]: 
Corolário 3.1.16 (Teorema de Tietze). Sejam $X$ um espaço normal e $A \subseteq X$ fechado e $K$ um compacto Hausdorff. Se $f: A \rightarrow K$ é contínua, então existe $\tilde{f}: X \rightarrow K$ contínua que estende $f$.

Demonstração. Como $A$ é fechado num espaço normal, o corolário anterior nos diz que $\bar{A}$ em $\beta X$ é homeomorfo a $\beta A$. Logo, existe $\bar{f}: \bar{A} \rightarrow K$ que estende $f$ continuamente. Agora, $\bar{f}$ é uma função contínua definida num compacto de $\beta X$, o qual é um espaço de Tychonoff. Logo, pelo Corolário 1.2.2 (que provamos sem o uso do Teorema de Tietze na Seção 1.2), existe uma extensão contínua $F: \beta X \rightarrow K$ para $\bar{f}$. Basta então definir $\tilde{f}=F \uparrow X$.

Exemplo 3.1.17. A compactificação de um ponto de $\mathbb{R}$ não é a compactificação de Stone-Čech de $\mathbb{R}$. De fato, para ver isto, considere $f: \mathbb{R} \rightarrow[0,1]$ a função dada por

$$
f(x)=\left\{\begin{array}{ccc}
-1 & \text {, se } & x \leq-1 \\
x & \text {, se } & -1<x<1 \\
1 & \text {, se } & 1 \leq x
\end{array} .\right.
$$

Note que $f$ não pode ter uma extensão contínua $F: \mathbb{R} \cup\{\infty\} \rightarrow[0,1]$, pois $\{-(n+1)$ : $n \in \omega\}$ e $\{n+1: n \in \omega\}$ convergem para $\infty$ em $\mathbb{R} \cup\{\infty\}$, o que acarretaria $F(\infty)=-1$ e $F(\infty)=1$.

Exemplo 3.1.18. O intervalo fechado $[0,1]$ não é a compactificação de Stone-Čech de $(0,1)$ e, por conseguinte, não é $\beta \mathbb{R}$. Com efeito, $g:(0,1) \rightarrow[-1,1]$ dada por $g(x)=\operatorname{sen}\left(\frac{1}{x}\right)$ não admite extensão contínua em $[0,1]$.

Exemplo 3.1.19. A compactificação de Stone-Čech de $\omega_{1}$ é $\omega_{1}+1$.

Demonstração. Claramente, $\omega_{1}$ é denso em $\omega_{1}+1$. Como $\omega_{1}+1$ é de Hausdorff, precisamos mostrar que $\omega_{1}+1$ é compacto e tem a propriedade da extensão de funções contínuas.

Fato 3.1.20. Um ordinal é sucessor se, e somente se, é compacto na topologia da ordem.

Prova. Suponha $\alpha=\gamma+1$, e seja $\mathcal{U}=\left\{U_{i}\right\}_{i \in I}$ uma cobertura por abertos para $\alpha$. Chame de $A=\{x \in \alpha:[0, x]$ está contido na reunião de finitos elementos de $\mathcal{U}\}$, o qual é não vazio pois $0 \in A$. Assuma $\alpha \backslash A \neq \emptyset$, e seja $x_{0}=\min \alpha \backslash A$. Como $\mathcal{U}$ é cobertura, existe $i \in I$ tal 
que $x_{0} \in U_{i}$ e, como $x_{0}>0, U_{i}$ deve conter $\left.] y, x_{0}\right]$, onde $y<x_{0}$. Consequentemente, $[0, y]$ está contido na reunião de finitos elementos de $\mathcal{U}$, digamos $U_{i_{0}}, \ldots, U_{i_{n}} \in \mathcal{U}$, e assim segue que $\left[0, x_{0}\right] \subseteq \bigcup_{j \leq n} U_{i_{j}} \cup U_{i}$, o que contraria $x_{0} \in \alpha \backslash A$. Logo, $\alpha=[0, \gamma]=A$ e, portanto, $\alpha$ é compacto.

Reciprocamente, se $\alpha$ é compacto, então $\{[0, x]\}_{x \in \alpha}$ é uma cobertura de abertos para $\alpha$ que possui subcobertura finita, digamos $\left[0, x_{0}\right], \ldots,\left[0, x_{n}\right]$. Note então que isto implica em $\alpha=$ $[0, w]=w+1$, onde $w=\max \left\{x_{0}, \ldots, x_{n}\right\}$.

Por fim, mostremos que toda função contínua $f: \omega_{1} \rightarrow[0,1]$ admite uma extensão contínua $F: \omega_{1}+1 \rightarrow[0,1]$. Para isto, provaremos que existe $\alpha<\omega_{1}$ tal que $f(\gamma)=f(\alpha)$ para todo $\gamma \geq \alpha$, e assim será suficiente fazer $F(\gamma)=f(\gamma)$ se $\gamma<\omega_{1}$ e $F\left(\omega_{1}\right)=f(\alpha)$. Vamos mostrar que para cada $n \in \omega$ existe $x_{n}<\omega_{1}$ tal que $\left|f(x)-f\left(x_{n}\right)\right|<\frac{1}{n+1}$ para todo $x \geq x_{n}$, e assim basta tomarmos $\alpha=\sup \left\{x_{n}: n \in \omega\right\}+1$ : de fato, se $\gamma \geq \alpha$, então

$$
|f(\gamma)-f(\alpha)| \leq\left|f(\gamma)-f\left(x_{n}\right)\right|+\left|f\left(x_{n}\right)-f(\alpha)\right|<\frac{2}{n+1}
$$

para todo $n \in \omega$, o que implica $f(\gamma)=f(\alpha)$.

Suponha então que exista $i \in \omega$ tal que para todo $x<\omega_{1}$ existe $x^{\prime}>x$ tal que $\mid f(x)-$ $f\left(x^{\prime}\right) \mid \geq \frac{1}{i}$. Tal suposição nos permite obter sequências $\left\{a_{n}: n \in \omega\right\},\left\{b_{n}: n \in \omega\right\} \subset \omega_{1}$ tais que $a_{n}<b_{n}<a_{n+1}$ e $\left|f\left(a_{n}\right)-f\left(b_{n}\right)\right| \geq \frac{1}{i}$ para todo $n \in \omega$. Como ambas as sequências são enumeráveis, segue que $c=\sup \left(\left\{a_{n}: n \in \omega\right\} \cup\left\{b_{n}: n \in \omega\right\}\right)<\omega_{1}$. Afirmamos que $f$ não é contínua em $c$ : de fato, note que se $V$ é uma vizinhança de $c$, então existe $y<c$ tal que ]$y, c] \subseteq V$ e, por conseguinte, existe $n \in \omega$ tal que $a_{m}, b_{m} \in V$ para todo $m \geq n$; por outro lado, $f^{-1}\left[\left(f(c)-\frac{1}{2 i}, f(c)+\frac{1}{2 i}\right)\right]=B$ não pode conter simultaneamente $a_{j}$ e $b_{j}$, qualquer que seja $j \in \omega$, acarretando que $B$ não é vizinhança de $c$ em $\omega_{1}$.

Algumas propriedades topológicas de $X$ permitem obter mais informações sobre $\beta X$. Quando $X$ é um discreto infinito, por exemplo, temos $|\beta X|=2^{2^{|X|}}$ e $w(\beta X)=2^{|X|}$. Para provar isso, demonstramos inicialmente o seguinte lema:

Lema 3.1.21. Para todo cardinal $\kappa \geq \aleph_{0}, w\left([0,1]^{\kappa}\right)=\kappa$. 
Demonstração. Já temos $w\left([0,1]^{\kappa}\right) \leq w([0,1]) \cdot \kappa=\kappa$. Para obter a outra desigualdade, vamos obter um subespaço discreto $D$ de $[0,1]^{\kappa}$, tal que $|D|=\kappa$; como $\kappa=w(D) \leq w\left([0,1]^{\kappa}\right)$, a desigualdade estará provada. Considere $D$ como sendo o cardinal $\kappa$ munido da topologia discreta e, para cada $\alpha<\kappa$, defina $f_{\alpha}:=\chi_{\{\alpha\}}: D \rightarrow[0,1]$ a função (contínua) característica em $\{\alpha\}$. Como $D$ é normal (em particular $T_{1}$ ) e $\mathcal{F}=\left\{f_{\alpha}: \alpha<\kappa\right\}$ separa pontos de fechados em $D$, segue pelo Teorema da Diagonal que

$$
\Delta_{\alpha<\kappa} f_{\alpha}: D \rightarrow \prod_{\alpha<\kappa}[0,1]=[0,1]^{\kappa}
$$

é um mergulho.

Teorema 3.1.22 (Pospíšil, apud Engelking [15], Teorema 3.6.11). Se D é um discreto infinito, então $|\beta D|=2^{2^{|D|}}$ e $w(\beta D)=2^{|D|}$.

Demonstração. Seja $|D|=\kappa \geq \aleph_{0}$. Primeiramente, note que $K=[0,1]^{2^{\kappa}}$ é um espaço compacto de cardinalidade $2^{2^{\kappa}}$ e $w(K)=2^{\kappa}$ pelo lema anterior. Assim, da definição de $\beta D$, temos $\beta D \subseteq[0,1]^{[0,1]^{D}}$, que é homeormofo a $K$, donde segue que $|\beta D| \leq 2^{2^{\kappa}}$ e $w(\beta D) \leq 2^{\kappa}$.

Agora, pelo Teorema de Hewitt-Marczewiski-Pondiczery ( $c f$. Teorema 2.2.13), $K$ contém um denso $A$ de cardinalidade $\kappa$. Assim, existe $f: D \rightarrow K$ tal que $f[D]=A$ e, pelo Teorema 3.1.4 existe uma extensão contínua $\beta f: \beta D \rightarrow K$; note que $\beta f[\beta D]=K$, pois $\beta f[\beta D]$ é um fechado de $K$ (pois $K$ é Hausdorff, $\beta D$ é compacto e $\beta f$ é contínua) que contém o denso $A$. Logo, $|\beta D| \geq|K|=2^{2^{\kappa}}$. Resta apenas verificar que $2^{\kappa} \leq w(\beta D)$. Como $\beta f$ é sobrejetora e contínua, se $\mathfrak{B}$ for uma base para $\beta D$, então $\{\beta f[B]: B \in \mathfrak{B}\}$ é uma network em $K$, o que mostra $n w(K) \leq w(\beta D)$. Mas como $K$ é compacto, temos $n w(K)=w(K)=2^{\kappa}$.

Corolário 3.1.23. $|\beta \omega|=2^{\mathfrak{c}}$ e $w(\beta \omega)=\mathfrak{c}$.

Corolário 3.1.24. Se X é um espaço normal que contém um discreto fechado infinito $D$, então $|\beta X| \geq 2^{2^{|D|}}$ e $w(\beta X) \geq 2^{|D|}$. Em particular, $|\beta \mathbb{R}|=2^{\mathfrak{c}}$.

Demonstração. A primeira afirmação decorre do fato de que $\bar{D}$ em $\beta X$ é homeomorfo a $\beta D$. Para a segunda afirmação, note que como $\mathbb{Q}$ é enumerável, existe $f: \omega \rightarrow \mathbb{Q}$ bijetora. Logo, $\beta f: \beta \omega \rightarrow \beta \mathbb{Q}$ estende $f$ continuamente e é tal que $\beta f[\beta \omega]=\beta \mathbb{Q}$, pois

$$
\beta \mathbb{Q}=\overline{\mathbb{Q}} \subseteq \overline{\beta f[\beta \omega]}=\beta f[\beta \omega] \subseteq \beta \mathbb{Q}
$$


o que nos dá $|\beta \mathbb{Q}| \leq|\beta \omega|$. Finalmente, a inclusão $i: \mathbb{Q} \rightarrow \mathbb{R} \subset \beta \mathbb{R}$ tem uma extensão contínua $\beta i: \beta \mathbb{Q} \rightarrow \beta \mathbb{R}, \mathrm{e}$

$$
\beta \mathbb{R}=\overline{\mathbb{R}}=\overline{\mathbb{Q}} \subseteq \overline{\beta i[\beta \mathbb{Q}]}=\beta i[\beta \mathbb{Q}] \subseteq \beta \mathbb{R}
$$

donde segue que $|\beta \mathbb{R}| \leq|\beta \mathbb{Q}|$.

Em particular, não apenas $|\beta \omega|=2^{\mathfrak{c}}$, mas todo subespaço fechado de $\beta \omega$ também tem cardinalidade $2^{\mathfrak{c}}$, como provamos no seguinte teorema:

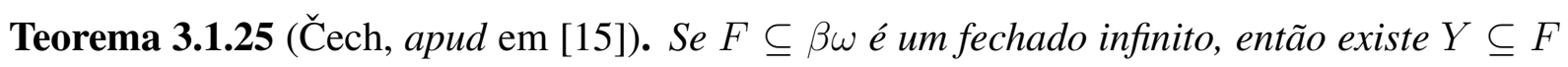
homeomorfo a $\beta \omega$.

Demonstração. Primeiramente, lembremo-nos do seguinte fato.

Fato 3.1.26. Sejam $(K, \tau)$ um espaço compacto e $Y \subseteq K$. Se $Y$ é infinito, então $Y$ possui um ponto de acumulação.

Prova. Procedemos pela contrapositiva. Suponha que para todo $x \in K$ exista $V_{x} \in \tau$ tal que $x \in V_{x}$ e $V_{x} \cap(Y \backslash\{x\})=\emptyset$. Da compacidade de $K$, existem $x_{0}, \ldots, x_{n} \in K$ tais que $K=\bigcup_{i \leq n} V_{x_{i}}$. Em particular, temos $V_{x_{i}} \cap\left(Y \backslash\left\{x_{i}\right\}\right)=\emptyset$ para todo $i \leq n$. Assim, se $x \in Y$, então existe $i \leq n$ tal que $x \in V_{x_{i}}$ e, por conseguinte, $V_{x_{i}} \cap Y \neq \emptyset$, donde segue que $x=x_{i}$ e, portanto, $Y \subseteq\left\{x_{0}, \ldots, x_{n}\right\}$.

Pelo fato acima, existe $b \in F$ um ponto de acumulação de $F$. Vamos obter uma família infinita $A=\left\{a_{i}: i \in \omega\right\} \subseteq F \backslash\{b\}$ discreta e, portanto, homeomorfa a $\omega$. Seja $a_{0} \in F \backslash\{b\}$. Como $\beta \omega$ é de Hausdorff, existem abertos disjuntos $V_{0}, U_{0}$ tais que $a_{0} \in V_{0}$ e $b \in U_{0}$. Suponha definidos $a_{i} \in F \backslash\{b\}$ e $V_{i}, U_{i} \subseteq \beta \omega$ abertos, para $i \leq n$, tais que
(a) $a_{i} \in V_{i}, \forall i \leq n$;
(b) $b \in U_{i}, \forall i \leq n$;
(c) $V_{i} \cap V_{j}=\emptyset$ se $i \neq j$;
(d) $V_{i} \cap U_{i}=\emptyset \forall i \leq n$;
(e) $U_{i} \subseteq U_{j}$ se $j \leq i$. 
Vamos obter $a_{n+1} \in F \backslash\{b\}$ e abertos $V_{n+1}, U_{n+1}$ satisfazendo as condições acima. Como $b$ é ponto de acumulação, existe $a_{n+1} \in U_{n} \cap(F \backslash\{b\})$ e, por conseguinte, existem $V, U$ abertos disjuntos de $\beta \omega$ tais que $a_{n+1} \in V$ e $b \in U$. Por fim, chame $U_{n+1}=U \cap U_{n}$ e $V_{n+1}=V \cap U_{n}$ (veja a ilustração abaixo). É fácil ver que as condições impostas ainda são satisfeitas pela terna $\left(a_{n+1}, V_{n+1}, U_{n+1}\right)$ obtida.

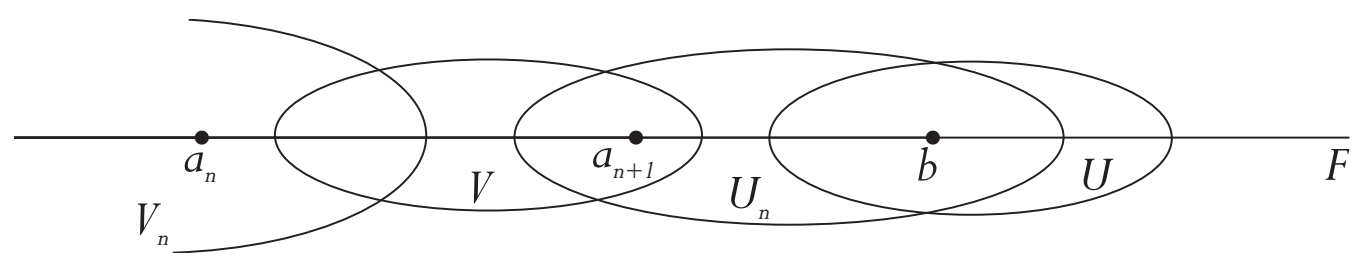

Agora, considere $g: A \rightarrow[0,1]$ uma função (contínua, pois $A$ é discreto), e defina $g_{0}: \omega \rightarrow$ $[0,1]$ por

$$
g_{0}(n)=\left\{\begin{array}{cc}
g\left(a_{i}\right), & \text { se } n \in \omega \cap V_{i} \\
0, & \text { se } n \in \omega \backslash \bigcup_{i \in \omega} V_{i}
\end{array} .\right.
$$

Como $g_{0}$ é contínua, existe $\beta g_{0}: \beta \omega \rightarrow[0,1]$ contínua que estende $g_{0}$. Finalmente, afirmamos que $\beta g_{0} \uparrow A=g$. De fato, como $\omega$ é denso em $\beta \omega$, temos $\overline{V_{i}}=\overline{V_{i} \cap \omega}$; além disso, como para qualquer função contínua $f$ vale $f[\bar{A}] \subseteq \overline{f[A]}$, para $a_{i} \in A$ temos

$$
\beta g_{0}\left(a_{i}\right) \in \beta g_{0}\left[V_{i}\right] \subseteq \beta g_{0}\left[\overline{V_{i}}\right]=\beta g_{0}\left[\overline{V_{i} \cap \omega}\right] \subseteq \overline{\beta g_{0}\left[V_{i} \cap \omega\right]}=\overline{\left\{g\left(a_{i}\right)\right\}}=\left\{g\left(a_{i}\right)\right\}
$$

isto é, $\beta g_{0}\left(a_{i}\right)=g\left(a_{i}\right)$. Dessa forma, temos $\bar{A} \subseteq F$ homeomorfo a $\beta A$ e, como $A$ é discreto enumerável, temos $\beta A$ homeomorfo a $\beta \omega$.

Corolário 3.1.27. Seja $X=\left\{x_{n}: n \in \omega\right\} \cup\{x\} \subseteq \beta \omega$. Se $x_{n} \rightarrow x$, então $|X|<\aleph_{0}$, isto é, as únicas sequências convergentes de $\beta \omega$ são as quase constantes.

Demonstração. Se $x_{n} \rightarrow x$, então $F=\left\{x_{n}\right\}_{n \in \omega} \cup\{x\}$ é um compacto de $\beta \omega$ e, por conseguinte, é fechado em $\beta \omega$. Logo, se $F$ fosse infinito, teríamos pelo teorema anterior que $|F|>\aleph_{0}$, o que não ocorre. 


\section{2 $P$-pontos em $\beta \omega \backslash \omega$ na presença de $\mathbf{C H}$}

Para um espaço topológico $X$, dizemos que $x \in X$ é um P-ponto se toda interseção enumerável de vizinhanças de $x$ contém uma vizinhança de $x$. Note que se $X$ é um espaço compacto Hausdorff no qual todos os seus pontos são $P$-pontos, então $X$ é necessariamente finito. De fato, se $G$ é um $G_{\delta}$ não vazio de $X$, então $G$ é aberto: dado $x \in G$, por $x$ ser um $P$-ponto segue que existe uma vizinhança de $x$ contida em $G$. Em virtude disso, toda reunião enumerável de fechados é fechada em $X$ e, já que $X$ é de Hausdorff, se $X$ fosse infinito poderíamos obter um subconjunto de $X$, infinito enumerável e discreto, e assim existiria um discreto enumerável e fechado, o que não pode ocorrer num compacto ( $c f$. Fato 3.1.26).

Em [37], Rudin mostrou que a validade de CH implica a existência de $P$-pontos em $\omega^{*}(=$ $\beta \omega \backslash \omega)$ e, consequentemente, provou que ao assumirmos $\mathrm{CH}, \omega^{*}$ não é um espaço homogêned ${ }^{1}$, como $\omega^{*}$ é infinito, deve haver pelo menos um ponto $x$ de $\omega^{*}$ que não é $P$-ponto, mas como existe pelo menos um $P$-ponto $y$, segue que não existe homeomorfismo que leva $x$ em $y$.

Nesta seção, apresentaremos os métodos utilizados por Rudin para mostrar a existência de $P$-pontos em $\omega^{*}$ ao assumirmos a Hipótese do Contínud ${ }^{2}$, Ao leitor interessado em outras consequências de $\mathrm{CH}$ no que tange a $\beta \omega$, recomendamos o artigo de Jan van Mill [33], no qual $\beta \omega$ é estudado em $\mathrm{ZFC}, \mathrm{ZFC}+\mathrm{CH}$ e $\mathrm{ZFC}+\neg \mathrm{CH}$.

Primeiramente, vamos introduzir alguns conceitos de Álgebras Booleanas - os resultados não demonstrados podem ser encontrados nos capítulos 1 e 3 de [30]. Uma álgebra Booleana é uma 6-upla $(A, \oplus, \odot, \ominus, 0,1)$, onde $A$ é um conjunto, 0 e 1 são elementos de $A, \oplus: A \times A \rightarrow A$ e $\odot: A \times A \rightarrow A$ e $\ominus: A \rightarrow A$ são operações satisfazendo as seguintes condições:

(a) associatividade: $x \oplus(y \oplus z)=(x \oplus y) \oplus z \mathrm{e} x \odot(y \odot z)=(x \odot y) \odot z$;

(b) comutatividade: $x \oplus y=y \oplus x \mathrm{e} x \odot y=y \odot x$;

(c) absorção: $x \oplus(x \odot y)=x$ e $x \odot(x \oplus y)=x$;

\footnotetext{
${ }^{1}$ Embora a não homogeneidade de $\omega^{*}$ já esteja estabelecida em ZFC ( $c f$. Walker [49], Capítulo 4), é consistente que $\omega^{*}$ não admita $P$-pontos (resultado devido a Shelah, apud van Mill [33]).

${ }^{2}$ Para o leitor familiarizado com pequenos cardinais ( $c f$. Seção 4.3 , $\mathfrak{p}=\mathfrak{c}$ é suficiente para garantir a existência de $P$-pontos em $\omega^{*}$. Tanto a definição do cardinal $\mathfrak{p}$ quanto a demonstração do resultado supracitado podem ser encontrados, por exemplo, em [42].
} 
(d) distributividade: $x \odot(y \oplus z)=(x \odot y) \oplus(x \odot z)$ e $x \oplus(y \odot z)=(x \odot y) \oplus(x \odot z)$;

(e) complementação: $x \oplus(\ominus x)=1$ e $x \odot(\ominus x)=0$.

Quando o contexto tornar claro, diremos simplesmente que $A$ é uma álgebra Booleana. Além disso, geralmente denotaremos $\oplus, \odot \mathrm{e} \ominus$ respectivamente por,$+ \cdot \mathrm{e}-$. Um exemplo natural de álgebra Booleana se dá ao considerarmos $\wp(X)$ munido das operações $\cup, \cap$, $\backslash$, que correspondem respectivamente $\mathrm{a}+, \cdot,-$; claramente, $\emptyset \in \wp(X)$ e $X \in \wp(X)$ correspondem a 0 e 1 , respectivamente.

Consideremos uma álgebra Booleana fixada $A$. Podemos dotar $A$ de uma ordem parcial $\leq$ declarando, para quaisquer $x, y \in A$,

$$
x \leq y \Leftrightarrow x+y=y
$$

note que $x+y=y$ é equivalente a $x \cdot y=x$. Além disso, 1 e 0 são, respectivamente, o máximo e o mínimo de $A$ com respeito à ordem $\leq$.

Tal estrutura de ordem em $A$ nos permite analisar os filtros e ultrafiltros em $A$. Dizemos que $F \subseteq A$ é um filtro (próprio) se $F$ satisfaz as seguintes condições:

(a) $1 \in F$ e $0 \notin F$;

(b) $x, y \in F \Rightarrow x \cdot y \in F$;

(c) $y \geq x \in F \Rightarrow y \in F$.

Em particular, quando $E \subseteq A$, então $\langle E\rangle:=\left\{x \in A: \exists n \in \omega \wedge \exists e_{0}, \ldots, e_{n} \in E\left(e_{0} \cdot \ldots \cdot e_{n} \leq\right.\right.$ $x)\}$ é o menor (no sentido da inclusão) filtro em $A$ que contém $E$. Além disso, $\langle E\rangle$ é próprio se, e somente se, $E$ tem a propriedade da interseção finita (p.i.f), isto é, se para qualquer subcoleção finita $\left\{x_{0}, \ldots, x_{n}\right\} \subseteq E$ valer $x_{0} \cdot \ldots \cdot x_{n} \neq 0$. Pelo Lema de Zorn, é fácil ver que se $E$ tem a p.i.f., então existe um filtro próprio $F$ que é maximal (com respeito a inclusão) e contém $E$. Chamamos um filtro próprio $F$ em $A$ de ultrafiltro se $F$ é maximal com respeito à inclusão. Frisamos que são equivalentes:

(a) $F$ é ultrafiltro em $A$; 
(b) para todo $a \in A, a \in F$ se, e somente se, $-a \notin F$;

(c) se $x, y \in A$ são tais que $x+y \in F$, então $x \in F$ ou $y \in F$.

Denotamos por Ult $(A)$ a coleção de todos os ultrafiltros em $A$ e, para cada $a \in A$, definimos $s(a):=\{\mathfrak{u} \in \operatorname{Ult}(A): a \in \mathfrak{u}\}$. Note que se $a, b \in A$, então $s(a) \cap s(b)=s(a \cdot b)$ : claramente, $\mathfrak{u} \in s(a) \cap s(b)$ implica em $\mathfrak{u} \in s(a \cdot b)$, por outro lado, se $a \cdot b \in \mathfrak{u}$ e $a, b \notin \mathfrak{u}$, obtemos $-a,-b \in \mathfrak{u}$, o que nos dá $(-a) \cdot(-b) \in \mathfrak{u}$ e, consequentemente, $0=(a \cdot b) \cdot((-a) \cdot(-b)) \in \mathfrak{u}$, o que não pode acontecer. Como todo ultrafiltro pertence a algum conjunto da forma $s(a)$, vemos que $s[A]:=\{s(a): a \in A\}$ é base para uma topologia $\mathscr{T}_{A}$ em Ult $(A)$; chamamos $\left(\mathrm{Ult}(A), \mathscr{T}_{A}\right)$ de espaço de Stone de $A$, o qual denotamos por $s(A)$.

Um espaço topológico $X$ é zero-dimensional se admite uma base de abertos-fechados; quando, além disso, $X$ é também um compacto Hausdorff, dizemos que $X$ é um espaço Booleano.

Proposição 3.2.1. Se A é uma álgebra Booleana, então s $(A)$ é um espaço Booleand ${ }^{3}$

Demonstração. Se u é um ultrafiltro de $A$, então $a \notin \mathfrak{u} \Leftrightarrow-a \in \mathfrak{u}$, isto é, $\operatorname{Ult}(A) \backslash s(a)=$ $s(-a)$, donde segue que $s(A)$ é zero-dimensional. Além disso, se $\mathfrak{u}$ e q são ultrafiltros distintos, então existe $u \in \mathfrak{u} \backslash \mathfrak{q}$, acarretando $\mathfrak{u} \in s(u)$ e $\mathfrak{q} \in s(-u)$, com $s(u) \cap s(-u)=\emptyset$, donde segue que $s(A)$ é de Hausdorff. Resta mostrar a compacidade.

Sejam $A^{\prime} \subseteq A$ e $\mathcal{U}=\left\{s(a): a \in A^{\prime}\right\}$ uma cobertura por abertos para Ult $(A)$. Se $\mathcal{U}$ não admite subcobertura finita, então para cada $n \in \omega$ e $a_{0}, \ldots, a_{n} \in A^{\prime}$ temos

$$
s\left(a_{0}\right) \cup \cdots \cup s\left(a_{n}\right) \neq \mathrm{UIt}(A)=s(1),
$$

donde segue que $-a_{0} \cdot \ldots \cdot-a_{n} \neq 0$, pois $-a_{0} \cdot \ldots \cdot-a_{n}=-\left(a_{0}+\cdots+a_{n}\right)$. Desse modo, o conjunto $-A^{\prime}:=\left\{-a: a \in A^{\prime}\right\}$ tem p.i.f. e, portanto, existe $\mathfrak{u} \in \operatorname{Ult}(A)$ que contém $-A^{\prime}$ e, assim, $-a \in \mathfrak{u}$ para todo $a \in A^{\prime}$, o que nos dá $a \notin \mathfrak{u}$ para todo $a \in A^{\prime}$, isto é, $\mathfrak{u} \notin s(a)$, contrariando o fato de que $\mathcal{U}$ é uma cobertura.

A partir daqui, nos focaremos na álgebra Booleana $A=\wp(\omega)$ dos subconjuntos de $\omega$. Começamos por identificar o espaço dual de $\wp(\omega)$, no seguinte teorema.

\footnotetext{
${ }^{3}$ A recíproca também é verdadeira, no seguinte sentido: todo espaço Booleano é o espaço de Stone de uma álgebra Booleana ( $c f$. [30], Teorema 7.10).
} 
Teorema 3.2.2. $s(\wp(\omega))$ é a compactificação de Stone- $\breve{C} e c h$ de $\omega$.

Demonstração. Primeiramente, vamos "encontrar" $\omega$ em $s(\wp(\omega))$. Afirmamos que $\omega$ é homeomorfo a $\mathcal{N}=\left\{\mathfrak{u}_{n}: n \in \omega\right\}$, onde $\mathfrak{u}_{n}=\{A \subseteq \omega: n \in A\} \in \mathrm{Ult}(\omega)$. Para verificar isto, basta notar que $\mathcal{N}$ é infinito e discreto em $s(\wp(\omega))$ : se $m \neq n$ são naturais, então $\mathfrak{u}_{n} \neq \mathfrak{u}_{m}$ e, para qualquer $n \in \omega, s(\{n\})=\left\{\mathfrak{u}_{n}\right\}$. Agora, como $s(\wp(\omega))$ é um compacto Hausdorff, para concluir o teorema basta mostrarmos que $\mathcal{N}$ é denso em $s(\wp(\omega))$ e que toda função $f: \mathcal{N} \rightarrow[0,1]$ admite extensão contínua $g: s(\wp(\omega)) \rightarrow[0,1]$.

$\underline{\mathcal{N} \text { é denso em } s(\wp(\omega))}$. Se $s(a) \neq \emptyset$ é um aberto básico de $s(\wp(\omega))$, temos necessariamente $a \subseteq \omega$ não vazio, e assim existe $n \in a$, o que acarreta $a \in \mathfrak{u}_{n}$ e, por conseguinte, $\mathfrak{u}_{n} \in s(a)$.

Extensão de funções. Fixemos $f: \mathcal{N} \rightarrow[0,1]$ uma função (contínua, pois $\mathcal{N}$ é discreto). Para cada $A \in \wp(\omega)$, defina $A^{\prime}=\left\{\mathfrak{u}_{n}: n \in A\right\}$. Afirmamos que, para cada ultrafiltro $\mathfrak{u} \in s(\wp(\omega))$, a interseção $G(\mathfrak{u})=\bigcap_{A \in \mathfrak{u}} \overline{f\left[A^{\prime}\right]}$ é um conjunto unitário. É claro que $G(\mathfrak{u}) \neq \emptyset$, pois $\mathfrak{u}$ tem p.i.f. (por ser filtro), o que faz de $\left\{\overline{f\left[A^{\prime}\right]}\right\}_{A \in \mathfrak{U}}$ uma família de fechados de $[0,1]$ com p.i.f., e a compacidade de $[0,1]$ nos permite então concluir que $G(\mathfrak{u}) \neq \emptyset$.

Se $G(\mathfrak{u})$ não fosse unitário, existiriam $x, y \in G(\mathfrak{u}) \operatorname{com}|x-y|>r>0$ para algum $r \in \mathbb{R}$ e, assim, $B=\left\{n \in \omega:\left|f\left(\mathfrak{u}_{n}\right)-x\right|<\frac{r}{2}\right\} \notin\{\emptyset, \omega\}(B \neq \emptyset$ pois $x \in G(\mathfrak{u})$ e $B \neq \omega$ pois $y \in G(\mathfrak{u})$ ). Como $\mathfrak{u}$ é ultrafiltro, temos necessariamente uma dentre as duas possibilidades: $B \in \mathfrak{u}$ ou $\omega \backslash B \in \mathfrak{u}$. Contudo, $x \notin \overline{f\left[(\omega \backslash B)^{\prime}\right]}$ e $y \notin \overline{f\left[B^{\prime}\right]}$. Deste modo, para cada $\mathfrak{u} \in s(\wp(\omega))$, temos $G(\mathfrak{u})=\{g(\mathfrak{u})\}$, para algum $g(\mathfrak{u}) \in[0,1]$, o que nos permite definir

$$
\begin{aligned}
g: s(\wp(\omega)) & \rightarrow[0,1] \\
\mathfrak{u} & \mapsto g(\mathfrak{u})
\end{aligned}
$$

Note que $g\left\lceil\mathcal{N}=f\right.$, já que se $A \in \mathfrak{u}_{n}$, então $n \in A$ e, por conseguinte, $\mathfrak{u}_{n} \in A^{\prime}$, o que acarreta $f\left(\mathfrak{u}_{n}\right) \in f\left[A^{\prime}\right] \subseteq \overline{f\left[A^{\prime}\right]}$ para todo $A \in \mathfrak{u}_{n}$, mostrando que $f\left(\mathfrak{u}_{n}\right) \in G\left(\mathfrak{u}_{n}\right)$. Resta apenas verificar que $g$ é contínua.

Sejam $\mathfrak{u} \in s(\wp(\omega)), \varepsilon>0$ e consideremos $B=\left\{n \in \omega:\left|g(\mathfrak{u})-f\left(\mathfrak{u}_{n}\right)\right|<\frac{\varepsilon}{2}\right\}$. Necessariamente devemos ter $B \in \mathfrak{u}$, pois do contrário $\omega \backslash B \in \mathfrak{u}$, e $g(\mathfrak{u}) \notin \overline{f\left[(\omega \backslash B)^{\prime}\right]}$; assim, $s(B)$ é um 
aberto-fechado que contém $\mathfrak{u}$. Enfim, se $\mathfrak{v} \in s(B)$, então $B \in \mathfrak{v}$, e assim

$$
g(\mathfrak{v}) \in \bigcap_{A \in \mathfrak{v}} \overline{f\left[A^{\prime}\right]} \subseteq \overline{f\left[B^{\prime}\right]} \subseteq\left[g(\mathfrak{u})-\frac{\varepsilon}{2}, g(\mathfrak{u})+\frac{\varepsilon}{2}\right]
$$

Os mesmos argumentos se aplicam a um espaço discreto $D$ infinito, o que nos permite concluir que $s(\wp(D))$ é homeomorfo a $\beta D$. Em particular, para todo discreto infinito $D, \beta D$ é um espaço Booleano. Como $\omega$ é aberto em $\beta \omega$, segue que $\omega^{*}$ é fechado em $\beta \omega$ e, por conseguinte, é um compacto Hausdorff - chamaremos os elementos de $\omega^{*}$ de ultrafiltros livres (note que $\mathfrak{u} \in \omega^{*}$ se, e somente se, $\mathfrak{u} \neq \mathfrak{u}_{n}$ para todo $\left.n \in \omega\right)$.

Proposição 3.2.3. Se $a \subset \omega$ for finito e $a \in \mathfrak{u} \in s(\wp(\omega))$, então $\mathfrak{u}=\mathfrak{u}_{n}$ para algum $n \in$ a. Em particular, ultrafiltros livres não contém subconjuntos finitos de $\omega$.

Demonstração. Chame $a=\left\{x_{0}, \ldots, x_{m}\right\}$ e suponha que $\mathfrak{u} \neq \mathfrak{u}_{n}$ para todo $n \in a$. Como $\mathfrak{u}$ é ultrafiltro, segue que $\omega \backslash\left\{x_{i}\right\} \in \mathfrak{u}$ para cada $i \leq m$. Enfim, por termos $a \in \mathfrak{u}$, segue que $\emptyset=a \cap\left(\bigcap_{i \leq m} \omega \backslash\left\{x_{i}\right\}\right) \in \mathfrak{u}$, o que contraria o fato de $\mathfrak{u}$ ser próprio.

Como os conjuntos $s(a)=\{\mathfrak{u} \in \operatorname{UIt}(\wp(\omega)): a \in \mathfrak{u}\}$ formam uma base de abertos-fechados para $\beta \omega$, segue que os conjuntos $s^{*}(a):=\omega^{*} \cap s(a)$ constituem uma base de abertos para a topologia de $\omega^{*}$. Além disso, note que $s^{*}(a)=s^{*}(b)$ se, e somente se, $(a \backslash b) \cup(b \backslash a)$ for finito.

De fato, se $a \backslash b$ é finito, então $s^{*}(a) \subseteq s^{*}(b)$, pois do contrário existiria um ultrafiltro livre $\mathfrak{u}$ com $a \in \mathfrak{u}$ e $\omega \backslash b \in \mathfrak{u}$, mas isto daria $a \backslash b \in \mathfrak{u}$, o que viola a conclusão da proposição anterior; logo, se $(a \backslash b) \cup(b \backslash a)$ for finito, então $s^{*}(a) \subseteq s^{*}(b) \subseteq s^{*}(a)$. Reciprocamente, se $a \backslash b$ é infinito, então existe ${ }^{4}$ um ultrafiltro $\mathfrak{v}$ livre que contém $a$ e $a \backslash b$, o que acarreta $b \notin \mathfrak{v}$ e, portanto, $s^{*}(a) \not \subset s^{*}(b)$.

Tal argumentação nos permite concluir que $\mathcal{W}=\left\{s^{*}(a): a \in \wp(\omega) \backslash[\omega]^{<\omega}\right\}$ é uma base não enumerável para $\omega^{*}-$ mais precisamente, $\aleph_{1} \leq|\mathcal{W}| \leq \mathfrak{c}$. A próxima proposição nos dará o restante das informações necessárias sobre $\omega^{*}$ a fim de provarmos a existência de $P$-pontos.

Proposição 3.2.4. (a) $\omega^{*}$ é um espaço Booleano.

\footnotetext{
${ }^{4}$ Por meio do Axioma da Escolha, podemos garantir que existe um ultrafiltro que contém o filtro $\{c \subseteq \omega$ : $a \backslash b \subseteq c\}$.
} 
(b) Todo aberto-fechado de $\omega^{*}$ é da forma $s^{*}(a)$, para algum a $\subseteq \omega$ infinito.

(c) Todo $G_{\delta}$ não vazio de $\omega^{*}$ tem interior não vazio.

(d) Se A, B são abertos-fechados não vazios e distintos contidos propriamente em $\omega^{*}$, então existe um homeomorfismo de $\beta \omega$ que leva A sobre B, i.e., abertos-fechados próprios de $\omega^{*}$ são homeomorfos entre si.

Demonstração. Já sabemos que $\left\{s^{*}(a): a \in \wp(\omega) \backslash[\omega]<\omega\right\}$ é uma base de abertos para $\omega^{*}$, que é compacto Hausdorff. Assim, (a) segue facilmente por valer $\omega^{*} \backslash s^{*}(a)=s^{*}(\omega \backslash a)$.

Para o item $(b)$, considere $U \subseteq \omega^{*}$ um aberto-fechado não vazio e tome $f: \omega^{*} \rightarrow[0,1]$ a função característica de $U$, que é contínua. Como $\omega^{*}$ é fechado em $\beta \omega$, que por sua vez é um espaço normal, existe uma extensão contínua $\tilde{f}: \beta \omega \rightarrow[0,1]$ de $f$. Definimos então o conjunto $A=\left\{n \in \omega: \tilde{f}\left(\mathfrak{u}_{n}\right) \geq \frac{1}{2}\right\}$ e afirmamos que $U \subseteq s^{*}(A)$ : de fato, para $\mathfrak{u} \in U$, temos $\tilde{f}(\mathfrak{u})=1$ e, pela continuidade, existe $a \subseteq \omega$ infinito tal que $\mathfrak{u} \in s(a)$ e $\left.\tilde{f}[s(a)] \subseteq] \frac{1}{2}, 1\right]$ e, consequentemente, $A \in \mathfrak{u}$ (pois $a \in \mathfrak{u}$ e $a \subseteq A$ ). Analogamente, mostra-se que $\omega^{*} \backslash U \subseteq s^{*}(\omega \backslash A)$. Enfim, como $\omega^{*}=s^{*}(A) \sqcup s^{*}(\omega \backslash A)$, temos $U=s^{*}(A)$.

Provemos o item $(c)$. Seja $G=\bigcap_{n \in \omega} G_{n}$ um $G_{\delta}$ não vazio de $\omega^{*}$. Sem perda de generalidade, para cada $n$ podemos fazer $G_{n}=s^{*}\left(a_{n}\right)$ para algum $a_{n} \subseteq \omega$ infinito. Note que, para todo $n \in \omega, \bigcap_{i \leq n} a_{n}$ é infinito, pois $\bigcap_{i \leq n} s^{*}\left(a_{i}\right)=s^{*}\left(\bigcap_{i \leq n} a_{i}\right) \neq \emptyset$, e isso permite escolher $x_{n} \in \bigcap_{i \leq n} a_{n}$ tal que $x_{n} \neq x_{m}$ se $m \neq n$. Fazendo $A=\left\{x_{n}: n \in \omega\right\}$, para todo $i \in \omega$ temos $\left|A \backslash a_{i}\right|<\aleph_{0}$ por construção, o que nos dá $s^{*}(A) \subseteq s^{*}\left(a_{i}\right)$ e, por conseguinte, $s^{*}(A) \subseteq \bigcap_{n \in \omega} s^{*}\left(a_{n}\right)$.

Finalmente, provaremos o item $(d)$. Sejam $s^{*}(a)=A$ e $s^{*}(b)=B$ os abertos-fechados do enunciado. Como ambos são não vazios, decorre que tanto $a$ quanto $b$ são subconjuntos infinitos de $\omega$; por serem próprios, temos $\omega \backslash a$ e $\omega \backslash b$ infinitos. Logo, existe uma bijeção $f: \mathcal{N} \rightarrow \mathcal{N}$ tal que $f\left[a^{\prime}\right]=b^{\prime}-$ onde $a^{\prime}=\left\{\mathfrak{u}_{n}: n \in a\right\}$ e $b^{\prime}=\left\{\mathfrak{u}_{n}: n \in b\right\}$. Então $\beta f: \beta \omega \rightarrow \beta \omega$ é um homeomorfismo que satisfaz a condição imposta por $(d)$. 
Teorema 3.2.5 (Rudin, [37], Teorema 4.2). (CH) Existe pelo menos um P-ponto em $\omega^{*}$. Além disso, o conjunto dos P-pontos de $\omega^{*}$ é denso.

Demonstração. Como a Hipótese do Contínuo afirma que $\mathfrak{c}=\aleph_{1}$, temos

$$
\mathcal{W}=\left\{s^{*}(a): a \in \wp(\omega) \backslash[\omega]^{<\omega}\right\}=\left\{W_{\alpha}\right\}_{\alpha<\omega_{1}},
$$

e não há perda de generalidade em supor $W_{0}=\omega^{*}$. Vamos obter uma família $\mathcal{A}=\left\{A_{\alpha}\right\}_{\alpha<\omega_{1}}$ de abertos-fechados não vazios e decrescente com respeito à inclusão, donde seguirá que $\mathcal{A}$ será uma família de fechados com p.i.f. em $\omega^{*}$, e a compacidade de $\omega^{*}$ nos dará $\bigcap \mathcal{A} \neq \emptyset$; em verdade, $\bigcap \mathcal{A}$ conterá exatamente um único ponto, o qual provaremos ser um $P$-ponto.

Chame $A_{0}=W_{0}$ e para $\alpha<\omega_{1}$, suponha definidos abertos-fechados $A_{\beta}$ para cada $\beta<\alpha$, tais que $B_{\alpha}=\bigcap_{\beta<\alpha} A_{\beta} \neq \emptyset$. Como $\alpha$ é enumerável, temos $B_{\alpha}$ um $G_{\delta}$ não vazio de $\omega^{*} \mathrm{e}$, pelo item $(c)$ da proposição anterior, inferimos a existência de um aberto-fechado não vazio $A^{\prime} \subseteq B_{\alpha}$. Chame $A_{\alpha}=A^{\prime}$ se $A^{\prime} \cap W_{\alpha}=\emptyset$, caso contrário faça $A_{\alpha}=A^{\prime} \cap W_{\alpha}$.

Por construção, $\mathcal{A}=\left\{A_{\alpha}\right\}_{\alpha<\omega_{1}}$ é uma família com p.i.f. e, da compacidade de $\omega^{*}$, segue que existe $\mathfrak{u} \in \bigcap \mathcal{A}$. Como $\mathcal{W}$ é uma base para $\omega^{*}$, existe $\alpha<\omega_{1}$ tal que $\mathfrak{u} \in W_{\alpha}$ e, pela forma como tomamos os $A_{\alpha}$ 's, isto implica em $\bigcap \mathcal{A} \subseteq W_{\alpha}$, o que acarreta $\bigcap \mathcal{A}=\{\mathfrak{u}\}$, pois $\omega^{*}$ é de Hausdorff. Em particular, $\mathcal{A}$ é uma base local para u: se $V$ é uma vizinhança de $\mathfrak{u}$, então existe $\alpha^{\prime}<\omega_{1}$ tal que $\mathfrak{u} \in W_{\alpha^{\prime}} \subseteq V$ e, neste caso, temos $\mathfrak{u} \in A_{\alpha^{\prime}} \subseteq W_{\alpha^{\prime}}$.

Finalmente, se $\left\{V_{n}\right\}_{n \in \omega}$ é uma família de vizinhanças de $\mathfrak{u}$, então para cada $n \in \omega$ existe $\alpha_{n}<\omega_{1}$ tal que $A_{\alpha_{n}} \subseteq V_{n}$, basta então notar que $\alpha=\sup _{n \in \omega} \alpha_{n}<\omega_{1}$ e, desse modo, segue que

$$
\mathfrak{u} \in A_{\alpha} \subseteq \bigcap_{n \in \omega} A_{\alpha_{n}} \subseteq \bigcap_{n \in \omega} V_{n}
$$

provando que $\mathfrak{u}$ é um $P$-ponto.

A densidade do conjunto $\left\{\mathfrak{u} \in \omega^{*}: \mathfrak{u}\right.$ é $P$-ponto $\}$ segue diretamente do item $(d)$ da proposição anterior. 



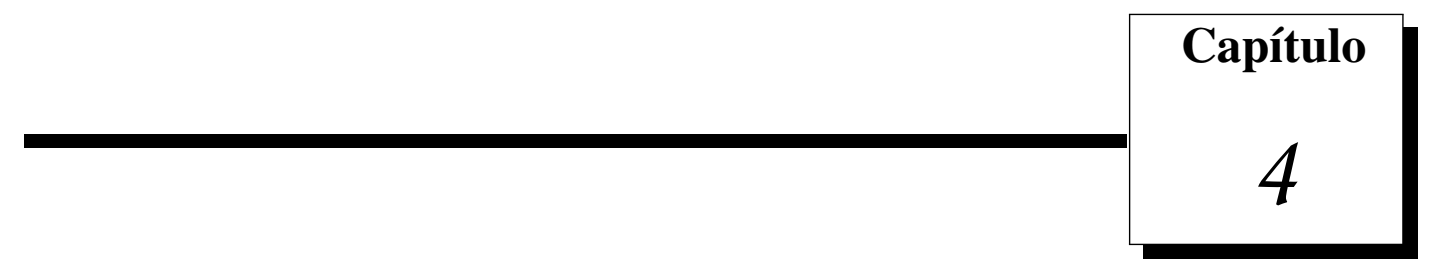

\section{Aplicações: Espaços Produtivamente de}

\section{Lindelöf}

Ao contrário do que ocorre com a compacidade, a propriedade de Lindelöf não é preservada pelo produto em geral. A reta de Sorgenfrey, $\mathbb{R}_{S}$, que consiste do conjunto dos números reais munido da topologia gerada pelos intervalos $[a, b[$, com $a<b$, é um exemplo de espaço de Lindelöf cujo quadrado não é de Lindelöf: a "diagonal" $\left\{(-x, x): x \in \mathbb{R}_{S}\right\}$ é um fechado, discreto e não-enumerável que atesta isso.

Assim, faz sentido considerar a classe dos espaços produtivamente de Lindelöf, isto é, dos espaços cujo produto com qualquer espaço de Lindelöf é de Lindelöf. É natural então nos perguntarmos por caracterizações equivalentes para tal propriedade, preferencialmente por aquelas que dependam unicamente do espaço considerado. Assumindo $\mathrm{CH}$, existe uma caracterização para espaços produtivamente de Lindelöf, mas apenas para espaços cujo peso não exceda $\aleph_{1}$. No entanto, de modo geral, uma caracterização válida para qualquer espaço topológico ainda não é conhecida.

Neste capítulo, abordaremos algumas aplicações de espaços de funções relacionados com o problema acima. Em particular, na última seção introduziremos alguns pequenos cardinais, os quais serão usados em nossa discussão sobre o Problema de Michael. 


\subsection{Espaços de Alster I: Aplicações de $C_{\mathcal{B}}$-teoria}

Para um espaço topológico $X$, uma coleção $\mathcal{U}$ de conjuntos $G_{\delta}$ de $X$ tal que para todo $K \subseteq X$ compacto existe $U \in \mathcal{U}$ com $K \subseteq U$ será chamada de $K_{\delta}$-cobertura. Diremos que $X$ é um espaço de Alster se para toda $K_{\delta}$-cobertura existir uma subcobertura enumerável; se valer que para toda $K_{\delta}$-cobertura existe uma $K_{\delta}$-subcobertura enumerável, diremos que $X$ é um espaço fortemente de Alster.

Em [2], assumindo $\mathrm{CH}, \mathrm{K}$. Alster provou que se $X$ é produtivamente de Lindelöf e $w(X) \leq$ $\aleph_{1}$, então $X$ é de Alster. Em ZFC, temos o seguinte

Teorema 4.1.1 (Alster, [2], Teorema 1). Espaços de Alster são produtivamente de Lindelöf.

Demonstração. Sejam $X$ um espaço de Alster, $Y$ um espaço de Lindelöf e $\mathcal{W}=\left\{W_{i}: i \in I\right\}$ uma cobertura para $X \times Y$, que sem perda generalidade supomos ser fechada por reuniões finitas. Assim, para $K \subseteq X$ compacto não vazio fixado, para cada $y \in Y$ existe $i(K, y) \in I$ tal que $K \times\{y\} \subseteq W_{i(K, y)}$. Agora, pelo Teorema de Wallace, existem abertos $U_{i(K, y)} \subseteq X$ e $V_{i(K, y)} \subseteq Y$ satisfazendo $K \times\{y\} \subseteq U_{i(K, y)} \times V_{i(K, y)} \subseteq W_{i(K, y)}$.

Logo, $\left\{V_{i(K, y)}: y \in Y\right\}$ é uma cobertura por abertos para $Y$, donde segue que existe $Y_{K} \in[Y]^{\leq \omega}$ tal que $Y=\bigcup\left\{V_{i(K, y)}: y \in Y_{K}\right\}$. Notemos então que $G_{K}=\bigcap_{y \in Y_{K}} U_{i(K, y)}$ é um $G_{\delta}$ de $X$ que contém $K$ e, por conseguinte, $\left\{G_{K}: K \subseteq X\right.$ é compacto não vazio $\}$ é uma $K_{\delta}$-cobertura para $X$. Agora, a hipótese sobre $X$ nos permite obter uma família enumerável $\left\{K_{n}: n \in \omega\right\}$ de compactos de $X$ tal que $\left\{G_{K_{n}}: n \in \omega\right\}$ recobre $X$. Claramente, a família $\left\{U_{i\left(K_{n}, y\right)} \times V_{i\left(K_{n}, y\right)}: y \in Y_{K_{n}}, n \in \omega\right\}$ é uma cobertura enumerável para $X \times Y$ que refina $\mathcal{W}$.

Diremos que uma coleção $\mathcal{U}$ de $G_{\delta}$ 's de $X$ é uma $\mathcal{B}_{\delta}$-cobertura para $X$ se todo elemento de $\mathcal{B}$ estiver contido em algum membro de $\mathcal{U}$. No Capítulo 2, vimos que se $\mathcal{B}$ é uma bornologia com base compacta sobre $X$, então com a terminologia que introduzimos aqui, $C_{\mathcal{B}}(X)$ é produtivamente de tightness enumerável se, e somente se, toda $\mathcal{B}_{\delta}$-cobertura para $X$ admite $\mathcal{B}_{\delta}$-subcobertura enumerável. Assim, como em tais casos toda $K_{\delta}$-cobertura é $\mathcal{B}_{\delta}$-cobertura, o seguinte corolário é imediato: 
Corolário 4.1.2. Sejam $X$ um espaço de Tychonoff e $\mathcal{B}$ uma bornologia sobre $X$ com base compacta. Se $\aleph_{0} \in S p\left(C_{\mathcal{B}}(X)\right)$, então $X$ é de Alster e, por conseguinte, é produtivamente de Lindelöf.

Em $C_{p}$-teoria, uma consequência imediata de $X$ ser produtivamente de Lindelöf se dá por meio do Corolário 2.3.3 neste caso, $X$ é de Lindelöf, donde segue que $\sup \left\{l\left(X^{n}\right): n \in \omega\right\}=$ $\aleph_{0}$, donde temos $t\left(C_{p}(X)\right)=\aleph_{0}$. Além disso, quando $X$ é hemicompacto, temos $\chi\left(C_{k}(X)\right)=$ $\aleph_{0}$, e o corolário anterior aliado ao Corolário 2.4 .8 implicam que $X$ é de Alster. Em particular, todo espaço compacto é de Alster e, por conseguinte, também são de Alster todos os espaços $\sigma$-compactos. É então natural nos perguntarmos se vale a recíproca, mais precisamente:

Questão 4.1.1. Em geral, se $X$ é produtivamente de Lindelöf, então existe alguma bornologia $\mathcal{B}$ sobre $X$ com base compacta tal que $\aleph_{0} \in S p\left(C_{\mathcal{B}}(X)\right)$ ? Se não, para quais classes de espaços tal implicação é verdadeira?

Observe que se $X$ é um espaço topológico no qual todo compacto está contido num compacto $G_{\delta}$, então a condição de Alster é equivalente a $\sigma$-compacidade, ao passo que a hemicompacidade coincide com a condição de fortemente Alster. Assim, por exemplo, um espaço métrico é $\sigma$-compacto se, e somente se, é de Alster, pois subespaços fechados de espaços métricos são $G_{\delta}$.

Assim, os espaços $\mathbb{R}$ e $\mathbb{Q}$ são exemplos de espaços produtivamente de Lindelöf que verificam a recíproca do Corolário 4.1.2 para bornologias relativamente "diferentes". Como $\mathbb{R}$ é não-enumerável, temos $l\left(\mathbb{R}_{\delta}\right)=\mathfrak{c}$, donde segue que $\aleph_{0} \notin S p\left(C_{p}(\mathbb{R})\right)$, entretanto, por $\mathbb{R}$ ser hemicompacto, temos $\chi\left(C_{k}(\mathbb{R})\right)=\aleph_{0} \in S p\left(C_{k}(\mathbb{R})\right)$. Por sua vez, temos $\aleph_{0} \in S p\left(C_{p}(\mathbb{Q})\right)$ por $\mathbb{Q}$ ser enumerável, mas $\aleph_{0} \notin S p\left(C_{k}(\mathbb{Q})\right)$ pois $\mathbb{Q}$ não é hemicompacto: considere $\left\{K_{n}: n \in \omega\right\}$ uma família enumerável de compactos de $\mathbb{Q}$ e note que, para cada $n \in \omega,] 0, \frac{1}{n+1}\left[\cap \mathbb{Q} \nsubseteq K_{n}\right.$, caso contrário existiria uma sequência em $K_{n}$ convergindo para um número irracional, contrariando a compacidade de $K_{n}$; logo, para cada $n \in \omega$ existe $\left.x_{n} \in\right] 0, \frac{1}{n+1}\left[\cap \mathbb{Q} \backslash K_{n}\right.$, donde segue que $K=\left\{x_{n}: n \in \omega\right\} \cup\{0\}$ é um compacto que satisfaz $K \nsubseteq K_{n}$ para todo $n \in \omega$.

Já vimos na Seção 1.4 que o espaço $\omega^{\omega}$ não é um espaço $\sigma$-compacto. Como tal espaço é homeomorfo a $\mathbb{P}$, o espaço dos números irracionais com a topologia de subespaço herdada de $\mathbb{R}$ ( $c f$. Exercício $24 \mathrm{~K}$ de [50]), segue que $\mathbb{P}$ não é de Alster. Isso nos permite concluir que 
$C_{\mathcal{B}}(\mathbb{P})$ não é produtivamente de tightness enumerável, para qualquer bornologia $\mathcal{B}$ em $\mathbb{P}$ com base compacta - em verdade, por $\mathbb{P}$ ser normal, segue que $\aleph_{0} \notin S p\left(C_{\mathcal{B}}(\mathbb{P})\right)$ para qualquer bornologia $\mathcal{B}$ em $X$. Assim, se a resposta para a Questão 4.1.1 for afirmativa, seguirá em particular que $\mathbb{P}$ não é produtivamente de Lindelöf, o que ainda não é conhecido em ZFC.

Em [5], Aurichi e Bella provaram a seguinte cadeia de implicações, para qualquer espaço de Tychonoff $X$ :

$$
\mathrm{II} \uparrow \mathrm{G}_{1}\left(\Omega_{x}, \Omega_{x}\right) \Rightarrow \aleph_{0} \in S p(x, X) \Rightarrow \mathrm{S}_{1}\left(\Omega_{x}, \Omega_{x}\right),
$$

onde $\Omega_{x}=\{A \subset X: x \in \bar{A} \backslash A\}$ e $x \in X$. Isso nos permite "conectar" os Teoremas 2.3.6 2.3.13 e 2.3.23 com as observações feitas nesta seção: se $\mathcal{B}$ é uma bornologia com base compacta em $X$, temos

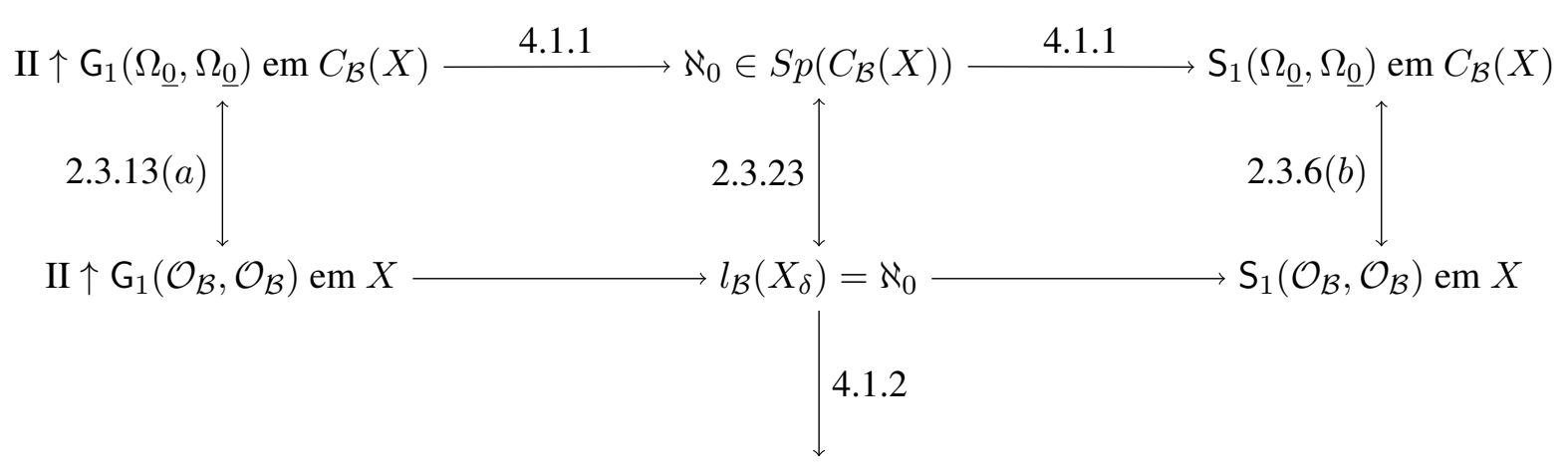

$X$ é de Alster

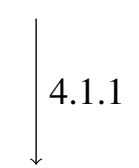

$X$ é produtivamente de Lindelöf 


\subsection{Espaços de Alster II: Aplicações da compactificação de Stone- Čech}

Nesta seção, apresentaremos uma condição suficiente para que um espaço de Tychonoff não seja produtivamente de Lindelöf, seguindo essencialmente as referências [1, 12]. Para motivar as definições que daremos, considere um espaço de Tychonoff $(X, \tau)$, e suponha que exista $Y \subseteq X$ com a seguinte propriedade:

existe uma topologia $\sigma$ em $Y$ tal que $\sigma \supseteq \tau \uparrow Y$ e $(Y, \sigma)$ não é de Lindelöf,

onde $\tau \uparrow Y:=\{U \cap Y: U \in \tau\}$.

Como isso é trivialmente válido se $(X, \tau)$ não for de Lindelöf, vamos supor também que $X$ é de Lindelöf. Se conseguirmos "mergulhar" $(Y, \sigma)$ como um subespaço (não-fechado) num espaço de Lindelöf $\mathcal{L}$ tal que $\mathcal{D}:=\{(y, y): y \in Y\}$ seja fechado em $(X, \tau) \times \mathcal{L}$, então seguirá que $X$ não é produtivamente de Lindelöf: como $(Y, \sigma)$ não é de Lindelöf, existe uma cobertura $\mathcal{U}$ para $Y$ por $\sigma$-abertos que não admite subcobertura enumerável, e assim $\{X \times U: U \in \mathcal{U}\}$ é uma cobertura para $\mathcal{D}$ que não admite subcobertura enumerável, e o resultado segue por $\mathcal{D}$ ser fechado em $(X, \tau) \times \mathcal{L}$.

Como obter o espaço $\mathcal{L}$ ? Por $(X, \tau)$ ser de Tychonoff, podemos considerar $\tau=\rho \uparrow X$, onde $\rho$ é uma topologia compacta de Hausdorff sobre um conjunto $L$, tal que $(L, \rho)=\beta X$ e, sem perda de generalidade, podemos supor que $X$ está (propriamente) contido em $L$. Seja $\mathcal{L}=X^{*} \cup Y$, onde $X^{*}=\beta X \backslash X$, e considere sobre $\mathcal{L}$ a topologia $\mathcal{T}$ definida da seguinte forma: $A \subseteq \mathcal{L}$ é $\mathcal{T}$-aberto se, e somente se, para todo $a \in A$ existe $U \in \sigma$ tal que $a \in U \subseteq A$ se $a \in Y$, ou existe $V \in \rho$ tal que $a \in V$ e $(V \cap Y) \cup(V \backslash X) \subseteq A$ caso $a \in X^{*}$, como ilustrado na Figura 4.1 .

Claramente $(\mathcal{L}, \mathcal{T})$ é um espaço de Hausdorff que satisfaz parte das condições impostas: contém $(Y, \sigma)$ como subespaço não necessariamente fechado e $\mathcal{D}$ é fechado em $(X, \tau) \times \mathcal{L}$. Além disso, note que $\left(X^{*}, \rho\right)$ é um subespaço fechado de $(\mathcal{L}, \mathcal{T})-$ se $a \notin X^{*}$, então $a \in Y$ e assim existe $U \in \sigma$ tal que $a \in U \subseteq Y$. Logo, quando o espaço $(\mathcal{L}, \mathcal{T})$ é de Lindelöf, necessariamente temos $X^{*}$ de Lindelöf. Note ainda que se $\mathcal{U}$ é uma cobertura para $X^{*}$ por 


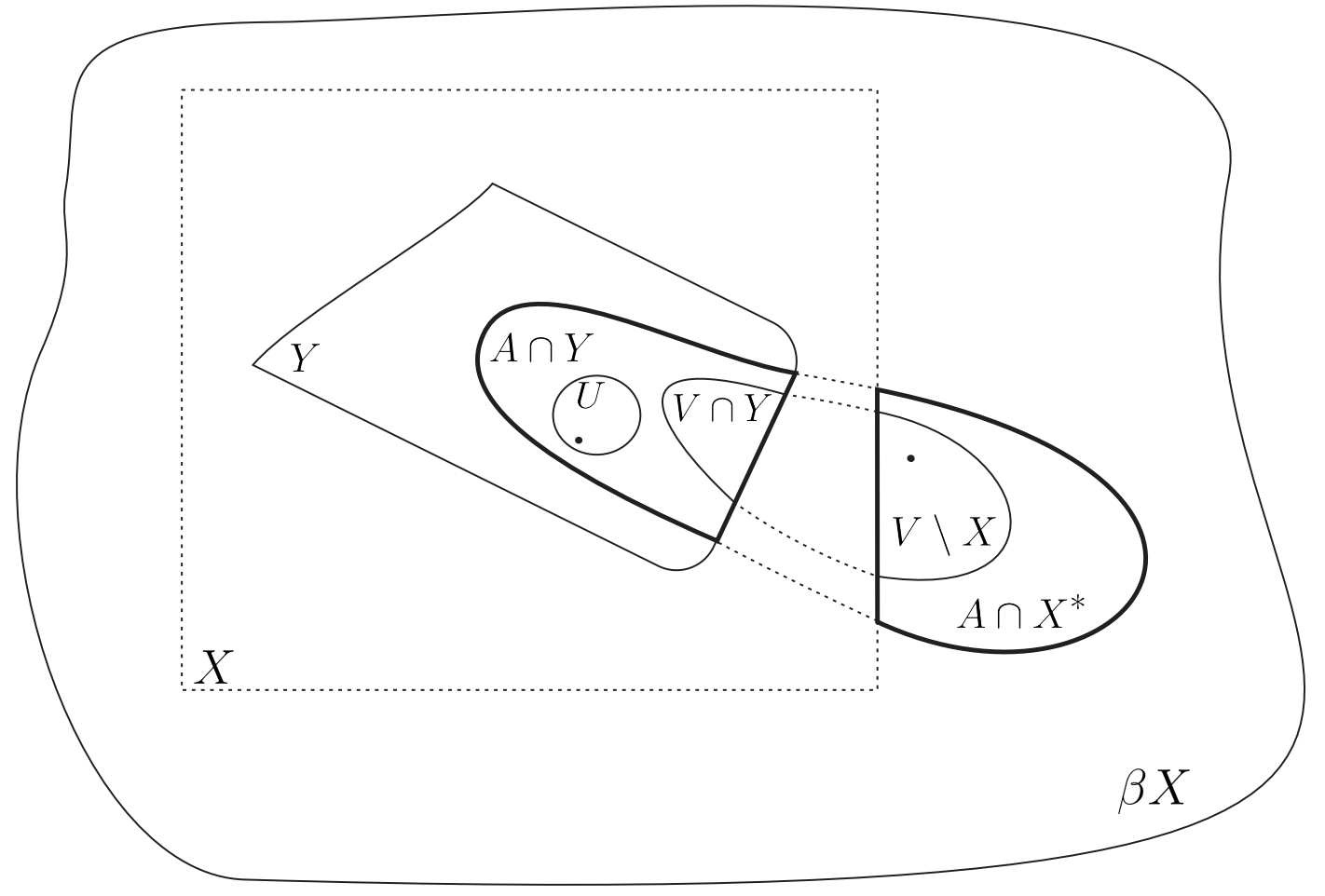

Figura 4.1: Um aberto em $(\mathcal{L}, \mathcal{T})$.

abertos de $\beta X$, então $\beta X \backslash \bigcup \mathcal{U} \subseteq X$ é fechado em $\beta X$ e, por conseguinte, é um subespaço compacto de $(X, \tau)$. Em vista disso, a seguinte condição que impomos sobre $Y$ é bastante natural:

se $K \subset X$ é compacto na topologia $\tau$, então $K \cap Y$ é de Lindelöf na topologia $\sigma$.

Assim, se $\mathcal{U}$ é uma cobertura aberta para $\mathcal{L}$, podemos considerar uma subcoleção $\mathcal{U}^{\prime} \subseteq \mathcal{U}$ de abertos (possivelmente refinando os originais, e obtendo restrições de abertos de $\beta X$ ) tais que $X^{*} \subseteq \cup \mathcal{U}^{\prime}$, donde segue que $K=\left(\beta X \backslash \cup \mathcal{U}^{\prime}\right) \cap Y$ é um espaço de Lindelöf em $(Y, \sigma)$ e, por conseguinte, nos dá $\mathcal{U}^{\prime \prime} \subseteq \mathcal{U}$ uma subcoleção enumerável tal que $K \subseteq \cup \mathcal{U}^{\prime \prime}$. Note então que, se pudéssemos obter $\mathcal{U}^{\prime}$ enumerável desde o princípio, então $\mathcal{V}=\mathcal{U}^{\prime} \cup \mathcal{U}^{\prime \prime}$ seria uma subcobertura enumerável de $\mathcal{U}$ para $\mathcal{L}$; em particular, isso mostra que se $X^{*}$ é de Lindelöf, então $(\mathcal{L}, \mathcal{T})$ é de Lindelöf.

Um espaço de Tychonoff $X$ tal que $X^{*}$ é de Lindelöf é chamado de espaço de Lindelöf no infinito. Assim, a argumentação apresentada acima "quase" demonstra o seguinte teorema: 
Teorema 4.2.1 (Alas et al.,[1], Teorema 2.4; Tall et al., [12], Teorema 2). Seja X um espaço regular, de Lindelöf e de Lindelöf no infinito. Se existir $Y \subseteq X$ satisfazendo as condições (4.2.1) e (4.2.2) acima, então existe um espaço de Lindelöf regular $\mathcal{L}$ tal que $X \times \mathcal{L}$ não é de Lindelöf.

De fato, nossa discussão anterior não garante a regularidade de $\mathcal{L}$. Contudo, uma vez conhecido tal espaço $\mathcal{L}$, podemos obter um espaço regular que satisfaça as condições do teorema. Para ver isso, faremos algumas observações preliminares. Considere $X$ e $Y$ espaços topológicos e $\varphi: X \rightarrow \wp(Y)$ uma função com as seguintes propriedades:

(a) $\varphi(x) \subseteq Y$ é compacto para todo $x \in X$ (dizemos que $\varphi$ é valorada em compactos);

(b) se $V \subseteq Y$ é aberto, então $\varphi^{-1}(V):=\{x \in X: \varphi(x) \subseteq V\}$ é aberto em $X$ (dizemos que $\varphi$ é semicontínua superiormente).

Em tais condições, $\bigcup \varphi[X]$ é um espaço de Lindelöf sempre que $X$ é de Lindelöf: de fato, se $\mathcal{U}$ é uma cobertura aberta para $\bigcup \varphi[X]$ fechada por reuniões finitas, então $\left\{\varphi^{-1}(U): U \in \mathcal{U}\right\}$ é uma cobertura para $X$ e, por conseguinte, existe $\left\{U_{n}: n \in \omega\right\} \subseteq \mathcal{U}$ tal que $\left\{\varphi^{-1}\left(U_{n}\right): n \in \omega\right\}$ recobre $X$; é fácil ver que $\left\{U_{n}: n \in \omega\right\}$ recobre $\bigcup \varphi[X]$.

Agora, para um conjunto $\mathscr{A}$ não vazio qualquer, a bijeção $\chi: \wp(\mathscr{A}) \rightarrow 2^{\mathscr{A}}$, que a cada $A \subseteq \mathscr{A}$ associa a função característica $\chi_{A}$, nos permite ver $\wp(\mathscr{A})$ como um espaço topológico compacto, zero-dimensional e $T_{2}$. Em particular, note que pela correspondência $\chi$, os abertos básicos de $\wp(\mathscr{A})$ são conjuntos da forma

$$
\mathcal{A}(F, G):=\{A \in \wp(\mathscr{A}): F \subseteq A \subseteq \mathscr{A} \backslash G\}
$$

para $F, G \in[\mathscr{A}]^{<\omega}$, embora também seja útil observar que para $A \in \wp(\mathscr{A})$, as vizinhanças básicas de $A$ são da forma

$$
\mathcal{V}(A, F):=\{B \in \wp(\mathscr{A}): B \cap F=A \cap F\},
$$

$\operatorname{para} F \in[\mathscr{A}]^{<\omega}$. 
Considere então $\mathcal{U}=\left\{U_{i}: i \in I\right\}$ uma cobertura por abertos para algum espaço topológico $X$. A função $\Phi_{X, \mathcal{U}}: X \rightarrow \wp(\wp(I))$, definida por

$$
\Phi_{X, \mathcal{U}}(x):=\left\{A \subseteq I:\left\{i \in I: x \in U_{i}\right\} \subseteq A\right\}
$$

satisfaz as condições (a) e (b) acima.

Para a condição (a), basta notar que $\Phi_{X, \mathcal{U}}(x)$ é fechado em $\wp(I)$, que é compacto. Para verificar (b), considere $\mathcal{A} \subseteq \wp(I)$ aberto, $x \in \Phi_{X, \mathcal{U}}^{-1}(\mathcal{A})$ e, para cada $B \in \Phi_{X, \mathcal{U}}(x)$ tome $F_{B} \in[I]^{<\omega}$ tal que $B \in \mathcal{V}\left(B, F_{B}\right) \subseteq \mathcal{A}$. Como $I_{x}:=\left\{i \in I: x \in U_{i}\right\} \subseteq B$, podemos supor que $i_{0} \in F_{B}$ para algum $i_{0} \in I_{x}$ fixado, para todo $B \in \Phi_{X, \mathcal{U}}(x)$. Pela compacidade de $\Phi_{X, \mathcal{U}}(x)$, existem $B_{0}, \ldots, B_{n} \in \Phi_{X, \mathcal{U}}(x)$ tais que $\Phi_{X, \mathcal{U}}(x) \subseteq \bigcup_{i \leq n} \mathcal{V}\left(B_{i}, F_{B_{i}}\right) \subseteq \mathcal{A}$; pela hipótese sobre os $F_{B}$ 's, temos $F=\left(\bigcup_{i \leq n} F_{B_{i}}\right) \cap I_{x} \neq \emptyset$. Afirmamos que $U=\bigcap_{i \in F} U_{i}$ é um aberto de $X$ que atesta $x \in U \subseteq \Phi_{X, \mathcal{U}}^{-1}(\mathcal{A})$. De fato, para $y \in U$, temos $\Phi_{X, \mathcal{U}}(y) \subseteq \mathcal{A}$ : ora, por $y \in U$ temos $F \subseteq I_{y} \subseteq C$ para todo $C \in \Phi_{X, \mathcal{U}}(y)$, donde segue que $C \cup I_{x} \in \Phi_{X, \mathcal{U}}(x)$ e, consequentemente, existe $i \leq n$ tal que $C \cup I_{x} \in \mathcal{V}\left(B_{i}, F_{B_{i}}\right) \subseteq \mathcal{A}$; logo, $C \in \mathcal{V}\left(B_{i}, F_{B_{i}}\right)$, como queríamos.

Em suma, provamos o seguinte

Lema 4.2.2 (Adaptado de Zdomsky, [51], Lema 2). Sejam X e Y espaços topológicos e uma função $\Phi: X \rightarrow \wp(Y)$ valorada em compactos e semicontínua superiormente.

(a) Se X é de Lindelöf, então $\varphi[X]$ é de Lindelöf.

(b) $\operatorname{Se} \mathcal{U}=\left\{U_{i}: i \in I\right\}$ é uma cobertura aberta para $X$, então $\Phi_{X, \mathcal{U}}$ como definida em (4.2.5) é uma função valorada em compactos e semicontínua superiormente.

Proposição 4.2.3 (Tall et al., [12], Lema 1). Seja X um espaço de Lindelöf. Se existe um espaço de Lindelöf $Z$ tal que $X \times Z$ não é de Lindelöf, então existe um espaço de Lindelöf regular $Z^{\prime}$ tal que $X \times Z^{\prime}$ não é de Lindelöf.

Demonstração. Seja $\mathcal{U}=\left\{U_{\alpha} \times V_{\alpha}: \alpha<\kappa\right\}$ uma cobertura por abertos básicos para $X \times Z$ que não admite subcobertura enumerável. Sejam $\mathcal{U}_{0}=\left\{U_{\alpha}: \alpha<\kappa\right\}, \mathcal{U}_{1}=\left\{V_{\alpha}: \alpha<\kappa\right\}$ e considere as funções $\Phi_{X, \mathcal{U}_{0}}$ e $\Phi_{Z, \mathcal{U}_{1}}$ como em 4.2.5). Pelo Lema anterior, segue que $X^{\prime}=$ $\bigcup \Phi_{X, \mathcal{U}_{0}}[X]$ e $Z^{\prime}=\bigcup \Phi_{Z, \mathcal{U}_{1}}[Z]$ são subespaços de Lindelöf de $\wp(\kappa)$, em particular, note que $Z^{\prime}$ é regular. 
Agora, note que se $X \times Z^{\prime}$ é de Lindelöf, então $X^{\prime} \times Z^{\prime}$ é de Lindelöf: para uma cobertura $\mathcal{V}$ para $X^{\prime} \times Z^{\prime}$ fechada por reuniões finitas, use o fato de que $\Phi_{X, \mathcal{U}_{0}}$ é valorada em compactos para obter um refinamento $\mathcal{V}^{\prime}$ por abertos básicos (como no Teorema 4.1.1) e, em seguida, use o fato de que $\Phi_{X, \mathcal{U}_{0}}$ é semicontínua superiormente para induzir uma cobertura apropriada em $X \times Z^{\prime}$, donde o resultado segue facilmente.

Assim, é suficiente provar que $X^{\prime} \times Z^{\prime}$ não é de Lindelöf para concluir a demonstração. Para ver isso, chame $T_{\alpha}=\{D \subseteq \kappa: \alpha \in D\}$ para todo $\alpha<\kappa$ e considere $\mathcal{W}=\left\{W_{\alpha}: \alpha<\kappa\right\}$, onde $W_{\alpha}=T_{\alpha} \times T_{\alpha}$. Note que $\mathcal{W}$ é uma cobertura para $X^{\prime} \times Z^{\prime}$ e, para qualquer $I \subseteq \kappa$, $\left\{U_{\alpha} \times V_{\alpha}: \alpha \in I\right\}$ é uma cobertura para $X \times Z$ sempre que $\left\{W_{\alpha}: \alpha \in I\right\}$ cobre $X^{\prime} \times Z^{\prime}$, donde segue que $\mathcal{W}$ não admite subcobertura enumerável.

Observação 4.2.4. O Teorema 4.2.1 foi primeiramente provado em [1], onde condições mais restritivas do que (4.2.1) e (4.2.2) são impostas sobre $Y$ a fim de garantir a regularidade do espaço $\mathcal{L}$ obtido; em [12], os autores observam que tais condições podem ser "relaxadas" para (4.2.1) e 4.2.2), tendo em vista a Proposição anterior.

Corolário 4.2.5 (Alas et al., [1], Corolário 2.5). Seja X um espaço de Lindelöf regular e de Lindelöf no infinito. Se existe $Y \subseteq X$ não-enumerável tal que $K \cap Y$ é enumerável para todo $K \subseteq X$ compacto, então $X$ não é produtivamente de Lindelöf.

Demonstração. Considere sobre $Y$ a topologia discreta e aplique o Teorema 4.2.1.

Diremos que $X$ é um espaço de tipo contável se para todo $K \subseteq X$ compacto existem um subespaço compacto de $K^{\prime} \supseteq K$ e uma base local enumerável em $K^{\prime}$ ( $c f$. definição de espaço ponto-contável, na Subseção 2.2.1). Quando $X$ é de Tychonoff, tal classe de espaços coincide exatamente com os espaços de Lindelöf no infinito ${ }^{1}$. Observe que todo espaço com peso enumerável é de tipo contável.

Corolário 4.2.6. Se X é um espaço de Lindelöf, não-enumerável, que satisfaz o primeiro axioma de enumerabilidade e no qual todo subespaço compacto é enumerável, então X não é produtivamente de Lindelöf.

\footnotetext{
${ }^{1}$ Resultado devido a Henriksen e Isbell [20].
} 
Demonstração. Basta notar que, neste caso, todo compacto tem base local enumerável, e assim $X$ é de Lindelöf no infinito, de modo que o resultado segue pelo corolário anterior.

Para um espaço topológico $X$, definimos o grau de Alster de $X$, denotado por $\mathscr{A}(X)$, como sendo o menor cardinal $\kappa$ tal que para toda $K_{\delta}$-cobertura de $X$ existe uma subcobertura de cardinalidade $\leq \kappa$. Por sua vez, $\mathscr{K}(X)$ é o menor cardinal da forma $|\mathcal{K}|$, onde $\mathcal{K}$ é uma cobertura para $X$ por compactos. Em geral, $\mathscr{A}(X) \leq \mathscr{K}(X)$ : se $\mathcal{U}$ é uma $K_{\delta}$-cobertura para $X$ e $\mathcal{V}$ é uma cobertura por compactos para $X$, basta tomar $U_{K} \in \mathcal{U}$ para cada $K \in \mathcal{V}$ tal que $K \subseteq U_{K}$ e notar que $\left\{U_{K}: K \in \mathcal{V}\right\}$ é uma cobertura por $G_{\delta}$ 's para $X$. Veremos que para espaços de tipo contável, vale $\mathscr{A}(X)=\mathscr{K}(X)$.

Proposição 4.2.7 (Alas et al., [1], Proposição 4.13). Para um espaço de tipo contável $X$ vale que $\mathscr{A}(X)=\mathscr{K}(X)$. Em particular, neste caso $X$ é de Alster se, e somente se, $X$ é $\sigma$ compacto.

Demonstração. Já temos $\mathscr{A}(X) \leq \mathscr{K}(X)$, vamos provar que $\mathscr{K}(X) \leq \mathscr{A}(X)$. Seja $\mathcal{K}$ a coleção de todos os compactos de $X$, e para cada $K \in \mathcal{K}$ tome um $G_{\delta}$ da forma $\bigcap \mathfrak{B}_{K}$, onde $\mathfrak{B}_{K}$ é uma base local em algum compacto $K^{\prime}$ que contém $K$. Logo, $\left\{\cap \mathfrak{B}_{K}: K \in \mathcal{K}\right\}$ é uma $K_{\delta}$-cobertura para $X$, donde segue que existe $\left\{\cap \mathfrak{B}_{K_{\alpha}}: \alpha<\mathscr{A}(X)\right\}$ uma subcobertura e, consequentemente, $X=\bigcup_{\alpha<\mathscr{A}(X)} K_{\alpha}^{\prime}$.

Corolário 4.2.8. Se $X$ é de Lindelöf no infinito e $\mathscr{A}(X)=\aleph_{1}$, então $X$ não é produtivamente de Lindelöf.

Demonstração. Seja $\mathcal{U}=\left\{U_{\alpha}: \alpha<\omega_{1}\right\}$ uma $K_{\delta}$-cobertura que não admite subcobertura enumerável. Chame $U_{0}=U_{i_{0}}$ e tome $x_{0} \in U_{i_{0}}$; agora, para $\alpha<\omega_{1}$, suponha escolhidos $\left(x_{\beta}\right)_{\beta<\alpha}$ e $\left(U_{i_{\beta}}\right)_{\beta<\alpha}$ tais que, para cada $\beta<\alpha, i_{\beta}=\min \left\{U_{\gamma}: U_{\gamma} \backslash \bigcup_{\xi<\beta} U_{i_{\xi}} \neq \emptyset\right\}, U_{i_{\beta}} \in \mathcal{U}$ e $x_{\beta} \in U_{i_{\beta}} \backslash \bigcup_{\xi<\beta} U_{i_{\xi}}$. Note que $A_{\alpha}:=\left\{U_{\gamma}: U_{\gamma} \backslash \bigcup_{\beta<\alpha} U_{i_{\beta}} \neq \emptyset\right\} \neq \emptyset$, pois $\mathcal{U}$ não admite subcobertura enumerável e $\alpha<\omega_{1}$ é enumerável, chame então $i_{\alpha}=\min A_{\alpha}$. Assim, existem $\left\{U_{i_{\alpha}}: \alpha<\omega_{1}\right\} \subseteq \mathcal{U}$ e $\left(x_{\alpha}\right)_{\alpha<\omega_{1}}$ tais que $x_{\alpha} \in U_{i_{\alpha}} \backslash \bigcup_{\beta<\alpha} U_{i_{\beta}}$ e $i_{\alpha} \geq \alpha$ para todo $\alpha<\omega_{1}$. Finalmente, se $K \subseteq X$ é compacto, então $K \subseteq U_{\alpha}$ para algum $\alpha<\omega_{1}$, e como $i_{\alpha} \geq \alpha$, temos $K \cap\left\{x_{\xi}: \xi<\omega_{1}\right\} \subseteq\left\{x_{\xi}: \xi<\alpha\right\}$, acarretando que $K \cap\left\{x_{\alpha}: \alpha<\omega_{1}\right\}$ é enumerável. Logo, o resultado segue pelo Corolário 4.2.5. 
Em particular, segue que se $X$ é de Lindelöf no infinito e não é $\sigma$-compacto, então $X$ não é produtivamente de Lindelöf.

\subsection{Pequenos Cardinais e o Problema de Michael}

Um pequeno cardinal é, grosso modo, qualquer cardinal $\kappa$ com $\aleph_{1} \leq \kappa \leq \mathfrak{c}$, tal que $\kappa$ é definido, de alguma forma, em termos de $\omega$; os cardinais que apresentaremos são definidos em termos do espaço $\omega^{\omega}$, da seguinte maneira: para uma condição $\mathcal{P}$ sobre os subconjuntos de $\omega^{\omega}$, $\kappa:=\min \left\{|A|: A \subseteq \omega^{\omega}\right.$ satisfaz $\left.\mathcal{P}\right\}$. Como $\omega^{\omega}$ é um espaço de funções, o estudo de tais pequenos cardinais se qualifica como tópico deste trabalho per se.

Definimos uma relação $\leq^{*}$ sobre $\omega^{\omega}$ declarando

$$
f \leq^{*} g \text { se, e somente se, }|\{n \in \omega: f(n)>g(n)\}|<\aleph_{0}
$$

que é claramente uma relação reflexiva $\left(f \leq^{*} f\right)$ e transitiva $\left(f \leq^{*} g\right.$ e $\left.g \leq^{*} h \Rightarrow f \leq^{*} h\right)$, mas não é uma relação assimétrica (pode ocorrer $f \leq^{*} g$ e $g \leq^{*} f$ sem que $f=g$ ), e tampouco é uma relação total, no sentindo de que quaisquer duas funções $f, g \in \omega^{\omega}$ estejam relacionadas: chamando $P=\{2 n: n \in \omega\}$, temos tanto $\chi_{P} \mathbb{Z}^{*} \chi_{\omega \backslash P}$ quanto $\chi_{\omega \backslash P} \mathbb{Z}^{*} \chi_{P}$, onde $\chi_{P}$ e $\chi_{\omega \backslash P}$ são as funções características de $P$ e $\omega \backslash P$, respectivamente. Análoga à relação $\leq^{*}$, declaramos $f<^{*} g$ se, e somente se, $|\{n \in \omega: f(n) \geq g(n)\}|<\aleph_{0}$.

Dizemos que $D \subseteq \omega^{\omega}$ é uma família dominante (dominating family) se $D$ for cofinal em $\left(\omega^{\omega}, \leq^{*}\right)$, ou seja, se para toda $f \in \omega^{\omega}$ existe $g \in D$ tal que $f \leq^{*} g$. O número de dominância (dominating number), denotado por $\mathfrak{d}$, é o menor cardinal da forma $|D|$, onde $D$ é uma família dominante em $\omega^{\omega}$, mais formalmente:

$$
\mathfrak{d}:=\min \left\{|D|: D \subseteq \omega^{\omega} \text { é dominante }\right\}
$$

Claramente temos $\mathfrak{d} \leq \mathfrak{c}$, já que $\omega^{\omega}$ é trivialmente dominante.

Dizemos que $B \subseteq \omega^{\omega}$ é uma família ilimitada (unbounded family) se $B$ é ilimitada em $\left(\omega^{\omega}, \leq^{*}\right)$, i.e., se não existe $f \in \omega^{\omega}$ que limite todas as funções de $B$ com respeito a $\leq^{*}$ ou, ainda mais precisamente, para toda $f \in \omega^{\omega}$ existe $g \in B$ tal que $g \not^{*} f$. Definimos o número 
de ilimitância (unbounding number), denotado por $\mathfrak{b}$, como sendo o menor cardinal da forma $|B|$, onde $B$ é uma família ilimitada em $\omega^{\omega}$, isto é:

$$
\mathfrak{b}:=\min \left\{|B|: B \subseteq \omega^{\omega} \text { é ilimitada }\right\}
$$

Como toda família dominante é ilimitada, segue que $\mathfrak{b} \leq \mathfrak{d} \leq \mathfrak{c}$. Além disso, note que se $A \subset \omega^{\omega}$ é enumerável, então $A$ não é ilimitada: fazendo $A=\left\{f_{n}: n \in \omega\right\}$, basta notar que $f: \omega \rightarrow \omega$ dada por $f(k)=1+\sum_{i<k} f_{i}(k)$ é tal que $f_{n} \leq^{*} f$ para todo $n \in \omega$. Desse modo, as seguintes desigualdades são válidas em ZFC:

$$
\aleph_{1} \leq \mathfrak{b} \leq \mathfrak{d} \leq \mathfrak{c}
$$

Lembremo-nos de que se $X$ é um espaço topológico, dizemos que $A \subseteq X$ é nunca denso (nowhere dense) se $\operatorname{int}(\bar{A})=\emptyset$, ao passo que $A$ é um conjunto magro se for reunião enumerável de conjuntos nunca densos. Finalmente, $X$ é um espaço de Baire se satisfizer uma (logo, todas) das três condições equivalentes abaixo:

(a) todo conjunto magro em $X$ tem interior vazio;

(b) toda interseção enumerável de abertos densos é densa;

(c) $X$ não é reunião enumerável de fechados nunca densos.

Espaços métricos completos são exemplos de espaços de Baire e, mais geralmente, todo $G_{\delta}$ de um espaço de Baire é de Baire. Como $\mathbb{R}$ é completamente metrizável e $\omega^{\omega}=\mathbb{P}$ é um $G_{\delta}$ de $\mathbb{R}$, segue que $\omega^{\omega}$ é um espaço de Baire.

Chamemos por $\mathcal{M}$ a família dos subconjuntos magros de $\omega^{\omega}$; como a reunião enumerável de conjuntos magros é um conjunto magro e $\omega^{\omega}$ é de Baire, segue que $\omega^{\omega}$ não pode ser reunião enumerável de conjuntos magros. Por outro lado, cada $f \in \omega^{\omega}$ é tal que $\{f\}$ é um conjunto magro, e $\omega^{\omega}=\bigcup_{f \in \omega^{\omega}}\{f\}$. Isso motiva a definição do próximo pequeno cardinal, o número de cobertura (covering number), denotado por $\operatorname{cov}(\mathcal{M})$, o menor cardinal da forma $|C|$, onde $C \subseteq \mathcal{M}$ é tal que $\bigcup C=\omega^{\omega}$, isto é,

$$
\operatorname{cov}(\mathcal{M}):=\min \left\{|C|: C \subseteq \mathcal{M} \wedge \bigcup C=\omega^{\omega}\right\}
$$


e, pelo que discutimos acima, temos $\aleph_{1} \leq \operatorname{cov}(\mathcal{M}) \leq \mathfrak{c}$. Equivalentemente,

$$
\operatorname{cov}(\mathcal{M})=\min \left\{|\mathscr{F}|: \mathscr{F} \subseteq \omega^{\omega} \wedge \forall g \in \omega^{\omega}\left(\exists f \in \mathscr{F}\left(|\{n \in \omega: f(n)=g(n)\}|<\aleph_{0}\right)\right)\right\}
$$

(Bartoszyńsky e Judah, apud Halbeisen [19]), e disso segue que $\operatorname{cov}(\mathcal{M}) \leq \mathfrak{d}$, pois, se $D$ é uma família dominante, então para $g \in \omega^{\omega}$ qualquer existe $f \in D$ tal que $g^{\prime} \leq^{*} f$, onde $g^{\prime}(n)=g(n)+1$ para todo $n$, o que nos dá $|\{n \in \omega: f(n)=g(n)\}|<\aleph_{0}$.

Claramente, $\mathrm{CH}$ acarreta $\aleph_{1}=\mathfrak{b}=\mathfrak{d}=\operatorname{cov}(\mathcal{M})=\mathfrak{c}$, mas outras desigualdades também são consistentes com ZFC $+\neg \mathrm{CH}$, como por exemplo, $\mathfrak{b}<\mathfrak{d}=\operatorname{cov}(\mathcal{M})=\mathfrak{c}(c f$. Halbeisen, [19]). Nesta seção estudaremos algumas influências dos cardinais $\mathfrak{b}, \mathfrak{d}$ e $\operatorname{cov}(\mathcal{M})$ sobre o espaço dos irracionais $\omega^{\omega}$, no que concerne a "Lindelöfcidade" no produto. Ao leitor interessado em mais propriedades dos pequenos cardinais supracitados, bem como em propriedades de outros cardinais que não apresentamos, sugerimos as referências [7, 13, 19, 42].

E. Michael provou que se vale $\mathrm{CH}$, então existe um espaço de Lindelöf regular tal que $X \times \mathbb{P}$ não é de Lindelöf [32]. Saber se tal espaço pode ser construído sem axiomas adicionais (como $\mathrm{CH}$ ) é o que chamamos de Problema de Michael, e um espaço com tais propriedades (de Lindelöf, regular e cujo produto cartesiano com $\mathbb{P}$ resulta num espaço não Lindelöf) é chamado de espaço de Michael. Hoje já se sabe que não precisamos da "força total" de $\mathrm{CH}$ para a obtenção de um espaço de Michael.

De fato, em [34], Moore mostra, entre outras coisas, que existe um espaço de Michael quando assumimos $\mathfrak{d}=\operatorname{cov}(\mathcal{M})$. Por sua vez, na última seção de [42], da Silva apresenta uma prova detalhada de que $\mathfrak{b}=\omega_{1}$ implica a existência de um espaço de Michael: considera-se o conjunto $\mathbb{R}$ munido de uma topologia $\sigma$ mais fina que a usual, na qual os pontos de $\mathbb{P}$ são isolados e, por meio da asserção $\mathfrak{b}=\omega_{1}$, obtém-se um subconjunto não-enumerável $X \subseteq \mathbb{P}$ tal que $Y=X \cup \mathbb{Q}$ visto como subespaço de $(\mathbb{R}, \sigma)$ é de Lindelöf e a "diagonal" $\{(x, x): x \in X\}$ é um subespaço fechado, discreto e não-enumerável de $Y \times \mathbb{P}$.

Vamos seguir a mesma abordagem apresentada em [1] e, por meio do Teorema 4.2.1 demonstrado na seção anterior, provaremos a existência de espaços de Michael para algumas hipóteses adicionais sobre pequenos cardinais. Tal método tem a vantagem de sintetizar di- 
versas construções de espaços de Michael por meio de uma única terminologia técnica, que introduzimos a seguir.

Para um subconjunto $\mathscr{F} \subseteq \omega^{\omega}$, vamos chamar de $\mathscr{F}$-topologia a menor (no sentido da inclusão) topologia em $\omega^{\omega}$ que contém sua topologia usual e tal que os conjuntos da forma $K_{f}:=\left\{g \in \omega^{\omega}: g \leq f\right\}$ sejam abertos, para cada $f \in \mathscr{F}$. Como lidaremos com duas topologias simultaneamente em $\omega^{\omega}$, explicitamos a seguinte convenção ${ }^{2}$; dada uma propriedade topológica $\mathcal{P}$ e $A \subseteq X$, diremos que $A$ é $u$-P (respectivamente, $\mathscr{F}-\mathcal{P}$ ) se $A$ tem a propriedade $\mathcal{P}$ quando visto como subespaço de $\omega^{\omega}$ munido de sua topologia usual (respectivamente, se $A$ tem a propriedade $\mathcal{P}$ quando visto como subespaço de $\omega^{\omega}$ munido da $\mathscr{F}$-topologia).

Dizemos que $E \subseteq \omega^{\omega}$ é uma escala em $\omega^{\omega}$ se $E$ for dominante e $\left(E,<^{*}\right)$ for uma boa ordem; mais precisamente, $E=\left\{f_{\alpha}: \alpha<\gamma\right\}$ é uma escala se $E$ for dominante e valer $f_{\alpha}<^{*} f_{\beta}$ se, e só se, $\alpha<\beta$.

Lema 4.3.1. Existe uma escala em $\omega^{\omega}$ se, e somente se, $\mathfrak{b}=\mathfrak{d}$.

Demonstração. Se $\mathfrak{b}=\mathfrak{d}$, basta considerar $D=\left\{f_{\alpha}: \alpha<\mathfrak{d}\right\}$ uma família dominante (e não necessariamente bem ordenada), bem como $S=\left\{f \in \omega^{\omega}: f\right.$ é estritamente crescente $\}$ (que também é dominante) e obter $\left\{g_{\alpha}: \alpha<\mathfrak{d}\right\} \subseteq S$ satisfazendo

$$
(\forall \xi<\mathfrak{d})\left(f \in\left\{g_{\alpha}: \alpha<\xi\right\} \cup\left\{f_{\xi}\right\} \Rightarrow f<^{*} g_{\xi}\right)
$$

Obtemos tal família recursivamente. Inicialmente, escolhemos $g_{0} \in S$ tal que $f_{0}<^{*} g_{0}$. Supondo definidos $g_{\alpha}$ para cada $\alpha<\xi$ para algum $\xi<\mathfrak{d}$ satisfazendo a condição acima, segue que a família $G_{\xi}=\left\{g_{\alpha}: \alpha<\xi\right\} \cup\left\{f_{\xi}\right\}$ é necessariamente limitada com respeito a $\leq^{*}$ (pois $|\xi|<\mathfrak{d}=\mathfrak{b}$ ), e assim existe $g \in \omega^{\omega}$ tal que $f \leq^{*} g$ para toda $f \in G_{\xi}$. Escolhemos então $g_{\xi}$ como sendo uma função de $S$ que satisfaz $g(n)<g_{\xi}(n)$ para todo $n \in \omega$. Claramente, $D^{\prime}=\left\{g_{\xi}: \xi<\mathfrak{d}\right\}$ é uma família dominante (do contrário, $D$ não seria dominante) e bem ordenada por $<^{*}$, i.e., $D$ é uma escala ${ }^{3}$.

\footnotetext{
${ }^{2}$ Que já utilizamos implicitamente em certos pontos do texto.

${ }^{3}$ Note que usamos $\mathfrak{b}=\mathfrak{d}$ apenas para garantir a limitação de $\left\{g_{\alpha}: \alpha<\xi\right\}$ (se usássemos $|\xi|<\mathfrak{d}$ para afirmar que $G_{\xi}=\left\{g_{\alpha}: \alpha<\xi\right\} \cup\left\{f_{\xi}\right\}$ não é dominante, obteríamos $g \in \omega^{\omega}$ tal que $g \mathbb{Z}^{*} f$ para toda $f \in G_{\xi}$, o que não seria suficiente para definir $g_{\xi}$ ). Em particular, se em vez da família dominante $D$ utilizássemos uma família ilimitada $F$, o mesmo processo que apresentamos acima nos daria uma família $\left\{g_{\alpha}: \alpha<\mathfrak{b}\right\}$ ilimitada e bem ordenada por $<^{*}$, donde é fácil ver que $\mathfrak{b}=\min \left\{|F|: F\right.$ é ilimitada em $\omega^{\omega}$ e bem ordenada por $\left.<^{*}\right\}$.
} 
Para a recíproca, apresentamos apenas um esboço da demonstração: tome $A$ uma escala em $\omega^{\omega}$ e note que existe uma escala $A^{\prime}=\left\{f_{\alpha}: \alpha<\mathfrak{d}\right\}$ cujo tipo de ordem é $\mathfrak{d}$, donde segue que $\operatorname{cof}(\mathfrak{d})=\mathfrak{d}$ e, por conseguinte, se $E \subset A^{\prime}$ tem cardinalidade estritamente menor do que $\mathfrak{d}$, então $E$ não é uma família ilimitada em $\omega^{\omega}$ (pois, se fosse, seria cofinal em $A^{\prime}$ ), o que nos permite concluir que $\mathfrak{d} \leq \mathfrak{b}(c f$. [42], página 32$)$.

Em particular, note que se $\mathscr{F}=\left\{g_{\alpha}: \alpha<\mathfrak{d}\right\}$ é uma escala em $\omega^{\omega}$, então para cada ordinal limite $\lambda<\mathfrak{d}$ e $n \in \omega$, podemos definir $f_{\lambda+n} \in \omega^{\omega}$ fazendo $f_{\lambda+n}(x)=x \cdot g_{\lambda}(x)+n$. Como todo ordinal $\alpha<\mathfrak{d}$ é da forma $\alpha=\lambda+n$ para algum ordinal limite $\lambda<\mathfrak{d}$ e $n \in \omega$, segue que $\left\{f_{\alpha}: \alpha<\mathfrak{d}\right\}$ é uma escala em $\omega^{\omega}$, bem ordenada por $<^{*}$, mas dominante com respeito a $\leq$, i.e., dada $f \in \omega^{\omega}$ existe $f_{\alpha}$ tal que $f(n) \leq f_{\alpha}(n)$ para todo $n \in \omega$. Vamos chamar uma família $\mathscr{F}$ com tal propriedade de escala forte.

Teorema 4.3.2. Se $\mathfrak{d}=\omega_{1}$, então existe um espaço de Michael

Demonstração. Como $\omega_{1} \leq \mathfrak{b} \leq \mathfrak{d}$, a hipótese do teorema nos dá $\mathfrak{b}=\mathfrak{d}=\omega_{1}$, donde segue pelo lema anterior que existe uma escala $\mathscr{F}=\left\{f_{\alpha}: \alpha<\mathfrak{d}\right\}$, que sem perda de generalidade supomos ser uma escala forte. Seja $X=Y=\omega^{\omega}$ e considere $\rho$ como sendo a $\mathscr{F}$-topologia; em virtude do Teorema 4.2.1, é suficiente mostrarmos que:

(a) $\rho$ contém a topologia usual de $\omega^{\omega}$ (o que vale pela definição de $\rho$ );

(b) $\left(\omega^{\omega}, \rho\right)$ não é de Lindelöf;

(c) se $K \subset \omega^{\omega}$ é $u$-compacto, então $K$ é $\mathscr{F}$-Lindelöf.

O item (b) segue em virtude de $\mathscr{F}$ ser dominante com respeito a $\leq$ : dada $f \in \omega^{\omega}$ existe $f_{\alpha} \in \mathscr{F}$ tal que $f \leq f_{\alpha}$, e assim $f \in K_{f_{\alpha}}$, donde segue que $\left\{K_{f}: f \in \mathscr{F}\right\}$ é uma cobertura por $\rho$-abertos de $\omega^{\omega}$ que não admite subcobertura enumerável (pois não existe família dominante enumerável). Para verificar (c), mostraremos que todo $K \subset \omega^{\omega} u$-compacto tem $\mathscr{F}$-peso enumerável; note que é suficiente mostrar isso para conjuntos da forma $K_{f}$, para $f \in \mathscr{F}$. Como $\mathfrak{d}=\omega_{1}$ segue que se $\alpha<\mathfrak{d}$, então $\left\{f_{\beta}: \beta \leq \alpha\right\}$ é enumerável, e assim basta notar que os conjuntos da forma

$$
\prod_{n \in \omega} V_{n} \cap\left(\bigcap_{f \in I} K_{f}\right),
$$


onde $V_{n} \neq \omega$ para finitos $n \in \omega$ e $I \in\left[\left\{f_{\beta}: \beta \leq \alpha\right\}\right]^{<\omega}$, constituem uma $\mathscr{F}$-base enumerável $\operatorname{para} K_{f_{\alpha}}$.

Observação 4.3.3. Em particular, se $\mathscr{F} \subseteq \omega^{\omega}$ é tal que a $\mathscr{F}$-topologia satisfaz as condições 4.2.1), 4.2.2 e, além disso, todo $u$-compacto de $\omega^{\omega}$ tem $\mathscr{F}$-peso enumerável, então $\mathfrak{d}=\omega_{1}$ (cf. Proposição 3.3 [1] ).

Note que a hipótese $\mathfrak{d}=\omega_{1}$ acarreta tanto $\mathfrak{b}=\omega_{1}$ quanto $\mathfrak{b}=\mathfrak{d}=\operatorname{cov}(\mathcal{M})$. Veremos que cada uma delas é suficiente para garantir a existência de um espaço de Michael.

Para $\mathfrak{b}=\omega_{1}$, basta aplicar o mesmo processo da demonstração do Lema 4.3.1 para uma família ilimitada $B$ e obter uma coleção $Y=\left\{f_{\alpha}: \alpha<\omega_{1}\right\}$ ilimitada (logo, não-enumerável) e bem ordenada por $<^{*}$; observe que se $K \subset \omega^{\omega}$ é compacto na topologia usual, então $K \cap Y$ é necessariamente enumerável (essencialmente por termos $\mathfrak{b}=\omega_{1}$ e $Y$ bem ordenada), donde a existência do espaço de Michael segue pelo Corolário 4.2.5.

A fim de obtermos um espaço de Michael a partir da hipótese $\mathfrak{b}=\mathfrak{d}=\operatorname{cov}(\mathcal{M})$, precisamos do seguinte lema, enunciado em [1]:

Lema 4.3.4. Se $X$ é um espaço métrico compacto e $\mathcal{C}$ é uma cobertura por fechados para $X$ tal que $|\mathcal{C}|<\operatorname{cov}(\mathcal{M})$, então $\mathcal{C}$ admite subcobertura enumerável.

Teorema 4.3.5. $\mathrm{Se} \mathfrak{b}=\mathfrak{d}=\operatorname{cov}(\mathcal{M})$, então existe um espaço de Michael.

Demonstração. Como $\mathfrak{b}=\mathfrak{d}$, podemos tomar $\mathscr{F}=\left\{f_{\alpha}: \alpha<\mathfrak{d}\right\}$ uma escala forte e novamente fazer $X=Y=\omega^{\omega}$ e $\rho$ como sendo a $\mathscr{F}$-topologia nos termos do Teorema 4.2.1. Como no Teorema 4.3.2, o espaço $\left(\omega^{\omega}, \rho\right)$ não é de Lindelöf, e assim basta mostrarmos que se $K \subset \omega^{\omega}$ é $u$-compacto, então $K$ é de $\mathscr{F}$-Lindelöf. Supondo que não seja este o caso, podemos considerar

$$
\beta=\min \left\{\alpha<\mathfrak{d}: \exists K \subseteq K_{f_{\alpha}} \wedge K \text { é } u \text {-compacto e não é } \mathscr{F} \text {-Lindelöf }\right\}
$$

Seja $K \subseteq K_{f_{\beta}}$ o $u$-compacto que não é $\mathscr{F}$-Lindelöf. É fácil ver que $K=\bigcup_{\alpha \leq \beta} K_{f_{\alpha}} \cap K$ e que, pela minimalidade de $\beta$, cada $K_{f_{\alpha}} \cap K$ é $u$-compacto e $\mathscr{F}$-Lindelöf. Seja então $\mathcal{U}$ uma cobertura por $\mathscr{F}$-abertos para $K$ - cada $\mathscr{F}$-aberto de $\mathcal{U}$ é um $\mathscr{F}$-aberto em $\omega^{\omega}$ interceptado com $K$. Vamos mostrar que $\mathcal{U}$ tem subcobertura enumerável. 
Para isso, note primeiramente que para cada $f \in K^{\prime}=K \backslash \bigcup_{\alpha<\beta} K_{f_{\alpha}}$ podemos tomar $U_{f}$ um $u$-aberto de $\omega^{\omega}$ que contém $f$ satisfazendo $U_{f} \cap K \subseteq U$ para algum $U \in \mathcal{U}$. De fato, dado $U \in \mathcal{U}$ tal que $f \in U$, existem $V=\prod_{i \in \omega} V_{i}$ um $u$-aberto básico de $\omega^{\omega}$ e $f_{\alpha_{0}}, \ldots, f_{\alpha_{n}} \in \mathscr{F}$ tais que $f \in V \cap\left(\bigcap_{i \leq n} K_{f_{\alpha_{i}}}\right) \cap K \subseteq U$. Agora, como $f \notin \bigcup_{\alpha<\beta} K_{f_{\alpha}}$, temos necessariamente $\alpha_{j} \not \leq \beta$ para cada $j \leq n$ e, por $\mathscr{F}$ ser uma escala, isso nos permite inferir que $f_{\beta}<^{*} f_{\alpha_{j}}$ ou $f_{\beta}=f_{\alpha_{j}}$. Logo, basta tomar $U_{f}=\prod_{i \in \omega} U_{i}$, onde $U_{i}=V_{i}$ se $f_{\beta}(i) \leq f_{\alpha_{j}}(i)$ para todo $j \leq n$, e

$$
U_{i}=V_{i} \cap\left\{m \in \omega: 0 \leq m \leq \min \left\{f_{\alpha_{j}}(i): f_{\beta}(i)>f_{\alpha_{j}}(i)\right\}\right\}
$$

se $f_{\beta}(i)>f_{\alpha_{j}}(i)$ para algum $j \leq n$ (se $K^{\prime}=\emptyset$, então $K=\bigcup_{\alpha<\beta} K_{f_{\alpha}} \cap K$ e, como cada $K_{f_{\alpha}} \cap K$ é fechado, segue do Lema anterior que $K$ é reunião enumerável de conjuntos $\mathscr{F}$ Lindelöf). Temos assim uma cobertura $\left\{U_{f}: f \in K^{\prime}\right\}$ por $u$-abertos para $K^{\prime}$. Como $\omega^{\omega}$ é hereditariamente Lindelöf, segue que existe $\left\{f_{n}: n \in \omega\right\} \subseteq K^{\prime}$ tal que $K^{\prime} \subseteq \bigcup_{n \in \omega} U_{f_{n}}$. Note então que $K^{\prime \prime}=K \backslash \bigcup_{n \in \omega} U_{f_{n}}$ é $u$-compacto, $\left\{K_{f_{\alpha}} \cap K: \alpha<\beta\right\}$ é uma cobertura por fechados para $K^{\prime \prime}$ e, $\operatorname{como} \beta<\operatorname{cov}(\mathcal{M})$, em virtude do Lema anterior podemos afirmar que $K^{\prime \prime}$ é reunião enumerável de conjuntos $\mathscr{F}$-Lindelöf. Logo, $K^{\prime \prime}$ é $\mathscr{F}$-Lindelöf, donde o resultado segue facilmente. 



\section{Referências Bibliográficas}

[1] Alas, O. T.; Aurichi, L. F.; Junqueira, L. R.; TAll, F. D. Non-productively Lindelöf spaces and small cardinals. Houston J. Math 37 (2011): 1373-1381.

[2] Alster, K. On the class of all spaces of weight not greater than $\omega_{1}$ whose cartesian product with every Lindelöf space is Lindelöf. Fund. Math. 129 (1988), 133-140.

[3] ARHANGEL' SKII, A. V. The frequency spectrum of a topological space and the product operation. Trans. Moscow Math. Soc. 1981, 2: 163-200.

[4] Arhangel's KiI, A. V. Topological function spaces. Kluwer Academic Publishers, Dordrecht, 1992.

[5] Aurichi, L. F.; Bella, A. Productively countably tight spaces and topological games. arXiv:1307.7928, 2013.

[6] BeER, G.; LEVI, S. Strong uniform continuity. Journal of Mathematical Analysis and Applications, 350 (2009): 568-589.

[7] Blass, A. Combinatorial Characteristics of the Continuum, em: Handbook of Set Theory, Vol. 1 (M. Foreman e A. Kanamori, eds.) Springer: 2010.

[8] Bouleau, N. Une structure uniforme sur un espace $\mathcal{F}(E, F)$. Cahiers de Topologie et Géométrie différentielle, Vol XI, 2 (1969): 207-214. 
[9] Caserta, A.; Di Maio, G.; Holá, L. Arzelà's Theorem and strong uniform convergence on bornologies. Journal of Mathematical Analysis and Applications, 371 (2010): 384-392.

[10] Caserta, A.; Di Maio, G.; Kočinac, Lj. D. R. Bornologies, selection principles and function spaces. Topology and Its Applications, 159 (2012): 1847-1852.

[11] Dias, R. R. Jogos Topológicos Seletivos. Notas de aula, Minicurso Verão ICMC, 2013.

[12] Duanmu, H.; Tall, F. D.; Zdomsky, L. Productively Lindelöf and Indestructibly Lindelöf Spaces. arXiv:1210.8010 (2012).

[13] VAn Douwen, E. K. The Integers and Topology, em: Handbook of Set-Theoretic Topology (K. Kunen e Jerry E. Vaughan, eds.). North Holland, Amsterdam, (1984): 111-168.

[14] Di Maio, G.; Kočinac, Lu. D. R.; Meccariello, E. Applications of $k$-covers. Acta Mathematica Sinica, English Series, Vol 22, No. 4 (2006): 1151-1160.

[15] Engelking, R. General Topology: Revised and completed edition. Helderman, Berlin, 1989.

[16] Fremlim, D. H.; Miller, A. W. On some properties of Hurewicz, Menger and Rothberger. Fund. Math. (1988): 17-33.

[17] Folland, G. Real analysis: modern techniques and their applications. 2.ed. John Wiley \& Sons, New York, 1999.

[18] Gerlits, N.; Nagy, Z. Some properties of $C(X)$, I. Topology Appl. 14 (1982): 151161.

[19] Halbeisen, L. Combinatorial Set Theory, With a Gentle Introduction to Forcing. Springer, London, 2012.

[20] Henriksen, M.; Isbell, J. R. Some properties of compactifications. Duke Math. J., 25 (1987) 83-105. 105, 1957. 
[21] Hodel, R. Cardinal Functions I, em: Handbook of Set-Theoretic Topology (K. Kunen e Jerry E. Vaughan, eds.). North Holland, Amsterdam, (1984): 1-62.

[22] Hogbe-Nlend, H. Bornologies and Functional Analysis. North Holland, Amsterdam, 1977.

[23] HrbaceK, K.; Jech, T. Introduction to Set Theory. 3.ed. Marcel Dekker, New York, 1999.

[24] HurewicZ, W. Über eine Verallgemeinerung des Borelschen Theorems. Math. Z. 24 (1925): 401-421.

[25] JECH, T. Set Theory. 3.ed. Springer, Berlin, 2002.

[26] Just, W.; Miller, A. W.; Scheepers, M.; Szeptycki, P. J. The combinatorics of open covers II. Topology Appl. 73 (1996): 241-266.

[27] KoČInAC, LJ. D. R. Closure properties of function spaces. Applied General Topology, Vol. 4, No. 2 (2003): 255-261.

[28] Kомја́тh, P.; Tотік, V. Problems and Theorems in Classical Set Theory. Springer, New York, 2006.

[29] Kunen, K. Set Theory, An Introduction to Independence Proofs. North Holland, Amsterdam, 1980.

[30] Koppelberg, S. General theory of Boolean Algebras, em: Handbook of Boolean Algebras, Vol. 1 (J. Donald Monk e R. Bonnet, eds.). North Holland, Amsterdam, (1989).

[31] McCoy, R. A.; Ntantu, I. Topological Properties of Spaces of Continuous Functions, Lectures Notes in Mathematics, 1315. Springer, Berlin, 1988.

[32] Michael, E. A. The product of a normal space and a metric space need not to be normal. Bull. Amer. Math. Soc. 69 (1963): 375-376.

[33] VAn Mill, J. An introduction to $\beta \omega$, em: Handbook of Set-Theoretic Topology (K. Kunen e Jerry E. Vaughan, eds.). North Holland, Amsterdam, (1984): 503-567. 
[34] Moore, J. T. Some of the combinatorics related to Michael's Problem. Proceedings of the American Mathematical Society, 127 (1999): 2459-2467.

[35] Okuyama, A.; Terada, T. Function spaces, em: Topics in General Topology (K. Morita e J. Nagata, eds.). North Holland, Amsterdam, (1989): 411-458.

[36] PAWLiKowsKi, J. Undetermined sets of point-open games. Fund. Math. 144 (1994): $279-285$.

[37] Rudin, W. Homogeneity problems in the theory of Čech compactifications. Duke Math. J. 23 (1956): 409-419.

[38] SAKaI, M. Property $C^{\prime \prime}$ and function spaces. Proceedings of the American Mathematical Society, Vol. 104, No. 3 (1988): 917-919.

[39] Scheepers, M.Combinatorics of open covers (III): games, $\mathbf{C p}(\mathbf{X})$. Fundamenta Mathematicae 152 (1997): 231-254.

[40] ScheEPERS, M.Remarks on countable tightness. Topology and Its Applications, 161 (2014): 407-432.

[41] Scheepers, M.The length of some diagonalization games. Arch. Math. Logic, 38 (1999): 103-122.

[42] DA SILVA, S. G. Uma Introdução aos Pequenos Cardinais e às suas Aplicações em Topologia. Dissertação de mestrado, Universidade de São Paulo, 1998.

[43] Stone, M. H.The Generalized Weierstrass Approximation Theorem. Mathematics Magazine, Vol. 21, No. 5 (1948): 237-254.

[44] TelgáRsky, R.On games of Topsoe. Math. Scand. 54 (1984): 170-176.

[45] TElgÁRSKy, R.Spaces defined by topological games, II. Fund. Math. 116 (1983): 189207.

[46] TElgÁRSKy, R.Topological games: on the 50th anniversary of the Banach-Mazur game. Rocky Mountain Jounal of Mathematics, Vol. 17, No. 2 (1987): 227-276. 
[47] Tkachuk, V. V. A $C_{p}$-Theory Problem Book, Springer, New York, 2010.

[48] USPENSKII, V.V. Frequency spectrum of functional spaces. Vestnik. Mosk. Universita. Matematika, Vol. 37, No. 1 (1982): 31-35.

[49] Walker, R.C. The Stone-Čech Compactification. Springer, New York, 1974.

[50] Willard, S. General Topology. Addison Wesley, Reading, 1970.

[51] Zdomsky, L. A semifilter approach to selection principles. Comment. Math. Univ. Carolinae 46 (2005): 525-539. 


\section{Índice Remissivo}

$\mathcal{B}$-network, 62

álgebra Booleana, 121

base

compacta, 43

fechada, 43

local, para subconjunto, 58

para a bornologia, 42

para a uniformidade, 28

boa ordem, 18

boa ordenação, 18

bornologia, 42

base compacta para a, 43

base fechada para a, 43

base para a, 42

produto, 43

soma, 44

sub-base para a, 43

trivial, 42

cardinalidade, 19

cobertura

$K$-cobertura, 44
$K_{\delta}$-cobertura, 130

$\gamma_{\mathcal{B}}$-cobertura, 75

$\mathcal{B}_{\delta}$-cobertura, 130

$\omega$-cobertura, 44

$\mathcal{B}$-cobertura, 44

cofinalidade, 19

compactificação, 107

de Stone-Čech, 108

de Alexandroff, 114

conjunto

$G_{\delta}, 90$

$G_{\kappa}, 90$

bornológico, 42

conjuntos equipotentes, 19

dirigido, 21

finito, 19

infinito enumerável, 19

magro, 140

não-enumerável, 19

nunca denso, 140

transitivo, 18

continuidade uniforme forte, 95 
entourage, 28

simétrica, 32

escala, 142

forte, 143

espaço

$\gamma_{\mathcal{B}}$-espaço, 75

$\gamma_{\mathcal{B}}$-espaço estrito, 75

$\kappa$-singular, 89

$\sigma$-compacto, 23

$\mathcal{B}$-Lindelöf, 45

$\aleph_{0}$-espaço, 64

q-espaço, 58

submetrizável,71

Booleano, 123

ccc, 68

compacto, 23

de Alster, 130

de Baire, 140

de Banach, 112

de Fréchet, 72

de Fréchet estrito, 72

de Hausdorff, 23

de Lindelöf, 23

de Lindelöf no infinito, 134

de Menger, 39

de Michael, 141

de Rothberger, 39

de Stone de uma álgebra Booleana, 123

de tipo contável, 137

de Tychonoff, 23 fortemente de Alster, 130

hemicompacto, 58

homogêneo, 53

localmente compacto, 114

normal, 23

ponto-contável, 58

produtivamente $\kappa$-tight, 89

produtivamente de Lindelöf, 129

regular, 23

resíduo do, 108

separável, 25

soma de espaços, 22

$\mathrm{T}_{0}, 22$

$\mathrm{T}_{1}, 23$

$\mathrm{T}_{3}, 23$

$\mathrm{T}_{4}, 23$

$\mathrm{T}_{3 \frac{1}{2}}, 23$

uniforme, 28

zero-dimensional, 123

estratégia

para o jogador I, 36

para o jogador II, 37

vencedora para o jogador I, 37

vencedora para o jogador II, 37

família

dominante, 139

ilimitada, 139

família $\kappa$-singular, 89

fecho, 21

filtro, 122 
função

cardinal, 25

cofinal, 19

diagonal, 108

quociente, 99

semicontínua superiormente, 135

valorada em compactos, 135

função cardinal

$\mathcal{B}$-net-peso, 62

$\mathcal{B}$-peso, 69

caráter, 55

celularidade, 68

densidade, 56

grau de $\mathcal{B}$-Arens, 56

grau de $\mathcal{B}$-Lindelöf, 45

grau de Alster, 138

grau de Lindelöf, 45

net-peso, 61

peso, 56

peso fraco, 64

tightness, 72

Hipótese do Contínuo, 19

interior, 21

jogo

de comprimento $\alpha, 34$

de Menger, 39

de Rothberger, 39

indeterminado, 37

número cardinal, 19

de cobertura, 140

de dominância, 139

de ilimitância, 139

natural, 19

ordinal, 18

net, 21

convergente, 21

uniformemente convergente, 30

network, 50

ordinal

aditivamente indecomponível, 81

limite, 19

sucessor, 19

P-ponto, 121

partida, 37

extensão de partidas iniciais, 87

início de, 87

primeiro axioma de enumerabilidade, 25

Problema de Michael, 141

propriedade de caráter finito, 68

reta de Sorgenfrey, 129

segundo axioma de enumerabilidade, 25

sequência, 19

sub-base

para a bornologia, 43

sucessor, 19

suporte, 22 
tipo de ordem, 19

topologia

$\kappa$-modificação da, 90

$\mathscr{F}$-topologia, 142

compacto-aberta, 32

da $V$-convergência, 96

da convergência pontual, 25

da convergência uniforme, 26

da convergência uniforme em $\mathcal{B}, 50$

da convergência uniforme em compactos,

33

da convergência uniforme forte em $\mathcal{B}, 95$

da ordem, 22

fraca, 109

produto, 22

ultrafiltro, 122

livre, 125

uniformidade, 28

metrizável, 31

pseudo-metrizável, 31

vizinhança, 21 